\title{
Desanclaje térmico de fluxones en anillos de uniones Josephson
}

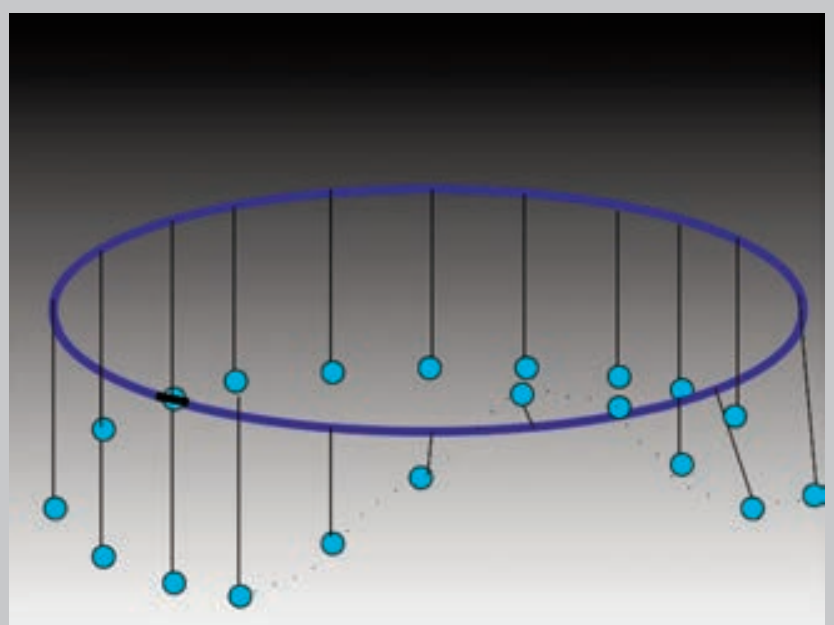

Fernando Naranjo Mayorga 

Desanclaje térmico de Fluxones en Anillos de Uniones Josephson 


\section{Colección de Estudios de Física}

\section{Vol. 85}

Esta colección recoge las tesis presentadas en el Departamento de Física de la Materia Condensada de la Universidad de Zaragoza desde su constitución en $198 \%$. 


\section{Desanclaje Térmico de Fluxones en Anillos de Uniones Josephson}

Fernando Naranjo Mayorga

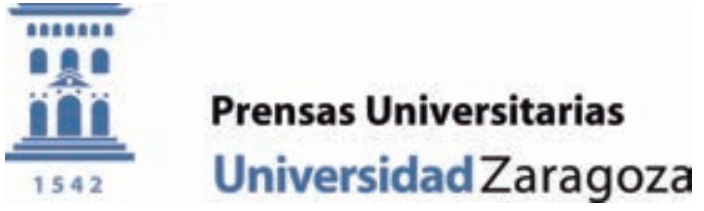




\section{NARANJO MAYORGA, Fernando}

Desanclaje térmico de fluxones en anillos de uniones Josephson / Fernando Naranjo Mayorga. - Zaragoza : Prensas Universitarias de Zaragoza, 2011

XIV, 163 p. ; 25 cm. - (Colección de estudios de física ; 85)

Bibliografía: p. 155-163. - ISBN 978-84-15274-63-6

Superconductores-Tesis doctorales

537.3(043.2)

$621.315 .5(043.2)$

Cualquier forma de reproducción, distribución, comunicación pública o transformación de esta obra solo puede ser realizada con la autorización de sus titulares, salvo excepción prevista por la ley. Diríjase a CEDRO (Centro Español de Derechos Reprográficos, www.cedro.org) si necesita fotocopiar o escanear algún fragmento de esta obra.

(C) Fernando Naranjo Mayorga

(C) De la presente edición, Prensas Universitarias de Zaragoza

1. ${ }^{\mathrm{a}}$ edición, 2011

Prensas Universitarias de Zaragoza. Edificio de Ciencias Geológicas, c/ Pedro Cerbuna, 12, 50009 Zaragoza, España. Tel.: 976761 330. Fax: 976761063

puz@unizar.es http://puz.unizar.es

Impreso en España

Imprime: Servicio de Publicaciones. Universidad de Zaragoza

D.L.: Z-4135-2011 
a mi silencio 



\section{Índice}

Prólogo XIII

1. Introducción 1

1.1. Unión Josephson . . . . . . . . . . . . . . . . . . . . . . . . 1

1.1.1. Superconductividad ... . . . . . . . . . . 1

1.1.2. Efecto Josephson . . . . . . . . . . . . . . . . 2

1.1.3. Uniones túnel superconductoras. Ecuaciones de Josephson 4

1.1.4. Modelo RCSJ de una unión . . . . . . . . . . . . . 6

1.1.5. JJ y ciencia no lineal . . . . . . . . . . . . . . . . 6

1.1.6. Curva IV . . . . . . . . . . . . . . . . . . . . 7

1.1.7. Uniones Josephson "largas" . . . . . . . . . . . . . . . 9

1.2. Efecto de la temperatura . . . . . . . . . . . . . . . . 10

1.2.1. Adición de ruido térmico . . . . . . . . . . . . 10

1.2.2. Modelo de salto de barrera y efecto de la temperatura . 10

1.2.3. El "problema de Kramers" y uniones Josephson . . . . . 12

1.3. Redes de uniones Josephson . . . . . . . . . . . . . . . . . . 13

1.3.1. Redes en serie . . . . . . . . . . . . . . . . . . . 14

1.3.2. rf-SQUID . . . . . . . . . . . . . . 15

1.3.3. dc-SQUID . . . . . . . . . . . . . . . . . . 16

1.3.4. Red de uniones Josephson en paralelo . . . . . . . . . 17

1.3.5. La escalera de uniones Josephson . . . . . . . . . . . . 18

1.3.6. Redes bidimensionales . . . . . . . . . . . . . . 19

2. El anillo de uniones Josephson $\quad 21$

2.1. Ecuaciones de la dinámica del sistema . . . . . . . . . . . . 21

2.2. Energía del sistema . . . . . . . . . . . . . . 25

2.2.1. Configuraciones de mínima energía . . . . . . . . . 27

2.3. Anillo de uniones Josephson y física no lineal . . . . . . . . 28

2.3.1. Modelo Frenkel-Kontorova (FK) . . . . . . . . . 28

2.3.2. La ecuación de sine-Gordon . . . . . . . . . . . . . 30

2.3.3. Solitones en física no lineal . . . . . . . . . . . . . . 31

2.3.4. Solitones en el anillo de uniones Josephson . . . . . . . . 32 
2.4. Efecto Ratchet en ciencia no-lineal . . . . . . . . . . . . . . 34

2.4.1. Rocking ratchet . . . . . . . . . . . . . 36

2.4.2. Flashing ratchet . . . . . . . . . . . . . . 37

2.5. Dispositivos ratchet para fluxones . . . . . . . . . . . 38

3. Fluxón como una partícula $\quad 43$

3.1. Redes regulares . . . . . . . . . . . . . . . . . . . 43

3.1.1. La barrera y el potencial de Peierls-Nabarro . . . . . . 45

3.1.2. Espectro de modos lineales . . . . . . . . . . . . . 48

3.1.3. La masa efectiva del fluxón . . . . . . . . . . . . . . 51

3.1.4. Un experimento numérico . . . . . . . . . . . . 53

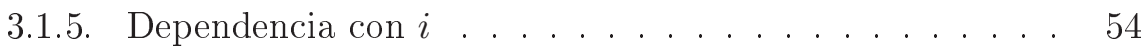

3.2. Redes Ratchet. . . . . . . . . . . . . . . . . . . . 55

3.2.1. Red ratchet de periodo $2 \ldots \ldots \ldots \ldots$

3.2.2. Red ratchet de periodo $3 \ldots \ldots \ldots$. . . . . . 58

4. Desanclaje Térmico de Fluxones en Redes regulares $\quad 67$

4.1. Introducción . . . . . . . . . . . . . . . . 67

4.2. Red regular con 9 uniones y un fluxón . . . . . . . . . . . . 71

4.2.1. Consideraciones sobre la rampa . . . . . . . . . . 71

4.2.2. Curvas $I-V$ a temperatura nula: Regímenes de Amortiguamiento . . . . . . . . . . . . . 73

4.2.3. Curvas $I$ - $V$ : el efecto de la temperatura . . . . . . . . 76

4.2.4. Desanclaje térmico del fluxón: $\left\langle i_{\mathrm{dep}}(T)\right\rangle \ldots$. . . . . . 78

4.2.5. Comparación del escape del fluxón con el escape térmico de partícula en un potencial metaestable . . . . . . . . 80

4.2.6. Difusión del Fluxón . . . . . . . . . . . . . . . . . 86

4.2.7. Tasas de escape . . . . . . . . . . . . . . . . . . . . . . . . . 91

4.3. Red regular de 30, 60, 120 y 300 uniones . . . . . . . . . . . 93

4.3.1. Curvas $I-V \ldots \ldots \ldots \ldots \ldots \ldots$

4.3.2. Estudio de $\left\langle i_{\mathrm{sw}}(T)\right\rangle$ para sistemas de diferentes tamaños 98

4.4. Red regular de 9 uniones con 2 y 3 fluxones . . . . . . . . . . . 101

4.4.1. 2 fluxones . . . . . . . . . . . . . . . . . 102

4.4.2. 3 fluxones . . . . . . . . . . . . . . . . . 104

4.4.3. Comparación entre los sistemas de 9 uniones Josephson con diferente número de fluxones . . . . . . . . . . 105

4.4.4. Resultados sobre $\left\langle i_{\mathrm{dep}}(T)\right\rangle,\left\langle i_{\mathrm{sw}}(T)\right\rangle$, у $\sigma \ldots \ldots . . . .106$

5. Desanclaje térmico de fluxones en redes ratchet 109

5.1. Introducción . . . . . . . . . . . . . . . . . . . . . . . 109

5.2. Ecuaciones . . . . . . . . . . . . . . . . . . 110

5.3. Red ratchet con 9 uniones y un fluxón . . . . . . . . . . . . 111 


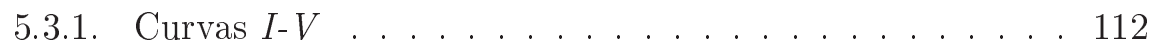

5.3.2. $\left\langle i_{\mathrm{dep}}(T)\right\rangle \ldots \ldots \ldots \ldots \ldots \ldots \ldots \ldots$

5.4. Red ratchet con 9 uniones y dos fluxones . . . . . . . . . . 122

5.4.1. Curvas $I-V \ldots \ldots \ldots \ldots \ldots \ldots \ldots$

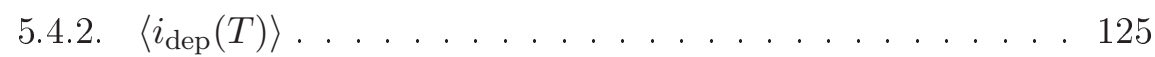

6. Activación térmica en el régimen de amortiguamiento débil. 129

6.1. Teoría de Kramers estándar . . . . . . . . . . . . . . . . 130

6.1.1. Teoría de Kramers para amortiguamiento débil . . . . . 131

6.1.2. Extensiones de la teoría de Kramers al régimen de amor-

tiguamiento moderado-débil. . . . . . . . . . . . 133

6.2. Correcciones de barrera finita . . . . . . . . . . . . . 135

6.3. Tasa de escape: resultados numéricos . . . . . . . . . . . . . . 139

6.4. El problema de las condiciones iniciales . . . . . . . . . . . . . 143

6.5. Resultados sobre la corriente de switching . . . . . . . . . . . 145

6.6. Discusión . . . . . . . . . . . . . . . . . 147

$\begin{array}{lr}\text { Discusión y conclusiones } & 149\end{array}$

$\begin{array}{ll}\text { Bibliografía } & 155\end{array}$ 



\section{Prólogo}

El trabajo realizado en esta tesis doctoral está centrado en el estudio computacional del efecto de la temperatura en las propiedades dinámicas, fundamentalmente en el desanclaje o "depinning" de fluxones en una serie de dispositivos superconductores basados en uniones Josephson. Desde este punto de vista, podría decirse que se trata de un trabajo de física de estado sólido, o de un trabajo en el campo de la superconductividad aplicada o incluso propio del mundo de la ingeniería de dispositivos superconductores.

Por otro lado, hemos de tener presente, que una unión Josephson supone una excelente realización experimental de uno de los sistemas-modelo más importantes de la física no lineal: el péndulo. Así, una red de uniones Josephson acopladas entre sí corresponde a una modelización de un conjunto de osciladores no lineales acoplados. Como veremos, el sistema elegido, un anillo formado por uniones conectadas en paralelo, se corresponde con un conjunto de péndulos unidos por muelles de torsión. Entre otras cosas, dicho sistema es muy interesante por poseer un tipo especial de solución: los famosos solitones, uno de los objetos paradigma de la ciencia no lineal. Desde un punto de vista físico un solitón se corresponde con un cuanto de flujo magnético o fluxón en el sis-
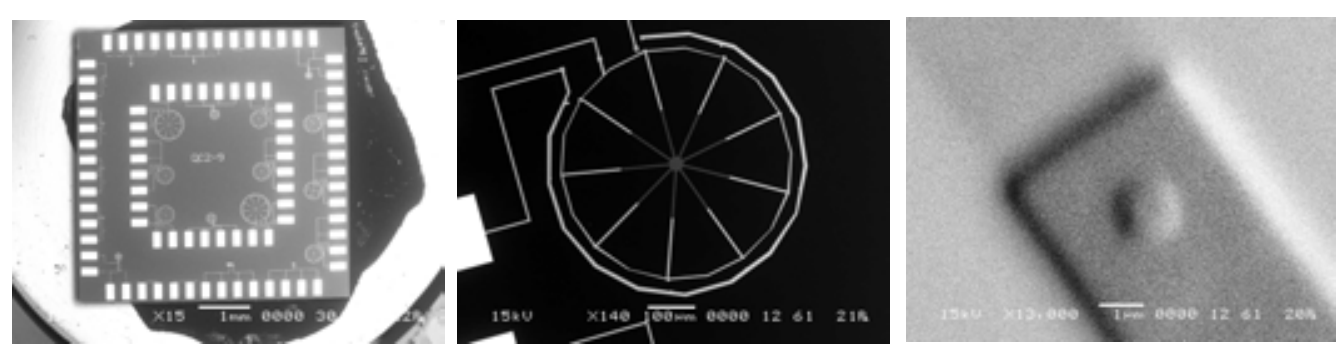

Figura 1: Fotografía de un chip en el que se han diseñado muchos anillos superconductores con uniones Josephson para la realización de distintos experimentos (izquierda). Fotografía de uno de los anillos, formado por nueve uniones (centro). Ampliación mostrando una de las uniones Josephson (derecha). Cortesía del Dr. K. Segall, Colgate University, EEUU. 
tema superconductor y la configuración en anillo es especialmente interesante por posibilitar que una vez creados dichos fluxones o solitones queden atrapados en el sistema. Por tratarse de un sistema discreto, nuestros solitones (los llamaremos kinks en muchas ocasiones, ya que la palabra solitón suele reservarse a estos objetos en el continuo) están anclados a la red y por lo tanto sufren un proceso de desanclaje en presencia de campos externos, proceso que como veremos se ve afectado de manera importante por el efecto de la temperatura. Desde este punto de vista, podría decirse que nuestro trabajo es un trabajo de ciencia básica en el campo de los sistemas dinámicos no lineales, en este caso perturbados por ruido, y las conclusiones obtenidas pueden tener interés en ámbitos muy lejanos al de la física de sistemas superconductores.

Estas memorias son parte de la experiencia de vida, inmerso en una nueva cultura y con el apoyo e interacción de formidables seres humanos y excelentes científicos comenzando por Juanjo y los demás del Non Linear and Statistical Physics Group, y los miembros del Departamento de Física de la Materia Condensada, a todos ellos quiero expresarles mi profunda gratitud. 


\section{Capítulo 1}

\section{Introducción}

Tal y como hemos expuesto en el prólogo de esta memoria, el trabajo realizado puede ser introducido tanto desde la perspectiva de la física de los dispositivos superconductores basados en uniones Josephson como desde la perspectiva de sistemas dinámicos no lineales, dos perspectivas complementarias y a las que nos referiremos numerosas veces en lo que sigue. En cualquier caso, dado que la física es una ciencia eminentemente experimental y que ha sido un conjunto de experimentos concretos la primera motivación de la realización de este trabajo de tesis doctoral, comenzaré la introducción del mismo con este capítulo que resume algunos de los aspectos más sobresalientes de la física de una unión Josephson desde la perspectiva del trabajo realizado y su conexión con la física de los sistemas dinámicos no lineales.

\subsection{Unión Josephson}

\subsubsection{Superconductividad}

La superconductividad es un fenómeno natural que se presenta en circunstancias especiales de temperatura y presión. Muchos materiales no exhiben sus propiedades superconductoras hasta que en ellos la temperatura se aproxima al cero absoluto, por esta razón el descubrimiento de la superconductividad tuvo que esperar hasta el desarrollo tecnológico de la criogenia. Kamerlingh Onnes [1,2] investigó la resistencia eléctrica en metales a baja temperatura enfriando las muestras con helio líquido a $4.2 \mathrm{~K}$ y 1 atmósfera de presión. Después de una serie de experimentos realizados por su asistente, Gilles Holst, quien observó que la resistencia eléctrica del mercurio, caía en picada a cero, cuando se sometía a un baño de helio líquido, Onnes reportó que por debajo de una temperatura crítica, el mercurio pasaba a un nuevo estado, el cual 
teniendo en cuenta sus sorprendentes propiedades eléctricas, se denominó, estado superconductor. Actualmente se encuentran materiales superconductores a temperaturas por encima de los 90 grados Kelvin, lo que facilita y economiza a la vez su observación pues posibilita el enfriamiento con nitrógeno líquido y permite ampliar las aplicaciones de la superconductividad. Además de conductividad perfecta, la superconductividad está caracterizada por el efecto Meissner; esto es, la capacidad del material de expulsar el campo magnético de su interior (diamagnetismo perfecto) $[3,4]$.

La superconductividad es un fenómeno inherentemente mecano cuántico que se manifiesta en si mismo a escala macroscópica. Muchas de sus propiedades pueden ser entendidas en el marco de un modelo cuántico macroscópico (MQM). Esta descripción (MQM), no solo abarca los resultados del modelo clásico, sino también describe autoconsistentemente otras propiedades de los superconductores de relevante aplicación, por ejemplo, un aparato electrónico superconductor conocido como unión Josephson [5, 6], el cual es el alma de la mayoría de dispositivos superconductores de pequeña escala. Estas uniones forman la base de magnetómetros sensibles, capaces de detectar campos magnéticos producidos por el cerebro humano, los cuales son del orden de $10^{-15}$ Tesla. Además, la respuesta de una unión Josephson a la radiación electromagnética forma la base del estándar de voltaje actual [7, 8, 9]. En la actualidad, dispositivos basados en uniones Josephson se usan para investigar problemas fundamentales de la mecánica cuántica y realizar propuestas de procesado cuántico de la información y computación cuántica, ver por ejemplo $[10,11,12,13,14,15]$

\subsubsection{Efecto Josephson}

En su trabajo de 1962 [5] Brian Josephson estudió el túnel de pares de Cooper entre dos metales superconductores y predijo el renombrado efecto Josephson. Desde entonces se han realizado centenares de trabajos en los que se estudia el comportamiento de uniones individuales, de redes de uniones Josephson o de otros dispositivos más complejos que contienen uniones Josephson $[3,4,6,7,8,9]$.

Merece la pena decir no obstante que aunque el efecto Josephson fue introducido y ha sido estudiado fundamentalmente en el contexto de la superconductividad, la física conocida bajo el sobrenombre de efecto Josephson se aplica a otros sistemas cuántico macroscópicos débilmente acoplados [16]. Algunos ejemplos son el efecto Josephson entre superfluidos débilmente acoplados (ver Ref. [17] por ejemplo) y el reciente interés sobre efecto Josephson en condesados de Bose-Einstein débilmente acoplados (ver Ref. [18] por ejemplo). 


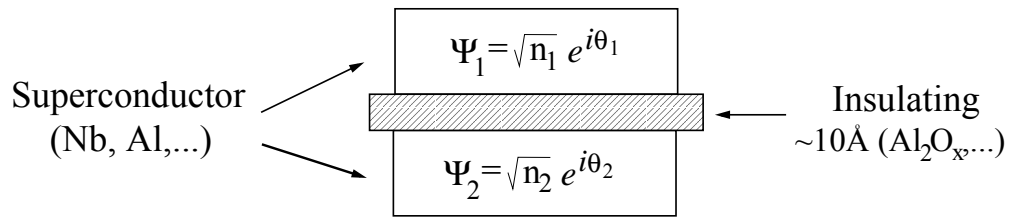

Figura 1.1: Esquema de una unión Josephson de tipo túnel, formada por dos metales superconductores separados por una fina barrera de aislante.

Con respecto al efecto Josephson en superconductores existen diversos tipos de uniones y geometrías que lo presentan. Josephson estudió el caso de una unión tipo túnel. En este caso dos superconductores están separados por una barrera de aislante y el transporte de corriente ocurre mediante el túnel de pares de Cooper entre los dos electrodos superconductores de la unión (unión superconductor-aislante-superconductor o SIS, figura 1.1). Las uniones de tipo túnel son las más habituales y a ellas nos referiremos en nuestro trabajo. Otra opción es una unión formada por dos superconductores separados por un metal en estado normal (unión SNS). Otro dispositivo que presenta efecto Josephson es un micro-puente. El micro-puente está formado por un estrechamiento en un superconductor. Si el estrechamiento es suficientemente pequeño en esa zona se rompe la superconductividad y las dos zonas superconductoras quedan acopladas débilmente a través del micro-puente. Por último nos referiremos a los contactos puntuales. Sea por ejemplo un superconductor que acaba en una punta que a su vez toca a otro superconductor. Las reducidas dimensiones del contacto en la punta hacen que en esa zona se rompa la superconductividad y el acoplo entre los superconductores sea débil.

En el marco del modelo cuántico macroscópico (MQM) de la superconductividad, el estado superconductor se describe por una función de onda mecano cuántica

$$
\psi(\vec{r}, t)=|\psi(\vec{r}, t)| \mathrm{e}^{i \theta(\vec{r}, t)}
$$

donde la densidad local de pares de Cooper está dada por

$$
n_{s}(\vec{r}, t)=|\psi(\vec{r}, t)|^{2}
$$

La fase $\theta(\vec{r}, t)$ juega un papel esencial en la descripción de las propiedades de transporte del superconductor. A partir de la ecuación de Schrödinger para una partícula cargada en un campo electromagnético puede obtenerse la densidad de corriente superconductora

$$
\mathbf{J}_{\mathbf{s}}=q^{*} \operatorname{Re}\left\{\psi^{*}\left(\frac{\hbar}{i m^{*}} \nabla-\frac{q^{*}}{m^{*}} \mathbf{A}\right) \psi\right\},
$$




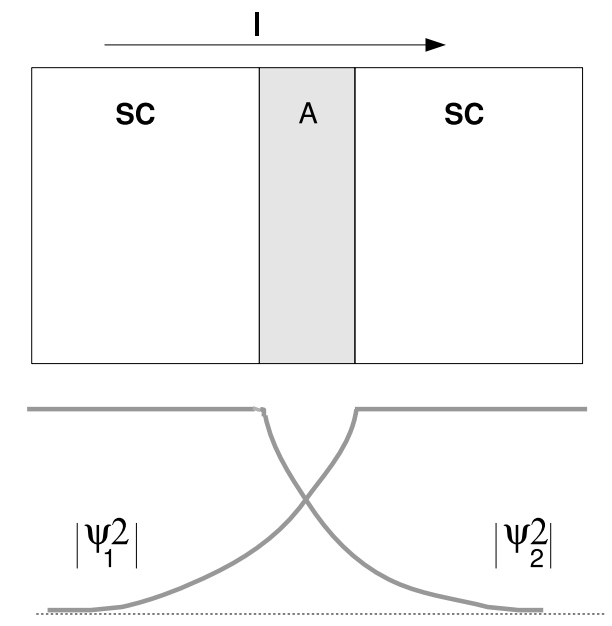

Figura 1.2: El solapamiento de las funciones de onda $\psi_{1}$ y $\psi_{2}$ a ambos lados de la unión es la base del efecto Josephson.

que podemos rescribir como

$$
\mathbf{J}_{\mathbf{s}}=q^{*} n_{s}^{*}(\mathbf{r}, t)\left(\frac{\hbar}{m^{*}} \nabla \theta(\mathbf{r}, t)-\frac{q^{*}}{m^{*}} \mathbf{A}(\mathbf{r}, t)\right)
$$

Puede verse que la corriente superconductora, un observable del sistema, depende de la fase de la función de ondas macroscópica y el potencial vector, que no pueden ser medidos experimentalmente por depender de la elección de gauge. Resulta entonces natural introducir el gradiente de fase invariante gauge $\varphi$ definido por

$$
\varphi=\nabla \theta-\frac{q^{*}}{\hbar} \mathbf{A}=\nabla \theta+\frac{2 \pi}{\Phi_{0}} \mathbf{A}
$$

(la carga del par de Cooper es -2e y el cociente $\hbar / 2 e$ suele escribirse en función del cuanto de flujo magnético $\Phi_{0}=h / 2 e$, así $\left.\hbar / 2 e=\Phi_{0} / 2 \pi\right)$.

El fundamento físico una unión Josephson, se basa en el solapamiento de las funciones de ondas a ambos lados de la unión (figura 1.2).

\subsubsection{Uniones túnel superconductoras. Ecuaciones de Joseph- son}

Una unión Josephson de tipo túnel es un dispositivo de la física del estado sólido formado por dos electrodos superconductores (normalmente Niobio o Aluminio) separados por una barrera fina de aislante (normalmente un óxido de Aluminio), ver Fig. 1.1. 


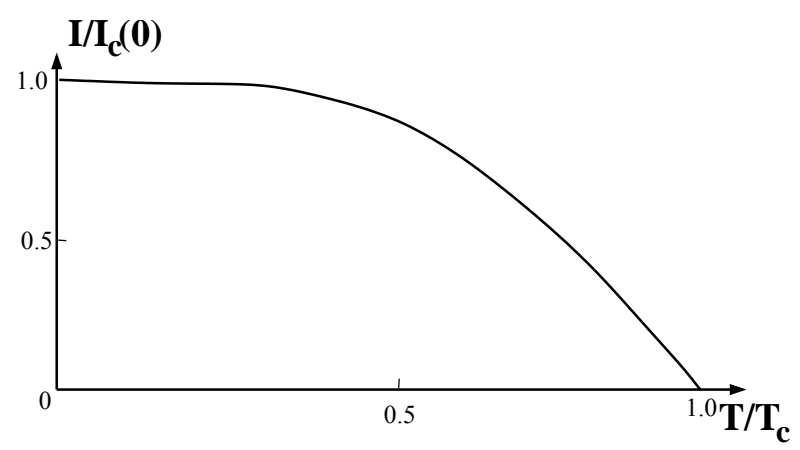

Figura 1.3: Dependencia con la temperatura de la corriente crítica de una unión.

Josephson encontró que el comportamiento de la unión está controlado por el valor de la diferencia de fase invariante gauge entre los electrodos superconductores

$$
\varphi=\theta_{1}-\theta_{2}-\frac{2 e}{\hbar} \int_{1}^{2} \vec{A}(\vec{r}, t) \overrightarrow{d l},
$$

donde $\theta_{i}$ es la fase de la función de ondas macroscópica en el electrodo $i$ ( $\psi_{i}=$ $\left.\sqrt{\left|\psi_{i}\right|} e^{i \theta_{i}}\right)$ y $\vec{A}$ el potencial vector.

Las ecuaciones básicas del efecto Josephson son:

$$
I_{s}=I_{c} \sin \varphi
$$

y

$$
V=\frac{\hbar}{2 e} \frac{d \varphi}{d t}
$$

A partir de ellas podemos ver que, efecto Josephson DC, es posible tener una corriente superconductora a través de la unión con voltaje 0 (entonces $\varphi$ es constante). Esta corriente tiene un valor máximo posible que está dado por $I_{c}$, la corriente crítica de la unión. Sin embargo, efecto Josephson $A C$, si la unión es sometida a un voltaje constante, ésta responde con una corriente alterna de frecuencia dada por $2 \mathrm{eV} / \hbar(483.6 \mathrm{GHz} / \mathrm{mV})$.

La energía potencial asociada con las supercorrientes a través de la unión está dada por

$$
U_{J}=-E_{J} \cos \varphi,
$$

con $E_{J}=\hbar I_{c} / 2 e$. Un primer requisito para observar el efecto Josephson es que la energía Josephson exceda la energía térmica $E_{J} \gg k_{B} T\left(I_{c} \gg 2 e k_{B} T / \hbar\right)$.

La corriente crítica de la unión $I_{c}$ depende de manera importante de la temperatura (Fig. 1.3). Dicha dependencia es normalmente aproximada por la ecuación de Ambegaokar-Baratoff [19]

$$
I_{c} R_{n}=\frac{\pi \Delta}{2 e} \tanh \left(\Delta / 2 k_{B} T\right) .
$$


Aquí $R_{n}$ es la resistencia normal de la unión y $\Delta(T)$ el gap de energía superconductor. A $T=0$ tenemos $I_{c} R_{n}=\pi \Delta(0) / 2 e$ con $\Delta(0)=1.764 k_{B} T_{c}$ y para $T \rightarrow T_{c}, I_{c} R_{n} \simeq\left(2.34 \pi k_{B} / e\right)\left(T_{c}-T\right)$.

\subsubsection{Modelo RCSJ de una unión}

Para estudiar la curva intensidad-voltaje, curva IV o curva característica de la unión, usamos el llamado modelo RCSJ (resistively and capacitively shunted junction) [20, 21] (ver Fig. 1.4). En este modelo la corriente total a través de la unión es la suma de tres contribuciones: la corriente superconductora Josephson (debida al túnel de pares de Cooper), una corriente resistiva normal (debida al túnel de portadores normales) y un canal capacitivo (asociado con la capacidad de la unión). $I=I_{J}+I_{R}+I_{C} \operatorname{con} I_{J}=I_{c} \sin \varphi, I_{R}=V / R$ y $I_{C}=C d V / d t$. Entonces

$$
I=C \dot{V}+\frac{1}{R} V+I_{c} \sin \varphi
$$

Si aplicamos la segunda relación de Josephson $\left[V=\left(\Phi_{0} / 2 \pi\right)(d \varphi / d t)\right]$ y normalizamos la corriente con respecto la corriente crítica de la unión, $i=I / I_{c}$, tiempo respecto a la frecuencia de plasma de la unión $\omega_{p}=\sqrt{2 \pi I_{c} / \Phi_{0} C}$ e introducimos el parámetro de amortiguamiento $\Gamma=\sqrt{\Phi_{0} / 2 \pi I_{c} C R^{2}} 1$, obtenemos

$$
i=\mathcal{N}(\varphi)=\ddot{\varphi}+\Gamma \dot{\varphi}+\sin \varphi
$$

\subsubsection{JJ y ciencia no lineal}

La ecuación 1.12 describe de forma adimensional la dinámica de la diferencia de fase de la unión como función del parámetro de amortiguamiento de la misma y de la corriente normalizada que atraviesa la unión. Esta ecuación es idéntica a la ecuación que describe las oscilaciones (y rotaciones) de un péndulo no lineal forzado y amortiguado en un campo gravitacional y también es idéntica a la ecuación que describe la dinámica de una partícula en un potencial sinusoidal inclinado (the tilted washboard potential): $U(\varphi)=-E_{J} \cos \varphi-(\hbar I / 2 e) \varphi$ [masa $m=(\hbar / 2 e)^{2} C$ y amortiguamiento $\gamma=(\hbar / 2 e)^{2}(1 / R)$ ], ver Fig. 1.4. Ambos sistemas son analogías mecánicas sencillas de la unión e ilustran porqué los dispositivos con uniones Josephson son sistemas experimentales ideales para estudiar aspectos básicos de la física no lineal.

\footnotetext{
${ }^{1} \mathrm{El}$ amortiguamiento en ocasiones es definido en términos del factor de calidad de la unión $Q=1 / \Gamma$ o del parámetro de Stewart-McCumber $\beta_{c}=1 / \sqrt{\Gamma}=2 \pi I_{c} C R^{2} / \Phi_{0}$
} 

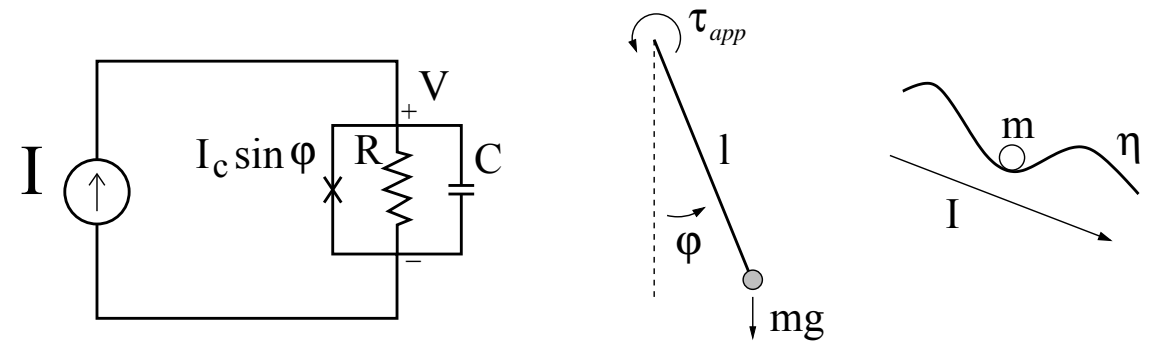

Figura 1.4: Circuito para el modelo RCSJ de la unión y dos analogías mecánicas: el péndulo forzado y amortiguado y la partícula en un potencial sinusoidal inclinado.
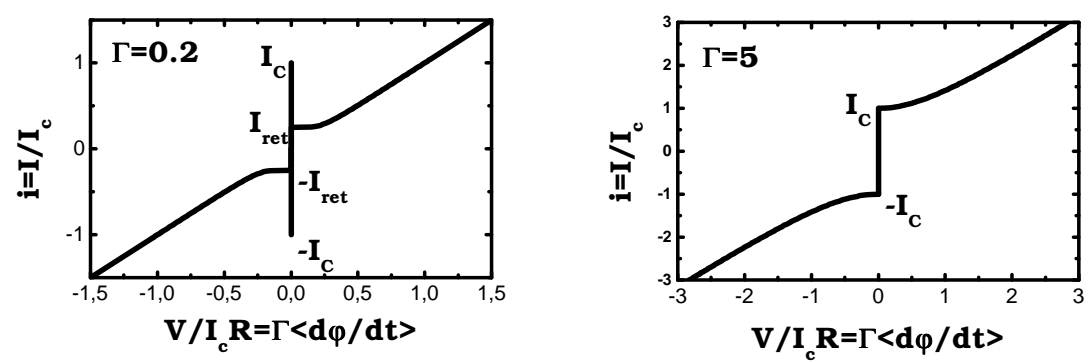

Figura 1.5: Curva IV de una unión Josephson según el modelo RCSJ habitual, $R=$ cte. (Eq. 1.12). Para $\Gamma=0.2$ coexisten dos soluciones distintas en el rango de corrientes entre la corriente crítica, $I_{c}$ y la de reatrapamiento, $I_{\text {rep }}$ (caso de amortiguamiento bajo). Para $\Gamma=5.0$ (caso sobreamortiguado) el voltaje es una función única de la corriente.

\subsubsection{Curva IV}

La figura 1.5 muestra una curva IV calculada numéricamente para una unión a dos valores distintos del amortiguamiento. Para amortiguamientos grandes (figura con $\Gamma=5$ ) el voltaje es una función única de la corriente y aumenta de manera continua desde cero en cuanto $I>I_{c}$ y se aproxima a la relación óhmica ( $I=V / R$, o $i=\Gamma\langle d \varphi / d \tau\rangle$ en unidades normalizadas) a corrientes altas. Para valores menores del amortiguamiento la curva IV presenta histéresis (ver figura con $\Gamma=0.2$ ). Al aumentar el valor de la corriente, en $I=I_{C}$ la unión salta del estado de voltaje cero a la rama resistiva $I=V / R$. Si ahora se decrece la corriente, el voltaje decrece continuamente y se anula para $I=I_{\text {ret }}$ (corriente de reatrapamiento). Para valores suficientemente pequeños del amortiguamiento $I_{\text {ret }} / I_{c} \simeq 4 \Gamma / \pi$.

La figura 1.6 muestra una curva experimental IV en una unión de tipo túnel Niobium-Aluminum Oxide-Niobium. Se observa que a $I_{c}$ el voltaje de la unión salta desde 0 hasta el valor del voltaje de gap $V_{g}=2 \Delta(T) / e\left(V_{g} / I_{c} R_{n}=4 / \pi\right.$ a baja $T$ ). Este voltaje corresponde a la energía necesaria para romper los 


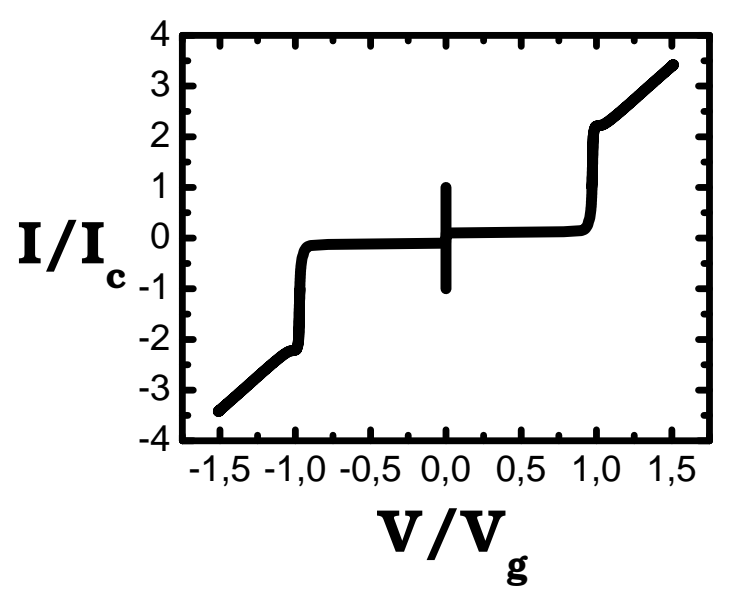

Figura 1.6: Esquema de una curva IV experimental de una unión individual. La resistencia de la unión para valores de voltaje inferiores al voltaje de gap (resistencia subgap) es marcadamente diferente del valor de la resistencia por encima del voltaje de gap (resistencia normal).

pares de Cooper. A valores mayores de la corriente, el voltaje se incrementa y sigue una dependencia óhmica con resistencia dada por la resistencia en estado normal $R_{n}$. Si se decrece la corriente, el voltaje disminuye hasta alcanzarse el voltaje de gap y luego retorna a cero para valores pequeños de la corriente. Esta dependencia no-lineal de la curva muestra la existencia de dos regímenes de disipación muy diferentes en la unión, uno para voltajes por encima del gap y otro para voltajes inferiores al gap. Las propiedades de transporte por encima del gap están gobernadas por los electrones en estado normal, mientras que las propiedades de transporte para voltajes inferiores al gap están usualmente determinadas por la densidad de las cuasi-partículas (electrones individuales en un mar de pares de Cooper).

Una aproximación teórica sencilla para modelar este comportamiento es utilizar el modelo RCSJ con una resistencia no lineal $R(V)$ tal que $R=R_{n}$ si $V>V_{g}$ y $R=R_{s g}(T)$ si $V<V_{g}$. En las uniones tipo túnel la resistencia subgap normalmente exhibe una dependencia muy importante con la temperatura dada por $R_{s g}(T) \simeq R_{n} e^{\Delta / k_{B} T}$.

Una expresión del túnel de cuasi-partículas válida para $k_{B} T \ll \Delta$ y $V<V_{g}$ está dada por [9]

$$
I_{q p}=\frac{2}{e R_{n}} e^{-\Delta / k_{B} T}\left(\frac{2 \Delta}{e V+2 \Delta}\right)^{1 / 2}(e V+\Delta) \sinh \left(\frac{e V}{2 k_{B} T}\right) K_{0}\left(\frac{e V}{2 k_{B} T}\right) .
$$



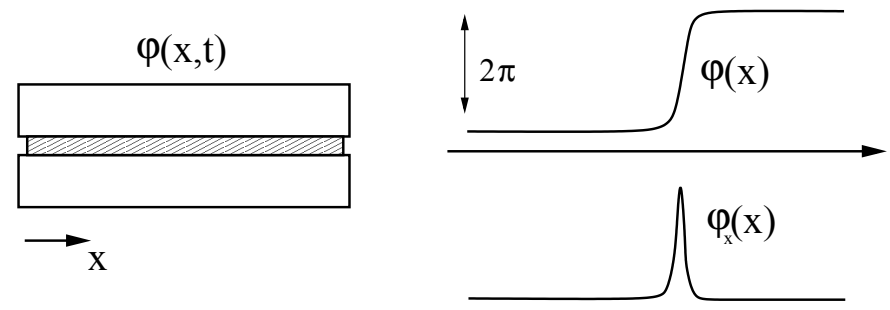

Figura 1.7: Esquema de una unión Josephson larga (izquierda) y dependencia espacial de la fase y su derivada para una unión con un solitón (derecha).

Por otro lado, en algunas investigaciones y aplicaciones, es conveniente introducir en el circuito una pequeña resistencia paralela a la unión. En este caso la resistencia equivalente de la unión es pequeña e independiente del voltaje. Esto produce un valor grande de $\Gamma$, el límite sobreamortiguado del modelo RCSJ es apropiado y la curva IV no muestra histéresis (Fig. 1.5 con $\Gamma=5$ ).

Por último, merece la pena mencionar que en algunos casos, fundamentalmente cuando se trata con uniones pequeñas, para describir el comportamiento del sistema es esencial considerar también la impedancia del circuito externo.

\subsubsection{Uniones Josephson "largas"}

Una unión Josephson larga (long Josephson junction) es una unión (Fig. 1.7) en la cual una dimensión (sea $x$ ) es grande con respecto a la llamada longitud de penetración de Josephson [7]. En este caso la diferencia de fase no puede ser considerada constante en toda la unión y depende también de la coordenada espacial, por lo que se expresa como $\varphi(x, t)$.

La electrodinámica de la unión se describe en este caso por una ecuación nolineal en derivadas parciales que, despreciando efectos disipativos, puede ser escrita como

$$
\varphi_{x x}-\varphi_{t t}=\sin \varphi
$$

Dicha ecuación se corresponde a la llamada ecuación de sine-Gordon, popular por soportar solitones, soluciones coherentes localizada tipo partícula del sistema. La variable $\varphi(x)$ se corresponde tanto con la diferencia de fase como con el flujo de campo magnético normalizado. Entonces, un solitón en la unión corresponde a una solución en la que la fase cambia de cero to $2 \pi$; o el flujo de cero a $\Phi_{0}$; esto es, un cuanto de flujo magnético o fluxón.

Si incluimos disipación y una corriente externa, la dinámica del sistema es descrita por una ecuación de sine-Gordon perturbada

$$
\varphi_{x x}-\varphi_{t t}-\sin \varphi=\alpha \varphi_{t}-\beta \varphi_{x x t}-\gamma
$$




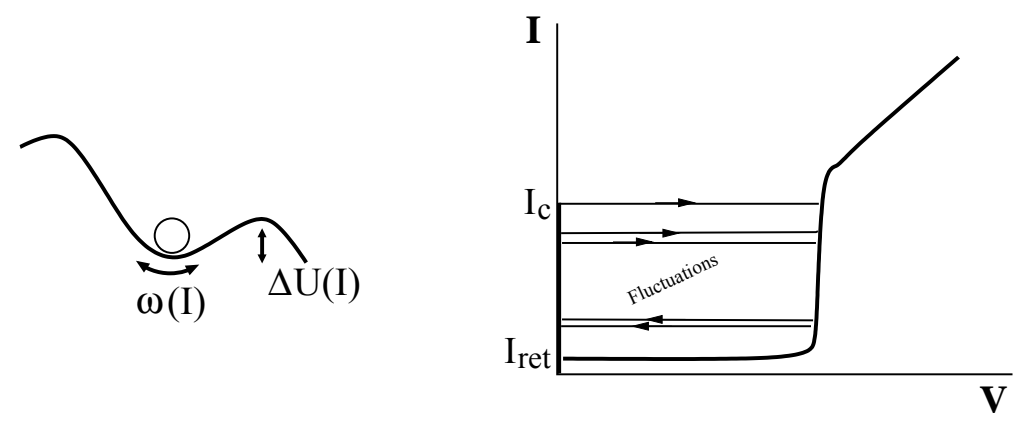

Figura 1.8: El escape de una unión del estado superconductor en la presencia de fluctuaciones térmicas es un problema análogo al escape de una partícula de un pozo en un potencial coseno inclinado.

En esta tesis no vamos a trabajar sobre modelos de uniones Josephson largas. Sin embargo, una red de uniones Josephson en paralelo puede ser considerada como la versión discreta de una unión Josephson larga. Además, entre otras cosas, estas configuraciones son sistema experimentales ideales para estudiar de las propiedades de solitones, por ello han recibido una atención muy importante desde el campo de la física no lineal. Para aprender más sobre las uniones largas puede consultarse el libro de Barone y Paternó [7], el siguiente artículo de revisión de A. Ustinov [22] y las referencias incluidas en él o el trabajo de Wallraff sobre vórtices cuánticos en uniones largas [23].

\subsection{Efecto de la temperatura}

\subsubsection{Adición de ruido térmico}

Las fluctuaciones térmicas pueden ser incluidas en el modelo mediante la adición de una fuente de corriente ruidosa $\tilde{I}(t) \operatorname{con}\langle\tilde{I}(t)\rangle=0$ y $\left\langle\tilde{I}(t) \tilde{I}\left(t^{\prime}\right)\right\rangle=$ $\left(2 k_{B} T / R\right) \delta\left(t-t^{\prime}\right)$. En este caso la corriente total, en unidades normalizadas, está dada por

$$
i=\mathcal{N}(\varphi)=\ddot{\varphi}+\Gamma \dot{\varphi}+\sin \varphi+\tilde{i}
$$

$\operatorname{con}\langle\tilde{i}(\tau)\rangle=0$ y $\left\langle\tilde{i}(\tau) \tilde{i}\left(\tau^{\prime}\right)\right\rangle=\left(2 \Gamma k_{B} T / E_{J}\right) \delta\left(\tau-\tau^{\prime}\right)$.

\subsubsection{Modelo de salto de barrera y efecto de la temperatura}

En presencia de ruido térmico, cerca de $I_{c}$ la unión puede escapar del estado de voltaje nulo gracias a las fluctuaciones térmicas, y cerca de $I_{\text {ret }}$ puede volver al estado de voltaje cero (Fig. 1.8). 
Un experimento típico es la medida de la curva IV de una unión. En este caso lo que se hace es imponer una rampa de cambio de la corriente y medir el voltaje en la unión para distintos valores de la corriente. La dinámica de la unión es tan rápida (dada por $\omega_{p} \simeq$ centenares de GHz) que la electrónica de medida sólo es capaz de dar el valor medio de la señal del voltaje o voltaje dc de la unión. Buscando el análogo con el problema de una partícula en un potencial periódico inclinado se trata de ir inclinando el potencial poco a poco, con una determinada velocidad y buscar el momento en el cual la partícula, por excitación térmica es capaz de superar la barrera de potencial. Si el amortiguamiento del sistema es suficientemente pequeño una vez la partícula supere la barrera habrá adquirido la energía suficiente para deslizarse por el potencial sin ser atrapada por ninguno de los pozos metaestables restantes.

Este proceso de escape térmico depende de tres variables fundamentalmente: el amortiguamiento, la temperatura y la rampa aplicada. Una rampa lenta posibilita que el escape se observe a valores menores de la corriente. Dado que el proceso de escape térmico es estocástico dicho experimento debe repetirse muchas veces y al final somos capaces de calcular el valor medio de la corriente de escape (o valor medio de la corriente de desanclaje, de depinning, o de switching), su desviación cuadrática media y de hecho la distribución de probabilidad completa $P(I)$.

Esta distribución de probabilidad se puede relacionar con la tasa de escape de una partícula de un potencial metaestable $r(I)$ que básicamente mide el tiempo de vida media de una partícula en el pozo metaestable de potencial (su inverso) o el flujo de partículas fuera del pozo de potencial. En nuestro caso ese potencial está dado por $U(I) / E_{J}=-\cos \varphi-\left(I / I_{c}\right) \varphi$.

La distribución de corrientes de escape $P(I)$ está relacionada con la tasa de escape $r(I)$ a través del siguiente argumento [24, 25, 26]. Sea la corriente 0 a tiempo $t=0$ e incrementemos esta con una tasa constante dada por $\dot{I}$. La probabilidad $W(I(t))$ de que el sistema persista en el estado metaestable a tiempo $t$ viene dada por

$$
W(I(t))=\exp \left[-\int_{0}^{t} r\left(I\left(t^{\prime}\right)\right) d t^{\prime}\right]
$$

Realizando un cambio de variable de $t^{\prime}$ a $I\left(t^{\prime}\right)$ tenemos

$$
P(I)=-\frac{d}{d I} W(I)=\frac{r(I)}{\dot{I}} \exp \left[-\int_{0}^{I} \frac{r\left(I^{\prime}\right)}{\dot{I}} d I^{\prime}\right]
$$

A partir de aquí podemos calcular el valor medio de la corriente de escape o su desviación cuadrática media por ejemplo. 
Otra expresión útil e igualmente válida es la siguiente [25]

$$
P(I)=\frac{r(I)}{\dot{I}}\left(1-\int_{0}^{I} P(u) d u\right)
$$

\subsubsection{El "problema de Kramers" y uniones Josephson}

Bajo el sobrenombre de "problema de Kramers" (Kramers problem) se conoce el problema de encontrar la tasa de escape de una partícula fuera de un potencial metaestable. Dicho problema juega un papel fundamental en muchas áreas de la física incluyendo la física de bajas temperaturas, la física nuclear y la físico-química. [27, 28, 29]

Como acabamos de ver, existe una relación directa entre la medida de la función de distribución de probabilidad $P(I)$, probabilidad de que una unión salte al estado óhmico a un cierto valor de la corriente cuando se somete a una rampa uniforme de corriente externa, y el valor de la tasa de escape $r(I)$, inverso del tiempo medio de persistencia de la unión en el estado superconductor. Esta relación nos indica por lo tanto que las uniones Josephson son un sistema ideal donde medir la tasa de escape de un sistema y confrontar los resultados con los resultados teóricos existentes. La comprensión de dicho fenómeno, tanto en el caso clásico como en el cuántico del mismo, para el caso de uniones pequeñas donde el mecanismo fundamental de escape es por efecto túnel ha sido objeto del trabajo de muchos grupos de investigación [24, 25, 30, 31, 32, 33, 34, 35, 10].

Desde los trabajos de Martinis, Devoret y Clarke fundamentalmente [34] se asume que la tasa de escape $r_{\text {esc }}$ en el régimen clásico puede ser aproximada por

$$
r_{\mathrm{esc}}=a_{t} \frac{\omega}{2 \pi} \exp \left(-\frac{\Delta U}{k_{B} T}\right),
$$

donde $a_{t}$ es un prefactor que depende del valor del amortiguamiento [28, 29] y que en el régimen de amortiguamiento moderado-bajo puede ser aproximado por el resultado de Büttiker, Harris y Landauer [36]

$$
a_{t}=4 \alpha /\left[\left(1+\alpha k_{B} T / 1.8 \Gamma \Delta U\right)^{1 / 2}+1\right]^{2}
$$

aquí $\alpha$ es un coeficiente en torno a la unidad.

Dicho resultado es correcto para un cierto rango de valores del amortiguamiento. El último capítulo de este trabajo de tesis doctoral está dedicado a un estudio numérico detallado del valor de la tasa de escape de un sistema en un amplio rango de valores de amortiguamiento. Dichos resultados numéricos serán confrontados frente a numerosos resultados teóricos y se estudiará el rango de validez de los mismos. 


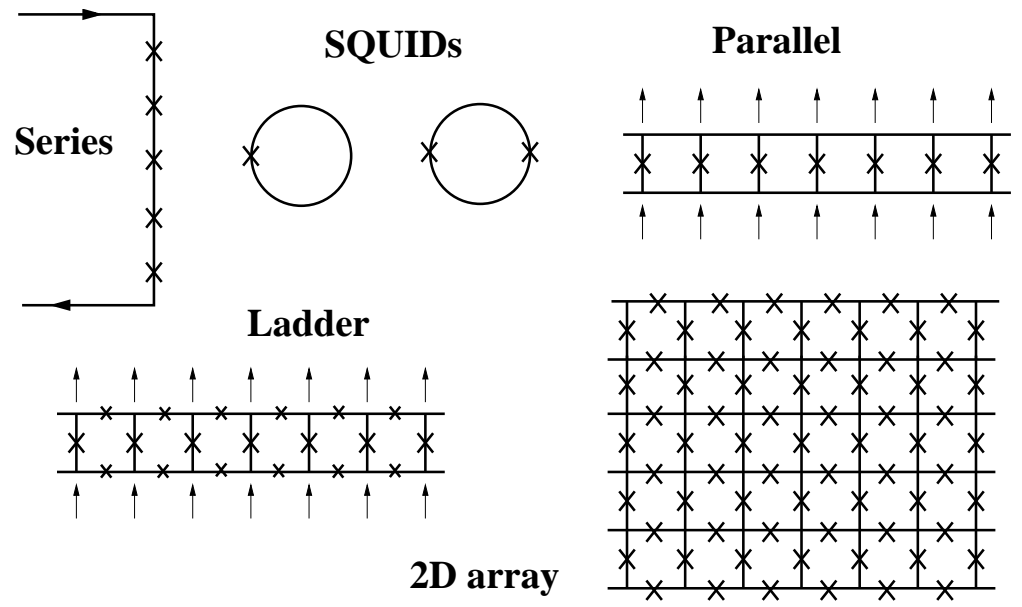

Figura 1.9: Esquemas de diferentes tipos de redes de uniones Josephson. Cada cruz representa una unión Josephson.

\subsection{Redes de uniones Josephson}

Los sistemas hechos con superconductores interrumpidos por uniones Josephson son conocidos habitualmente como redes de uniones Josephson. La figura 1.9 muestra algunos ejemplos de tales arreglos. Todos ellos son fáciles de fabricar y han sido estudiados ampliamente. El primer dispositivo consiste en una red de uniones Josephson en serie. Este tipo de redes han sido empleadas para estudiar fenómenos de sincronización de fase y diseñar el estándar de voltaje [37]. Los anillos superconductores interrumpidos por una o dos uniones se conocen como SQUIDs (de superconducting quantum interference device). Los SQUIDs proporcionan una medida muy sensible de flujo magnético y hoy son utilizados en muchos laboratorios como aparatos estándar de medida de campo magnéticos $[7,8,38,39,40]$. Las redes con uniones acopladas en paralelo se han diseñado para estudiar propiedades de transporte de fluxones pero desde un punto de vista más fundamental son interesantes por constituir una realización experimental del modelo Frenkel-Kontorova (también conocido como modelo sine-Gordon discreto ${ }^{2}$ ) [41, 42, 43, 44, 45]. Las redes en escalera ("ladder") se han diseñado para estudiar la transición de modelos en una dimensión a modelos bidimensionales y han permitido llevar a cabo la observación experimental de los modos intrínsecos localizados o "breathers" discretos [46, 47, 48, 49, 50]. Los arreglos bidimensionales de uniones son sistemas modelo ideales para estudiar transiciones de fase en dimensión dos y los efectos de frustración y

\footnotetext{
${ }^{2}$ Según esta perspectiva, las redes de uniones Josephson en paralelo, que son los dispositivos objeto de esta tesis doctoral, pueden ser considerados como versiones discretizadas de una unión Josephson larga.
} 
desorden, la dinámica de vórtices, sincronización de fase y otros resultados de dinámica no lineal [51].

Dado un arreglo de uniones Josephson, para deducir las ecuaciones de la dinámica del sistema tenemos que aplicar las leyes de Kirchoff para corriente y voltaje y la cuantización del fluxoide. La cuantización del fluxoide establece que para cualquier camino cerrado $l$ en la red (con al menos una unión) la suma de la diferencias de fase a lo largo del camino está dada por

$$
\sum_{j \in l} \varphi_{j}=2 \pi\left(n_{l}-f_{l}\right)
$$

El entero $n_{l}$ es la vorticidad del camino y su origen es que la fase $\theta$ de la función de ondas en cada isla superconductora está multivaluada. $f_{l}$ describe el flujo a través del camino debido al campo magnético total (externo más inducido) y medido en unidades de $\Phi_{0}\left(f_{l}=\Phi_{l} / \Phi_{0}\right)$. En general, para calcular el flujo total inducido en una celda debemos tener en cuenta la matriz total de inducciones del circuito. Sin embargo, en muchos casos podemos estudiar el sistema en una aproximación más sencilla y considerar sólo la autoinducción $L$ de cada celda. El parámetro $\lambda=\Phi_{0} / 2 \pi I_{c} L$ mide la importancia de los campos inducidos.

Podemos prescindir de los términos $n_{l}^{3}$ y escribir

$$
\sum_{j \epsilon l} \varphi_{j}=-2 \pi\left(f_{l}^{\text {ext }}+f_{l}^{\text {ind }}\right)=-2 \pi f_{l}^{\text {ext }}-\frac{1}{\lambda} \frac{I^{\text {loop }}}{I_{c}} .
$$

Dependiendo de la importancia de los campos inducidos, los circuitos Josephson pueden ser divididos en dos tipos generales. Los circuitos de un primer tipo tienen $\lambda \gg 1$ de modo que los campos inducidos no son importantes (estos circuitos normalmente son hechos con aluminio). Por otro lado, en los circuitos que pertenecen al segundo tipo los campos inducidos por las corrientes que circulan son importantes (estos circuitos son normalmente hechos de niobio).

Si los efectos inductivos pueden despreciarse, la cuantización del fluxoide (Eq. 1.22) impone una serie de ligaduras a las ecuaciones y reduce el número de variables independientes del sistema.

\subsubsection{Redes en serie}

La figura 1.10 muestra una red de uniones Josephson acopladas en serie, alimentadas por una corriente alterna y un circuito externo de carga. Las ecua-

\footnotetext{
${ }^{3}$ En el modelo RCSJ las ecuaciones dinámicas dependen sólo de $\ddot{\varphi}, \dot{\varphi}$ y $\sin \varphi$. Por lo tanto las ecuaciones son independientes de los $n_{l}$ y podemos eliminarlos de las mismas
} 


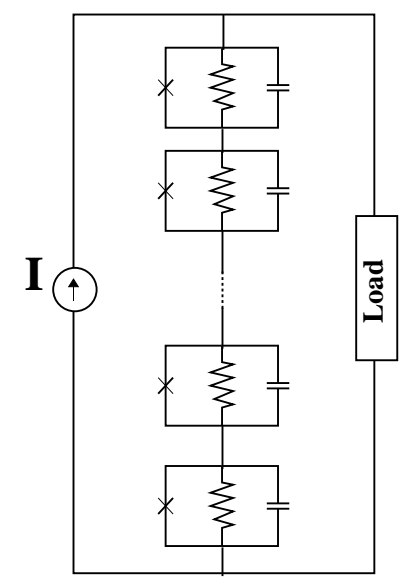

Figura 1.10: Red de uniones Josephson en serie, con corriente externa y circuito externo de carga.

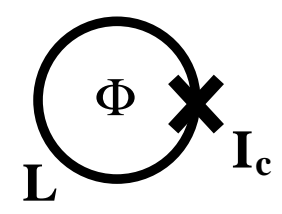

Figura 1.11: Dispositivo rf-squid

ciones de la red pueden escribirse como [52]

$$
\begin{array}{r}
\ddot{\varphi}_{k}+\Gamma \dot{\varphi}_{k}+\sin \varphi_{k}+I_{L}(t)=I \\
V(t)=\sum_{k=1}^{N} \dot{\varphi}_{k}=F\left(I_{L}(t)\right) .
\end{array}
$$

Las uniones en serie se comportan como elementos independientes alimentados por una misma corriente y acoplados a través del circuito externo.

\subsection{2. $\quad$ rf-SQUID}

Este dispositivo está formado por un anillo superconductor interrumpido por una unión Josephson (Fig. 1.11). Su comportamiento está gobernado por el valor del flujo total a través del SQUID, $\Phi$. A partir de la condición de cuantización del fluxoide $\varphi=-2 \pi \frac{\Phi}{\Phi_{0}}$ se tiene,

$$
\Phi=\Phi_{\text {ext }}-L I_{c} \sin 2 \pi \frac{\Phi}{\Phi_{0}}
$$




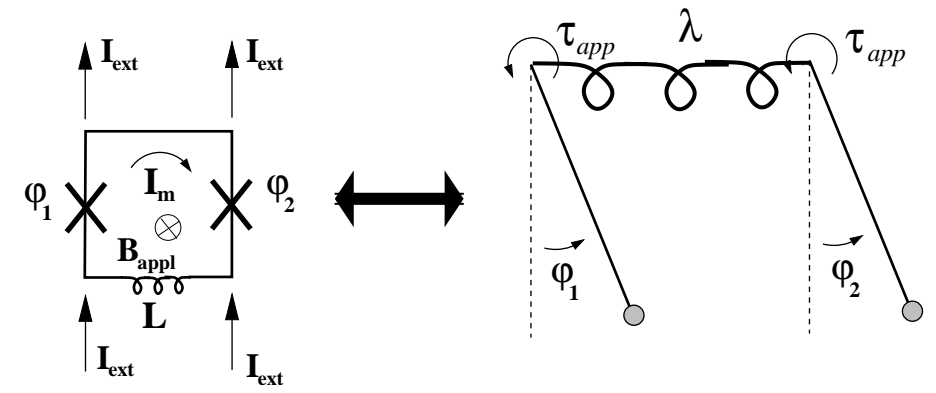

Figura 1.12: Dispositivo dc-squid y equivalencia con un sistema formado por dos péndulos acoplados.

y la energía potencial del sistema está dada por

$$
U(\Phi)=-E_{J} \cos 2 \pi \frac{\Phi}{\Phi_{0}}+\frac{\left(\Phi-\Phi_{\text {ext }}\right)^{2}}{2 L} .
$$

Ya que en este circuito no hay corriente externa, el SQUID es operado acoplado a un circuito de radio-frecuencias (resonador).

Además de como detector de campos magnético los rf-SQUIDs son importantes para el estudio de problemas fundamentales de mecánica cuántica (ver Ref. [53] y las referencias contenidas allí).

\subsection{3. dc-SQUID}

Este dispositivo consiste en un anillo superconductor interrumpido por dos uniones Josephson (Fig. 1.12). Para la corriente circulando por cada unión tenemos:

$$
\begin{aligned}
& i_{1}=\ddot{\varphi}_{1}+\Gamma \dot{\varphi}_{1}+\sin \varphi_{1}=i^{\text {mesh }}+i_{\text {ext }}, \\
& i_{2}=\ddot{\varphi}_{2}+\Gamma \dot{\varphi}_{2}+\sin \varphi_{2}=-i^{\text {mesh }}+i_{\text {ext }} ;
\end{aligned}
$$

y la cuantización del fluxoide:

$$
\left(\varphi_{1}-\varphi_{2}\right)=-\frac{2 \pi}{\Phi_{0}}\left(B_{\text {appl }} S+L I^{\text {mesh }}\right) .
$$

Normalizando, tenemos

$$
i^{\text {mesh }}=-\lambda\left(\varphi_{1}-\varphi_{2}+2 \pi f_{0}\right)
$$

$\left(f_{0}=B_{\mathrm{appl}} S / \Phi_{0}\right)$.

Entonces las ecuaciones para la dinámica del arreglo son

$$
\begin{aligned}
& \ddot{\varphi}_{1}+\Gamma \dot{\varphi}_{1}+\sin \varphi_{1}=-\lambda\left(\varphi_{1}-\varphi_{2}+2 \pi f_{0}\right)+i_{\mathrm{ext}} \\
& \ddot{\varphi}_{2}+\Gamma \dot{\varphi}_{2}+\sin \varphi_{2}=\lambda\left(\varphi_{1}-\varphi_{2}+2 \pi f_{0}\right)+i_{\mathrm{ext}},
\end{aligned}
$$



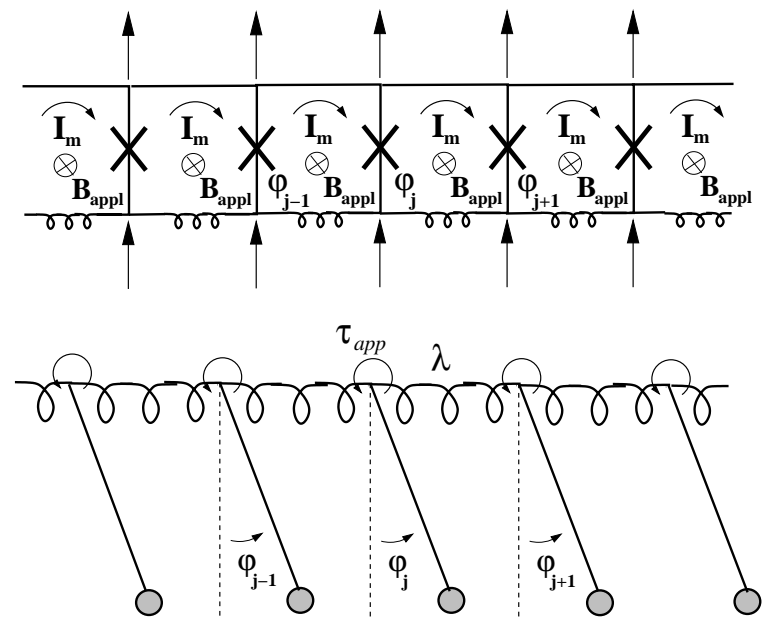

Figura 1.13: Red de uniones Josephson en paralelo

donde $I_{\text {ext }}=I_{\text {total }} / 2$. Estas ecuaciones muestran que el problema de dos uniones conectadas en paralelo por un elemento autoinductivo es equivalente al problema de dos péndulos acoplados por un muelle de torsión (ver Fig. 1.12).

Si los efectos inductivos pueden ser despreciados, la cuantización del fluxoide impone una ligadura sobre las fases y tenemos

$$
\varphi_{1}-\varphi_{2}=-2 \pi f_{0}
$$

En este caso,

$$
i_{\mathrm{ext}}=\frac{i_{1}+i_{2}}{2}=\ddot{\varphi}_{1}+\Gamma \dot{\varphi}_{1}+\cos \left(\pi f_{0}\right) \sin \left(\varphi_{1}+\pi f_{0}\right) .
$$

Dicho sistema se comporta como una única unión cuya corriente crítica, $2 I_{c} \cos \left(\pi f_{0}\right)$, está controlada por el campo magnético externo.

\subsubsection{Red de uniones Josephson en paralelo}

Una red de uniones Josephson en paralelo está formada por un conjunto de uniones conectadas en paralelo mediante "cables superconductores". La analogía mecánica de este sistema es un conjunto de péndulos conectados por muelles de torsión (ver Fig 1.13). Una consecuencia importante de esta interacción armónica entre uniones es que todas tienen el mismo voltaje dc.

Las ecuaciones de la dinámica de la red pueden ser generalizadas fácilmente a partir de las ecuaciones del SQUID dc. Para la red paralela de $N$ uniones

$$
\ddot{\varphi}_{j}+\Gamma \dot{\varphi}_{j}+\sin \varphi_{j}=\lambda\left(\varphi_{j+1}-2 \varphi_{j}+\varphi_{j-1}\right)+i_{\text {ext }}
$$




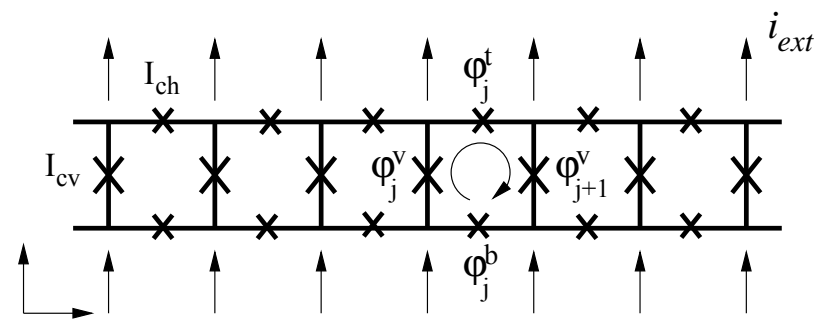

Figura 1.14: Escalera de uniones Josephson

con $j=1, \ldots N$. Las condiciones de frontera están dadas por $\varphi_{0}=\varphi_{1}-2 \pi f_{0}$ y $\varphi_{N+1}=\varphi_{N}+2 \pi f_{0}$ para el caso de redes con fronteras abiertas $\varphi_{N+1}=$ $\varphi_{1}+2 \pi n_{k}$ y $\varphi_{0}=\varphi_{N}-2 \pi n_{k}$ para redes en anillo. En este caso el entero $n_{k}$ cuenta el número de kinks o fluxones atrapados en la red.

Las ecuaciones (1.34) son también las ecuaciones de la dinámica del modelo Frenkel-Kontorova forzado y amortiguado o de la ecuación de sine-Gordon discreta. El trabajo realizado en esta tesis doctoral considera una red de uniones en paralelo con geometría de anillo lo que posibilita que los fluxones queden atrapados en su interior.

\subsubsection{La escalera de uniones Josephson}

Una escalera de uniones Josephson (Fig. 1.14) es una red cuasi-unidimensional que se consigue cuando los cables horizontales de la red en paralelo son sustituidos por uniones Josephson. Podemos pensar en este sistema en términos de un conjunto de péndulos (las uniones verticales) conectadas en paralelo por muelles no convexos (las uniones horizontales). Como consecuencia de las interacciones no convexas, una de las diferencias más importantes con respecto a la red paralela es que ahora las uniones verticales no están constreñidas a tener un mismo voltaje dc. Además los cuantos de fluxoide ahora pueden entrar en la red o escapar de la red a través de las uniones horizontales.

Consideremos el caso de una escalera anisótropa. Entonces las corriente críticas de las uniones en la dirección vertical son diferentes de las corrientes críticas de las uniones en la dirección horizontal. Esto puede hacerse fácilmente cambiando el área de las uniones. La corriente crítica, capacidad y conductancia (inversa de la resistencia) de la unión son directamente proporcionales al área de la misma.

Escribiendo las ecuaciones para las corrientes y aplicando la cuantización 


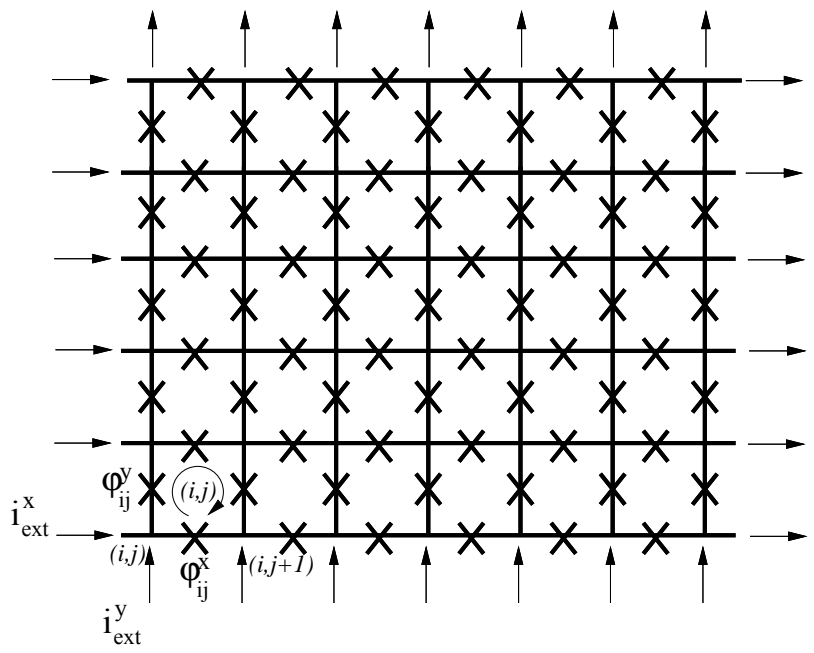

Figura 1.15: Red bidimensional de uniones Josephson

del fluxoide, las ecuaciones de la escalera son

$$
\begin{aligned}
\mathcal{N}\left(\varphi_{j}^{t}\right) & =-\frac{\lambda}{h} \xi_{j}, \\
\mathcal{N}\left(\varphi_{j}^{v}\right) & =\lambda\left(\xi_{j-1}-\xi_{j}\right)+i_{\mathrm{ext}}, \\
\mathcal{N}\left(\varphi_{j}^{b}\right) & =\frac{\lambda}{h} \xi_{j} .
\end{aligned}
$$

Aquí hemos definido

$$
\xi_{j}=-2 \pi f_{j}^{\text {ind }}=\varphi_{j}^{v}+\varphi_{j}^{t}-\varphi_{j+1}^{v}-\varphi_{j}^{b}+2 \pi f_{0},
$$

donde $\xi_{0}=\xi_{N}=0$. Para una escalera con $N$ uniones verticales, $j$ va de 1 to $N$ para las uniones verticales y de 1 a $N-1$ para las horizontales. Hemos normalizado las ecuaciones respecto a los parámetros de las uniones verticales. Así, $h=I_{c h} / I_{c v}=C_{h} / C_{v}=R_{v} / R_{h}$ and $\lambda=\lambda_{v}=\Phi_{0} / 2 \pi I_{c v} L\left(\lambda / h=\lambda_{h}=\right.$ $\left.\Phi_{0} / 2 \pi I_{c h} L\right)$.

\subsubsection{Redes bidimensionales}

La figura 1.15 muestra un diagrama de un red bidimensional cuadrada de uniones Josephson. Siguiendo nuestra aproximación al modelado de las uniones, las uniones están acopladas mediante la condición de la cuantización del fluxoide con la inclusión de tan sólo campos magnéticos autoinducidos en cada celda. En esta aproximación las ecuaciones de la dinámica de la red están 
dadas por:

$$
\begin{aligned}
\mathcal{N}\left(\varphi_{i j}^{x}\right) & =\frac{\lambda}{h}\left(\xi_{i j}-\xi_{i j-1}\right)+\frac{i_{x}^{\mathrm{ext}}}{h} \\
\mathcal{N}\left(\varphi_{i j}^{y}\right) & =\lambda\left(\xi_{i-1 j}-\xi_{i j}\right)+i_{y}^{\text {ext }}
\end{aligned}
$$

$\xi_{i j}$ mide la intensidad de los campos inducidos

$$
\xi_{i j}=-2 \pi f_{i j}^{\mathrm{ind}}=\varphi_{i j}^{y}+\varphi_{i j+1}^{x}-\varphi_{i+1 j}^{y}-\varphi_{i j}^{x}+2 \pi f_{0}
$$

y hemos normalizado con respecto a los parámetros de las uniones verticales. Así $h=I_{c x} / I_{c y}=C_{x} / C_{y}=R_{y} / R_{x}$ y $\lambda=\lambda_{y}=\Phi_{0} / 2 \pi I_{c y} L\left(\lambda / h=\lambda_{x}=\right.$ $\left.\Phi_{0} / 2 \pi I_{c x} L\right)$.

Este es el modelo de una red bidimensional cuando sólo las auto-inducciones se tienen en cuenta. En función del problema que se quiera estudiar en algunas ocasiones es necesario incluir la matriz total de inducciones, en otros casos las autoinducciones pueden despreciarse.

En general, en una red cuadrada bidimensional $(\mathrm{N} \times \mathrm{N})$ tenemos que resolver las ecuaciones para la dinámica de $2 N^{2}-2 N$ (para condiciones de contorno libres) diferencias de fase invariante gauge, $\varphi_{i j}$. Sin embargo, cuando los campos inducidos pueden despreciarse (límite $\lambda \gg 1$ ), la condición de cuantización del fluxoide impone $(N-1)^{2}$ ligaduras sobre esas variables. Entonces, es más conveniente expresar las ecuaciones del sistema en función de las fases en cada isla $\theta_{i}$, que son $N^{2}-1$ variables independientes. Esto se consigue escribiendo $\varphi_{i j}=\theta_{i}-\theta_{j}-A_{i j}$ [donde (para un gauge dado) $\operatorname{los} A_{i j}=\frac{2 \pi}{\Phi_{0}} \int_{i}^{j} \vec{A}(\vec{r}, t) \overrightarrow{d l}$, dependen sólo del campo magnético externo] y las ecuaciones dinámicas resultan de aplicar la conservación de la corriente.

En el límite $E_{J} \gg E_{C}$ del sistema la energía Josephson total es la contribución energética relevante del sistema:

$$
H_{J}=-\sum_{<i j>} E_{J} \cos \left(\theta_{i}-\theta_{j}-A_{i j}\right)
$$

En este caso una red bidimensional de uniones Josephson constituye una realización experimental del modelo XY $\left(A_{i j}=0\right)$ o del modelo XY frustrado $\left(A_{i j} \neq 0\right)$, por lo que constituye un sistema experimental excepcional para el estudio de este modelo fundamental de la física estadística [51]. 


\section{Capítulo 2}

\section{El anillo de uniones Josephson}

El objetivo fundamental de este trabajo de tesis doctoral es el estudio de la dinámica de fluxones en anillos de uniones Josephson en la presencia de ruido térmico, y en particular de las propiedades de desanclaje (depinning) y salto a la rama óhmica (switching) de los mismos. Nuestro sistema es un anillo de uniones Josephson alimentado por una corriente externa. Un anillo de uniones Josephson es una red formada por una serie de uniones acopladas en paralelo y cerrada formando un anillo (FIg, 2.1). Debido a la cuantización del flujo magnético, en el interior del anillo el flujo total es igual a un número entero de cuantos de flujo magnético. Un experimento típico consiste en enfriar en presencia de un campo magnético el anillo superconductor por debajo de la temperatura crítica del mismo. El campo aplicado crea un determinado flujo en el interior del anillo. A continuación se procede a eliminar el campo externo. Entonces, el superconductor responde induciendo corrientes superconductoras en el sistema que compensan el campo retirado. De este modo que el flujo magnético en el anillo queda atrapado a la par que cuantizado.

En este capítulo presentaremos las ecuaciones que describen la dinámica de un anillo de uniones Josephson. Veremos la conexión del sistema con algunos sistemas-modelo de interés para la física no-lineal y la física estadística. Así mismo realizaremos una breve presentación de los estudios de movimiento de partículas en potenciales asimétricos y veremos como es posible diseñar anillos tales que el potencial efectivo que ve el fluxón es un potencial asimétrico.

\subsection{Ecuaciones de la dinámica del sistema}

Consideremos una red de $N$ uniones Josephson acopladas en paralelo, alimentado por una corriente externa total $I_{\text {total }}^{\text {ext }}$ y cerrada en forma de anillo 

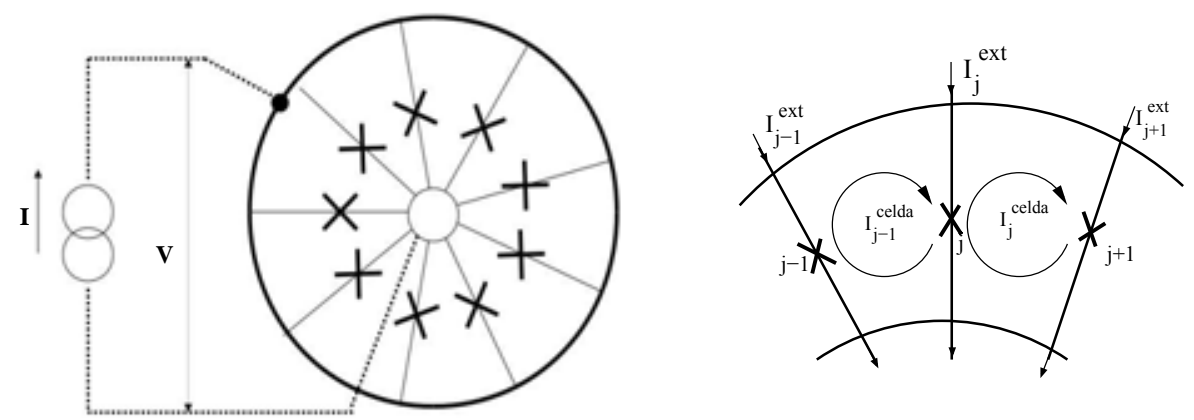

Figura 2.1: Arreglo superconductor con 9 uniones Josephson en configuración de anillo. Las corrientes superconductoras circulantes por el superconductor exterior son las responsables del atrapamiento de flujo magnético en el sistema.

(ver figura 2.1). Todas las uniones Josephson son del mismo tipo; esto es, están fabricadas en un mismo proceso, con los mismos materiales y según una misma tecnología, pero la flexibilidad del diseño permite que el área varíe de una unión a otra y que las celdas formadas entre uniones vecinas sean diferentes. El proceso de fabricación fija la densidad de corriente crítica, la capacidad específica y la resistividad de la unión siendo $I_{c}=A J_{c}, C=A \sigma$ y $R=\rho / A$. Por lo tanto, las uniones de distinta áreas tienen distinta corriente crítica, capacidad y resistencia normal pero un mismo valor de la frecuencia de plasma $\omega_{p}=\sqrt{\left(2 \pi I_{c} / \Phi_{0} C\right)}$ y el parámetro de Stewart-McCumber o amortiguamiento efectivo $\Gamma=1 / \beta_{c}^{1 / 2}=\sqrt{\left(\Phi_{0} / 2 \pi C R^{2} I_{c}\right)}$. Con respecto a la disparidad de tamaños de celda su consecuencia principal es una diferencia en los valores de la autoinducción de cada celda $L$ y en el flujo debido al campo magnético externo aplicado $\Phi^{\text {ext }}=B^{\text {ext }} S_{\text {celda }}$.

Tal y como hemos expuesto en el capítulo anterior las propiedades físicas más sobresalientes del sistema pueden ser calculadas a partir del valor de las diferencia de fase invariante gauge $\varphi_{j}, j=1, \ldots N$, de las uniones. Así por ejemplo el voltaje entre el lado externo e interno del anillo es $V=V_{j}$ con $V_{j}$ la caída de voltaje en cada unión, y la corriente total aplicada al sistema $I=\sum_{j} I_{j}$ con $I_{j}$ la corriente que circula por cada unión. Para escribir las ecuaciones de la dinámica de las fases utilizaremos el modelo RCSJ de la unión, con la incorporación del efecto de la temperatura introduciendo una fuente de corriente ruidosa $\tilde{I}(t)$. Con respecto al anillo, podemos utilizar un modelo de corriente de rama o de corrientes de malla y aplicar las ecuaciones de Kirchoff y la cuantización del fluxoide. En el marco de corrientes de malla podemos escribir:

$$
I_{j}=\frac{\hbar}{2 e} C_{j} \frac{d^{2} \varphi_{j}}{d t^{2}}+\frac{\hbar}{2 e R_{j}} \frac{d \varphi_{j}}{d t}+I_{c j} \sin \varphi_{j}+\tilde{I}_{j}(t)=I_{j}^{\text {ext }}+I_{j-1}^{\text {celda }}-I_{j}^{\text {celda }} .
$$


La corriente total que circula a través de la unión $j, I_{j}$, puede describirse en término de las corrientes de celda definidas en las celdas $j-1$ y $j$ y de la corriente externa aplicada a la unión $j, I_{j}^{\text {ext }}$, con $\sum_{j} I_{j}^{\text {ext }}=I_{\text {total }}^{\text {ext }}($ ver figura 2.1).

Las fluctuaciones térmicas están representadas en la ecuación anterior por los términos $\tilde{I}_{j}(t)$, cumpliéndose $\left\langle\tilde{I}_{j}(t)\right\rangle=0$ y $\left\langle\tilde{I}_{j}(t) \tilde{I}_{k}\left(t^{\prime}\right)\right\rangle=\left(2 k_{B} T / R_{j}\right) \delta_{j k} \delta(t-$ $\left.t^{\prime}\right)$.

Por otro lado el flujo magnético en cada celda $j$ debido a los campos inducidos por las corrientes que circulan en el sistema está dado por

$$
\Phi_{j}^{\text {ind }}=\sum_{k} M_{j k} I_{k}^{\text {celda }}+\sum_{k} \widehat{M}_{j k} I_{k}^{\text {ext }} \simeq L_{j} I_{j}^{\text {celda }}+\widehat{L}_{j+1} I_{j+1}^{\text {ext }}-\widehat{L}_{j} I_{j}^{\text {ext }}
$$

La matriz $M$ es la matriz de inducciones de las corrientes de celda, la matriz $\widehat{M}$ es la matriz de inducciones de las corrientes externas. En ambos casos nos quedaremos en la aproximación de menor orden, representada por los términos $L$ y $\widehat{L}$.

La condición de la cuantización del fluxoide aplicada a cada celda establece

$$
\begin{aligned}
\varphi_{j+1}-\varphi_{j} & =-2 \pi\left(f_{j}^{\text {ext }}+f_{j}^{\text {ind }}\right)=-2 \pi\left(\frac{\Phi_{j}^{\text {ext }}}{\Phi_{0}}+\frac{\Phi_{j}^{\text {ind }}}{\Phi_{0}}\right)= \\
& =-\frac{2 \pi}{\Phi_{0}}\left(\Phi_{j}^{\text {ext }}+L_{j} I_{j}^{\text {celda }}+\widehat{L}_{j+1} I_{j+1}^{\text {ext }}-\widehat{L}_{j} I_{j}^{\text {ext }}\right)
\end{aligned}
$$

con lo que

$$
\begin{aligned}
I_{j}^{\text {celda }} & =-\frac{\Phi_{0}}{2 \pi L_{j}}\left(\varphi_{j+1}-\varphi_{j}\right)-\frac{1}{L_{j}}\left(\Phi_{j}^{\text {ext }}+\widehat{L}_{j+1} I_{j+1}^{\text {ext }}-\widehat{L}_{j} I_{j}^{\text {ext }}\right)= \\
& =-\frac{\Phi_{0}}{2 \pi L_{j}}\left(\varphi_{j+1}-\varphi_{j}\right)-G_{j},
\end{aligned}
$$

donde hemos definido los términos $G_{j}$ :

$$
G_{j}=\frac{1}{L_{j}}\left(\Phi_{j}^{\mathrm{ext}}+\widehat{L}_{j+1} I_{j+1}^{\mathrm{ext}}-\widehat{L}_{j} I_{j}^{\mathrm{ext}}\right)=\frac{1}{L_{j}}\left(\Phi_{j}^{\mathrm{ext}}+\widetilde{\Phi}_{j}^{\mathrm{ext}}\right),
$$

que recogen la contribución de campos externos y campos inducidos por la corriente externa. Por lo tanto

$$
I_{j-1}^{\text {celda }}-I_{j}^{\text {celda }}=\frac{\Phi_{0}}{2 \pi L_{j}}\left(\varphi_{j+1}-\varphi_{j}\right)+G_{j}-\frac{\Phi_{0}}{2 \pi L_{j-1}}\left(\varphi_{j}-\varphi_{j-1}\right)-G_{j-1} \text {. }
$$

Entonces, tras la aproximación hecha en (2.2), la ecuación para la corriente total a través de la unión $j$ puede ser escrita de la siguiente manera:

$$
\begin{aligned}
I_{j}=\frac{\hbar}{2 e} C_{j} & \frac{d^{2} \varphi_{j}}{d t^{2}}+\frac{\hbar}{2 e R_{j}} \frac{d \varphi_{j}}{d t}+I_{c j} \sin \varphi_{j}+\tilde{I}_{j}(t)= \\
& =I_{j}^{\text {ext }}+\frac{\Phi_{0}}{2 \pi L_{j}}\left(\varphi_{j+1}-\varphi_{j}\right)-\frac{\Phi_{0}}{2 \pi L_{j-1}}\left(\varphi_{j}-\varphi_{j-1}\right)+G_{j}-G_{j-1} .
\end{aligned}
$$


En esta ecuación el término $G_{j}-G_{j-1}$ recoge la contribución debida a la presencia de los campos externos y los inducidos por las corrientes aplicadas,

A continuación vamos a normalizar nuestro sistema de ecuaciones. Para ello mediremos las corrientes en unidades de $I_{c *}$ (la corriente crítica de una de las uniones o la corriente crítica media por ejemplo) y el tiempo en unidades de $1 / \omega_{p}$ (recordamos que los parámetros $\omega_{p}=\sqrt{2 \pi I_{c j} / \Phi_{0} C_{j}} \mathrm{y}$ $\Gamma=\sqrt{\Phi_{0} / 2 \pi I_{c j} R_{j}^{2} C_{j}}$ son idénticos para todas la uniones). Además definimos $\lambda_{j}=\Phi_{0} / 2 \pi I_{c *} L_{j}=L_{J *} / L_{j}$ y usaremos la definición del cuanto de flujo magnético $\left.\Phi_{0}=\frac{\hbar}{2 e}\right)$. Con ello tenemos

$$
\begin{aligned}
& h_{j}\left(\ddot{\varphi}_{j}+\Gamma \dot{\varphi}_{j}+\sin \varphi_{j}+\tilde{i}_{j}\right)= \\
& \quad=i_{j}^{\text {ext }}+\lambda_{j}\left(\varphi_{j+1}-\varphi_{j}\right)-\lambda_{j-1}\left(\varphi_{j}-\varphi_{j-1}\right)+g_{j}-g_{j-1}
\end{aligned}
$$

En esta expresión hemos definido los parámetros $h_{j}=I_{c j} / I_{c *}$. Por otro lado $i_{j}^{\text {ext }}=I_{j}^{\text {ext }} / I_{c *}$ y $g_{j}=G_{j} / I_{c *}$. Por último, respecto el término de corriente ruidosa $\left\langle\tilde{i}_{j}(\tau) \tilde{i}_{k}\left(\tau^{\prime}\right)\right\rangle=\left(2 k_{B} T / R_{j} I_{c *}^{2} \omega_{p}\right) \delta_{j k} \delta\left(\tau-\tau^{\prime}\right)=2 h_{j} \Gamma \tilde{T} \delta_{j k} \delta\left(\tau-\tau^{\prime}\right)$ con $\tilde{T}=k_{B} T / E_{J *}$ y $E_{J *}=\hbar I_{c *} / 2 e$. Las derivadas $\dot{\varphi}=d \varphi / d \tau$ con $\tau$ el tiempo adimensional $\tau=\omega_{p}$.

Estas ecuaciones son más sencillas en los siguientes casos:

1. Red formada por celdas idénticas. En este caso todos los términos inductivos, que son términos geométricos, son iguales: $L_{j}=L$ y $\widehat{L}_{j}=\widehat{L}$. Entonces

$$
\begin{aligned}
& h_{j}\left(\ddot{\varphi}_{j}+\Gamma \dot{\varphi}_{j}+\sin \varphi_{j}+\tilde{i}_{j}\right)= \\
& \quad=i_{j}^{\text {ext }}+\lambda\left(\varphi_{j+1}-2 \varphi_{j}+\varphi_{j-1}\right)+g_{j}-g_{j-1}
\end{aligned}
$$

con

$$
g_{j}-g_{j-1}=\frac{1}{I_{c *} L}\left(\Phi_{j}^{\text {ext }}-\Phi_{j-1}^{\text {ext }}\right)+\frac{\widehat{L}}{L}\left(i_{j+1}^{\text {ext }}-2 i_{j}^{\text {ext }}+i_{j-1}^{\text {ext }}\right)
$$

2. Si además, como suele ocurrir con normalidad, la corriente externa se aplica y extrae uniformemente, $I_{j}^{\text {ext }}=I_{\text {total }}^{\text {ext }} / N=I$ tenemos

$$
\begin{aligned}
& \quad h_{j}\left(\ddot{\varphi}_{j}+\Gamma \dot{\varphi}_{j}+\sin \varphi_{j}+\tilde{i}_{j}\right)= \\
& \quad=i+\lambda\left(\varphi_{j+1}-2 \varphi_{j}+\varphi_{j-1}+2 \pi f_{j}^{\text {ext }}-2 \pi f_{j-1}^{\text {ext }}\right) .
\end{aligned}
$$

Aquí hemos introducido la notación habitual, $f_{j}=\Phi_{j} / \Phi_{0}$.

3. Si todos los campos magnéticos externos actúan uniformemente sobre el sistema, tenemos que, por tratarse de celdas de igual tamaño, los términos $f_{j}^{\text {ext }}$ son constantes y entonces podemos escribir

$$
h_{j}\left(\ddot{\varphi}_{j}+\Gamma \dot{\varphi}_{j}+\sin \varphi_{j}+\tilde{i}_{j}\right)=i+\lambda\left(\varphi_{j+1}-2 \varphi_{j}+\varphi_{j-1}\right) \text {. }
$$


Esta ecuación describe la dinámica de una red homogénea de uniones Josephson formada por uniones de distinto tamaño y será nuestro punto de partida para el estudio de redes irregulares, de tipo ratchet, y de redes regulares que haremos a lo largo de esta tesis doctoral.

4. Por último, encontramos las ecuaciones para un anillo regular de uniones Josephson, formado por uniones idénticas $\left(h_{j}=1\right)$, celdas iguales, alimentado de manera uniforme, bajo la acción de campos magnéticos externos uniformes, en el marco del modelo RCSJ de la unión y en la aproximación de primer orden en la matriz de inducciones:

$$
\ddot{\varphi}_{j}+\Gamma \dot{\varphi}_{j}+\sin \varphi_{j}+\tilde{i}_{j}=i+\lambda\left(\varphi_{j+1}-2 \varphi_{j}+\varphi_{j-1}\right) \text {. }
$$

Por tratarse de un anillo, debemos imponer condiciones de contorno cerradas en el sistema, así la unión/celda $j+N$ se corresponde con la unión/celda $j$ del sistema. Por estar multivaluadas hay que tener especial cuidado con las fases ya que $\varphi \equiv \varphi+2 \pi M$ con $M$ un número entero, así las condiciones de contorno sobre las fases para el sistema establecen

$$
\varphi_{0}=\varphi_{N}-2 \pi M \text { y } \varphi_{N+1}=\varphi_{1}+2 \pi M,
$$

donde $M$ tiene el significado físico de número de cuantos de flujo magnéticos o fluxones que han sido atrapados en el sistema al enfriar ya que

$$
\sum_{j=1}^{N}\left(\varphi_{j+1}-\varphi_{j}\right)=-2 \pi \sum_{j=1}^{N} \frac{\Phi_{j}^{\text {total }}}{\Phi_{0}}=2 \pi M
$$

\subsection{Energía del sistema}

En esta sección escribiremos las expresiones para la energía del sistema. El término de energía cinética está asociado a la energía almacenada en los condensadores.

$$
E_{k i n}=\sum_{j} \frac{1}{2} C_{j} V_{j}^{2}=\sum_{j} \frac{1}{2} C_{j}\left[\frac{\Phi_{0}}{2 \pi}\left(\frac{d \varphi_{j}}{d t}\right)\right]^{2}
$$

El término de energía potencial viene dado por la energía Josephson de las uniones y los términos de energía magnética, asociados fundamentalmente a los campos inducidos. En la aproximación habitual de autoinducciones, e ignorando términos constantes, podemos escribir

$$
E_{\text {pot }}=\sum_{j} E_{J j}\left(1-\cos \varphi_{j}\right)+\sum_{j} \frac{1}{2} L_{j}\left(I_{j}^{\mathrm{celda}}\right)^{2}-\sum_{j} I_{j}^{\operatorname{ext}}\left(\frac{\Phi_{0} \varphi_{j}}{2 \pi}\right)
$$


El primer término describe la energía Josephson del circuito, el segundo de las inducciones de malla y el tercero la energía de las fuentes de corriente. Respecto al segundo de ellos

$$
\begin{gathered}
\sum_{j} \frac{1}{2} L_{j}\left(I_{j}^{\text {celda }}\right)^{2}= \\
=\sum_{j} \frac{1}{2 L_{j}}\left(\frac{\Phi_{0}}{2 \pi}\right)^{2}\left[\varphi_{j+1}-\varphi_{j}+2 \pi f_{j}^{\text {ext }}+\frac{2 \pi}{\Phi_{0}}\left(\widehat{L}_{j+1} I_{j+1}^{\text {ext }}-\widehat{L}_{j} I_{j}^{\text {ext }}\right)\right]^{2}= \\
=\sum_{j} \frac{1}{2 L_{j}}\left(\frac{\Phi_{0}}{2 \pi}\right)^{2}\left[\varphi_{j+1}-\varphi_{j}+2 \pi\left(f_{j}^{\text {ext }}+\tilde{f}_{j}^{\text {ext }}\right)\right]^{2},
\end{gathered}
$$

donde hemos definido el término $\tilde{f}_{j}^{\text {ext }}$, que recoge la contribución al flujo de los campos inducidos por la corrientes externas.

Si medimos la energía en unidades de $E_{J *}$ tenemos

$$
\begin{aligned}
& \frac{E_{k i n}}{E_{J *}}=\sum_{j} \frac{h_{j}}{2 \omega_{p}^{2}}\left(\frac{d \varphi_{j}}{d t}\right)^{2}=\sum_{j} \frac{h_{j}}{2}\left(\frac{d \varphi_{j}}{d \tau}\right)^{2} \\
& \frac{E_{\text {pot }}}{E_{J *}}=\sum_{j} h_{j}\left(1-\cos \varphi_{j}\right)-\sum_{j} i_{j}^{\text {ext }} \varphi_{j}+ \\
& +\sum_{j} \frac{\lambda_{j}}{2}\left[\varphi_{j+1}-\varphi_{j}+2 \pi\left(f_{j}^{\text {ext }}+\tilde{f}_{j}^{\text {ext }}\right)\right]^{2}
\end{aligned}
$$

Si aplicamos las ecuaciones de Hamilton a este sistema recuperamos las ecuaciones dinámicas del mismo (2.8) para el caso de amortiguamiento y temperaturas nulos.

Para las redes que denominamos homogéneas (celdas idénticas y corrientes y campos magnéticos uniformes) e irregulares (uniones distintas) tenemos:

$$
\frac{E_{p o t}}{E_{J *}}=\sum_{j}\left[h_{j}\left(1-\cos \varphi_{j}\right)+\frac{\lambda}{2}\left(\varphi_{j+1}-\varphi_{j}+2 \pi f^{\mathrm{ext}}\right)^{2}-i \varphi_{j}\right] .
$$

Si además las redes son regulares (uniones idénticas), podemos escribir:

$$
\frac{E_{p o t}}{E_{J *}}=\sum_{j}\left[\left(1-\cos \varphi_{j}\right)+\frac{\lambda}{2}\left(\varphi_{j+1}-\varphi_{j}+2 \pi f^{\mathrm{ext}}\right)^{2}-i \varphi_{j}\right]
$$



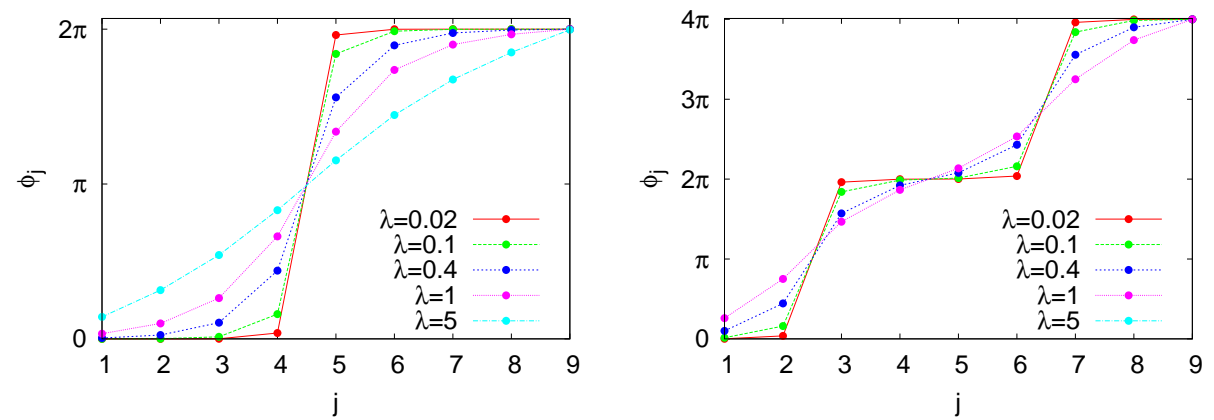

Figura 2.2: Configuración de mínima energía de una configuraqción con 1 fluxón (izquierda) y con 2 fluxones (derecha) para diversos valores del acoplamiento $\lambda$

\subsubsection{Configuraciones de mínima energía}

Las configuraciones de mínima energía del sistema corresponden a soluciones $\left(\delta E_{p o t} / \delta \varphi_{j}\right)=0$ lo cual para una red regular y homogénea implica

$$
\sin \varphi_{j}=\lambda\left(\varphi_{j+1}-2 \varphi_{j}+\varphi_{j-1}\right)-i=0
$$

Dichas ecuaciones deben ser completadas por las adecuadas condiciones de contorno $\varphi_{j+N}=\varphi_{j}+2 \pi M$, lo que se corresponde con una densidad de $M / N$ fluxones o cuantos de flujo magnético en el anillo.

En el caso más sencillo $M=0$, la configuración de mínima energía se corresponde con la configuración uniforme $\varphi_{j}=\varphi^{*}=\sin ^{-1} i$. Dicho estado de equilibrio existe siempre que $|i| \leq 1$.

El estado $M=1$ corresponde a la situación en la que un fluxón o un cuanto de flujo magnético ha quedado atrapado en el interior del anillo superconductor. Entonces las fases deben acumular un giro de $2 \pi$ a lo largo del anillo $\left(\varphi_{j+N}=\right.$ $\left.\varphi_{j}+2 \pi\right)$. La figura 2.2 muestra una configuración de mínima energía para el caso de un fluxón en una red de 9 uniones a $i=0$. Se presenta el resultado para cinco valores distintos de $\lambda$. En el límite $\lambda \rightarrow 0$ la energía Josephson domina y la configuración tiende a un escalón. En el límite $\lambda \rightarrow \infty$ la energía magnética domina y la configuración tiende a un cambio uniforme de las fases $\varphi_{j+1}-\varphi_{j}=2 \pi / N$. En el capítulo 3 investigaremos las propiedades principales de configuraciones de un fluxón.

Valores mayores de $M$ se corresponden con tener varios fluxones en la red. Los fluxones interaccionan repulsivamente entre sí, así que tienden a repartirse uniformemente en el anillo. En anillos suficientemente grandes existen muchas posiciones de equilibrio metaestable para el sistema. 


\subsection{Anillo de uniones Josephson y física no lineal}

\subsubsection{Modelo Frenkel-Kontorova (FK)}

El modelo Frenkel-Kontorova es uno de los modelos paradigmáticos de la física de sistemas discretos no lineales. Introducido primero por Prandtl [54] en 1928 y Dehlinger [55] en 1929, fue estudiado independientemente años después por Frenkel y Kontorova [56] en el contexto del estudio de las dislocaciones en sólidos. Desde entonces ha sido empleado para el estudio de numerosos sistemas físicos y sigue suscitando interés en la actualidad. Braun y Kivshar publicaron en 2004 un exhaustivo libro titulado The Frenkel-Kontorova model. Concepts. methods and aplications [45] que supone una excelente introducción al mismo.

El modelo en su versión estándar describe una cadena de partículas con interacción armónica a primeros vecinos y en la presencia de potencial externo periódico sinusoidal.

$$
\begin{aligned}
& H=T+V\left(x_{j}\right)+W\left(x_{j+1}-x_{j}\right)= \\
& =\sum_{j} \frac{m}{2}\left(\frac{d x_{j}}{d t}\right)^{2}+\sum_{j} \frac{V_{0}}{2}\left[1-\cos \left(\frac{2 \pi x_{j}}{a}\right)\right]+\sum_{j} \frac{C}{2}\left(x_{j+1}-x_{j}-b\right)^{2} .
\end{aligned}
$$

Dicho modelo puede ser justificado en base a estudiar variables que cambian en una dimensión espacial, quedarnos con el primer término de la expansión en serie de Fourier del potencial sustrato y quedarnos en la aproximación armónica del desarrollo en serie de Taylor del potencial de interacción entre vecinos.

El hamiltoniano escrito anteriormente corresponde al modelo estándar del modelo. Variaciones del mismo incluyen partículas diferentes (cadena diatómica por ejemplo), términos adicionales en el potencial de interacción $W$, otros

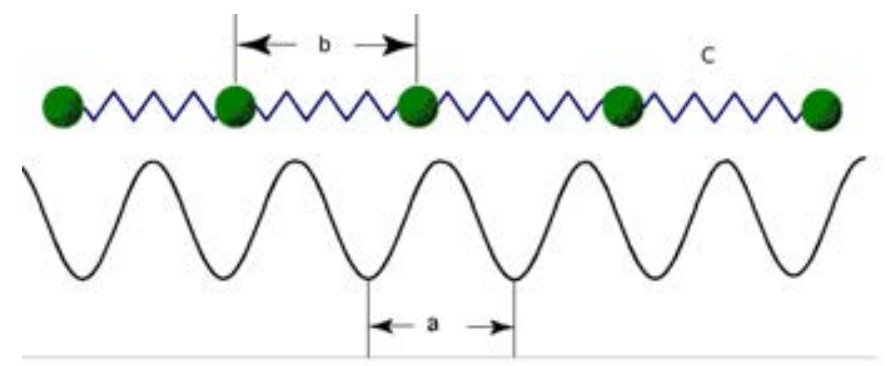

Figura 2.3: Esquema que representa el modelo Frenkel-Kontorova como una cadena de partículas conectadas a través de resortes armónicos de constante elástica $C$ y longitud de equilibrio $b$ sometida a un potencial sinusoidal externo de intensidad $V_{0}$ y periodo $a$. 


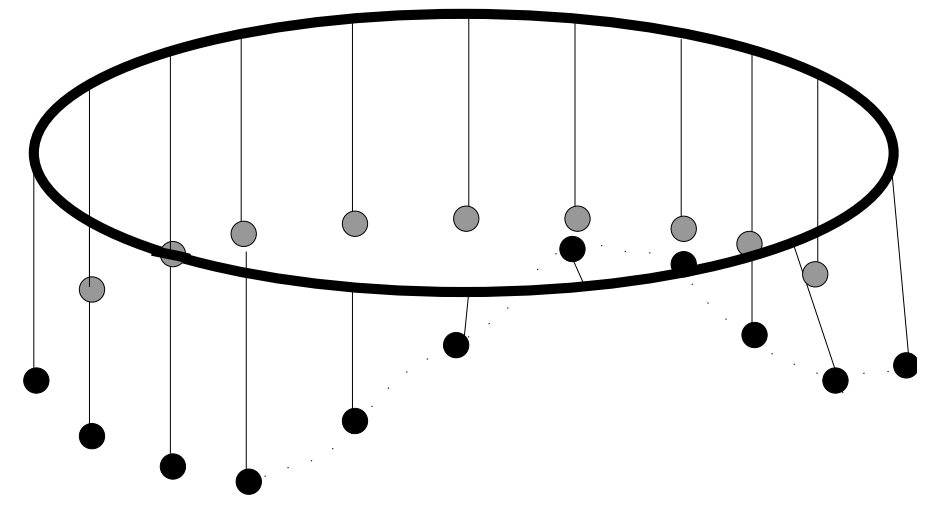

Figura 2.4: Arreglo de péndulos acoplados, equivalente a un arreglo en anillo de uniones Josephson con un solitón atrapado

harmónicos, desorden,... en el potencial substrato $V$ y extensión a dos o tres dimensiones.

La representación esquemática del modelo se dibuja en la figura 2.3 en donde las partículas o átomos se han dibujado en verde, acoplados por resortes armónicos y sometidos a un potencial sinusoidal de periodo $a$. Las propiedades del sistema están gobernadas por la competencia entre dos escalas de longitud (impuestas por $a$, la periodicidad del potencial sustrato, y $b$ la distancia de equilibrio entre vecinos) o dos escalas de energía (dadas por $V_{0}$ y $C$ ). Dicha competencia produce frustración en el sistema y un rico diagrama de fases, donde una vez fijado $a$ y $C$ por ejemplo, la densidad media de partículas en el estado fundamental en función de $b$ para distintos valores de la amplitud del potencial $V_{0}$ presenta estructura fractal (devil's stircase) [43, 57, 58].

Como modelo mecánico, el sistema es equivalente a una cadena de péndulos acoplados por muelles de torsión (figura 2.4). Como modelo de un sistema físico ha sido empleado para estudiar dislocaciones en sólidos, dinámica de átomos y capas de átomos adsorbidos en superficies de cristales, sistemas con fases conmensuradas e inconmensuradas, cadenas magnéticas, modelos no lineales de dinámica de $\mathrm{ADN}$, conductores superiónicos, modelos básicos de fricción de superficie y redes de uniones Josephson. [45]

Midiendo energía en unidades de $V_{0} / 2$, espacio en unidades de $a / 2 \pi$ y 
tiempo en unidades de $(a / 2 \pi) \sqrt{2 m / V_{0}}$ podemos reescribir

$$
\tilde{H}=\sum_{j}\left[\frac{1}{2}\left(\frac{d y_{j}}{d \tau}\right)^{2}+\left(1-\cos y_{j}\right)+\frac{\widetilde{C}}{2}\left(y_{j+1}-y_{j}-\widetilde{b}\right)^{2}\right]
$$

donde reconocemos una expresión similar a la que vimos para el anillo de uniones Josephson. Si además estamos interesados en la dinámica de la cadena bajo la acción de una fuerza externa, amortiguamiento y fluctuaciones térmicas recuperamos las mismas ecuaciones dinámicas. Por lo tanto, encontramos en el anillo de uniones Josephson un sistema experimental ideal para el estudio de las propiedades dinámicas de este modelo tan importante.

\subsubsection{La ecuación de sine-Gordon}

En el límite en el que el acoplamiento entre vecinos es grande, entonces la sucesión de posiciones de las partículas $x_{j}$ varía muy lentamente y el hamiltoniano del sistema pueden ser visto como la discretización de un campo espacial continuo $u(x), x_{j}=u(x=j)$. En este caso es posible derivar la versión continua del sistema y despreciando los efectos de discretitud de la cadena, se encuentra la siguiente ecuación para la dinámica hamiltoniana del campo $u$ :

$$
\frac{\partial^{2} u}{\partial t^{2}}-d^{2} \frac{\partial^{2} u}{\partial x^{2}}+\sin u=0
$$

Dicha ecuación tiene el nombre de ecuación de sine-Gordon. Debido a esta relación el modelo Frenkel-Kontorova también ha sido referido en numerosas ocasiones como modelo sine-Gordon discreto.

La ecuación de sine-Gordon [59] es una de las ecuaciones no lineales en el continuo más estudiadas. Originalmente fue introducida en el siglo XIX, pero sin embargo ha sido estudiada con gran interés a partir de los años 1970 debida a la presencia de solitones entre sus soluciones [59, 60, 61]. Una de sus propiedades más sobresalientes es que la ecuación es completamente integrable y tiene soluciones exacta tipo solitón, multi-solitón y breather.

Los solitones son debidos a la degeneración del estado fundamental del sistema. Un solitón (antisolitón) se corresponde con una solución exacta de la ecuación dada por:

$$
u(x, t)=4 \tan ^{-1}\left[\exp \left( \pm \frac{x-v t}{\sqrt{1-(v / c)^{2}}}\right)\right] .
$$

En este caso el campo $u(x, t)$ puede ser visto como una solución que conecta dos mínimos equivalentes cercanos y que se mueve sin alterar su perfil espacial a una velocidad $v$, con velocidad máxima $c$ (ver figura 2.5). 


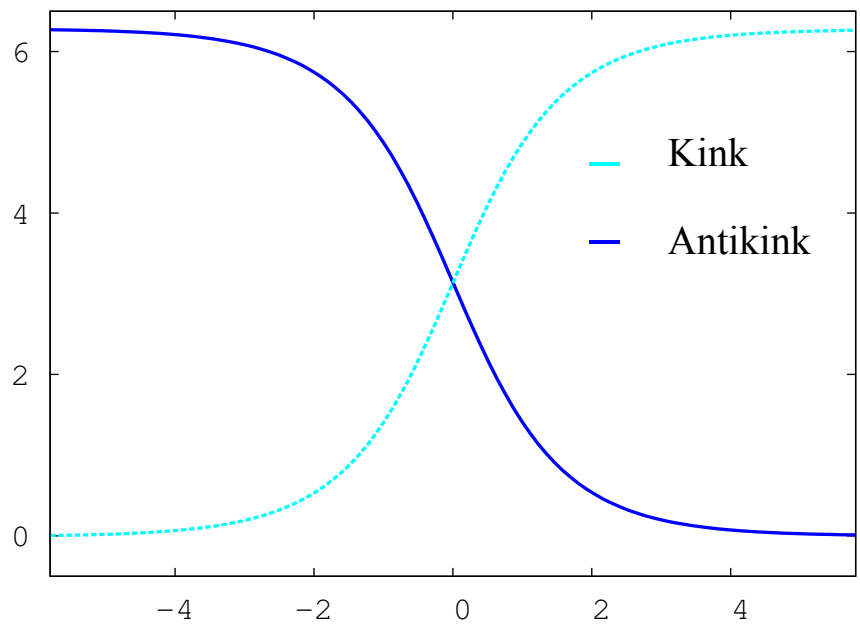

Figura 2.5: Solución de kink y antikink (solitón - antisolitón) de la ecuación de sineGordon

\subsubsection{Solitones en física no lineal}

En el capítulo anterior hemos introducido el concepto de solitón en el marco de la ecuación de sine-Gordon. Su característica esencial es la de poseer un perfil espacial localizado, el campo es constante salvo en una región estrecha donde cambia de manera continua de 0 a $\pm 2 \pi$, y moverse libremente a lo largo del espacio sin deslocalizarse.

Los solitones fueron descritos por primera vez por el ingeniero escocés J. Scott Russell en 1834. Rusell observó como un bote frenado bruscamente en un canal de agua generaba un "montículo" de agua que se alejaba del bote a gran velocidad manteniendo su forma y velocidad. Russell, montado a caballo, pudo seguir el montículo durante varios kilómetros. Este sorprendente fenómeno, hoy se conoce bajo el nombre de solitón.

Los solitones son un ejemplo de estructura coherentes que aparecen como solución de cierto tipo de ecuaciones no lineales (los vórtices y los breathers son otros ejemplos). Como hemos comentado anteriormente, un solitón es una onda solitaria que mantiene su forma mientras viaja a velocidad constante. En los solitones se pone de manifiesto un equilibrio entre un término dispersivo y otro no lineal de tendencia contraria. Los solitones surgen como soluciones de un amplio número de ecuaciones diferenciales no lineales, como la ecuación de Korteweg-de Vries, la ecuación de Schrödinger no lineal o la ecuación de sineGordon. En el caso de la ecuación de sine-Gordon, los solitones además son topológicos; esto es, son estables frente a perturbaciones que lo alejen del estado 
fundamental. Dicha robustez tiene su origen en las condiciones de frontera de los mismos, así en la ecuación $2.27 u(-\infty)=0$ y $u(+\infty)= \pm 2 \pi$.

Como sistema físico quizás los ejemplos más espectaculares de solitones se corresponde con los casos de solitones en fibras ópticas y en redes largas de uniones Josephson. En el primer caso los solitones pueden ser usados para la trasmisión de información en fibras. En el segundo se corresponde con la existencia de cuantos de flujo magnético o fluxones, que también pueden ser transportados a lo largo de la unión superconductora.

Dos excelentes referencias sobre las propiedades de los solitones son el libro de Remoissenet [60] y el de Dauxois y Peyrard [61].

\subsubsection{Solitones en el anillo de uniones Josephson}

En las secciones anteriores hemos visto como un anillo de uniones Josephson puede ser considerado como una versión discreta de una unión Josephson larga o una ecuación de sine-Gordon. Por ello, los fluxones o cuantos de flujo magnético atrapados en el anillo se corresponden con la versión discreta de los solitones de las ecuaciones continuas. Existen diversos nombres que se refieren a este mismo objeto. Así, en el contexto de la red de uniones Josephson nos referimos a fluxones, en el caso de sistemas de fases moduladas se ha acuñado el término de disconmensuraciones, y en el caso general se utiliza el concepto de solitón, solitón discreto o kink.

Tal y como hemos anunciado en otras secciones el objeto de este trabajo de tesis doctoral ha sido el estudio de algunas de las propiedades de los fluxones atrapados en anillos de uniones Josephson, fundamentalmente el estudio de como las curvas de desanclaje están afectadas por la temperatura. El capítulo 3 de la tesis lo vamos a dedicar a presentar algunas de las propiedades más sobresalientes de los fluxones en anillos de uniones Josephson, no obstante nos parece adecuado en este momento avanzar y resumir algunas de ellas.

Hemos visto que las ecuaciones para la dinámica de un anillo de uniones Josephson (o una red de uniones Josephson acopladas en paralelo) corresponden a las ecuaciones de la dinámica del modelo Frenkel-Kontorova o la ecuación de sine-Gordon discreta. Trabajos de revisión de estos sistemas son [43] y [45].

En el caso de las redes que hemos denominado homogéneas (formadas por celdas de igual área y bajo la acción de campos magnéticos externos uniformes) las ecuaciones que describen la dinámica de un anillo de uniones Josephson están dadas por:

$$
h_{j}\left(\ddot{\varphi}_{j}+\Gamma \dot{\varphi}_{j}+\sin \varphi_{j}+\tilde{i}_{j}\right)=\lambda\left(\varphi_{j+1}-2 \varphi_{j}+\varphi_{j-1}\right)+i_{\mathrm{ext}},
$$

con $j=1, \ldots N$ y condiciones de contorno $\varphi_{N+1}=\varphi_{1}+2 \pi n_{k}$ y $\varphi_{0}=\varphi_{N}-2 \pi n_{k}$ 


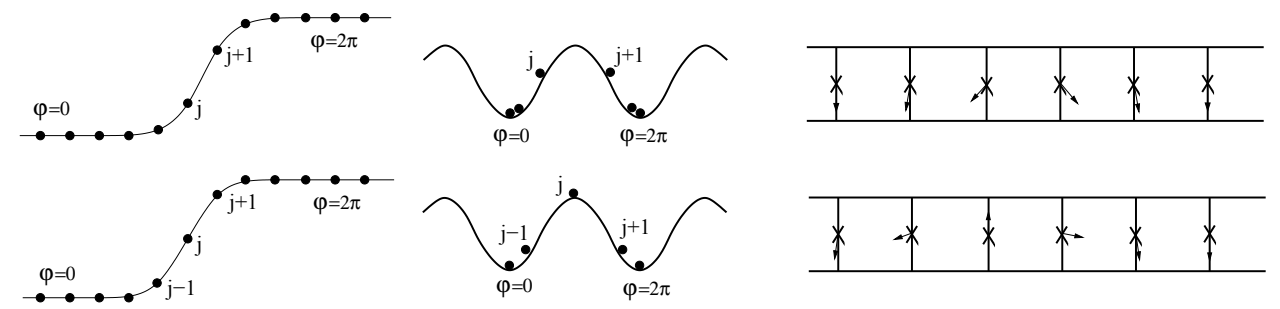

Figura 2.6: Tres representaciones diferentes de las configuraciones de energía mínima (arriba) y de máximo local (abajo) de un fluxón. Fases (izquierda), representación de energía potencial (centro) y representación angular (derecha).

donde $n_{k}$ es el número de kinks o fluxones atrapados en el anillo. Los kinks (o anti-kinks) son configuraciones de las fases en las que éstas van de 0 a $2 \pi(-2 \pi)$ a lo largo de la red y como vimos en su momento se corresponden a un cuanto de flujo magnético o fluxón atrapado en el sistema.

La existencia de kinks no depende crucialmente de la discretitud del sistema, y son soluciones en muchos aspectos similares a los solitones de la versión continua de la ecuación. Sin embargo, muchas de las propiedades de los kinks en sistemas discretos dependen crucialmente del carácter discreto de los mismos. La existencia de una red discreta destruye la invarianza traslacional presente en los modelos continuos. En la red, el kink es invariante sólo bajo traslaciones discretas a lo largo de la red y, debido a esta discretitud, aparece un potencial de anclaje o potencial de pinning a la red; esto es, existe una energía mínima que hay superar para moverse a lo largo de la red (Fig. 2.6). Esa barrera de energía es lo que se conoce como barrera de Peierls-Nabarro y decrece rápidamente $\operatorname{con} \lambda$.

Podemos ir un poco más lejos y definir, no sólo una barrera de PeierlsNabarro, sino también un potencial de Peierls-Nabarro $V_{P N}$ para el kink. Dicho potencial representa el perfil de energía cuando movemos un kink desde una posición de mínimo a otra equivalente. Como veremos, si identificamos la posición del kink por la coordenada de su centro de masas, $X_{C M}$, en el caso de una red regular $\left(h_{j}=1\right)$ el perfil de energía potencial $E\left(X_{C M}\right)$ es muy aproximadamente sinusoidal.

$$
V_{P N}(X) \simeq \frac{E_{P N}}{2}(1-\cos X) .
$$

En el caso de redes irregulares, el potencial efectivo no es puramente sinusoidal y, hasta cierto punto, puede ser diseñado a voluntad jugando con el valor de los acoplamientos $\lambda_{j}$ y la sucesión de valores de $h_{j}$.

Una vez introducido el potencial de Peierls-Nabarro del sistema, podemos escribir la ecuación efectiva de la dinámica del centro de masas del kink que, en 
la presencia de corrientes externas, amortiguamiento y fluctuaciones térmicas y en primer orden de aproximación se corresponde con:

$$
m \ddot{X}+m \Gamma \dot{X}+\frac{E_{P N}}{2} \sin X=i+\xi(t),
$$

donde $m$ es la masa efectiva del kink

Esta imagen de un kink o fluxón como una partícula independiente es muy útil para entender muchas de las propiedades dinámicas del mismo. No obstante, como veremos, en algunos casos es necesario tener en cuenta el resto de grados de libertad del sistema. Así por ejemplo cuando el fluxón se mueve en el anillo excita los modos de baja amplitud del sistema. Esta excitación es muy importante en el caso de redes con amortiguamiento pequeño en donde es posible identificar fenómenos de resonancia entre la frecuencia asociada al movimiento del fluxón en la red y las frecuencias de los modos de pequeña amplitud del sistema [41, 42].

\subsection{Efecto Ratchet en ciencia no-lineal}

Bajo el nombre de motores brownianos se conocen una serie de sistemas que operan de manera eficiente en sistemas fuera del equilibrio para obtener movimiento direccional bajo la acción de fuerzas cuyo promedio es nulo. Muchos de estos motores están basados en la explotación de lo que se conoce como efecto ratchet, es decir, explotan la existencia de potenciales espacialmente asimétricos. En particular estos dispositivos se caracterizan básicamente por poder rectificar las fluctuaciones térmicas y poseer alguna simetría espacial o dinámica rota. El campo de los motores brownianos ha generado gran curiosidad y trabajo a lo largo de los últimos años [62,63]. Dos excelentes artículos de revisión del tema son los trabajos de Reimann [64] y de Hänggi y Marchesoni [65].

A escala microscópica, la dinámica de muchos sistemas está regida por la fluctuaciones. El movimiento de las partículas de estas dimensiones, puede compararse al de un objeto macroscópico caminando a través de un huracán: las fuerzas que impulsan la partícula a lo largo de su camino eventualmente son insignificantes en comparación con las fuerzas aleatorias ejercidas por el ambiente. Aún así, como ocurre en el caso de las células vivas, se estructuran bombas de iones, se construyen proteínas, y los motores moleculares son capaces de transportar partículas de una zona o otra del interior celular [66, 67]. Los sistemas y mecanismos responsables de realizar las tareas deseadas en este ambiente dominado por las fluctuaciones se denominan motores Brownianos.

Como hemos dicho anteriormente, estos dispositivos están caracterizados por la ausencia de alguna simetría. Sin ánimo de dar una clasificación exhaus- 
tiva podemos distinguir entre:

i) Rectificadores de señales simétricas. Siguiendo el trabajo de Hänggi y Marchesoni [68] podemos decir que una condición necesaria para la rectificación de señales simétricas, ya sean aleatorias o periódicas en el tiempo, es la ausencia de simetría espacial en el potencial sustrato efectivo que ve la partícula sometida a dichas señales. Dicha asimetría en el potencial ha llevado a acuñar estos dispositivos con el nombre de ratchets, en recuerdo del trabajo de Feymann sobre the ratchet and pawl en sus célebres lecciones de física $[69,70]$. Un sistema de rueda dentada y trinquete es incapaz de rectificar las fluctuaciones térmicas del equilibrio, en acuerdo con el segundo principio de la termodinámica. Sin embargo, esto no es así para el caso de sistemas fuera del equilibrio. Entre estos sistemas se incluyen los denominados rocking ratchets y flashing ratchets.

ii) Rectificación debida a señales asimétricas. Si el potencial efectivo es simétrico bajo reflexión la única manera de introducir un movimiento direccional de partículas brownianas consiste en someterlas a fuerzas asimétricas, ya sean éstas deterministas o aleatorias. El caso más sencillo es el de rectificación bajo la acción de una señal biarmónica con frecuencias conmensurables, mezcla armónica [71, 72, 73]. También es posible obtener rectificación en el movimiento Browniano de partículas sometidas a fuerzas simétricas. Para ellos es necesario modular la amplitud del potencial sustrato, el cual es espacialmente simétrico. El efecto producido se denomina Gating Ratchet y puede generar una corriente dirigida de partículas con la disminución de las barreras de potencial sincronizadas con el movimiento producido por la fuerza aditiva [74, 75].

iii) Rectificación debida a efectos colectivos. La introducción de interacción entre las partículas que forman el sistema puede dar lugar a una gran riqueza de resultados y fenomenología, entre ellos la rectificación de señales simétricas en el caso de potenciales también simétricos. En sistemas superconductores que transportan vórtices interactuantes, por ejemplo, aparece el efecto ratchet como consecuencia de las interacciones de largo alcance entre los vórtices. Los vórtices anclados crean un perfil de densidad de flujo asimétrico, el cual resulta en un potencial efectivo asimétrico para los vórtices intersticiales no anclados [76, 77, 78]. El interés de los dispositivos ratchet de vórtices, está en que puede manifestar mecanismos colectivos por los que se produce la rectificación y además contribuir a la comprensión de los vórtices en superconductividad. Muchos motores biológicos, tales como, kinesina y miosina trabajan de modo colectivo. Los modelos teóricos han demostrado que la interacción entre los motores pueden dar lugar a fenómenos complejos como el desplazamiento bidi- 
reccional, oscilaciones, histéresis y la formación de estructuras dinámicas. la formación del transporte intracelular, la motilidad celular, muscular y oscilaciones durante la mitosis, son algunos ejemplos que pueden ser descritos por dichas teorías. El comportamiento cooperativo no es exclusivo de los motores moleculares, también las proteínas pueden interactuar y reflejar cambios conformacionales y transiciones de fase de no equilibrio. Las teorías que describen, respectivamente, el comportamiento colectivo de motores moleculares y la fricción en sólidos presentan analogías. En ambos casos, las partículas saltan entre pozos de potencial consecutivos y disipan la energía elástica durante los ciclos de anclaje y desanclaje [79].

\subsubsection{Rocking ratchet}

Consideremos la dinámica de una partícula sometida a fuerzas deterministas $F(t)$ periódicas, simétricas con $\langle F(t)\rangle=0$ y fuerzas estocásticas $\xi(t)$ de promedio nulo y no correlacionadas, $\langle\xi(t)\rangle=0 \mathrm{y}\langle\xi(t) \xi(t+\tau)\rangle \propto \delta(\tau)$. El amortiguamiento efectivo de la partícula está dado por $\eta$ y sea $V(x)$ un potencial efectivo periódico. Entonces,

$$
\ddot{x}(t)+\eta \dot{x}(t)+V^{\prime}(x)=F(t)+\xi(t) .
$$

En este caso para que la partícula muestre movimiento direccional, $\langle\dot{x}\rangle \neq 0$, necesitamos que el potencial sea asimétrico. El modelo arquetipo de potencial asimétrico unidimensional es un potencial lineal a trozos [80] o el potencial propuesto por Bartussek y colaboradores [81] (ver fig. 2.7):

$$
V(x)=-V_{0}[\sin (2 \pi x / L)+0.25 \sin (4 \pi x / L)] .
$$

El sistema descrito anteriormente es conocido como rocking ratchet. Resulta fácil ver que para ciertos valores de los parámetros el sistema presenta movimiento direccional dado que la fuerza necesaria para mover la partícula es menor en una dirección que en la otra. La figura 2.8 esquematiza el proceso. La figura intermedia muestra una colección de partículas situadas en torno a los mínimos del potencial a $F=0$. Para ciertos valores de $F$ (negativos en este caso) las partículas permanece atrapadas en los mínimos del potencial (figura superior). Sin embargo, para ese mismo valor de $F$ pero positivo las barreras han desaparecido y las partículas se mueven a lo largo del potencial (figura inferior). Un potencial periódico cuyo módulo este dado por valores de $F$ como los mostrados en la figura presenta un movimiento direccional de las partículas. Este cuadro se ve modificado ligeramente por los diferentes valores adicionales del sistema (amortiguamiento, temperatura y frecuencia fundamentalmente), pero la imagen cualitativa general permanece. 


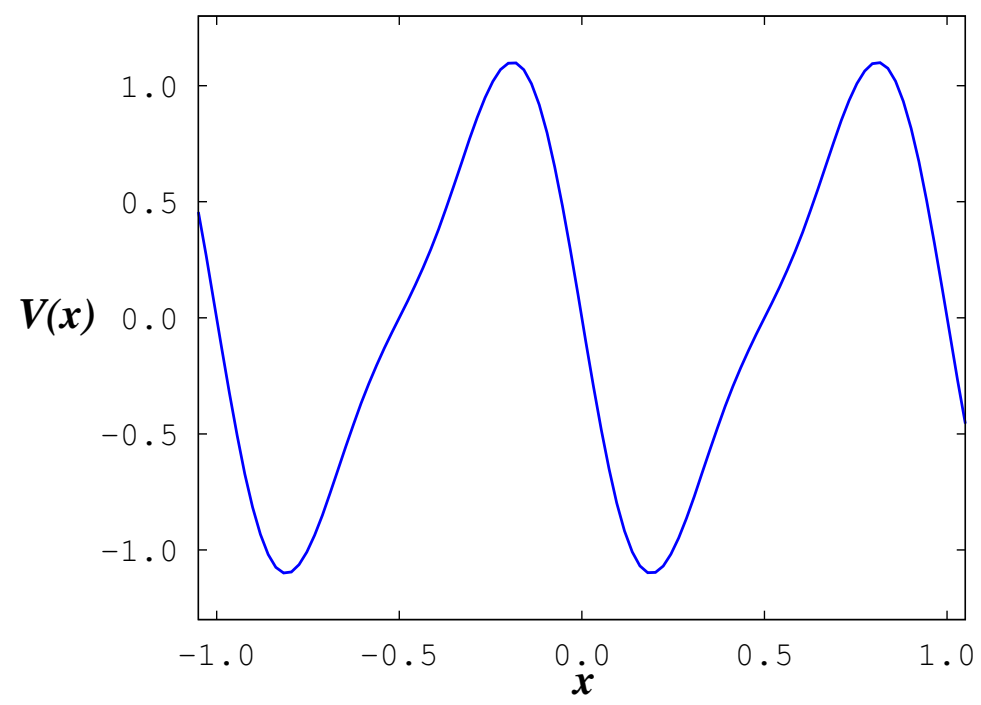

Figura 2.7: Ejemplo de potencial periódico asimétrico, potencial ratchet.

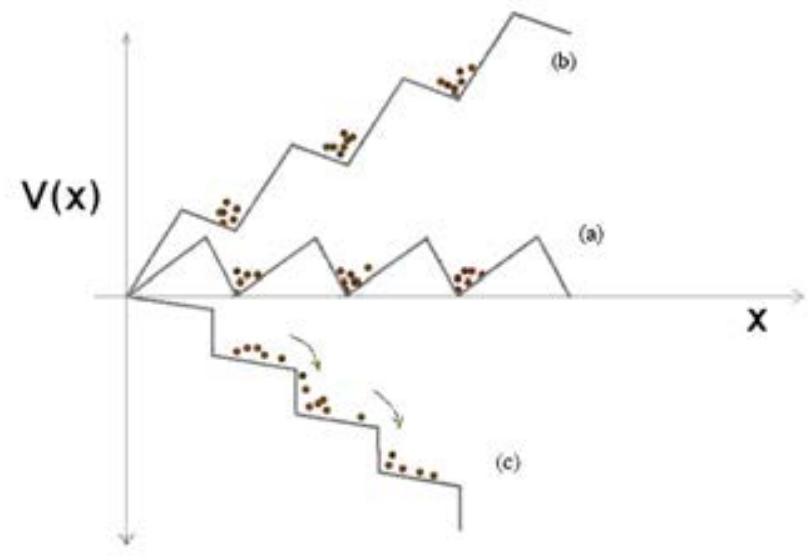

Figura 2.8: Esquema del funcionamiento de un rocking ratchet, donde se aplica una fuerza externa que varía en el tiempo de forma periódica

\subsubsection{Flashing ratchet}

Una variación interesante al caso anterior es el del flashing ratchet o ratchet pulsante. En un flashing ratchet $F(t)=0$ pero ahora el potencial asimétrico $V(x, t)$ depende explícitamente del tiempo de modo que su amplitud alterna su valor de 0 a $V_{0}$ periódicamente. Por lo general dichos sistemas se describen por una ecuación de Langevin, 


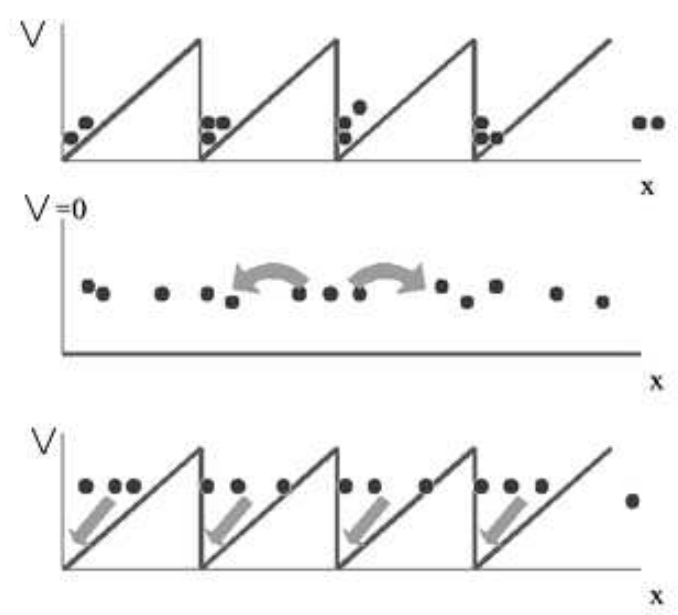

Figura 2.9: Esquema del funcionamiento de una flashing ratchet: las partículas Brownianas son atrapadas en un potencial periódico y asimétrico que puede estar prendido o apagado.

$$
m \ddot{x}=-\frac{\partial V(x, t)}{\partial x}-\gamma \dot{x}+\eta(t)
$$

En la ecuación (2.33) $m$ es la masa de la partícula, $\gamma$ es la disipación en el baño de calor y $\eta(t)$ es el ruido debido al baño de calor. Este ruido usualmente se toma como un ruido blanco Gaussiano que satisface la condición $\langle\eta(t)\rangle=0$ $\mathrm{y}\left\langle\eta(t) \eta\left(t^{\prime}\right)\right\rangle=2 k_{B} T \gamma \delta\left(t-t^{\prime}\right)$. En un flashing ratchet el potencial es prendido y apagado periódicamente (Fig. 2.9). En ausencia de potencial $V(x, t)$ las partículas se mueven por difusión. En este momento muchas partículas se acercan al mínimo del potencial por la izquierda mientras que pocas partículas han alcanzado el pico de la derecha (más lejano). Cuando el potencial es prendido, las partículas sobre la izquierda se deslizarán por la pendiente al próximo mínimo mientras que aquellas que estaban a la derecha, retornarán al pozo inicial. Por este motivo, obtenemos un movimiento neto a la izquierda que necesariamente requiere de la difusión para obtener un movimiento dirigido [65, 82].

\subsection{Dispositivos ratchet para fluxones}

En los últimos años el estudio del movimiento direccional de partículas ha cobrado relevancia no solo en el campo de los motores biomoleculares, sino también, en el campo de la nano y microtecnología. En esta línea y en el caso 

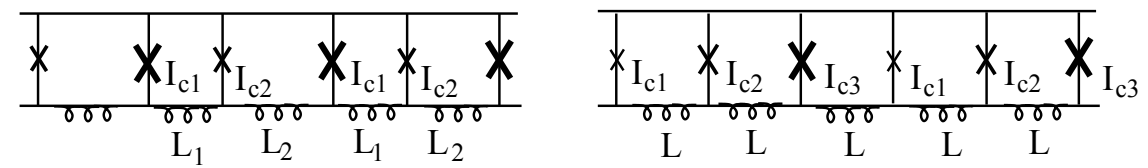

Figura 2.10: Dos geometrías de arreglos de uniones que producen un potencial efectivo asimétrico para el fluxón: alternancia de uniones de dos corrientes críticas y celdas de dos tamaños (izquierda) y alternacia de uniones de tres corrientes críticas con celdas iguales (derecha).

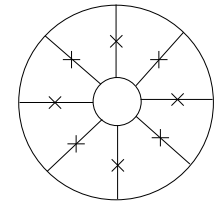

(a)

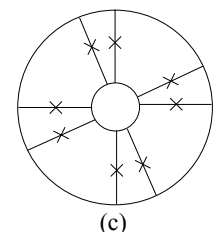

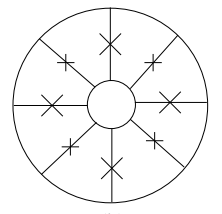

(b)

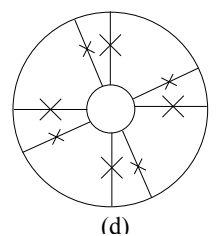

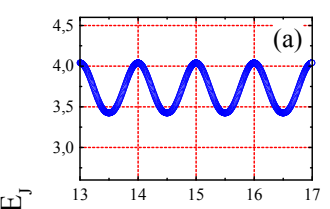

空

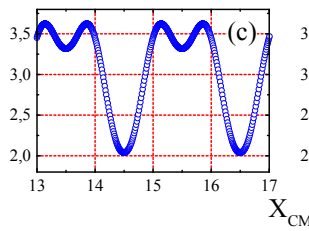

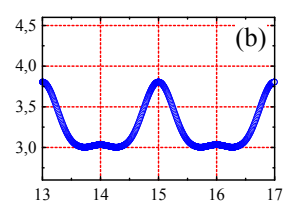

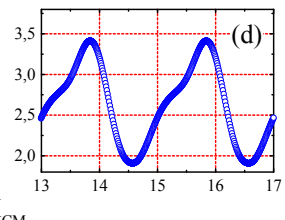

Figura 2.11: Cuatro arreglos diferentes: (a) Anillo regular. (b) Anillo alternando corrientes críticas. (c) Anillo alternando área de las celdas. (d) Anillo ratchet alternando corrientes críticas y área de celdas. La figura de la derecha muestra los correspondientes perfiles de energía $E\left(X_{C M}\right)$. Únicamente la configuración (d) genera el perfil ratchet.

de dispositivos superconductores, resulta de interés natural el diseño y estudio de sistemas que permitan el movimiento direccional de vórtices y fluxones en sistemas superconductores [76, 77, 78] en general y en redes de uniones Josephson en particular $[83,84,85]$, conformando lo que hemos llamado dispositivos ratchet para fluxones.

Ya hemos dicho que en una red de uniones Josephson un fluxón se comporta aproximadamente como una partícula que experimenta un potencial periódico, marcado por la periodicidad espacial de la red. En el caso de una red regular (todas las uniones y celdas son iguales) la simetría en el sistema es total y en ausencia de corrientes no equilibradas no existe movimiento direccional de fluxones. Sin embargo, la flexibilidad del sistema nos permite combinar uniones y celdas de tamaños diferentes, lo que como veremos posibilita el diseño de dispositivos con potenciales substrato periódicos asimétricos [83, 84, 85].

Un potencial ratchet para el fluxón es obtenido utilizando una combinación adecuada de uniones y tamaños de celda (esto es autoinducciones). Los dos casos más simples (ver figura 2.10) corresponden a alternar uniones de dos 


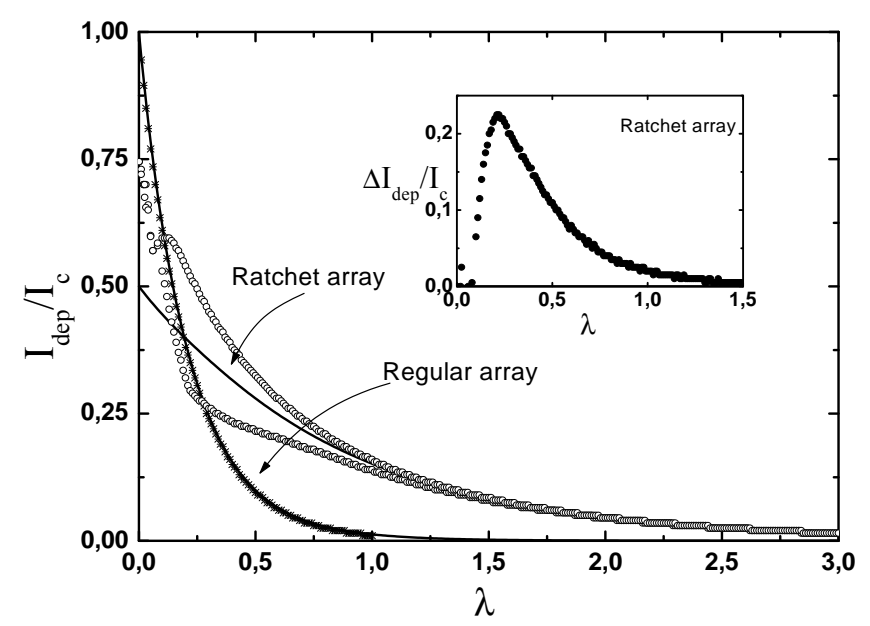

Figura 2.12: Corriente de desanclaje del Fluxon $I_{d e p}$ como función de $\lambda$ para los arreglos regular y ratchet. Las lineas sólidas representan las predicciones para los valores de $E_{P N}$ y los símbolos representa el cálculo numérico de las corrientes de desanclaje [85]. El recuadro muestra la diferencia $\left(\Delta I_{\text {dep }}\right)$ entre los valores absolutos de las dos corrientes de desanclaje para el arreglo ratchet.

corrientes críticas distintas y celdas de dos áreas; o a alternar uniones con tres corrientes críticas distintas (en este caso todas las celdas tienen el mismo área).

Tomemos como referencia de momento el primero de los dos casos descritos anteriormente. La figura 2.11 muestra cuatro redes diferentes y los potenciales efectivos para el fluxón en cada uno de los casos. Como esperamos, sólo la red (d) muestra un potencial ratchet para el fluxón. Tales redes se han construido y estudiado experimentalmente [84].

La figura 2.12 muestra la dependencia con $\lambda$ de la corriente de depinning positiva y negativa del fluxón (la corriente mínima necesaria para mover el fluxón) para el caso de la red regular y las ratchets[85]. Vemos como en el caso de la red ratchet $I_{d e p}^{+}$y $I_{d e p}^{-}$son significativamente diferentes para valores de $\lambda$ entre 0.1 y 0.9 . El inset muestra la diferencia $\left(\Delta I_{d e p}=I_{d e p}^{-}-I_{d e p}^{+}\right)$entre los valores de las dos corrientes para la red ratchet. Se ve que existe un moderado rango de valores de $\lambda$ para el cual un importante comportamiento asimétrico es esperado. El máximo de la curva se obtiene para $\lambda \sim 0.2$.

Viendo las ecuaciones que describen la dinámica de la red [Eq. (2.8) y siguientes], es fácil darse cuenta de que resulta mucho más conveniente estudiar redes formadas por celdas iguales, alimentada uniformemente y en ausencia de campos externos, ecuación (2.12). Este es el caso de la configuración con uniones de tres tamaños diferentes. Esta es la configuración que se usará a lo largo del capítulo 5 de la tesis doctoral para estudiar el desanclaje térmico de 
los fluxones en redes asimétricas. Las propiedades de los fluxones en dichas redes serán introducidas en la última sección del capítulo siguiente. 



\section{Capítulo 3}

\section{Fluxón como una partícula}

En el capítulo anterior hemos visto que en muchos casos las propiedades de un fluxón en una red de uniones Josephson pueden ser estudiadas en términos de una partícula. En este capítulo vamos a abundar sobre el alcance, consecuencias y limitaciones de esta imagen.

\subsection{Redes regulares}

Una red regular es aquella en la que todas las uniones y celdas son idénticas. En ese caso, como hemos visto en el capítulo anterior, la energía potencial del sistema viene dada por

$$
E / E_{J}=\sum_{j}\left[\left(1-\cos \varphi_{j}\right)+\frac{\lambda}{2}\left(\varphi_{j+1}-\varphi_{j}\right)^{2}\right] .
$$

Con respecto a las ecuaciones para la dinámica del arreglo en presencia de corrientes externas y temperatura, tenemos

$$
\ddot{\varphi}_{j}+\Gamma \dot{\varphi}_{j}+\sin \varphi_{j}=\lambda\left(\varphi_{j+1}-2 \varphi_{j}+\varphi_{j-1}\right)+i_{\text {ext }}+\tilde{i}_{j}(t)
$$

donde $j=1, \ldots N$ y las condiciones de frontera para el anillo son $\varphi_{N+1}=$ $\varphi_{1}+2 \pi M$ y $\varphi_{0}=\varphi_{N}-2 \pi M$. El número entero $M$ es el número de fluxones o kinks atrapados en el anillo.

Un fluxón o antifluxón, figura 3.1, corresponde a una configuración $\{\varphi\}_{\text {fluxon }}$ de las fases del anillo para el cual la fase incrementa en $\pm 2 \pi$ en una vuelta alrededor del anillo $\left(\varphi_{j+N}=\varphi_{j} \pm 2 \pi\right)$. Como hemos descrito en el capítulo anterior, al estudiar la dinámica de un fluxón es muy útil usar la imagen de este objeto colectivo y extendido como una partícula. Está aproximación ha sido extensamente estudiada en el pasado [44, 45, 87, 88, 86]. 


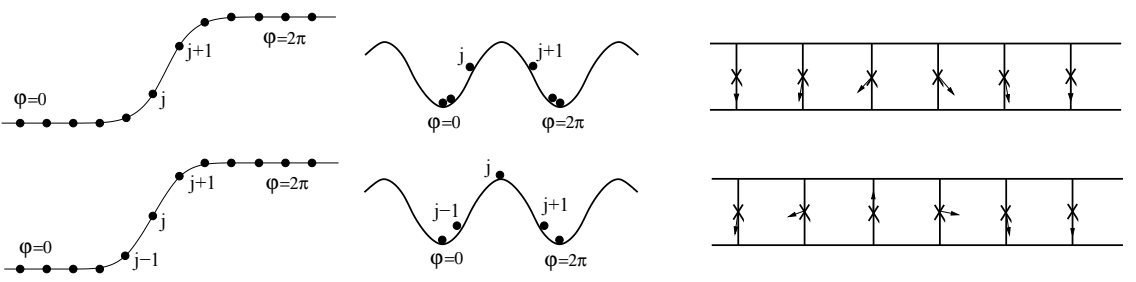

Figura 3.1: Tres representaciones diferentes de las configuraciones de energía mínima (arriba) y de máximo local (abajo) de un fluxón. Fases (izquierda), representación de energía potencial (centro) y representación angular (derecha).

Consideremos la nueva variable $X$, el centro de masas del fluxón definido por

$$
X=C \mp \sum_{j} \varphi_{j}
$$

El signo - corresponde al configuración de un fluxón, la fase se incrementa en $2 \pi$, mientras el signo + corresponde a un antifluxón. La constante $C$ la definiremos como $C=2 \pi(N+1 / 2)$ con $N$ el número de uniones en el anillo. Con esta definición la variable $\widetilde{X}=X / 2 \pi$ indica la posición del fluxón en la red.

En la aproximación más simple, la dinámica de un fluxón en un anillo, descrita por la ecuación (3.2), puede ser reducida a la dinámica de su centro de masas. A su vez, ésta es aproximada por la dinámica de una partícula masiva, forzada y amortiguada que experimenta un potencial sustrato periódico $V(X)$ (el potencial de Peierls-Nabarro).

$$
m(X) \ddot{X}+\eta m(X) \dot{X}+V^{\prime}(X)=F+\xi(\tau)
$$

Resaltamos los siguiente aspectos en esta aproximación:

- El origen del potencial de Peierls-Nabarro es el carácter discreto de la red. Por ello se trata de un potencial periódico cuya amplitud debe ir a cero cuando nos acercamos al límite continuo del sistema, $\lambda \rightarrow \infty$.

- $m(X)$ representa la masa efectiva del kink. Su origen es secillo cuando pensamos en término de la energía cinética del mismo: $\frac{1}{2} \sum_{j} \dot{\varphi}_{j}{ }^{2}=\frac{m}{2} \dot{X}^{2}$. Como consecuencia de las propiedades elásticas del fluxón, su masa efectiva depende de la posición del mismo, $m(X)$ : el fluxón no es un objeto rígido y su forma cambia cuando se mueve a lo largo de la red. 
- $F=i_{\text {ext }}$. El efecto de la fuerza externa es mover el fluxón y la fuerza externa efectiva que experimenta el fluxón es igual a la corriente por unión. De este modo la energía introducida en el sistema es la misma en las dos descripciones, $F X \equiv i_{\text {ext }} \sum_{j} \varphi_{j}$.

- Nos queda discutir el valor del amortiguamiento efectivo del sistema y el acoplo de éste al baño térmico. Este es un aspecto complejo. En su aproximación más sencilla la energía introducida en el sistema se disipa a través del movimiento del centro de masas y puede considerarse que $\eta=\Gamma \mathrm{y}$

$$
\langle\xi(\tau)\rangle=0 \quad y \quad\left\langle\xi(\tau) \xi\left(\tau^{\prime}\right)\right\rangle=2 m \Gamma k_{B} T \delta\left(\tau-\tau^{\prime}\right)
$$

Esta aproximación es equivalente a despreciar el efecto de los otros grados de libertad del fluxón y la disipación de energía a través de los cambios en el perfil del mismo, lo que conduce a una dependencia del damping efectivo con la posición del fluxón, $\eta(X)[87,88]$.

\subsubsection{La barrera y el potencial de Peierls-Nabarro}

Una de las propiedades fundamentales de los sistemas discretos es la falta de invariancia traslacional continua en los mismos. Dicha invariancia ha sido sustituida por una simetría discreta bajo traslaciones del centro de masas del fluxón un número entero de espaciados de red. Como resultado, no todas las posiciones espaciales son equivalentes y en el kink podemos distinguir entre la configuración de mínima energía, fluxón centrado entre dos sitios de la red, y una configuración de máximo local, fluxón centrado en un sitio de la red (ver la figura 3.1). La diferencia de energía entre estas dos configuraciones define la llamada barrera de Peierls-Nabarro del sistema y tiene el significado de barrera de energía que el fluxón debe sobrepasar para pasar de una configuración de mínima energía a otra equivalente. Dicha barrera es nula en el modelo continuo dado que en este caso todos las posiciones del kink son equivalentes (simetría continua bajo traslación espacial). Como consecuencia, en el sistema discreto el kink está anclado a la red, existe una fuerza de desanclaje o fuerza de depinning que es necesario aplicar para que el kink pueda moverse.

La figura 3.2 muestra la barrera de Peierls-Nabarro para un fluxón en función del parámetro de acoplamiento $\lambda$. En el límite $\lambda=0$ la configuración de fases para un fluxón en un mínimo sigue la secuencia $\{\ldots, 0,0,2 \pi, 2 \pi, \ldots\}$ mientras que la configuración de máximo local corresponde a $\{\ldots, 0,0, \pi, 2 \pi, 2 \pi, \ldots\}$. Ambas configuraciones tienen una diferencia de energía de $2 E_{J}$. Para valores pequeños de $\lambda$ estas configuraciones cambian ligeramente y no es difícil probar 


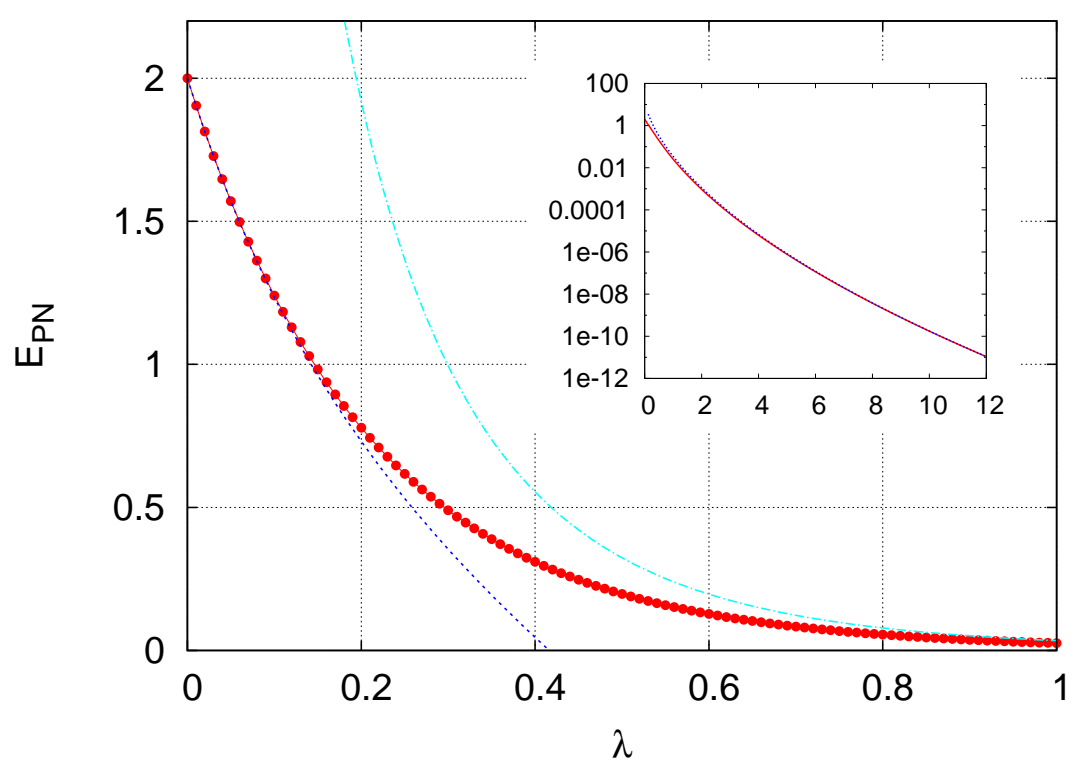

Figura 3.2: Evolución de la barrera de Peierls-Nabarro $E_{P N}$ frente a $\lambda$ calculada numéricamente y comparación con los resultados analíticos mostrados en el texto.

que en ese caso

$$
\frac{E_{P N}}{E_{J}} \simeq 2-\frac{\pi^{2} \lambda(1+\lambda)^{2}}{(1+2 \lambda)(1+3 \lambda)}
$$

En el límite opuesto, $\lambda \rightarrow \infty$, el fluxón se deslocaliza, su anchura diverge y la barrera se anula. Existen distintas estimaciones para el cálculo de la energía de Peierls-Nabarro en ese caso, ver por ejemplo Ref. [45]. De entre ellas encontramos que la siguiente es la que mejor se ajusta a nuestros resultados numéricos (figura 3.2):

$$
\frac{E_{P N}}{E_{J}}=\frac{16\left(1+2 \pi^{2} \lambda\right)}{\sinh \left(\pi^{2} \sqrt{\lambda}\right)}
$$

También es posible definir una energía potencial unidimensional que describe el potencial efectivo que el kink experimenta cuando pasa de una posición de mínimo a otra equivalente. Dicho potencial puede ser calculado aproximadamente a partir de la forma del solitón en el límite continuo del sistema [89, 45] obteniéndose

$$
V_{P N}(X) \simeq \sum_{l=0}^{\infty} B_{l} \cos (l X) \simeq V_{0}+\frac{E_{P N}}{2}(1-\cos X),
$$

con

$$
B_{l}=\frac{8 l\left(1+2 \pi^{2} \lambda l^{2}\right)}{\sinh \left(l \pi^{2} \sqrt{\lambda}\right)} E_{J}, \quad l \geq 1
$$




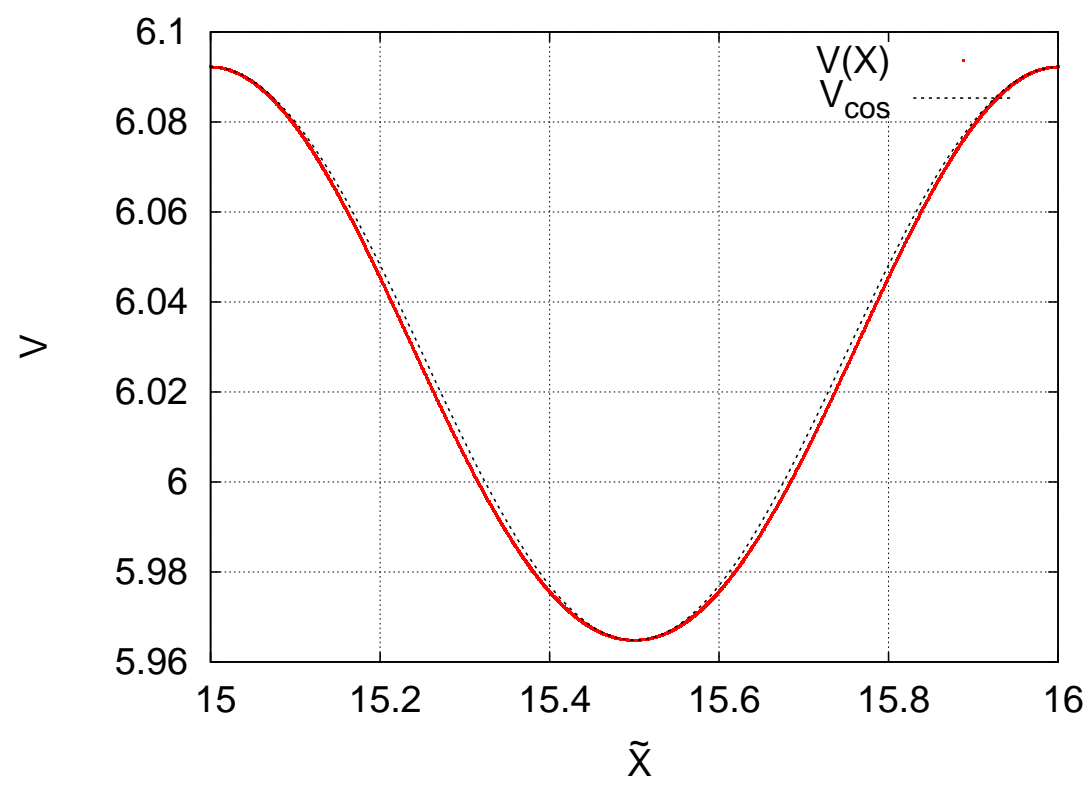

Figura 3.3: Potencial de Peierls-Nabarro, $V(\widetilde{X})$ para un fluxón en un anillo de treinta uniones para $\lambda=0.6$. La figura muestra en línea discontinua el perfil de un potencial sinusoidal de la misma amplitud y periodo.

El potencial puede ser calculado numéricamente para una red de tamaño arbitrario y con las condiciones de frontera que se deseen. Para ello basta con colocar un fluxón en una posición de máximo local y perturbarlo muy ligeramente para que caiga a uno de los mínimos cercanos. Si la trayectoria es sobreamortiguada, en cada momento la velocidad del fluxón es igual a la fuerza ejercida por el potencial; esto es, el gradiente del potencial, así que si calculamos la energía potencial del fluxón a lo largo de esa trayectoria tenemos el perfil de energías del potencial. La figura 3.3 muestra el potencial efectivo calculado de esa manera y lo compara con un potencial puramente sinusoidal. El cálculo ha sido realizado sobre una red con 30 uniones y para $\lambda=0.6$. En la figura vemos como la desviación respecto el perfil sinusoidal es muy pequeña, no obstante esa diferencia será relevante para calcular una de las magnitudes más importantes del sistema, la corriente de depinning del fluxón. Dicha corriente esta relacionada con la máxima pendiente del potencial, o el gradiente en el punto de inflexión. De hecho, cuando se estudien propiedades del fluxón relativamente cerca de esta corriente crítica es más eficiente definir el potencial de Peierls-Nabarro como

$$
V_{P N}(X) \simeq V_{0}+i_{\mathrm{dep}}(1-\cos X) .
$$

La figura 3.4 muestra la diferencia entre la corriente de depinning del fluxón calculada numéricamente $i_{\text {dep }} \mathrm{y}$ la correspondiente a un potencial sinusoidal 


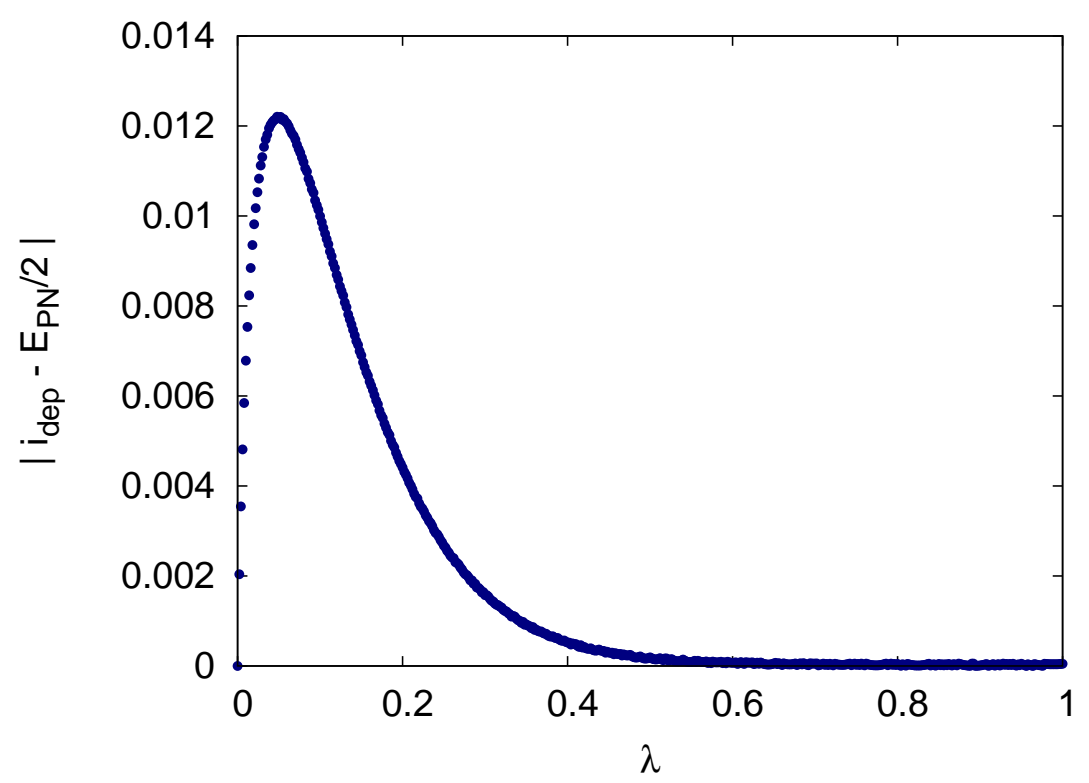

Figura 3.4: La figura muestra $\left|i_{\mathrm{dep}}-E_{P N} / 2\right|$ para ilustrar la diferencia entre la corriente de desanclaje del fluxón $i_{r m d e p}$ y la correspondiente a un potencial sinusoidal perfecto de barrera dada por $E_{P N}$. Ambas funciones han sido calculadas numériamente.

con la misma barrera de Peirls-Nabarro $E_{P N} / 2$. Podemos ver que esa diferencia es en general pequeña.

\subsubsection{Espectro de modos lineales}

Los modos lineales del sistema corresponden a las soluciones de pequeña amplitud y energía del mismo. Dichas soluciones pueden calcularse fácilmente si no hay fluxones atrapados en la red $(M=0)$. En la aproximación lineal y en ausencia de amortiguamiento y corriente externa, se tiene

$$
\ddot{\varphi}_{j}+\varphi_{j}=\lambda\left(\varphi_{j+1}-2 \varphi_{j}+\varphi_{j-1}\right)
$$

$j=1, \ldots N, \varphi_{N+1}=\varphi_{1} \mathrm{y} \varphi_{0}=\varphi_{N}$. Las soluciones de este sistema de ecuaciones tienen la forma: $\varphi_{j}(t) \sim \exp \left[i\left(\omega_{k} t-k j\right)\right]$ con espectro de frecuencias

$$
\omega_{k}^{2}=1+4 \lambda \sin ^{2}(k / 2) \quad(-\pi<k \leq \pi) .
$$

Esta relación de dispersión está caracterizada por la presencia de una banda finita con un gap dado por $\omega_{\min }=\omega_{0}=1$ y una frecuencia máxima dada por $\omega_{\max }=\omega_{\pi}=(1+4 \lambda)^{1 / 2}$. 


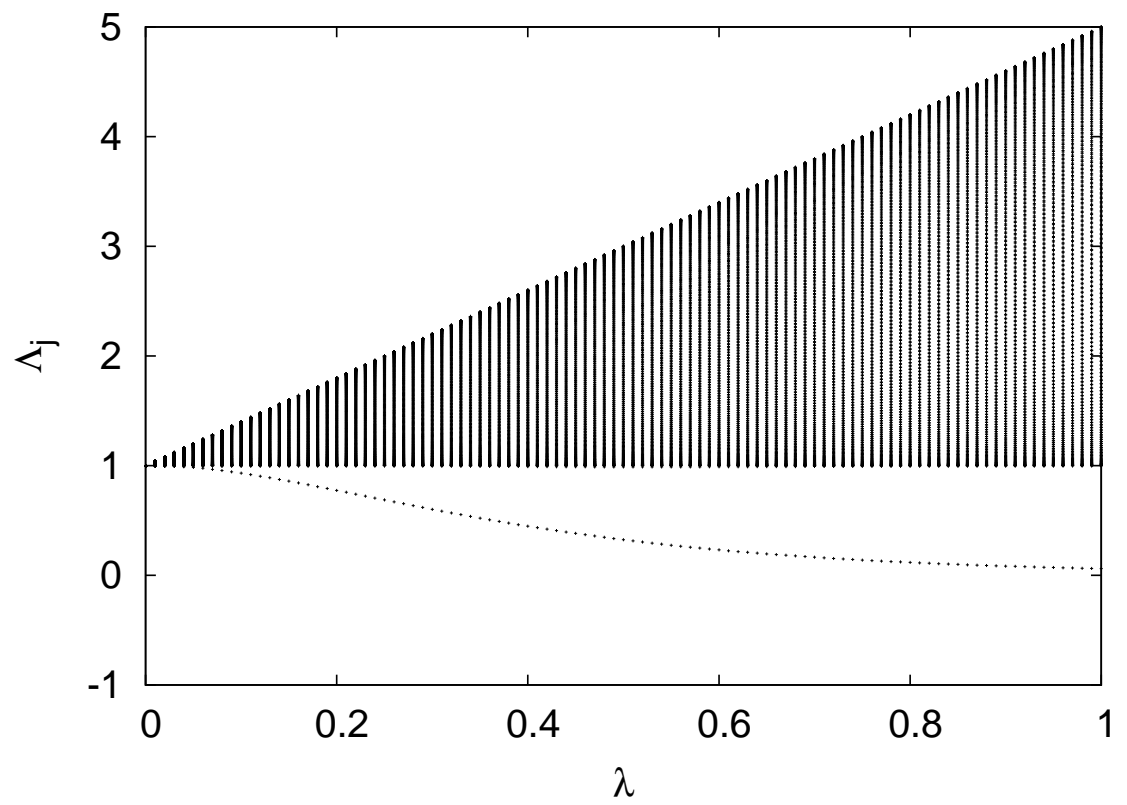

Figura 3.5: Autovalores de la matriz de estabilidad en el mínimo, $\Lambda_{j}=\omega_{j}^{2}$, para distintos valores del parámetro de acoplamiento $\lambda . N=300$.

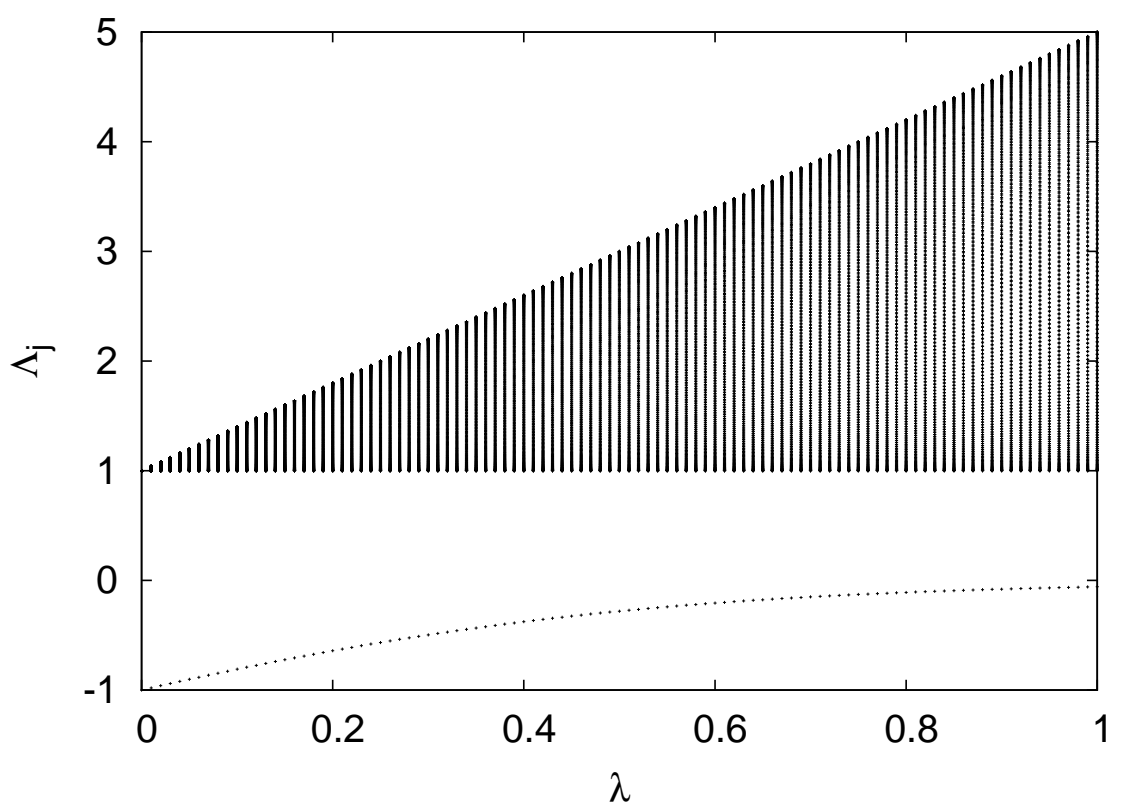

Figura 3.6: Autovalores de la matriz de estabilidad en el máximo local para distintos valores del parámetro de acoplamiento $\lambda . N=300$. 


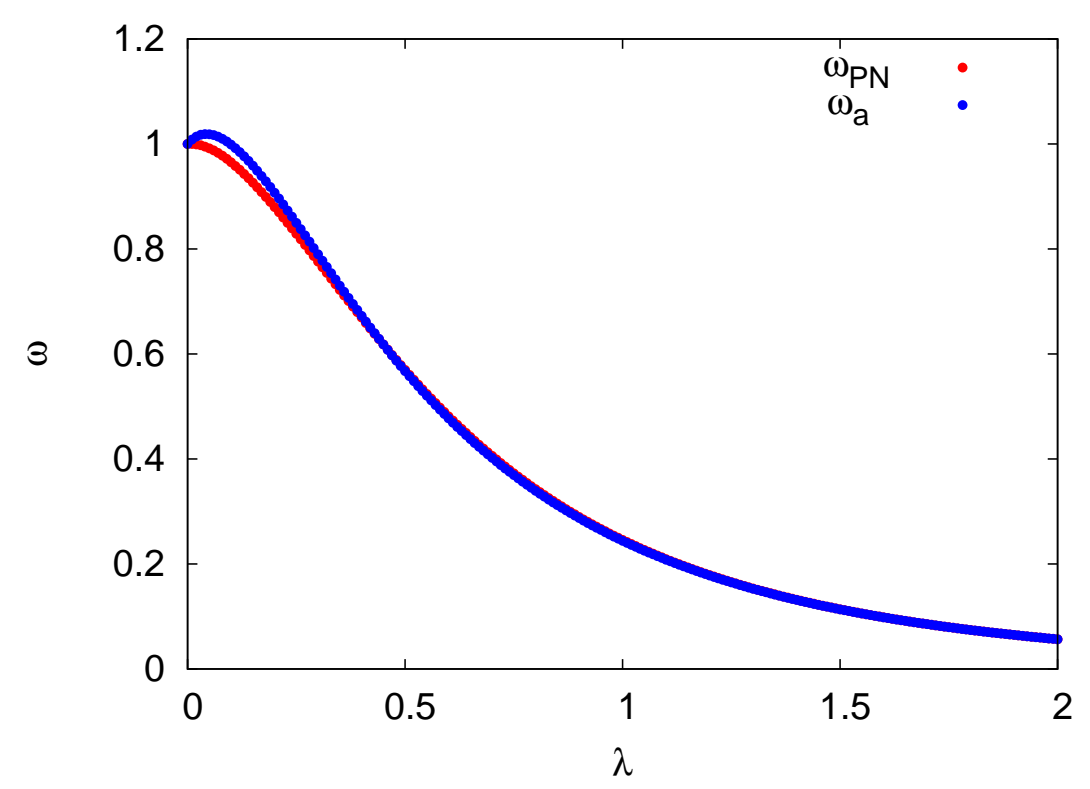

Figura 3.7: Frecuencia de Peierls-Nabarro (rojo), $\omega_{P N}$, y frecuencia de activación efectiva (azul), $\omega_{a}$, como función del parámetro de acoplamiento $\lambda . N=300$.

En presencia de un fluxón el espectro de modos cambia y ya no puede ser calculado analíticamente. En este caso para cada valor de acoplamiento $\lambda$ podemos calcular numéricamente la configuración de equilibrio $\{\varphi\}_{\text {fluxon }} \mathrm{y}$ linealizar las ecuaciones del sistema en torno a esta solución. La existencia del fluxón se plasma fundamentalmente en la aparición de un modo en el gap original. Dicho modo se corresponde con la frecuencia $\omega_{P N}$ de las oscilaciones de pequeña amplitud del fluxón en torno al mínimo del potencial efectivo $V_{P N}(X)$.

En la figura 3.5 se puede apreciar la banda de modos normales de frecuencias comprendidas entre 1 y $\sqrt{1+4 \lambda}$ y el modo adicional de frecuencia $\omega_{P N}$ igual a 1 para $\lambda \rightarrow 0$ y tendiendo a 0 para $\lambda \gg 1$.

También es fácil calcular la curvatura del potencial en torno al máximo local que define la posición de equilibrio inestable para el fluxón. En este caso se obtiene un autovalor negativo, asociado a la curvatura negativa en el máximo y $N-1$ positivos, ver figura 3.6. En los problemas de escape de un potencial multidimensional se define una frecuencia de activación efectiva $\omega_{a}$ dada por $\omega_{a}=\prod_{j=1}^{N} \omega_{j} / \prod_{j=1}^{N-1} \omega_{j}^{\prime}$, donde $\omega_{j}$ representa las $N$ frecuencias correspondientes a los modos normales en el mínimo y $\omega_{j}^{\prime}$ las frecuencias correspondientes a los $N-1$ modos estables del máximo. La figura 3.7 muestra la dependencia de $\omega_{a}$ con $\lambda$ y la compara con el valor de $\omega_{P N}$. 


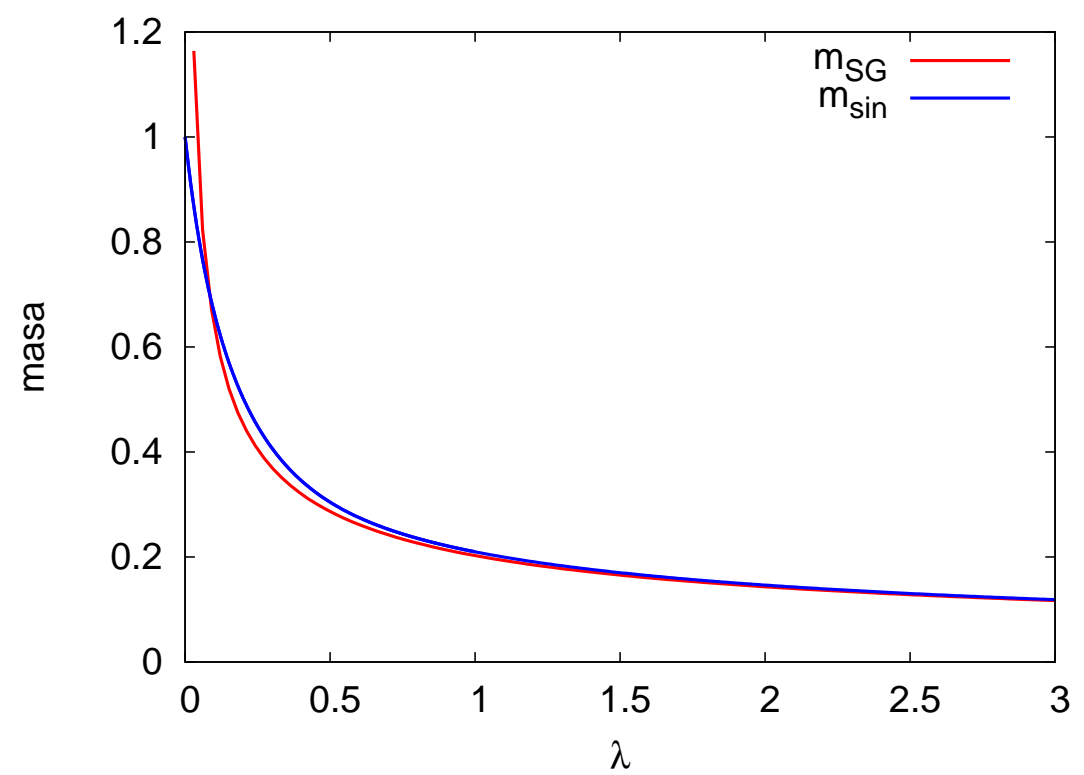

Figura 3.8: Masa efectiva del fluxón en función del acoplamiento $\lambda$ según la aproximación del continuo, $m_{S G}=2 /\left(\pi^{2} \sqrt{\lambda}\right)$ en rojo y según la aproximación de potencial sinusoidal $m_{\sin }$, en azul.

\subsubsection{La masa efectiva del fluxón}

Como hemos visto anteriormente en la ecuación efectiva para el fluxón aparece un término de masa, $m(X)$, que depende de la posición del mismo [45]. La dependencia espacial de la masa es fruto de la discretitud del sistema. Así, en principio podemos escribir

$$
m(X)=m_{S G}+\delta m(X)
$$

con $m_{S G}=2 /\left(\pi^{2} \sqrt{\lambda}\right)$ la masa efectiva del soliton en el continuo (ecuación de sine-Gordon) y $\delta m(X)$ la función periódica que incluye las correcciones por discretitud.

Existen dos aproximaciones al cálculo de la masa en reposo del fluxón (en el mínimo) a partir del conocimiento de la frecuencia de Peierls-Nabarro. La más sencilla asume la aproximación sinusoidal para el potencial de Peierls-Nabarro, entonces: $m_{\sin }=E_{P N} /\left(2 \omega_{P N}^{2}\right)$. En la segunda empleamos el valor determinado numéricamente para la curvatura en el mínimo $m_{\min }=V_{\min }^{\prime \prime} / \omega_{P N}^{2}$. Según estas definiciones, para $\lambda=0.6$ por ejemplo, $m_{S G}=0.262, m_{\sin }=0.275 \mathrm{y}$ $m_{\text {min }}=0.236$ [calculada tra aproximar $V(X) \simeq \frac{1}{2} 2.17\left(\frac{x-x_{0}}{2 \pi}\right)^{2}$. En la figura 3.8 se muestra el valor de la masa efectiva del fluxón para los distintos valores del acoplamiento $\lambda$ calculada según la aproximación del continuo (lo que he 


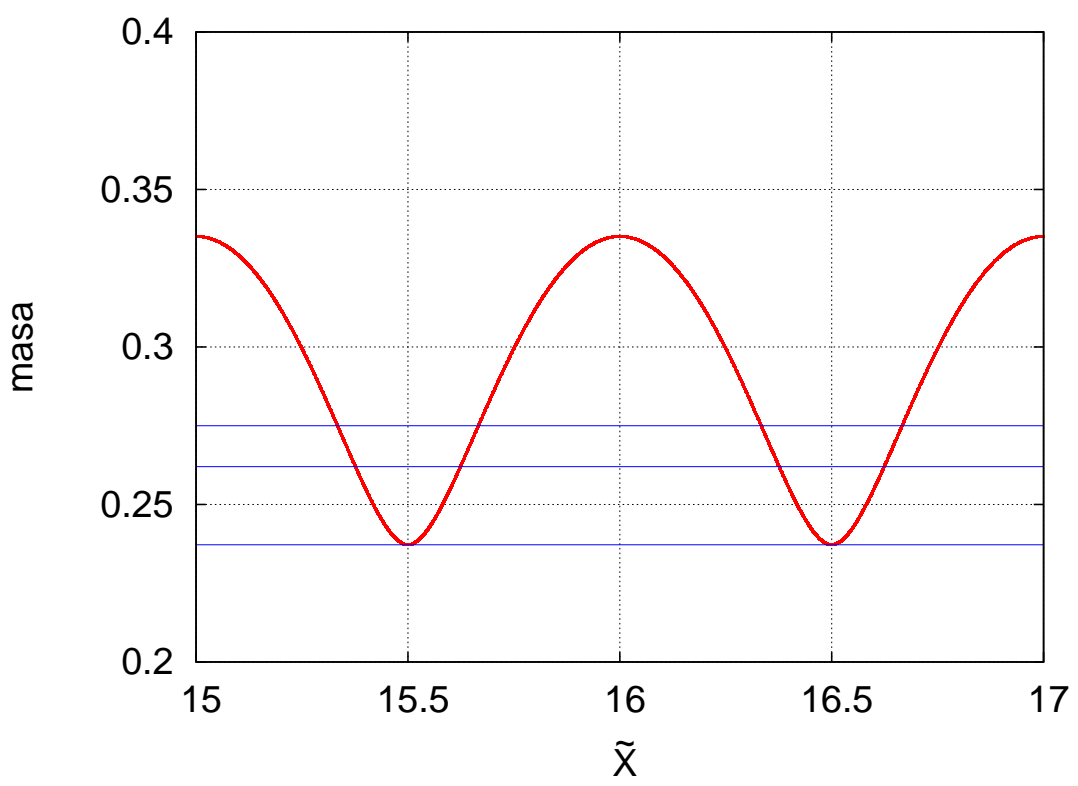

Figura 3.9: Calculo de la dependencia de la masa efectiva con la posición del centro de masas del fluxón $m(X)$, para $\lambda=0.6$. En azul se marca el valor según las tres aproximaciones discutidas en el texto $m_{\sin }=0.275, m_{S G}=0.262$ y $m_{\min }=0.236$.

llamado $m_{S G}$ ) y según la aproximación de potencial exactamente sinusoidal (lo que he llamado $m_{\text {sin }}$ ). Puede verse como la diferencia entre ambos valores es muy pequeña, salvo a valores muy pequeños de $\lambda$ donde la primera expresión diverge. En cualquier caso esta diferencia entra en la banda de variación espacial de la masa, $m(X)$. Así, por ejemplo, la figura 3.9 muestra el resultado de calcular $m(X)$ para el caso $\lambda=0.6$ y los tres valores de la masa anteriormente citados. Puede apreciarse como $m_{\text {min }}$ nos da el valor de la masa en el mínimo, pero las variaciones de la masa a lo largo del potencial son muy significativas, $m(X) \in(0.24,0.33)$.

Para el cálculo numérico de $m(X)$ hemos calculado la relajación en régimen sobreamortiguado del fluxón desde el máximo hasta el mínimo del potencial de Peierls-Nabarro, e igualado la energía cinética del sistema original con la del modelos reducido: $\frac{1}{2} \sum_{j} \dot{\varphi}_{j}^{2}=\frac{1}{2} m(X) \dot{X}^{2}$. También es posible obtener una expresión a partir de la forma de fluxón en el límite continuo del sistema [45]. Entonces

$$
m(X) \simeq m_{S G}+\sum_{l=1}^{\infty} A_{l} \cos (l X) \simeq
$$

con

$$
A_{l}=\frac{4 l}{\sinh \left(l \pi^{2} \sqrt{\lambda}\right)}
$$




\subsubsection{Un experimento numérico}

Para comprobar numéricamente algunas de las cuestiones estudiadas anteriormente, hemos estudiado las oscilaciones de relajación al equilibrio de un fluxón desde una condición de no equilibrio, cercana al mínimo, y su comparación con el resultado obtenido usando la teoría de partícula individual.

Para ello hemos elegido un anillo de $N=60$ uniones con $\lambda=0.6$. En este caso $E_{P N}=0.12739, \omega_{P N}^{2}=0.23165$ y $m=0.236$. En la aproximación de
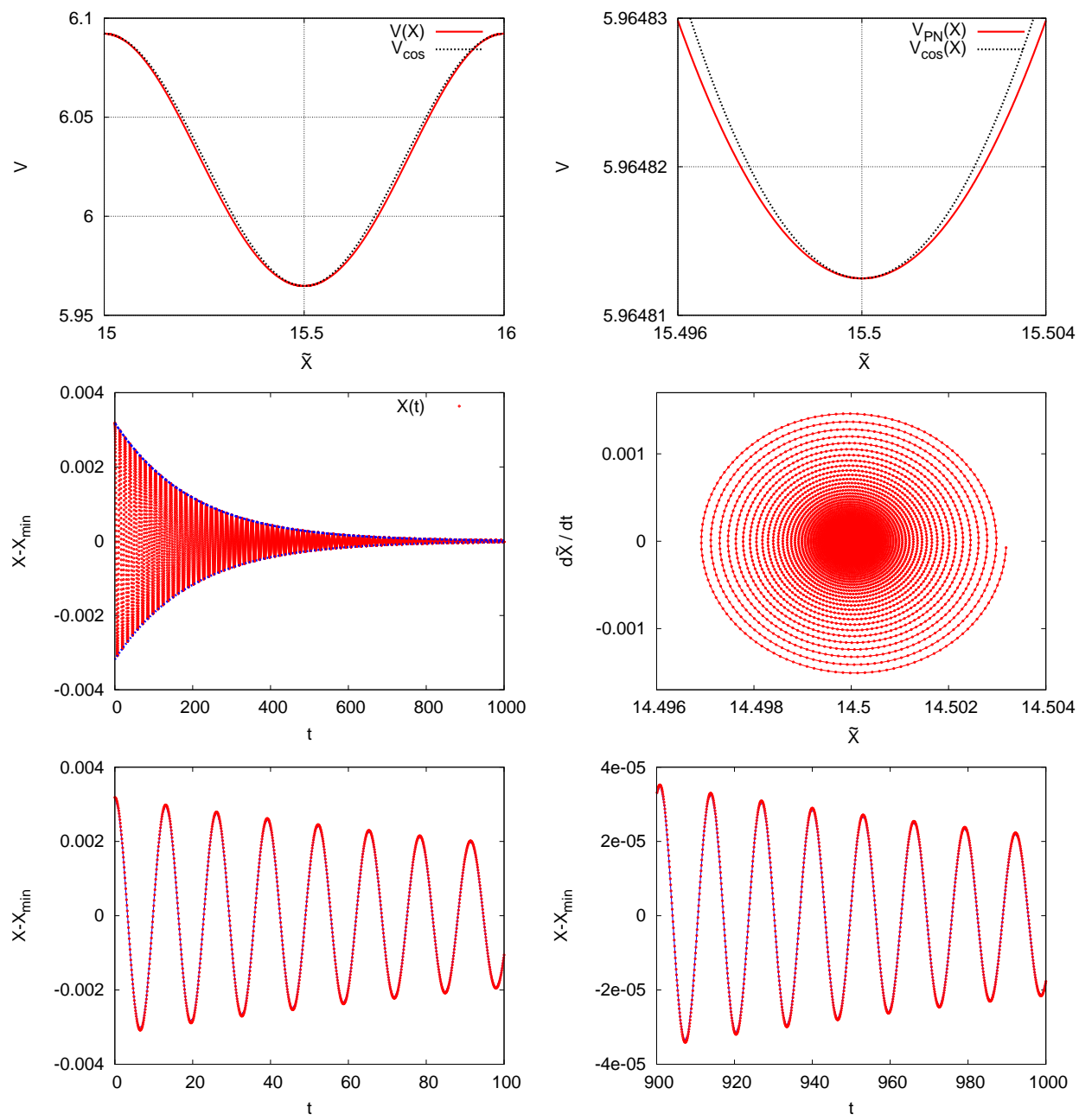

Figura 3.10: Oscilaciones de equilibrio para un fluxón en una red de 60 uniones. Mostramos seis figuras que corresponden al perfil del potencial (arriba), a $X(t)$ (central a la izquierda y abajo) y a $X(V)$ (central a la derecha). 

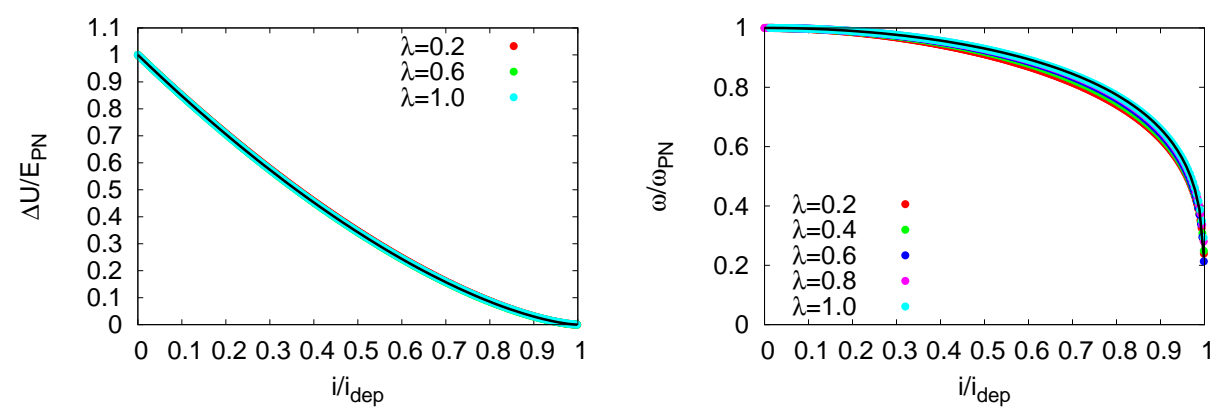

Figura 3.11: Variación de la altura de la barrera y frecuencia característica del mínimo para un fluxón a distintos valores de corriente externa $i$ y para distintos valores del acoplamiento $\lambda$. La línea negra muestra los resultados considerando un potencial sinusoidal inclinado..

pequeñas oscilaciones la dinámica del centro de masa viene dada por

$$
\ddot{X}+\gamma \dot{X}+\omega_{P N}^{2} X=0,
$$

con lo que $X(t)=X_{0} \exp (-\gamma t / 2)\left(\cos \Omega t+\frac{\gamma}{2 \Omega} \sin \Omega t\right) \operatorname{con} \Omega^{2}=\omega_{P N}^{2}-\gamma^{2} / 4$. Las simulaciones se han realizado para $\gamma=0.01$. El resultado de las simulaciones se muestra en la figura 3.10 donde se comprueba un total acuerdo entre lo obtenido en el sistema completo y lo predicho por la imagen de partícula.

\subsubsection{Dependencia con $i$}

Todos los resultados anteriores han sido presentados para el caso $i=0$. La inclusión de un campo externo modifica las propiedades del fluxón. Siguiendo nuestra imagen de partícula el efecto de la corriente es inclinar el potencial de Peierls-Nabarro, $V(X ; i)=V(X, 0)-i X$, reduciendo el valor de la barrera y de la frecuencia que se anulan para la fuerza crítica o $i_{d e p}$. Según esta imagen la altura de la barrera

$$
\Delta U(\lambda, i)=E_{P N}\left(\sqrt{1-f^{2}}-f \cos ^{-1} f\right)
$$

y la frecuencia en el mínimo

$$
\omega_{m i n}(\lambda, i)=\omega_{P N}\left(1-f^{2}\right)^{1 / 4}
$$

con $f=i / i_{\text {dep }}$. La figura 3.11 muestra los resultados numéricos para distintos valores del $\lambda$ y los correspondientes a las fórmulas anteriores encontrándose un acuerdo excelente.

Con respecto a la masa, en principio esta también puede depender de la corriente externa aplicada. No obstante, tomando como referencia la definición 


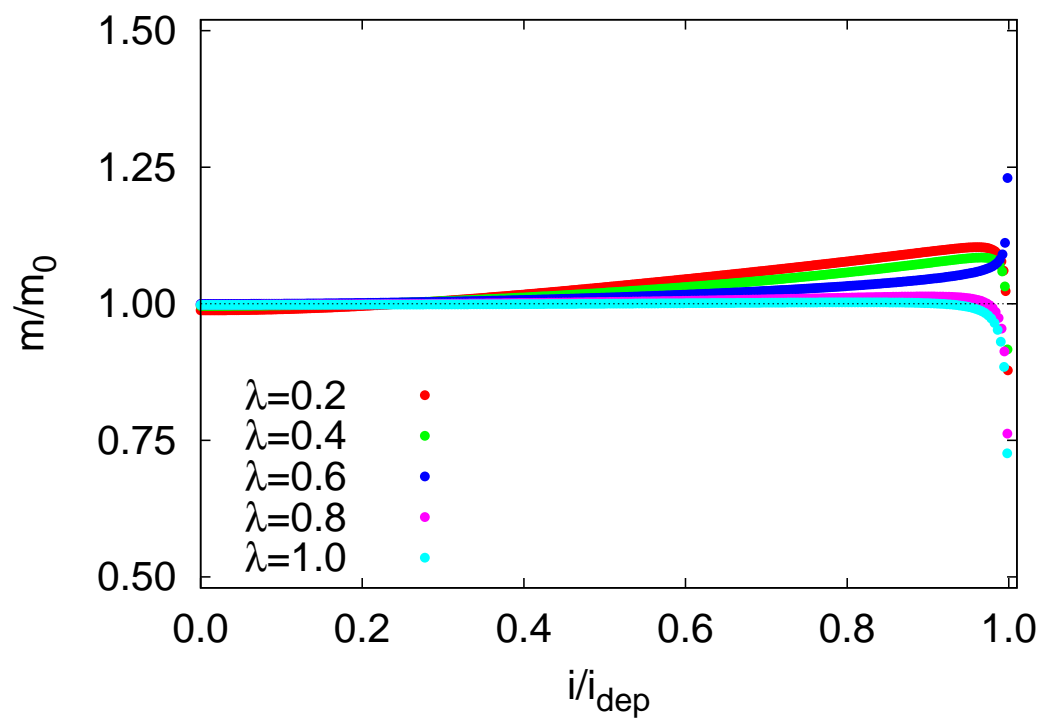

Figura 3.12: Calculo de la dependencia de la masa efectiva del fluxón $m(i)$, a distintos valores de corriente externa $i$ y para distintos valores del acoplamiento $\lambda$.

de $m_{\min }$ es posible ver que la dependencia con la cual la segunda derivada del potencial se anula cuando $i \rightarrow i_{d e p}$ es la misma que la mostrada por $\omega_{P N}$ con lo cual se tendría que $m_{\min }$ no debería cambiar apreciablemente al variar $i$. Podemos apreciar esto en la figura 3.12 en donde hemos calculado la dependencia de la masa con la corriente externa normalizada a la corriente de desanclaje. La expresión de la masa se ha obtenido a partir de la segunda derivada del potencial y el cuadrado de la frecuencia, $m(i)=V_{m i n}^{\prime \prime} / \omega^{2}$ con $V(X) \simeq V_{0}+i_{\mathrm{dep}}(1-\cos X)-i X$.

\subsection{Redes Ratchet}

Las redes ratchet están caracterizadas por poseer potenciales de PeierlsNabarro carentes de simetría de inversión. En capítulos anteriores, hemos visto que la tecnología actual tiene la flexibilidad suficiente para diseñar anillos con uniones de diferentes áreas y celdas de diferentes tamaños. El tamaño de celda es un parámetro importante pues afecta por un lado al valor del coeficiente de autoinducción magnética del sistema y por otro a la magnitud de los flujos debidos a campos magnéticos externos o auntoinducidos. Hemos visto como un análogo físico de nuestro sistema es una cadena de péndulos unidos por muelles de torsión. Entonces el efecto primordial del tamaño de celda 
es variar la constante de los muelles y su longitud natural. Por otro lado el área de la unión afecta al valor de la corriente crítica, capacidad y resistencia de la misma. En nuestra imagen básicamente corresponde a una modificación fundamentalmente de la longitud natural del péndulo su masa y fricción.

El diseño de redes con distintos tamaños de celda y de unión dará lugar a potenciales efectivos no regulares para el fluxón. Verdaderamente, la flexibilidad del sistema es tal que en teoría podemos hablar de que el diseño permite tallar cualquier potencial deseado para los fluxones. De entre todas las configuraciones, llama poderosamente la atención la posibilidad de diseñar redes con un potencial efectivo periódico pero asimétrico para los fluxones: lo que llamaremos un potencial de tipo ratchet. En el capítulo anterior hemos presentado el interés en el estudio de este tipo de sistemas. El anillo de uniones Josephson es un sistema experimental adecuado para estudiar experimentalmente diversos problemas interesantes relacionados con las propiedades dinámicas en sistemas carentes de simetría de inversión. En particular, es especialmente destacable el hecho de que los anillos de uniones Josephson permiten abordar las propiedades dinámicas de objetos espacialmente extendido, un fluxón o solitón en este caso, sobre potenciales efectivos asimétricos.

\subsection{1. $\quad$ Red ratchet de periodo 2}

En su artículo de 1999, F. Falo et al. [83] presentaron una configuración de red con potencial asimétrico basada en la alternancia de celdas de dos tamaños

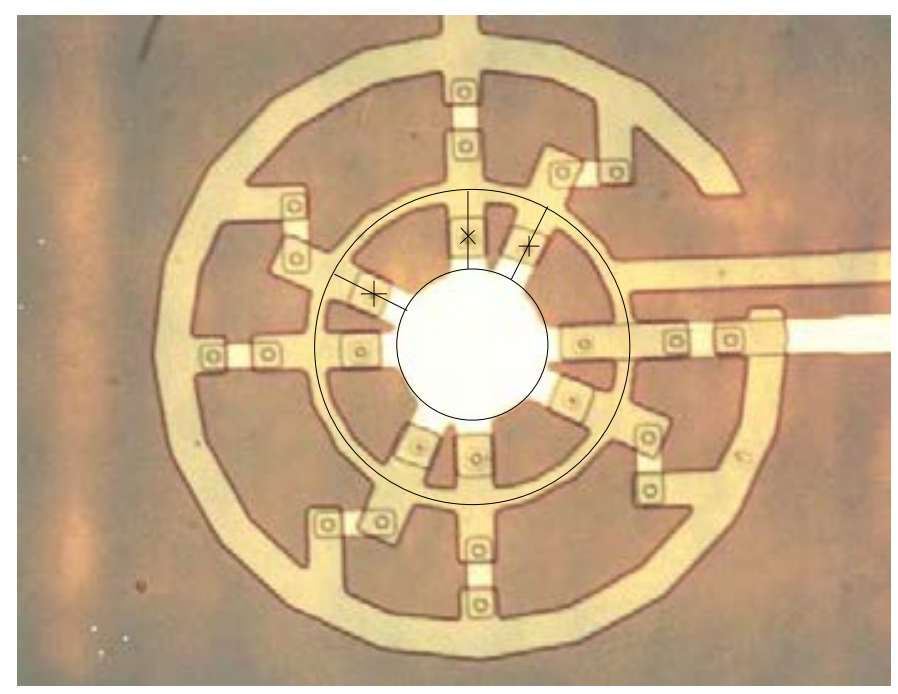

Figura 3.13: Fotografía de un anillo diseñado para estudiar las propiedades de fluxones en potenciales asimétricos. 


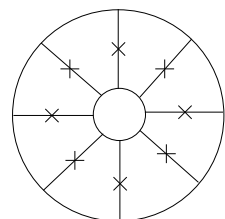

(a)

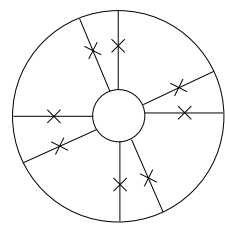

(c)

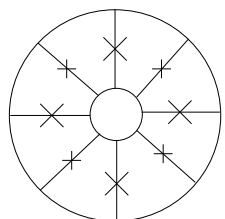

(b)

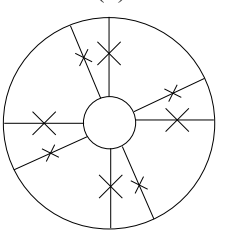

(d)

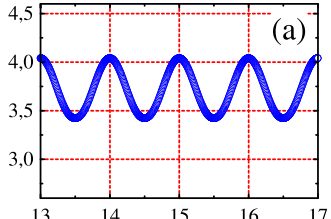

$\sum_{\text {Iิ) }}^{40}$

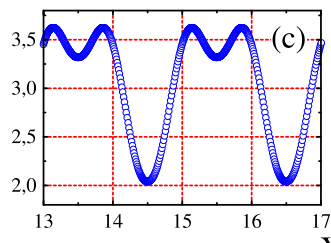

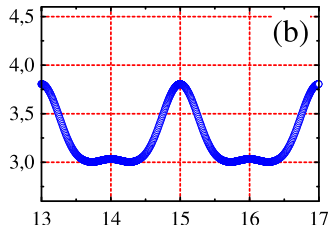

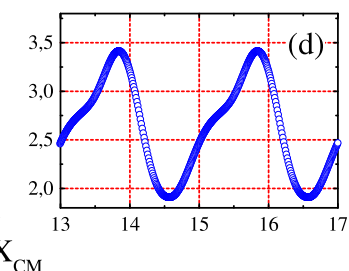

Figura 3.14: Dibujo esquemático de un anillo (a) regular; (b) que alterna uniones de dos tamaños diferentes; (c) que alterna celdas de dos tamaños diferentes; y (d) que alterna celdas y uniones de dos tamaños diferentes. A la derecha se muestra el perfil de energía potencial $E_{P N}(\tilde{X})$ para el fluxón en las redes anteriores. La red (d) corresponde a un perfil de tipo ratchet.

diferentes y uniones de dos áreas distintas. En este caso, en su aproximación más sencilla y despreciando campos externos, la energía del sistema puede escribirse como

$$
\frac{E}{E_{J}}=\sum_{j} h_{j}\left(1-\cos \varphi_{j}\right)+\frac{\lambda_{j}}{2}\left(\varphi_{j+1}-\varphi_{j}\right)^{2}
$$

con $\left\{h_{j}\right\}=\left\{h_{1}, h_{2}, h_{1}, h_{2}, \ldots\right\}$ y $\left\{\lambda_{j}\right\}=\left\{\lambda_{1}, \lambda_{2}, \lambda_{1}, \lambda_{2}, \ldots\right\}$. Dicho sistema fue diseñado, fabricado y medido experimentalmente (ver figura 3.13) verificándose el carácter ratchet para fluxones del mismo [84, 85].

La figura 3.14 muestra esquemáticamente cuatro anillos formados por uniones Josephson y el perfil de energía para un fluxón en cada una de la redes. El caso (a) muestra una red regular y el potencial de Peierls-Nabarro correspondiente es un potencial periódico prácticamente sinusoidal con mínimos asociados a posiciones del fluxón centrado en una celda y máximos para fluxón centrado en la unión $\left(h_{j}=1\right.$ y $\left.\lambda_{j}=0.25, \forall j\right)$. El caso (b) muestra una red donde todas las celdas son iguales pero que contiene uniones de dos tipos $\left(h_{j}=1\right.$ ó 0.5 y $\left.\lambda_{j}=0.25\right)$. En este caso el potencial es periódico con periodo doble del del caso regular y con dos máximos de distinta magnitud, correspondientes a cada una de los tipos de uniones del sistema. La figura (c) muestra una red de uniones idénticas pero con dos espaciados de celda alternantes $\left(h_{j}=1 \mathrm{y}\right.$ $\lambda_{j}=0.5$ ó 0.25 ). El potencial vuelve a tener periodo doble del regular pero sigue siendo simétrico. Por último, las figuras (d) muestran una red formada por uniones de dos áreas distintas y celdas de dos tamaños diferentes $\left(h_{j}=1\right.$ ó 0.5 y $\lambda_{j}=0.5$ ó 0.25$)$. La combinación de ambos factores produce un potencial de 


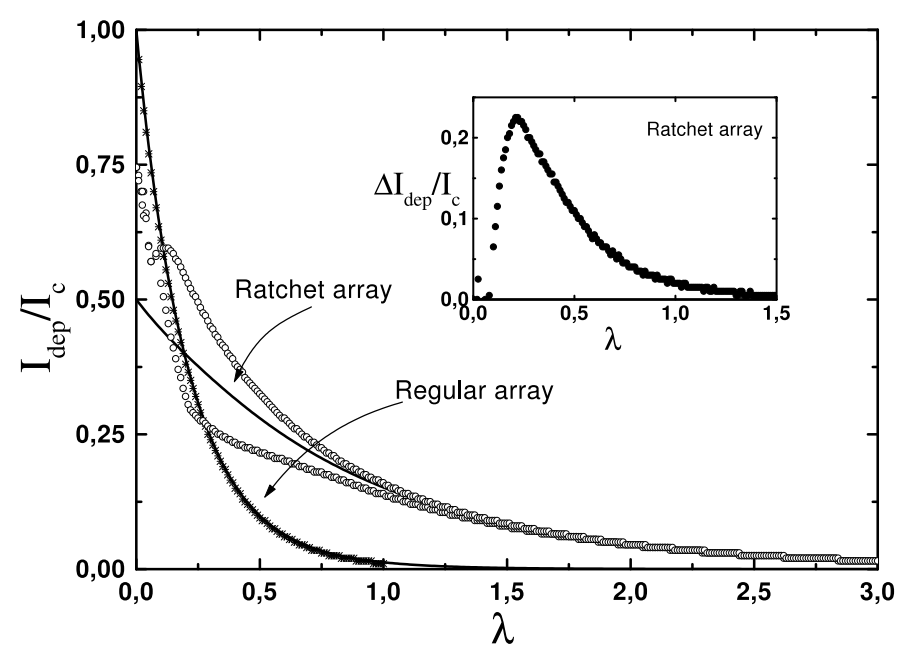

Figura 3.15: Corrientes de depinning $I_{d e p}$ para un fluxón como función de $\lambda$ para la red homogénea y la ratchet $\left\{h_{j}\right\}=\{1,1 / 2,1,1 / 2, \ldots\}$ y $\left\{\lambda_{j}\right\}=\{\lambda, \lambda / 2, \lambda, \lambda / 2\}$. Las líneas continuas muestran las predicciones a partir del valor de $E_{P N}$. Los puntos muestran los resultados numéricos. En pequeño se muestra la diferencia $\left(\Delta I_{d e p}\right)$ entre los valores absolutos de las dos corrientes de depinning para la red ratchet, una manera de cuantificar la magnitud del carácter asimétrico de la red.

periodo doble pero carente de simetría de inversión, potencial de tipo ratchet.

Una de las características más notables desde un punto de vista experimental de las redes asimétricas es la existencia de dos corrientes críticas o corrientes de depinning diferentes para el fluxón, según la corriente alimentada tenga una polaridad u otra. La figura 3.15 muestra el valor de la corriente de las dos corrientes de desanclaje de una red ratchet y una comparación con el resultad de una red regular del mismo $\lambda$ y con el de un potencial sinusoidal de misma barrera pero periodo doble. El inset muestra la diferencia entre las corrientes positivas y negativas, lo que representa una medida efectiva del grado de asimetría del potencial, éste es máximo para valores de $\lambda$ cercanos a 0.2.

\subsection{2. $\quad$ Red ratchet de periodo 3}

La configuración anterior, si bien permite diseñar un potencial asimétrico para el fluxón cuyo periodo es de dos espaciados de red, presenta un problema desde el punto de vista del estudio experimental del sistema. El hecho de que las celdas tengan distinto tamaño modifica los flujos de los posibles campos inducidos en el sistema. Dichas contribuciones fueron eliminadas, por sencillez, de las ecuaciones (3.19) donde se despreciaron esos campos. No obstante, aparecen en una descripción más general como la discutida en la sección 2.1, ver 


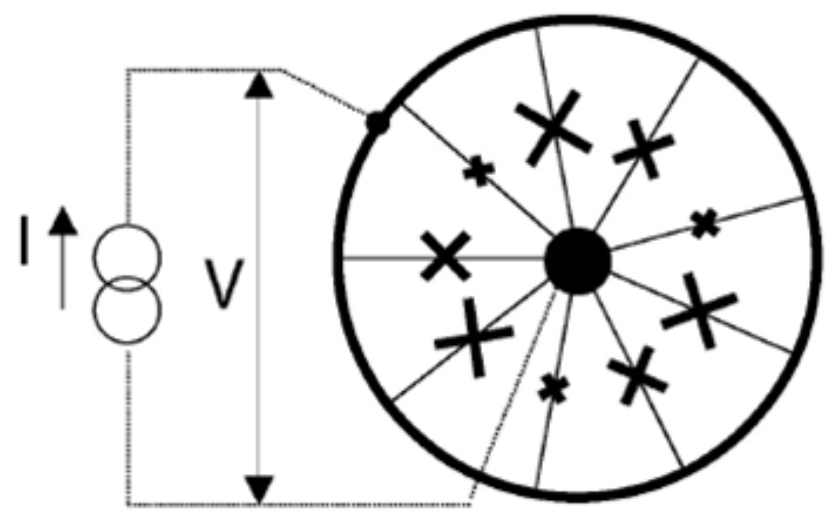

Figura 3.16: Esquema del anillo formado por uniones de tres tamaños diferentes.

ecuación (2.20) por ejemplo.

Por todo ello a continuación, y a lo largo de este trabajo, vamos a plantear una configuración de uniones distintas y celdas idénticas que también posibilita un potencial ratchet para la unión. Dicha configuración consiste en combinar uniones con tres corrientes críticas diferentes, ver figura 3.16. Dicha red tiene una periodicidad de tres celdas y en nuestros estudios usaremos una red con 9 uniones Josephson.

En este caso la energía del sistema puede escribirse como

$$
\frac{E}{E_{J}}=\sum_{j} h_{j}\left(1-\cos \varphi_{j}\right)+\frac{\lambda}{2}\left(\varphi_{j+1}-\varphi_{j}\right)^{2}
$$

con $\left\{h_{j}\right\}=\left\{h_{1}, h_{2}, h_{3}, h_{1}, \ldots\right\}$. Una posible elección, que vamos a usar, es $\left\{h_{j}\right\}=\{1,0.5,0.3, \ldots$.$\} . Dicha configuración posibilita un potencial asimétrico$ para el fluxón a partir de cierto valor del parámetro de acoplamiento $\lambda$.

Para valores pequeños de $\lambda$ el potencial efectivo del fluxón es un potencial periódico de periodo tres pero formado por tres mínimos y máximos diferentes, asociados a cada uno de los tres tipos de uniones. Dicho potencial se va suavizando conforme aumenta $\lambda$ y para un cierto valor crítico $\lambda_{c 1}$ desaparecen uno de esos máximos y mínimos a través de una bifurcación de saddle-node. A partir de entonces se presenta un potencial asimétrico pero formado por dos pozos. Para otro valor acoplamiento mayor, $\lambda_{c 2}$ una nueva bifurcación de saddle-node elimina otro máximo y mínimo y a partir de entonces el potencial efectivo para el fluxón es un potencial asimétrico con un único pozo. Para el caso de la secuencia de $h_{j}$ dada por $(1,0.5,0.3)$ se tiene $\lambda_{c 1} \simeq 0.13$ y $\lambda_{c 2} \simeq 0.23$.

La figura 3.17 muestra el potencial de Peierls-Nabarro para el fluxón y para tres valores diferentes de $\lambda=0.4,0.6$ y 0.8 . En los tres casos se dibuja 


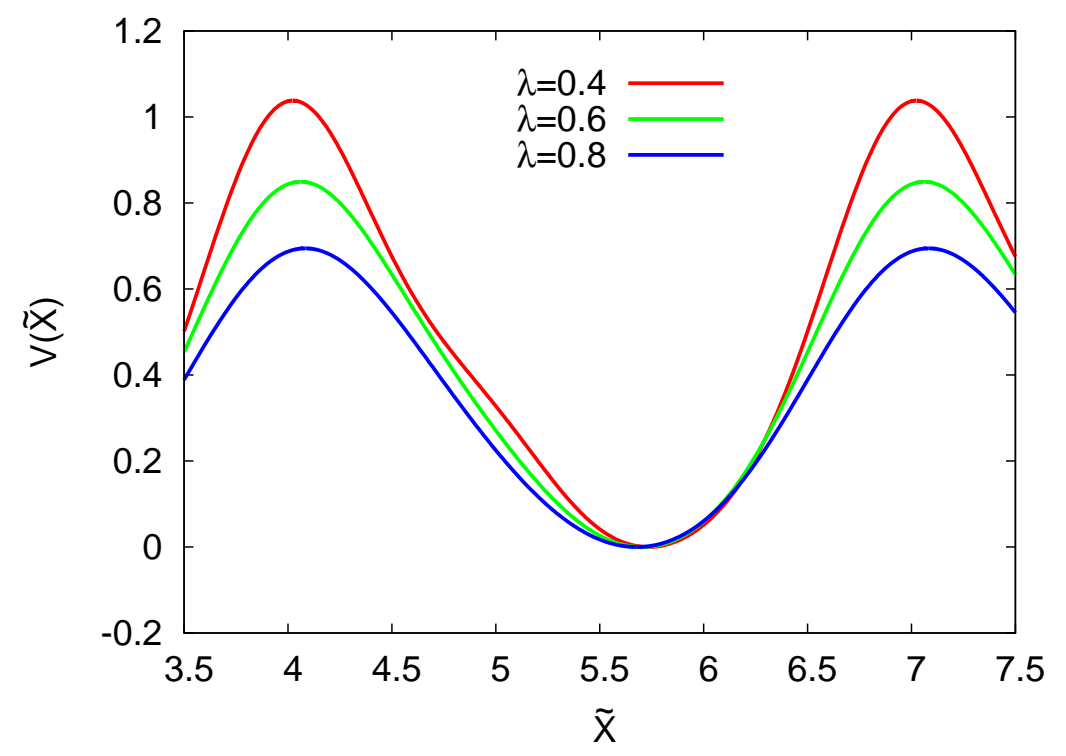

Figura 3.17: Potencial efectivo para el fluxón $V_{P N}(\tilde{X})$ para una red ratchet con $h_{j}=$ $\{1.0,0.5,0.3\}$ y para $\lambda=0.4,0.6,0.8$.

$V(\widetilde{X})-V_{\min }$. La diferencia de energía entre el máximo y el mínimo del potencial define la barrera de Peierls-Nabarro del sistema. La figura 3.18 muestra la dependencia de la barrera de Peierls-Nabarro del sistema en función del parámetro de acoplamiento $\lambda$. Es importante reseñar que en este caso la disminución de $E_{P N}$ con $\lambda$ es mucho más lenta que en el caso de la red regular (comparar con figura 3.2). Por ello, las corrientes críticas de estas redes son mucho mayores que las corrientes críticas de redes regulares con valores de $\lambda$ similares.

Una medida aproximada de la asimetría de la red consiste en calcular la desviación de la diferencia entre la posición del máximo y mínimo del potencial respecto lo esperado para el caso de red regular. Así en el caso de una red regular de periodo tres, dicha diferencia debería ser igual a 1.5. La figura 3.19 muestra el cálculo de la posición del mínimo y el máximo del potencial y la diferencia entre ambos valores. Puede observarse como la asimetría es más acentuada para valores de $\lambda \simeq 0.4$. Este valor nos marca un óptimo en cuanto a la elección de dicho parámetro en el diseño de una red.

Para completar la caracterización de las redes también mostramos la evolución de la frecuencia característica de las oscilaciones de pequeña amplitud del fluxón en torno a su posición de equilibrio, $\omega_{P N}$, figura 3.20. Se puede observar que el comportamiento no es monótono, mostrando un fuerte decrecimiento a valores pequeños de $\lambda$, alcanzándose un agudo mínimo local en torno 


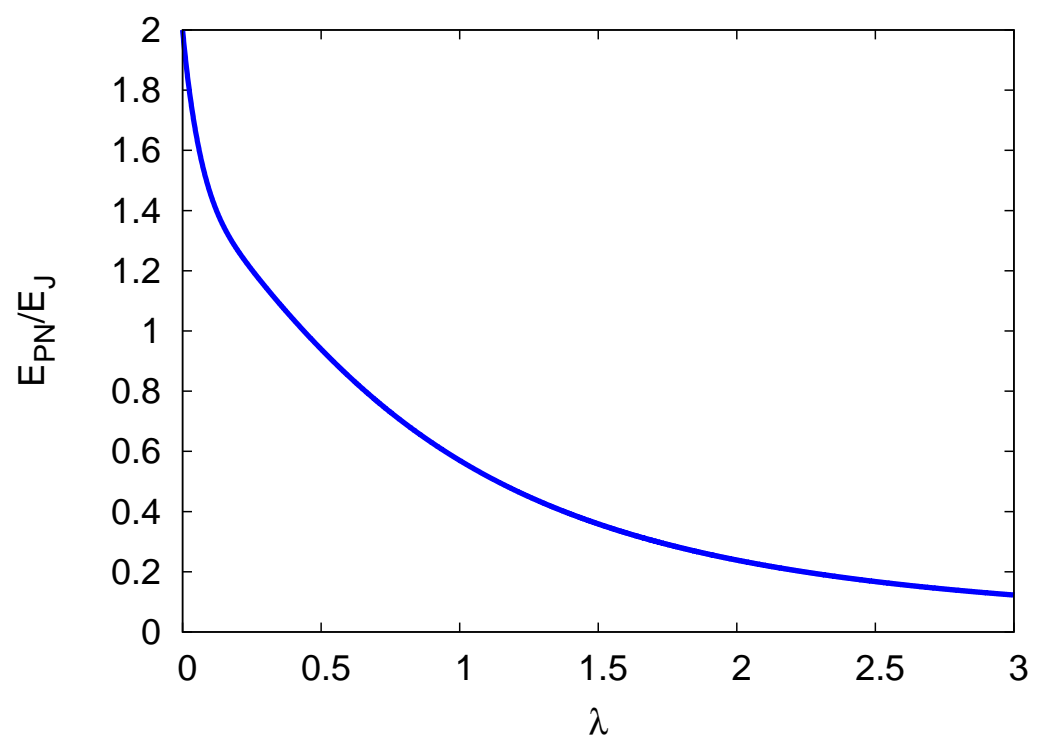

Figura 3.18: Cálculo de la barrera de Peierls-Nabarro, $E_{P N}(\lambda) / E_{J}$ para una red ratchet $\operatorname{con} h_{j}=\{1.0,0.5,0.3\}$.

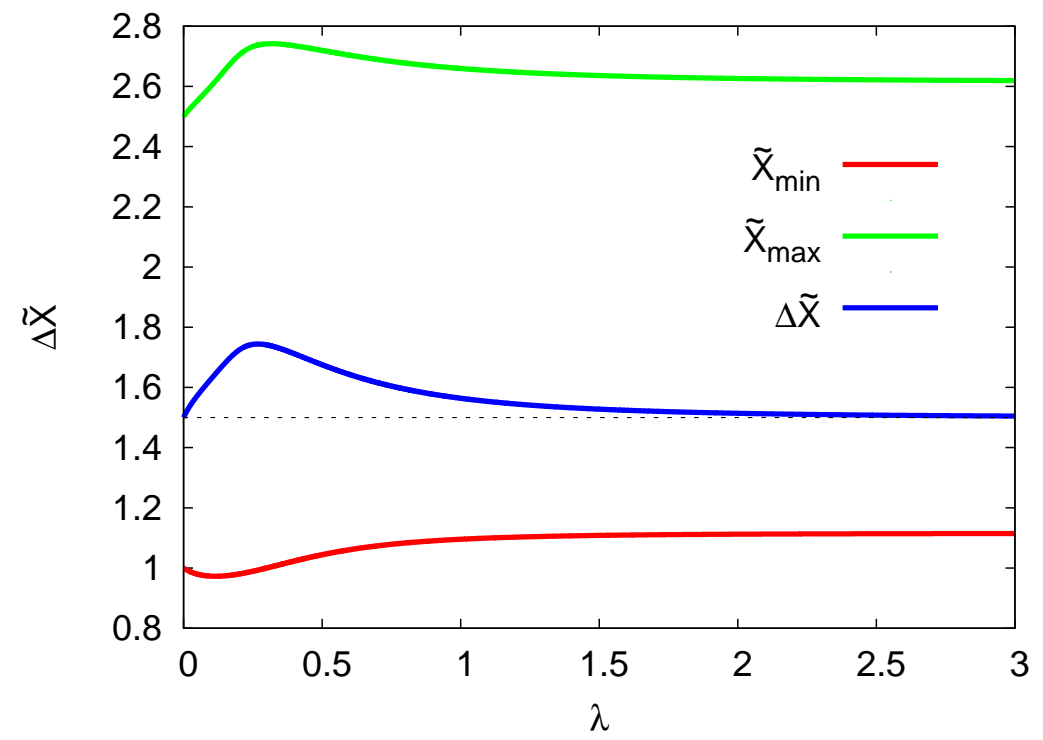

Figura 3.19: Cálculo de $\Delta(\widetilde{X})=\widetilde{X}_{\max }-\widetilde{X}_{\min }$, una medida de la asimetría de la red, como función de $\lambda$ para una red ratchet con $h_{j}=\{1.0,0.5,0.3\}$

a $\lambda \simeq 0.22$, un máximo suave para $\lambda \simeq 0.68$ y decreciendo a partir de entonces. Dicha forma tiene su origen en la paulatina desaparición de mínimos y máximos secundarios al aumentar $\lambda$ para valores pequeños de este parámetro. 


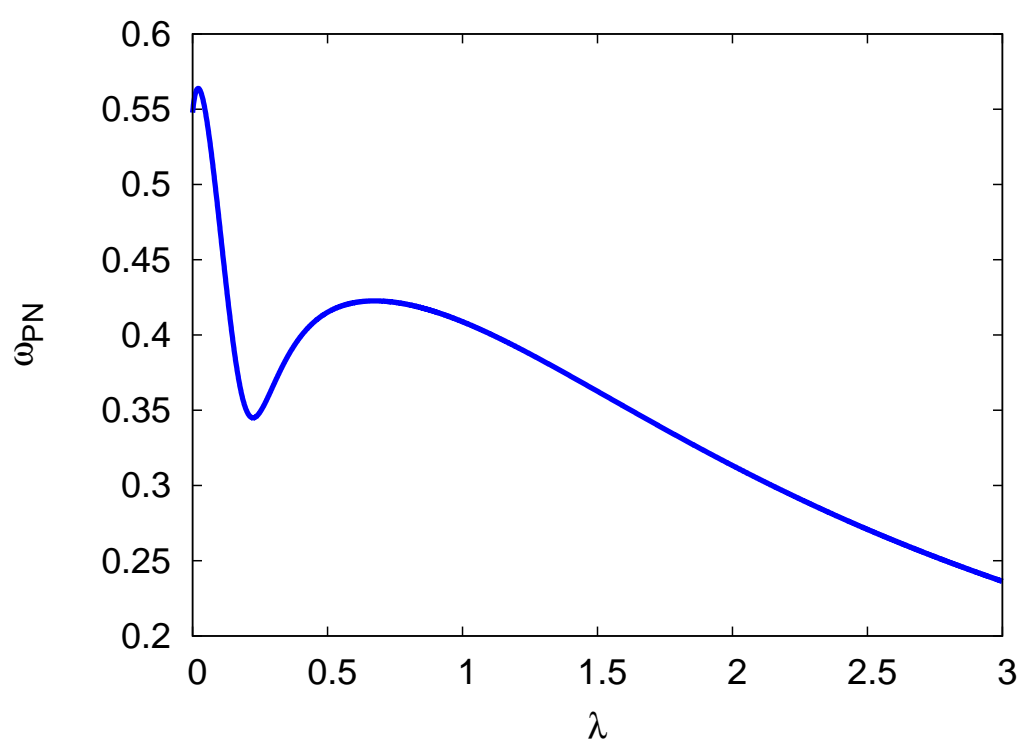

Figura 3.20: Cálculo de la frecuencia de Peierls-Nabarro, $\omega_{P N}(\lambda)$ para una red ratchet con $h_{j}=\{1.0,0.5,0.3\}$.

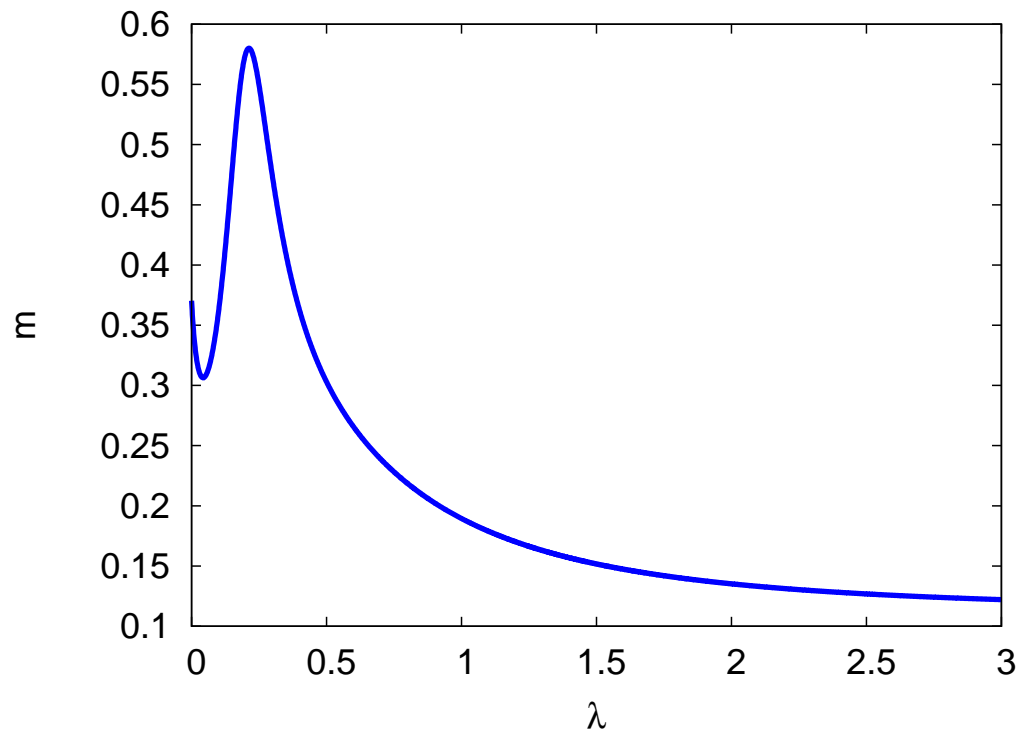

Figura 3.21: Cálculo aproximado de la masa efectiva del fluxón en el mínimo del potencial para una red ratchet $\operatorname{con} h_{j}=\{1.0,0.5,0.3\}$.

Si ahora utilizamos la expresión más sencilla a nuestro alcance para el cálculo de la masa efectiva $m \simeq E_{P N} /\left(2 \times 9 \omega_{P N}^{2}\right)$ (el factor 9 tiene su origen en el periodo triple del potencial efectivo), figura 3.21, observamos el compor- 


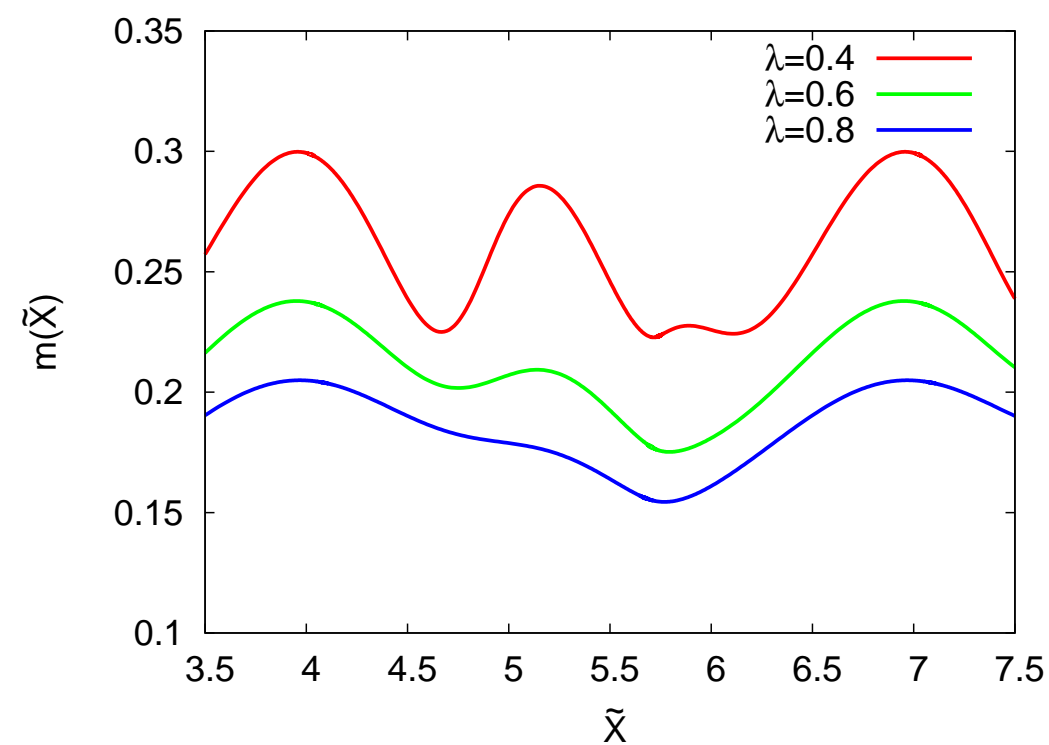

Figura 3.22: Masa efectiva del fluxón $m(\widetilde{X})$ para una red ratchet con $h_{j}=\{1.0,0.5$, $0.3\}$ y para $\lambda=0.4,0.6,0.8$.

tamiento no monótono relacionado con la variación de $\omega_{P N}$.

Resulta muy interesante preguntarse por la evolución de la masa en función de la posición del centro de masas del fluxón, $m(X)$. La figura 3.22 muestra dicho comportamiento para $\lambda=0.4,0.6,0.8$. Es interesante observar la complejidad de dicha dependencia con la existencia de varios mínimos y máximos locales a valores pequeños de $\lambda$ y su desaparición paulatina conforme aumenta el valor de la constante de acoplamiento de la red.

Desde un punto de vista práctico, la principal consecuencia del carácter asimétrico de la red es la existencia de dos corrientes de desanclaje del fluxón, o corrientes de depinning, diferentes según la polaridad de la fuerza externa. La figura 3.23 muestra el resultado de calcular ambos valores, $i_{d e p}^{+}$y $i_{d e p}^{-}$para la red definida por $h_{j}=1 ., 0.5,0.3$ y distintos valores de $\lambda$. La corriente de depinning en un sentido es mayor que en el otro, fruto de que la pendiente del potencial sustrato en ambas direcciones también es distinta.

El hecho de que el potencial sea asimétrico conlleva que ahora cualquier magnitud debe calcularse tanto para corrientes positivas como negativas. El efecto de una corriente externa es "inclinar" el potencial efectivo del fluxón de modo que la barrera de energía del mismo disminuye paulatinamente anulándose al valor correspondiente a la corriente de depinning, ver figura 3.24. Además esta disminución es similar a la que se esperaría para un potencial sinusoidal inclinado (ecuación 3.17) donde se usa el valor adecuado de $i_{d e p}$ para 


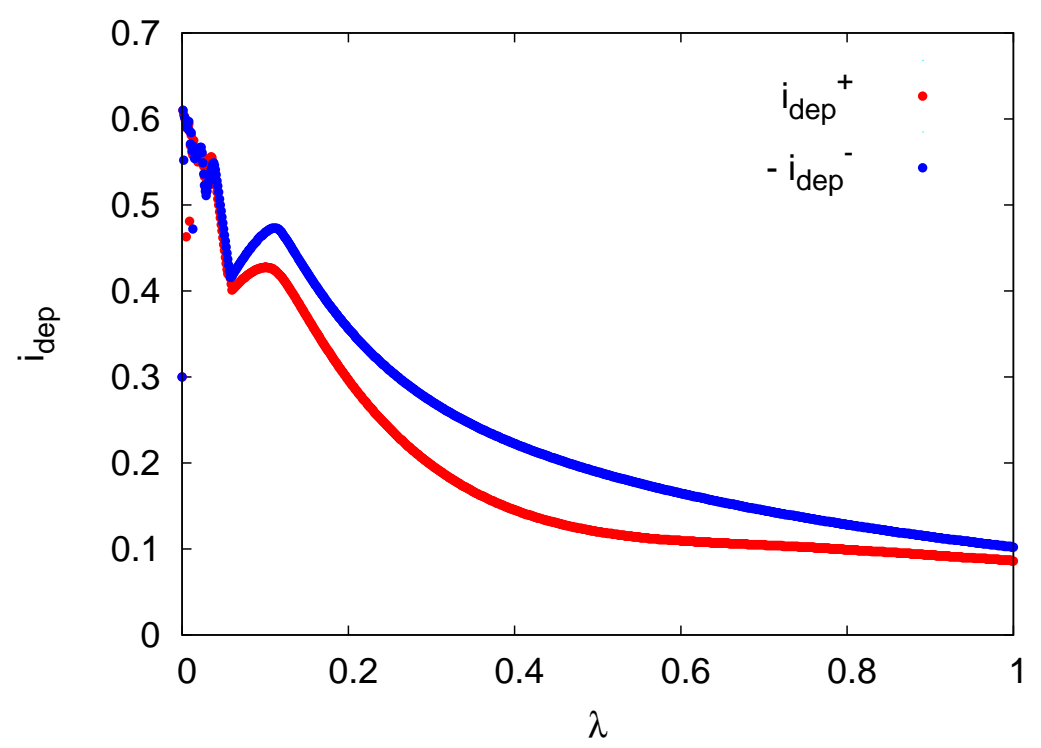

Figura 3.23: Corrientes de depinning $i_{d e p}^{+} \mathrm{y} i_{\text {dep }}^{-}$de un fluxón en una red ratchet: $h_{j}=\{1.0,0.5,0.3\}$ como función del parámetro $\lambda$.

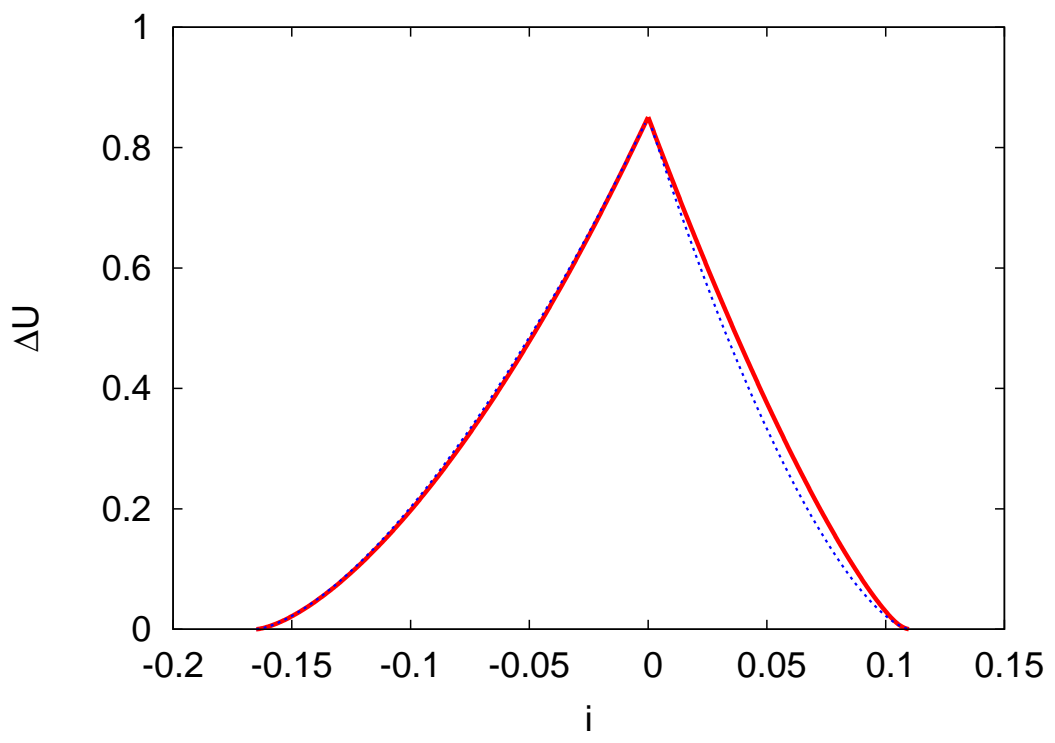

Figura 3.24: Valor de la barrera de Peierls-Nabarro para distintos valores de la corriente externa $i . h_{j}=\{1.0,0.5,0.3\}$ y $\lambda=0.6$.

cada caso. Sin embargo, si estudiamos la dependencia de la frecuencia de oscilaciones del fluxón en torno al mínimo, el comportamiento no es nada trivial, ver figura 3.25. Por un lado al aumentar la corriente se observa un mínimo y 


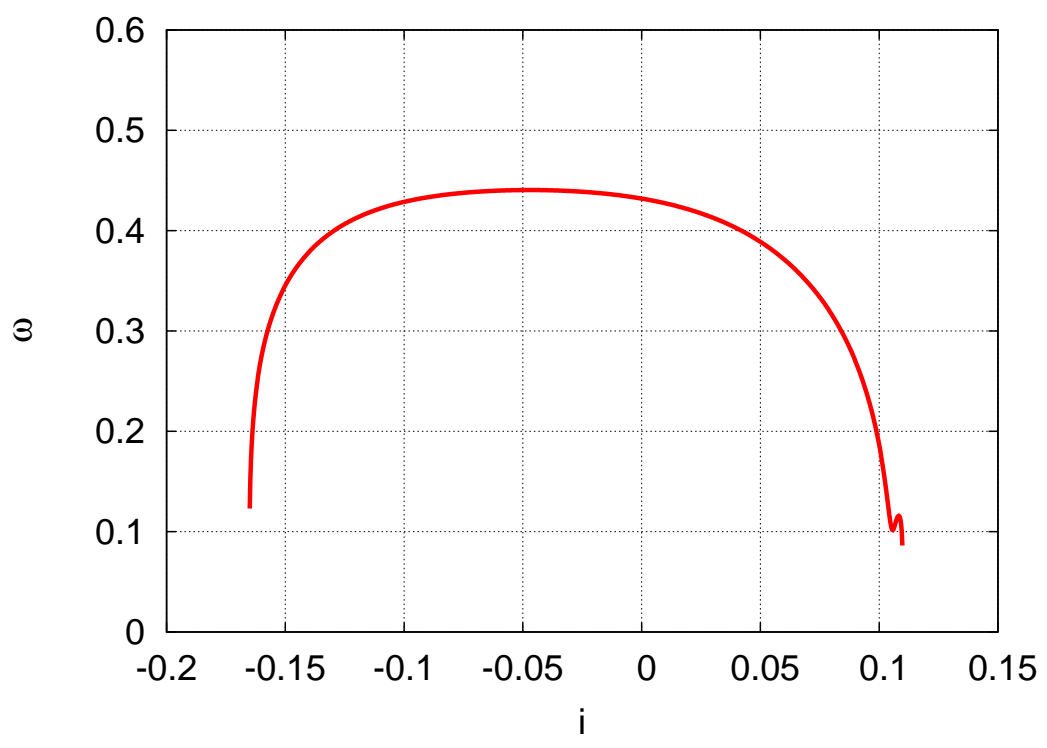

Figura 3.25: Valor de la frecuencia de Peierls-Nabarro para distintos valores de la corriente externa $i . h_{j}=\{1.0,0.5,0.3\}$ y $\lambda=0.6$.

máximo local en la curve de $\omega(i)$ cerca de $i_{d e p}^{+}$. Al disminuir la corriente sorprende el hecho de que $\omega$ primero aumente ligeramente, logrando un máximo para $i \simeq-0.05$ y luego disminuya hacia cero.

En cualquier caso (ver figura 3.26) los potenciales inclinados pueden ser aproximados en las cercanías de los pozos y las barreras de potencial por potenciales cúbicos. 

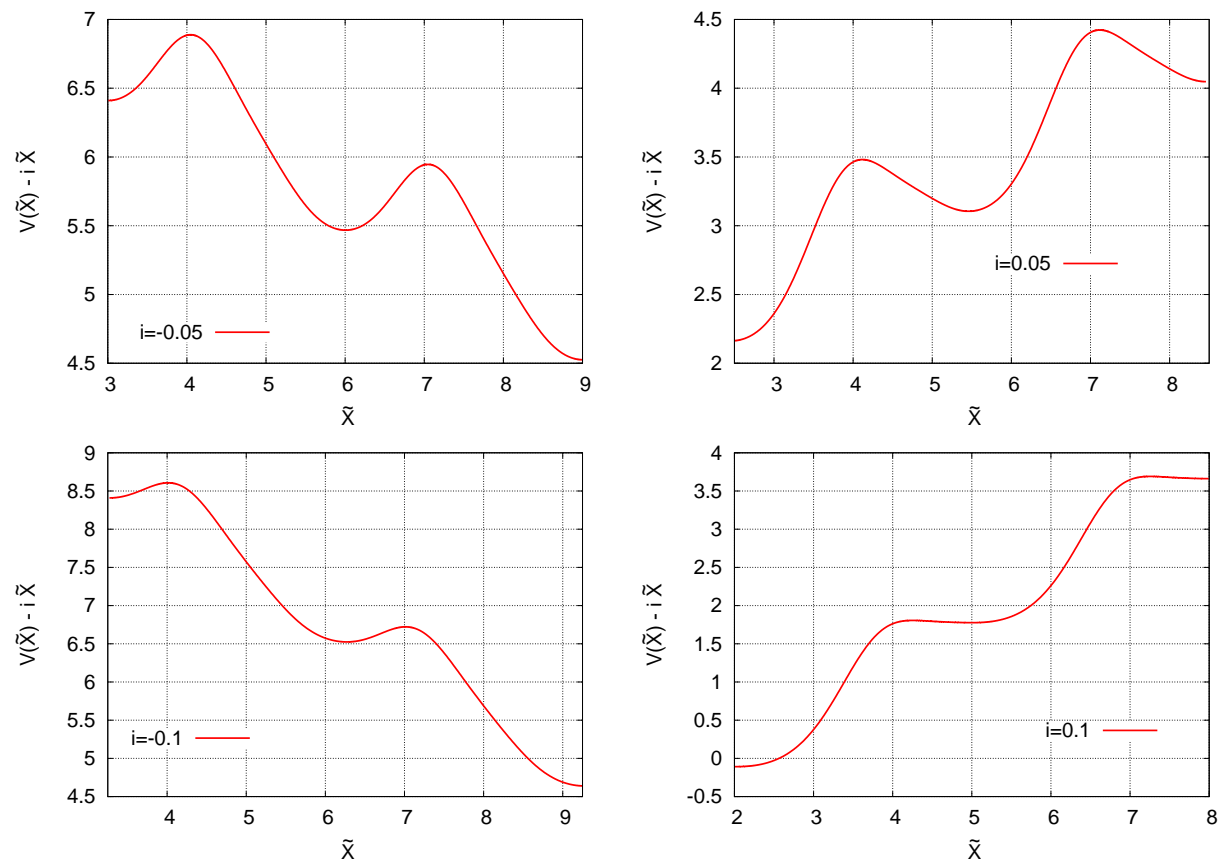

Figura 3.26: Potencial de Peierls-Nabarro $V_{P N}(\widetilde{X})$ del fluxón para cuatro valores de la corriente externa: $i= \pm 0.05$ y $i= \pm 0.1 . h_{j}=\{1.0,0.5,0.3\}$ y $\lambda=0.6$. 


\section{Capítulo 4}

\section{Desanclaje Térmico de Fluxones en Redes regulares}

\subsection{Introducción}

Desde el punto de vista experimental, las uniones Josephson son un sistema privilegiado para el estudio de las propiedades físicas de los solitones y para explorar posibles aplicaciones de las mismas. Como se mencionó en los capítulos anteriores, una red de uniones Josephson con la geometría de anillo se describe muy bien por la ecuación de sine-Gordon discreta y soporta solitones discretos no-lineales o kinks, llamados habitualmente fluxones en este contexto. El estudio y modelización de anillos de uniones Josephson, permite la exploración de nuevos tópicos relacionados con el comportamiento de estos objetos no-lineales.

Al decrecer la temperatura de nuestro sistema por debajo de la temperatura crítica del material superconductor que lo forma, un número en principio indeterminado de fluxones quedan atrapados en el mismo. La presencia de fluxones, domina las propiedades físicas del sistema. A su vez, la dinámica de los mismo también está condicionada por las fluctuaciones térmicas, el intercambio de energía entre el sistema y el ambiente. En particular, dichas fluctuaciones pueden afectar de manera muy importante el valor de la corriente de desanclaje y la forma de curva $I$ - $V$ del sistema.

En este capítulo vamos a considerar la dinámica del desanclaje térmico de fluxones en anillos regulares de uniones Josephson. Nuestro trabajo está motivado por experimentos recientes sobre el desanclaje térmico y la dinámica de fluxones en anillos pequeños conformados por nueve uniones Josephson [90]. Como veremos, debido a las fluctuaciones térmicas un fluxón puede ser ex- 
citado desde su estado de mínima energía y moverse a través del arreglo, lo que genera un voltaje a través de cada unión. En particular, para valores del amortiguamiento suficientemente pequeños, este desanclaje va asociado a un salto, switching, a la rama resistiva óhmica de la curva $I-V$ del sistema. Si el amortiguamiento es grande la curva $I$ - $V$ es continua y es mucho más difícil determinar el valor de la corriente de desanclaje, en cuanto el voltaje medido nunca es exactamente nulo y la definición del desanclaje puede depender del umbral de voltaje que marquemos en nuestro sistema experimental. Por todo ello, nos limitaremos al estudio del desanclaje en sistemas con amortiguamiento pequeño y a la variación de esta corriente de desanclaje o corriente de depinning $i_{d e p}$ con la temperatura.

Como se mencionó en el capítulo 3, en numerosas ocasiones los fluxones se pueden comparar con partículas afectadas por un potencial sustrato periódico. Uno de los objetivos de este capítulo será probar el alcance de esta imagen en cuanto a las propiedades del anillo afectado por fluctuaciones térmicas. En particular compararemos los resultados de la corriente de desanclaje del fluxón en presencia de temperatura, con los resultados para una partícula y a su vez confrontaremos de nuevo todos los resultados obtenidos frente a la predicciones analíticas para el escape de partículas de un pozo metaestable dadas por la teoría de Kramers y sus extensiones. Veremos que para el efecto de desanclaje, los fluxones se comportan como partículas y obedecen leyes de escape tipo Kramers y en este sentido podemos referirnos al desanclaje del fluxón como el escape del fluxón. Sin embargo, bajo algunas condiciones particulares, los resultados encontrados se apartan de este comportamiento de partícula. En particular, para algunos valores de los parámetros el fluxón puede sufrir difusión a bajo voltaje antes de cambiar al modo óhmico ó whirling de alto voltaje. En este caso el proceso de depinning corresponde a un salto a la rama de difusión del sistema y para valores mayores de la corriente se observa un segundo salto, que denominamos aquí como proceso de switching y que corresponde al paso de la rama de difusión a la rama óhmica del sistema.

El hallazgo de esta rama de difusión es un resultado singular. Por un lado fenómenos de difusión no son esperados en estos sistemas a valores pequeños del amortiguamiento y los resultados muestra que es necesario una temperatura suficientemente alta para que aparezcan. Por otro lado este fenómeno no se observa para el caso de una partícula subamortiguada a ningún valor de la temperatura.

Este capítulo está dedicado al estudio de la dinámica de anillos regulares de uniones Josephson. Como hemos visto, una unión Josephson es una versión experimental de un péndulo no lineal y un anillo lo es de una cadena cerrada de péndulos acoplados por muelles de torsión. Las ecuaciones que gobiernan la 
dinámica de la red, en su aproximación más sencilla son:

$$
\ddot{\varphi}_{j}+\Gamma \dot{\varphi}_{j}+\sin \varphi_{j}+\tilde{i}_{j}(\tau)=\lambda\left(\varphi_{j+1}-2 \varphi_{j}+\varphi_{j-1}\right)+i
$$

donde $j=1, \ldots N$ y $\varphi_{j}$, la variable que describe el comportamiento de la jésima unión, es la diferencia de fase invariante gauge del parámetro de orden superconductor a ambos lados de la unión. En esta ecuación la corriente esta normalizada por la corriente crítica de cada unión $I_{c}$ y el tiempo por la frecuencia de plasma $\omega_{p}=\sqrt{2 \pi I_{c} / \Phi_{0} C}\left(\Phi_{0}=h / 2 e\right.$ es el cuanto de flujo magnético y $C$ es la capacitancia de la unión). $\Gamma$ es el parámetro que mide la disipación en el sistema $\left(\Gamma=\sqrt{\Phi_{0} / 2 \pi I_{C} C R^{2}}\right.$, con $R$ la resistencia efectiva de la unión). El término, $\tilde{i}(\tau)$, describe el efecto del ruido térmico en la dinámica (ruido tipo Johnson) y satisface $\left\langle\tilde{i}_{j}(\tau)\right\rangle=0$ y $\left\langle\tilde{i}_{j}(\tau) \tilde{i}_{k}\left(\tau^{\prime}\right)\right\rangle=2 \Gamma T \delta_{j k} \delta\left(\tau-\tau^{\prime}\right)$ donde usamos $T$ para la temperatura normalizada $T=k_{B} T_{\exp } / E_{J}$ (con $E_{J}$ la energía Josephson $\left.E_{J}=\Phi_{0} I_{C} / 2 \pi\right)$. El voltaje normalizado $v$ muestra la respuesta del sistema a la corriente externa, está definido por $v=V_{d c} / I_{c} R=$ $\left(\Phi_{0} / 2 \pi I_{c} R\right)\langle d \varphi / d t\rangle=\Gamma\langle d \varphi / d \tau\rangle$. En las gráficas en las que se muestra la curva $I$ - $V$ del anillo dibujaremos en realidad la curva $i$ - $v$ del mismo; es decir, dibujamos corriente y voltajes normalizados.

El parámetro $\lambda$ representa el acoplamiento entre las uniones, las cuales, en el marco de este modelo, se presenta entre los vecinos y tiene un carácter inductivo $\lambda=\Phi_{0} / 2 \pi I_{c} L$, con $L$ la auto inductancia de cada celda en el arreglo. Remarcamos que las condiciones de frontera están definidas por la topología del arreglo. Aquí hemos considerado arreglos circulares de modo que $\varphi_{j+N}=$ $\varphi_{j}+2 \pi M$ donde el número $M$ de fluxones atrapados en el sistema es una constante del movimiento.

Vamos a considerar el estudio de redes relativamente pequeñas, 9 uniones, con uno, dos y tres fluxones, aunque centraremos la parte fundamental del análisis en el sistema con un fluxón. En este caso también presentaremos resultados para tamaños mayores del arreglo. No obstante nuestro trabajó se dirigió principalmente a arreglos hechos de 9 uniones, similares a aquellos que están siendo experimentalmente estudiados. Después de simular un amplio rango de valores en los parámetros del sistema, como el acoplamiento $\lambda$, el amortiguamiento $\Gamma$ y temperatura, por ejemplo, seleccionamos y restringimos nuestro interés a arreglos discretos $(\lambda<1)$ y subamortiguados, barriendo un amplio rango de temperaturas.

A modo de ejemplo la figura 4.1 muestra las configuraciones de equilibrio para uno, dos y tres fluxones en un anillo de nueve uniones Josephson con $i=0$ y para dos valores del acoplamiento, $\lambda=0.2$ y $\lambda=0.8$. A $\lambda$ bajo el fluxón se asemeja a una función escalón, su anchura es pequeña, pero a $\lambda$ alto la anchura del mismo es mayor y las fases cambian de un modo más suave a lo largo del anillo. 

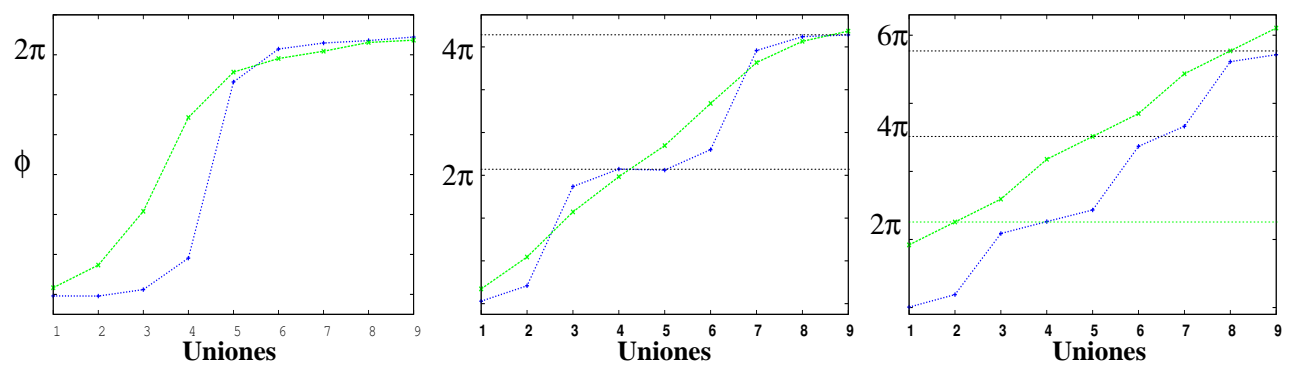

Figura 4.1: Perfil de mínima energía para uno (izquierda), dos (centro) y tres (derecha) fluxones en un anillo de nueve uniones Josephson con $\lambda=0.2$ (azul) y $\lambda=0.8$ (verde).
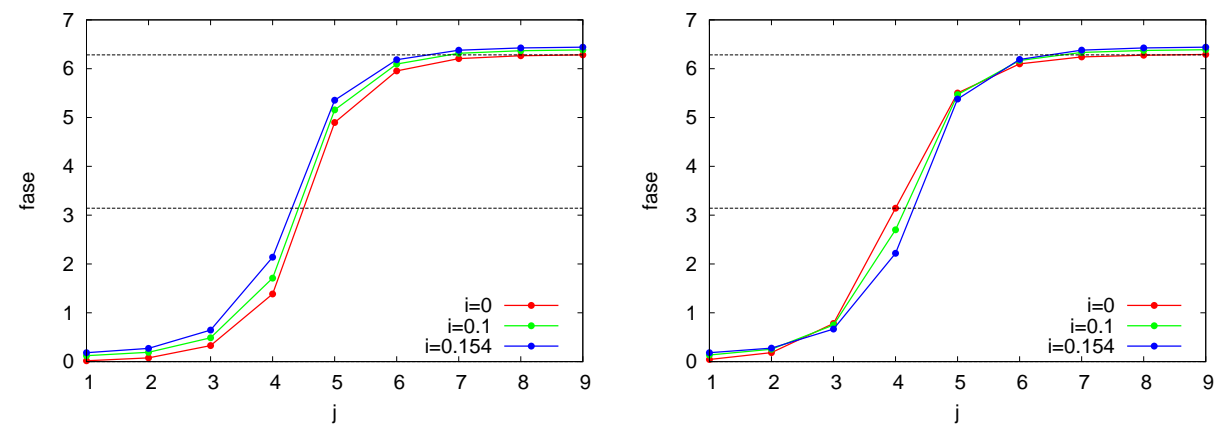

Figura 4.2: Perfil del fluxón en el mínimo (izquierda) y máximo (derecha) del potencial de Peierls-Nabarro para una red regular con 9 uniones y $\lambda=0,4$ y a tres valores distintos de la corriente externa $i=0,0.1,0.154$.

Como en el caso experimental, en nuestro arreglo de uniones comenzamos con estado de voltaje cero $V=0$ y corriente cero $I=0$ e incrementamos suavemente la corriente a un determinado ritmo. La velocidad de cambio de la corriente o rampa es un parámetro adicional que como veremos tiene su importancia. Al aumentar la corriente el fluxón se va deformando, la barrera de Peierls-Nabarro del mismo va disminuyendo y para un determinado valor de $i$, la corriente de depinning o desanclaje a temperatura cero, la barrera se anula y el fluxón pasa de un estado de $V=0$ a un estado de $V \neq 0$. Este cuadro se ve modificado por la temperatura, que provoca el escape prematuro del fluxón, a barreras no nulas, asistido por las fluctuaciones térmicas. Como hemos indicado la forma del fluxón también varía en la medida que la fuerza externa aumenta sin que este comience a moverse como un todo. Como ilustración la figura 4.2 muestra el perfil de mínima energía (izquierda) y el de máximo local (derecha) de un fluxón en una red con $\lambda=0.4$ para distintos valores de la corriente externa. Podemos apreciar que este cambia muy ligeramente al aumentar $i$ y máximo y mínimo coinciden a la corriente de depinning, en este caso $i_{d e p} \simeq 0.1544$. Por encima de la corriente de desanclaje y a bajo amorti- 
guamiento, la curva $I$ - $V$ entra en la rama resistiva y el fluxón se deslocaliza a lo largo de la red.

\subsection{Red regular con 9 uniones y un fluxón}

En esta sección mostramos las simulaciones numéricas de la dinámica de un fluxón en un anillo en arreglo regular de 9 uniones Josephson y una partícula en un potencial periódico. Comparamos los resultados con los cálculos numéricos de la teoría de escape térmico de una partícula en un potencial metaestable. Los resultados se han obtenido integrando numéricamente las ecuaciones de la dinámica del sistema utilizando un método de Runge-Kutta determinista o estocástico según el caso.

\subsubsection{Consideraciones sobre la rampa}

Antes de discutir las curvas $I$ - $V$ obtenidas merece la pena considerar brevemente la influencia de la elección de la rampa en las simulaciones. En nuestra simulaciones fijamos un valor de la corriente externa, integramos las ecuaciones dinámicas durante un tiempo transitorio y luego promediamos sobre un tiempo para calcular el voltaje medio del sistema a ese valor de la corriente externa. Posteriormente se incremente la corriente por una cantidad pequeña $\delta i$ y se vuelve a calcular el voltaje. La condición inicial es un fluxón en configuración de mínima energía a $i=0$ y para cada nuevo valor de $i$ se toma la configuración final de fases y velocidades de la corriente anterior. De este modo se simula un cambio adiabático de la corriente externa y una medida del voltaje dc del sistema para cada corriente. Pongamos por caso una simulación donde se integran 200 puntos de corriente entre 0 y 0.2 con lo que $\delta i=10^{-3}$ y en cada corriente se corre el algoritmo $10^{5}$ pasos $\left(3 \times 10^{4}\right.$ de transitorio y $7 \times 10^{4}$ de estacionario por ejemplo) con un paso de tiempo $\delta t=0.01$ con lo que el tiempo de integración total por punto de corriente es de 1000 unidades. Entonces la rampa utilizada es rampa $=10^{-3} / 1000=10^{-6}$.

La rampa es un parámetro más de nuestra simulaciones, del mismo modo que es un parámetro más de los resultados experimentales. No obstante, a temperatura cero, los resultados deben ser independientes de la rampa siempre que esta sea lo suficientemente lenta como para garantizar que los promediados se realizan sobre un verdadero estado estacionario y durante un tiempo suficientemente largo. Esto no es así en el caso de simulaciones con temperatura. En este caso las fluctuaciones siempre están jugando un papel dinámico y sus resultados se observan con mayor o menor probabilidad según el tiempo de observación empleado; es decir, según la rampa utilizada. Por ello estamos in- 

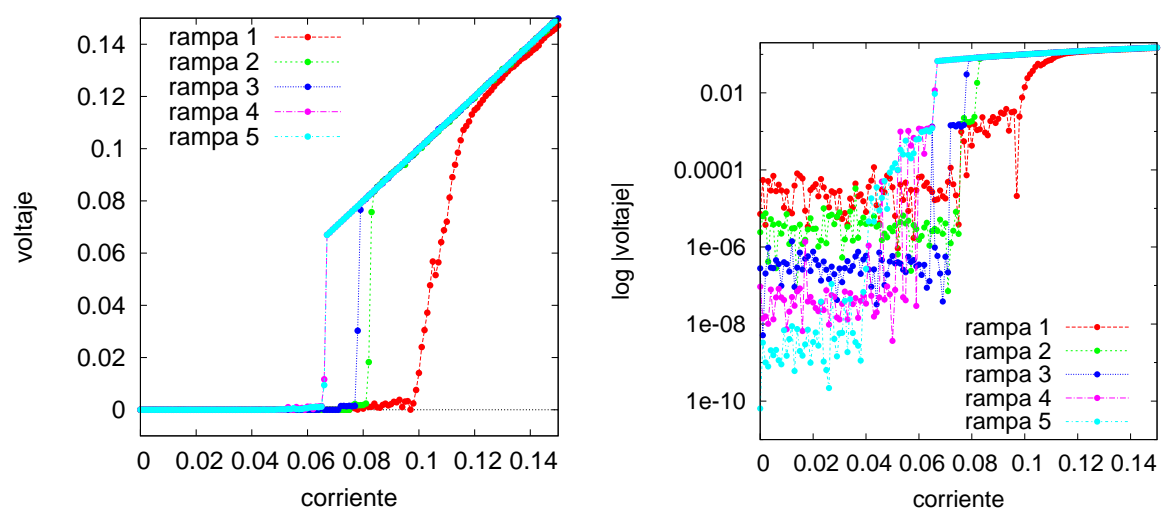

Figura 4.3: Curva $i-v$ del fluxón en un arreglo de 9 uniones para 5 diferentes valores de la rampa, temperatura $T=0.02$, acoplamiento $\lambda=0.4$ y amortiguamiento $\Gamma=0.01$.

teresados en principio en utilizar rampas lentas, pero por otro lado una rampa lenta implica un tiempo de simulación mucho mayor, en algunos casos impracticable. De este modo nos encontramos ante el compromiso de usar rampas pequeñas pero suficientemente grandes para poder realizar la simulaciones requeridas. Esto ha llevado que algunos de los puntos mostrados a lo largo de esta tesis hayan supuesto días de simulación.

A modo de ejemplo mostramos en la figura 4.3 los resultados de la curva $I$ - $V$ de un fluxón en una red con $\lambda=0.4$ y 9 uniones. $\Gamma=0.01, T=0.02$ y se muestran resultados para los siguientes 5 valores de la rampa (cambiando en 5 ordenes de magnitud): A la izquierda se muestra la curva en escala normal y

\begin{tabular}{ccccc}
\hline rampa 1 & rampa 2 & rampa 3 & rampa 4 & rampa 5 \\
$1 / 3 \times 10^{-4}$ & $1 / 3 \times 10^{-5}$ & $1 / 3 \times 10^{-6}$ & $1 / 3 \times 10^{-7}$ & $1 / 3 \times 10^{-8}$ \\
\hline
\end{tabular}

a la derecha en escala logarítmica para el valor absoluto del voltaje. Podemos ver como la curva está caracterizada por una rama de voltaje casi nulo, otra de voltaje pequeño y la rama resistiva. Las transiciones de una rama a la siguiente están fuertemente afectadas por la rampa. También es interesante apreciar que la rama de $v=0$ en realidad es una rama de muy bajo voltaje, obtenido por errores de promediado originados por las fluctuaciones térmicas. Dicho de otra manera esa rama nos da la precisión de nuestro cero de voltaje (se obtienen tanto valores positivos como negativos para $v$ aunque en la figura se ha mostrado el valor absoluto de $v$ para mayor claridad). La figura refleja claramente la importancia de seleccionar la rampa adecuada. Se puede apreciar que para los valores menores de la rampa (rampa 1 y rampa 2) las fluctuaciones por efecto del ruido sobre el movimiento del fluxón son grandes y dificultan 

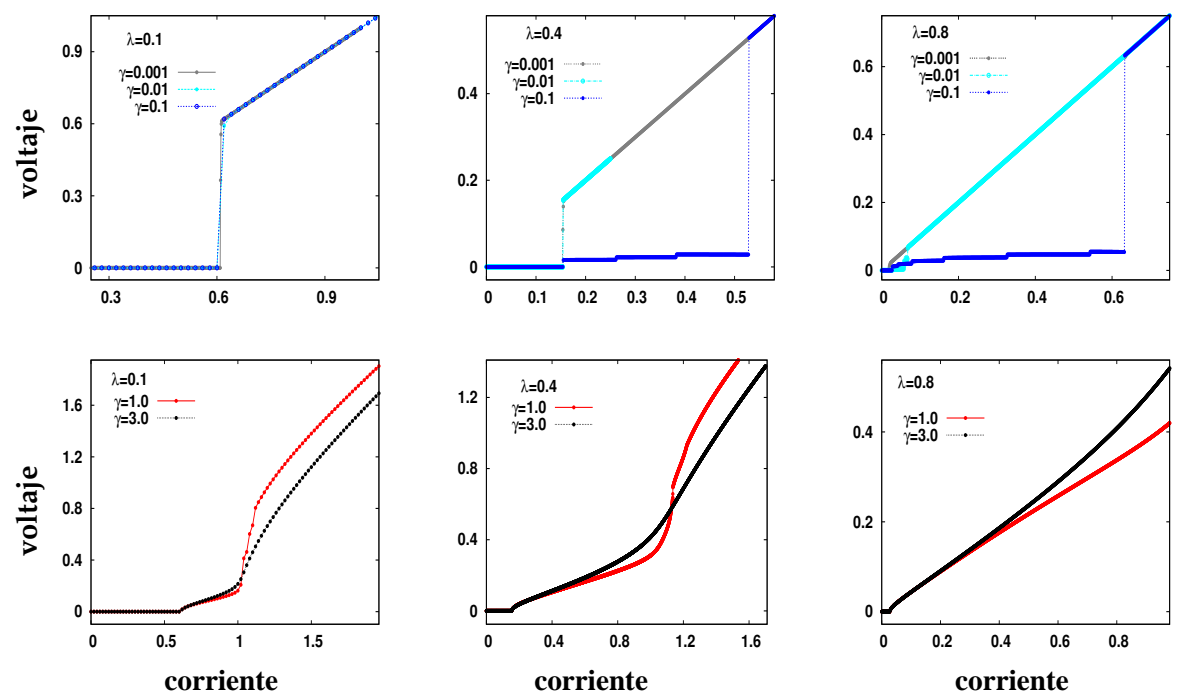

Figura 4.4: Curvas $i-v$ de un fluxón en un arreglo de 9 uniones para diferentes valores del amortiguamiento a temperatura cero y tres valores del acoplamiento; a la izquierda $\lambda=0.1$, en el centro $\lambda=0.4$ y a la derecha $\lambda=0.8$.

definir la corriente a la cual el fluxón comienza a moverse. En la curva que representa el logaritmo del voltaje contra la corriente se ve que con valores altos de la rampa no existe un valor definido de la corriente correspondiente con el salto al régimen lineal. También puede verse como las rampas menores (rampa 5 y rampa 6) muestran una rama de difusión del fluxón de muy bajo voltaje (entre $10^{-5}$ y $10^{-3}$ que se confunde fácilmente con la rama de voltaje cero de las rampas mayores. El problema de utilizar rampas tan pequeñas como rampa 5 es que suponen un tiempo de cálculo muy elevado. La rampa intermedia rampa 3 es una buena elección en esa búsqueda de un compromiso entre exactitud y tiempo de cálculo razonable.

\subsubsection{Curvas $I-V$ a temperatura nula: Regímenes de Amorti- guamiento}

Comenzamos mostrando en la figura 4.4 algunos ejemplos de diferentes curvas $I$ - $V$ obtenidas a temperatura cero y para varios valores del amortiguamiento y el acoplamiento del sistema. La simulación se realizó con un valor promedio de la rampa igual a $\frac{2}{3} \times 10^{-6}$. Todas las curvas muestran la rama superconductora de $v=0$ hasta que se alcanza la corriente de depinning del fluxón, que en nuestro caso resulta ser $i_{\text {dep }}^{0} \approx 0.6087$ para $\lambda=0.1, i_{\text {dep }}^{0} \approx 0.155$ para $\lambda=0.4$ y $i_{\text {dep }}^{0} \approx 0.027$ para $\lambda=0.8$. Las curvas superiores muestran los valores más pequeños del amortiguamiento $\Gamma=0.001,0.01$ y 0.1 ; mientras que 
lqas curvas inferiores muestran el resultado para $\Gamma==1.0$ y 3.0. Para valores pequeños del amortiguamiento (curvas superiores) se observa que las transiciones entre las distintas ramas de la curva $i-v$ son abruptas, están marcadas por discontinuidades en el voltaje. Dichas discontinuidades desaparecen al aumentar el amortiguamiento y para valores suficientemente altos del mismo (curvas inferiores) las curvas son continuas.

En general encontramos tres tipos de curvas. En un primer caso (figuras para $\Gamma=0.001$ por ejemplo) al alcanzarse $i_{d e p}^{0}$ el sistema salta de la rama superconductora $(v=0)$ a la resistiva óhmica $(v=i)$. En un segundo caso (curva para $\lambda=0.4$ y $\Gamma=0.1$ por ejemplo), en $i_{d e p}^{0}$ el sistema salta a una rama intermedia marcada por varios peldaños con discontinuidades entre los mismos. Esta rama es estable hasta valores relativamente altos de la corriente. Aumentando la corriente podemos definir una nueva corriente crítica, $i_{s w}^{0}$ que marca el salto desde esta nueva rama a la rama óhmica. En el tercer caso el amortiguamiento es grande y la curva $i$ - $v$ es suave, sin discontinuidades, pudiéndose apreciar una zona entre $i_{d e p}^{0}$ e $i \simeq 1$ donde el movimiento se corresponde con el movimiento localizado del fluxón en el anillo. Al aumentar la corriente el fluxón se deslocaliza y la curva se aproxima al límite óhmico de la misma.

La rama intermedia que aparece para valores moderados del amortiguamiento y valores medios y altos de $\lambda$ se corresponde con el movimiento coherente del fluxón en el anillo sin perder su formar y sin excitar la dinámica completa del sistema. La imagen correspondiente es la de una partícula dando vueltas en el anillo. La velocidad de movimiento del fluxón introduce una frecuencia en el sistema que se acopla, resuena, con los modos propios del sistema cuyo origen es la discretitud del mismo. De este modo los peldaños observados corresponden a resonancias entre ambas frecuencias características y el número de peldaños observables depende de los modos lineales accesibles que a su vez dependen del tamaño del sistema. Dicho fenómeno de resonancia ha sido ampliamente estudiado teórica y experimentalmente en el pasado [41, 42, 91, 92, 93, 95] En estos escalones la velocidad del fluxón permanece constante y la energía introducida en el sistema al aumentar la corriente se disipa aumentando la amplitud de las componentes ac de la dinámica sin cambiar el voltaje medio. Bajo condiciones de frontera periódicas, ocurre una resonancia de número $m$ cuando el paso del fluxón por un punto dado del arreglo, coincide con el $m$ máximo de oscilaciones inducidas por las ondas de plasma, lo que significa que la longitud de la unión debería acomodar $m$ longitudes de onda de la onda de plasma. De ese modo se muestra cuantitativamente las condiciones de resonancia para los anillos Josephson y encuentran un expresión en términos del voltaje:

$$
\frac{V}{V_{0}}=\frac{\Gamma}{m}\left[\frac{1}{\lambda}+4 \sin ^{2}\left(\frac{m \pi}{N}\right)\right]^{1 / 2}
$$



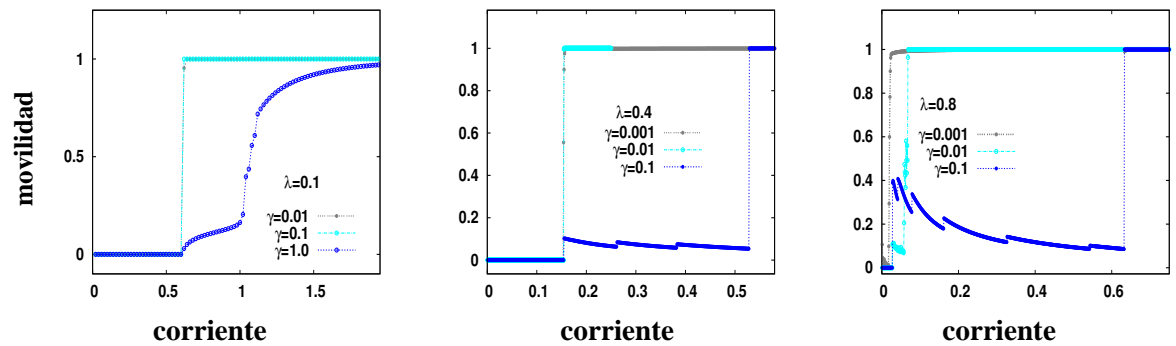

Figura 4.5: Movilidad del fluxón en un arreglo de 9 uniones para diferentes valores del amortiguamiento a temperatura cero y tres valores del acoplamiento; a la izquierda $\lambda=0.1$, en el centro $\lambda=0.4$ y a la derecha $\lambda=0.8$.
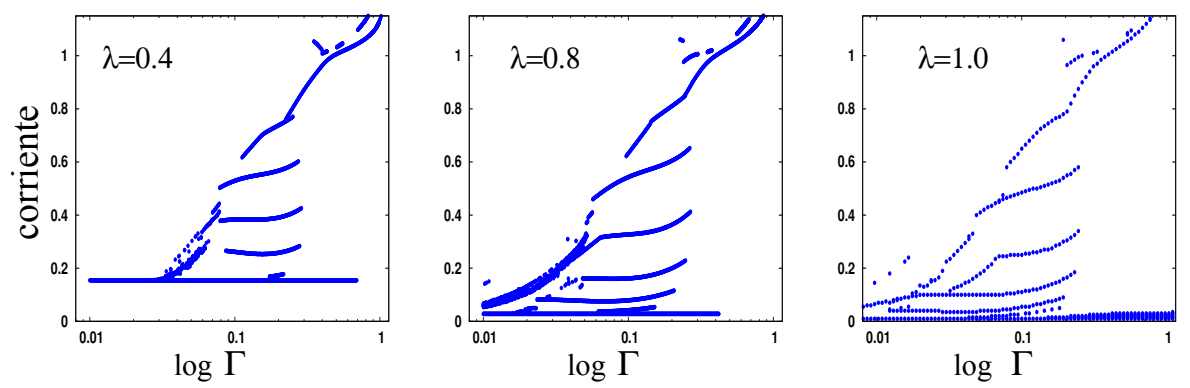

Figura 4.6: Diagrama de fases dinámico $i-\Gamma$ mostrando puntos donde la curve $i-v$ cambia de manera importante. Se observa claramente el depinning, las resonancias y el paso al modo resisitivo óhmico.

Esta es la formula derivada por Ustinov et al. [41] y da cuenta de las observaciones numéricas de los escalones en la región de bajo voltaje.

Otra imagen de lo que ocurre, se obtiene de los dibujos de la movilidad, definida como $v / i$, frente a la corriente. Así, por ejemplo, la figura 4.5 muestra este parámetro para valores pequeños del amortiguamiento y tres del acoplamiento. En estas figuras es más evidente los pasos resonantes por los que atraviesa la dinámica del sistema para el caso de amortiguamientos; $\Gamma=0.1$ y $\Gamma=0.01$.

Por último, la figura 4.6 intenta plasmar de manera global el diagrama de fases dinámico del sistema donde se representan los valores de la corriente para los cuales se observa un salto mayor de cierto umbral en la curva $i$ - $v$. A pesar de sus imperfecciones numéricas es fácil distinguir la zona central dominada por distintas resonancias y que separan la zona de amortiguamiento débil (curva discontinua con dos ramas: la superconductora y la óhmica) y la de amortiguamiento alto (curva continua).

Todas las curvas presentadas hasta el momento se refieren a las obtenidas al aumentar la corriente desde 0 hasta un determinado valor. No obstante 

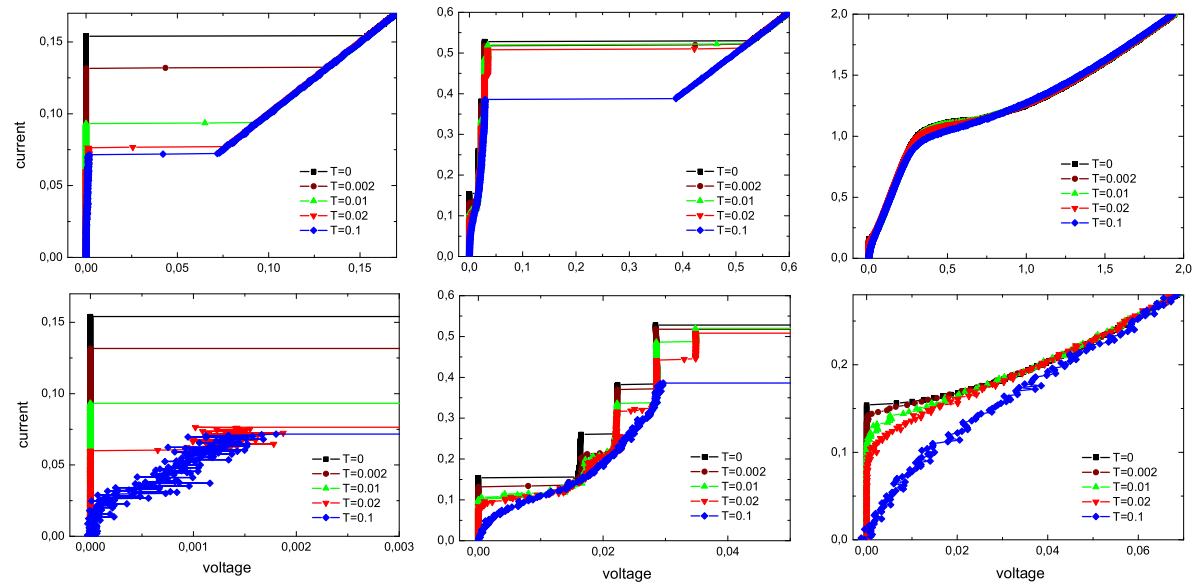

Figura 4.7: Curvas $i$ - $v$ para un fluxón en un anillo de 9 uniones Josephson con $\lambda=0.4$ a $\Gamma=0.01$ (izquierda), $\Gamma=0.1$ (medio) y $\Gamma=1.0$ (derecha). Cada curva muestra 5 temperaturas diferentes $(T=0,0.002,0.001,0.02,0.1)$.

también es posible trazar la curva $i-v$ disminuyendo la corriente desde un valor dado hasta cero. Para valores pequeños del amortiguamiento se encuentra que las curvas presentan fenómenos de histéresis y no reproducen de bajada el mismo camino seguido al aumentar la corriente. En particular los rangos de extensión de las diferentes ramas son distintos, las corrientes críticas también y el retorno a la rama superconductora, el llamado retrapping se produce a valores de la corriente sensiblemente menores que la corriente de desanclaje. Estos hechos ponen de manifiesto la existencia de una metaestabilidad dinámica en el problema con diferentes estados dinámicos estructuralmente estables para un mismo conjunto de valores de los parámetros del sistema. Esta histéresis disminuye al aumentar el amortiguamiento y desaparece para valores suficientemente altos del mismo, donde la curva $i-v$ es única.

\subsubsection{Curvas $I-V$ : el efecto de la temperatura}

En la presente sección describiremos brevemente el efecto de la temperatura sobre las curvas $I-V$ del anillo. Los resultados fundamentales están ilustrados en la figura 4.7 donde se muestran resultados para la dinámica de un fluxón en una red de 9 uniones con $\lambda=0.4$. Se han escogido tres valores del amortiguamiento, correspondientes a los tres tipos de comportamiento descritos en la sección anterior: subamortiguado $(\Gamma=0.01)$, de amortiguamiento moderado $(\Gamma=0.1)$ y sobreamortiguado $(\Gamma=1)$. En cada caso se muestran resultados para 5 temperaturas diferentes, $T=0,0.002,0.01,0.02$ y 0.1 . La rampa utilizada es $\frac{8}{3} \times 10^{-7}$. Las curvas superiores muestran curvas $i$ - $v$ completas y las 

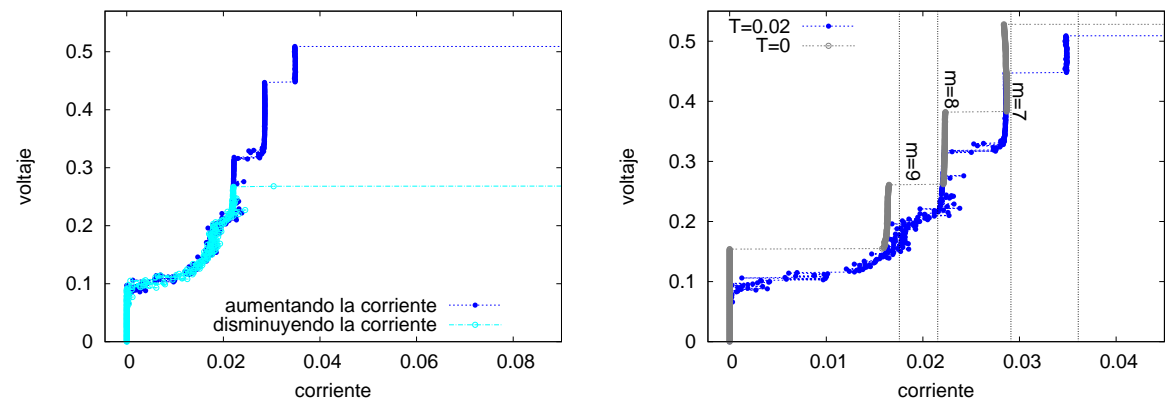

Figura 4.8: Escalones del fluxón en un arreglo de uniones Josephson con $\lambda=0.4$ y $\Gamma=0.1$. A la izquierda se muestra la serie de escalones en ambas direcciones de la corriente. A la derecha se aprecian los saltos con la corriente en aumento y los respectivos índices de resonancia de los escalones con temperatura 0.0 y 0.02 .

inferiores muestran la zona de bajo voltaje.

Es importante señalar que, contrariamente a como se ha hecho en figuras anteriores, la figura 4.7 utiliza el eje $x$ para representar el voltaje y el eje $y$ para la corriente. En principio esto podría parecer poco natural (ya que en simulaciones o experimento se varía la corriente $i$ y se mide el voltaje $v$, obteniéndose la curva $v(i))$ pero es el modo de representación utilizado habitualmente en el contexto de las redes de uniones Josephson y por ello hemos decidido utilizarlo a partir de aquí en el resto de las curvas $i$-v de la tesis.

Como se aprecia en la figura, en todos los casos se observa un decrecimiento de la corriente de depinning al aumentar la temperatura. Dicho efecto corresponde con el escape térmico del fluxón. El estudio de este comportamiento ha sido el objeto fundamental inicial de este trabajo de tesis doctoral y nos referiremos a él abundantemente más adelante. Además, puede observarse que a valores pequeños del amortiguamiento, si la temperatura es suficientemente alta, el ruido induce una rama de difusión de muy bajo voltaje del fluxón. La aparición de dicha rama: movimiento difusivo a bajo amortiguamiento, es un resultado inesperado y sorprendente y también será estudiado con cuidado más adelante.

Para amortiguamientos moderados la temperatura afecta tanto el depinning del fluxón como las transiciones entre los diferentes resonancias de la curva y el salto final a la rama resistiva óhmica. En particular un nivel de ruido suficientemente alto sustituye los escalones de la curva por una rama continua donde no es posible observar claramente el fenómeno de resonancia. La figura 4.8 muestra un detalle del efecto de la temperatura sobre las resonancias de la curva $I-V$. Se aprecia como el desanclaje es ruidoso, algunas resonancias se redondean hasta su desaparición y otras, las de mayor voltaje, siguen apre- 
ciándose claramente. El índice $m$ caracteriza cada resonancia según los valores predichos por la teoría (ecuación 4.2).

A amortiguamiento alto la curva $i$ - $v$ se suaviza notablemente en la región de bajo voltaje. En este último caso y también para amortiguamiento moderado y alta temperatura es difícil definir una corriente de desanclaje del sistema, ya que esta puede depender en gran medida del umbral de voltaje escogido para caracterizar el depinning. Por ello en nuestros estudios del efecto de la temperatura sobre el depinning del fluxón nos centraremos en la región de amortiguamiento pequeño.

\subsubsection{Desanclaje térmico del fluxón: $\left\langle i_{\mathrm{dep}}(T)\right\rangle$}

En esta sección vamos a presentar resultados sobre el desanclaje o depinning térmico de un fluxón en una red con nueve uniones [105]. La corriente de desanclaje es una variable estocástica con una distribución de probabilidad dada. En esta sección presentamos los resultados para el valor medio de la corriente de desanclaje $\left\langle i_{\mathrm{dep}}\right\rangle$ y su desviación estándar $\sigma$. Los resultados se obtuvieron a partir de la simulación numérica de las ecuaciones dinámicas del sistema para 1000 muestras y 10000 muestras. Presentamos diferentes valores del amortiguamiento $\Gamma$ (típicamente desde 0.001 a 0.1 ) y acoplamientos $\lambda$ (usualmente desde 0.2 a 1.0). Otro parámetro importante de las simulaciones es la rampa de corriente; en nuestro caso está rampa cambia de una simulación a otra, sin embargo es del orden de $10^{-7}$.

Usualmente la corriente de desanclaje está experimentalmente definida como la corriente para la cual el voltaje medido está por encima de un cierto valor umbral. Seguimos la misma definición para nuestras simulaciones. Esta es una buena aproximación en el régimen de bajo amortiguamiento y baja temperatura donde como hemos visto el sistema cambia entre dos valores muy diferentes del voltaje para un valor de corriente umbral. Sin embargo la elección del voltaje umbral no es trivial. Un umbral pequeño puede dar problemas a temperaturas grandes donde las fluctuaciones del voltaje son también grandes. Un umbral grande no es una buena selección ya que ignora posibles estados de bajo-voltaje como en la difusión del fluxón. A modo de ejercicio se puede observar la figura 4.3 e intentar situar valores adecuados de voltaje umbral en la misma. Por otro lado, si los estados de bajo-voltaje están presentes distinguimos entre dos corrientes críticas en el sistema: la corriente de desanclaje $i_{\text {dep }}$ y la corriente de switching $i_{\mathrm{sw}}$; donde el desanclaje marca el abandono del estado superconductor y el switching la transición a la rama de alto-voltaje. A temperaturas de ruido muy altas el sistema puede alcanzar el nivel umbral y la primera de las corrientes críticas desaparece. Para estudiar esto, hemos 


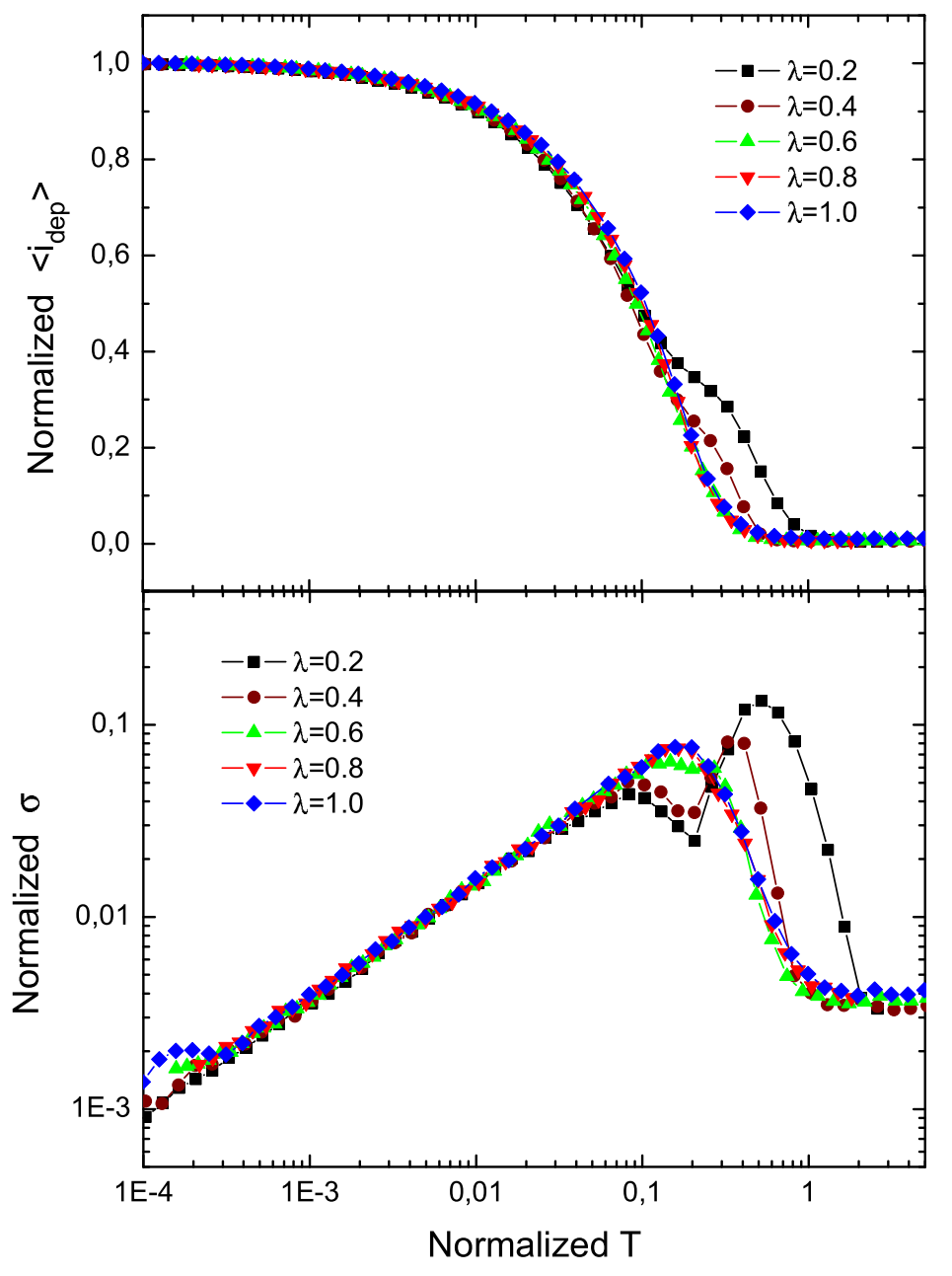

Figura 4.9: Cálculo numérico $\left\langle\widetilde{i}_{\text {dep }}\right\rangle$ y $\widetilde{\sigma}$ (corriente y desviación estándar normalizadas por $i_{d e p}^{0}$ ) contra $\widetilde{T}$ (temperatura normalizada por $E_{P N}$ ) a $\Gamma=0.01$ y diversos valores del acoplamiento $\lambda=0.2,0.4,0.6,0.8$ y 1.0 .

usado en nuestras simulaciones tres o cinco umbrales diferentes y comparamos los resultados entre ellos.

La figura 4.9 muestra resultados para $\Gamma=0.01$ y cinco diferentes valores del acoplamiento $\lambda=0.2,0.4,0.6,0.8$ y 1.0 . Como hemos visto a lo largo del capítulo 3, las principales propiedades físicas del fluxón en el arreglo cambian significativamente con el valor de $\lambda$. Así por ejemplo, la corriente de desanclaje a temperatura cero del arreglo $i_{\text {dep }}^{0}$ decrece un factor de 30 de $\lambda=0.2$ a $\lambda=1.0$. Para poder comparar las cinco curvas representadas, en la figura 4.9 ambos ejes han sido escalados por el valor de la corriente de desanclaje a temperatura cero para cada caso y la temperatura ha sido normalizada con altura de la 
barrera de potencial.

$$
\left.\widetilde{i}_{d e p}\right\rangle=\frac{\left\langle i_{d e p}\right\rangle}{i_{d e p}^{0}} \quad, \quad \widetilde{\sigma}=\frac{\sigma}{\left|i_{d e p}^{0}\right|} \quad y \quad \widetilde{T}=\frac{k_{B} T}{E_{P N}}
$$

La figura 4.9 muestra que una vez reescaladas las curvas aproximadamente colapsan en una significando que existe un marco común que permite entender los detalles fundamentales de todas ellas. Aquellos aspectos que se desvían de la curva de referencia común son los que se ven afectados de mayor manera por efectos de discretitud de la red dependientes del acoplamiento: ver fundamentalmente las desviaciones de las dos curvas de menor $\lambda$ en la zona de alta temperatura. En todos los casos se observa un decrecimiento paulatino de $\left\langle i_{\text {dep }}\right\rangle$ al aumentar la temperatura. Todas las curvas siguen el mismo comportamiento, pero a altas temperaturas en las curvas de $\lambda$ pequeña se observan pequeñas desviaciones con respecto a las otras. Ese régimen es complicado de estudiar, para temperaturas del orden de la barrera, las fluctuaciones térmicas dominan la dinámica y la corriente de desanclaje va a cero. En efecto, a altas temperaturas no existe una buena definición de la corriente de desanclaje ya que diferentes umbrales pueden dar diferentes resultados. Una estudio detallado de las distribuciones de probabilidad obtenidas muestra como a ciertos valores la distribución se vuelve bimodal, con una máximo centrado en valores pequeños de la corriente y otro en valores mayores, de ahí el aumento de $\sigma$ que se observa para $\lambda=0.2$ y $\lambda=0.4$ y temperaturas normalizadas cercanas a la unidad. Por otro lado en el régimen de baja temperatura la desviación estándar crece con $T^{2 / 3}$, de acuerdo con la teoría de activación térmica estándar que presentaremos más adelante, y alcanza un máximo a altas temperaturas, cuando $\left\langle i_{\text {dep }}\right\rangle \rightarrow 0$. Entonces la desviación estándar decrece ya que todos los eventos de escape suceden en un estrecho rango de valores de corriente.

\subsubsection{Comparación del escape del fluxón con el escape térmico de partícula en un potencial metaestable}

Tal y como hemos discutido a lo largo del tercer capítulo, cuando estudiamos la dinámica de un fluxón en el arreglo es muy común usar la imagen de partícula individual del mismo. Esta aproximación ha sido muy usada en el pasado y, como observaremos en lo que sigue, es muy útil aunque no siempre exacta. Una vez definida la coordenada centro de masas del fluxón, $X=C-\sum_{j} \varphi_{j}$ (ecuación 3.3), que indica básicamente la posición del fluxón en el arreglo, en la aproximación más simple, podemos considerar que la dinámica de un fluxón en un anillo se puede aproximar a la dinámica de una partícula masiva, forzada y amortiguada, que experimenta un potencial sustrato sinusoidal (potencial 
Cuadro 4.1: Parámetros del fluxón para dos valores de $\lambda$

\begin{tabular}{ccccc}
$\lambda$ & $E_{\mathrm{PN}}$ & $\omega_{\mathrm{PN}}$ & $m$ & $i_{\text {dep }}^{0}$ \\
\hline 0.4 & 0.30974 & 0.6691 & 0.3459 & 0.15435 \\
0.8 & 0.05539 & 0.3424 & 0.2363 & 0.02767
\end{tabular}

Peierls-Nabarro) y sujeta a fluctuaciones térmicas:

$$
m \ddot{X}+\Gamma m \dot{X}+i_{\mathrm{dep}}^{0} \sin X=i+\xi(\tau)
$$

donde

$$
\langle\xi(\tau)\rangle=0 \text { and }\left\langle\xi(\tau) \xi\left(\tau^{\prime}\right)\right\rangle=2 m \Gamma T \delta\left(\tau-\tau^{\prime}\right)
$$

En está simple aproximación estamos despreciando, por ejemplo, la dependencia espacial de la masa, el amortiguamiento efectivo debido a otros grados de libertad del sistema y términos de más alto orden en la expansión del potencial sustrato para el fluxón.

La tabla 4.1 muestra a modo de ejemplo una relación de los principales parámetros del fluxón computados numéricamente para dos valores distintos del acoplamiento $\lambda: E_{\mathrm{PN}}$ es la barrera de potencial a corriente cero; $\omega_{\mathrm{PN}}$ es la frecuencia característica de las oscilaciones de pequeña amplitud del fluxón alrededor del equilibrio; $m$ es la masa efectiva del fluxón en reposo (calculada como $\left.m=E_{\mathrm{PN}} / 2 \omega_{\mathrm{PN}}^{2}\right)$ y $i_{\text {dep }}^{0}$ la corriente de desanclaje. Si el potencial efectivo del fluxón fuese un potencial sinusoidal perfecto debería obtenerse $i_{\text {dep }}^{0}=E_{\mathrm{PN}} / 2$. El resultado obtenido es cercano a este valor.

Siguiendo la imagen de partícula individual en esta sección presentaremos resultados del estudio del escape térmico de una partícula integrando la ecuación 4.4 con los valores adecuados de $m$ e $i_{d e p}^{0}$ para cada valor de $\lambda$, y los compararemos con los obtenidos para el caso del fluxón. Por otro lado también presentaremos cálculos numéricos basados en los resultados analíticos de la teoría de Kramers y sus extensiones.

Aunque el capítulo 6 estudia con detalle el problema del escape térmico de partículas en el límite subamortiguado, presentaremos a continuación brevemente un rápido resumen de como hemos obtenidos los resultados basado en la teoría. Para calcular la corriente de desanclaje promedio necesitamos conocer la distribución de probabilidad $P(i)$ que nos indica la probabilidad de que un evento de desanclaje ocurra entre $i$ e $i+d i$ : $\langle i\rangle=\int i P(i) d i$. Tal distribución de probabilidad puede ser calculada a partir de las tasas de escape $r(i)$ que indican el ritmo promedio de escape de partículas situadas en el interior del pozo de potencial cuando se aplica una corriente constante $i$ [25]:

$$
P(i)=r(i)\left(\frac{d i}{d t}\right)^{-1}\left(1-\int_{0}^{i} P(u) d u\right) .
$$


Podemos ver que en dicha expresión aparece la rampa o tasa de cambio de $i$ en el experimento o en la simulación. Con respecto al cálculo de la tasa $r(i)$ existen diversas teorías que dan aproximadamente el valor de dicha función en el régimen de amortiguamiento pequeño-moderado. En particular hemos realizado comparaciones utilizando la teoría de Kramers [27] para muy bajo amortiguamiento, y las extensiones de Büttiker et al. (teoría BHL) [36] y de Melnikov et. al [96] para el régimen de amortiguamiento bajo-moderado. De entre ellas vemos que los resultados de la teoría BHL son suficientes para entender la mayoría de nuestras simulaciones y resultan prácticamente indistinguibles de los obtenidos utilizando la teoría de Melnikov.

Según la teoría BHL:

$$
r(i)=k \frac{\omega_{a}}{2 \pi} \mathrm{e}^{-\Delta U / k_{B} T}
$$

donde

$$
k=\frac{4}{\left[\left(1+\omega_{a} k_{B} T / 1.8 \Gamma \Delta U\right)^{1 / 2}+1\right]^{2}}
$$

y si definimos $f=i / i_{\text {dep }}$

$$
\Delta U(i)=E_{P N}\left[\left(1-f^{2}\right)^{1 / 2}-f \arccos f\right]
$$

y

$$
\omega_{a}(i)=\omega_{P N}\left(1-f^{2}\right)^{1 / 4} .
$$

Entonces contamos con todos los ingredientes necesarios para evaluar numéricamente la distribución de probabilidad $P(i)$ y a partir de ella el valor de $\langle i\rangle$ y comparar el resultado obtenido con las simulaciones realizadas para una partícula y para un fluxón.

La figura 4.10 muestra el resultado para $\lambda=0.4$ (izquierda) y $\lambda=0.8$ (derecha) y amortiguamiento $\Gamma=0.01$, en ambos casos se realiza una comparación entre los resultados para el fluxón en el arreglo, las simulaciones numéricas del desanclaje de una partícula en un potencial sinusoidal (ecuación 4.4) y el cálculo teórico basado en los resultados analíticos para la tasa de activación térmica de partículas en potenciales sinusoidales en el régimen de bajo amortiguamiento. En general la comparación entre las tres curvas es excelente y en particular las simulaciones de la partícula individual reproducen los resultados para el fluxón de forma excelente a este valor del amortiguamiento y para todo el rango de temperatura aunque se observa una pequeña desviación, señalada con un circulo punteado, en una parte del rango de temperaturas para $\lambda=0.4$. Por otro lado el acuerdo entre el resultado teórico y las simulaciones de partícula individual es algo en gran medida esperado.

Sin embargo, el acuerdo mostrado en las figuras 4.10 no ocurre a otros valores del acoplamiento y amortiguamiento. Por ejemplo, para $\lambda=0.4$ y $\Gamma=0.001$ 

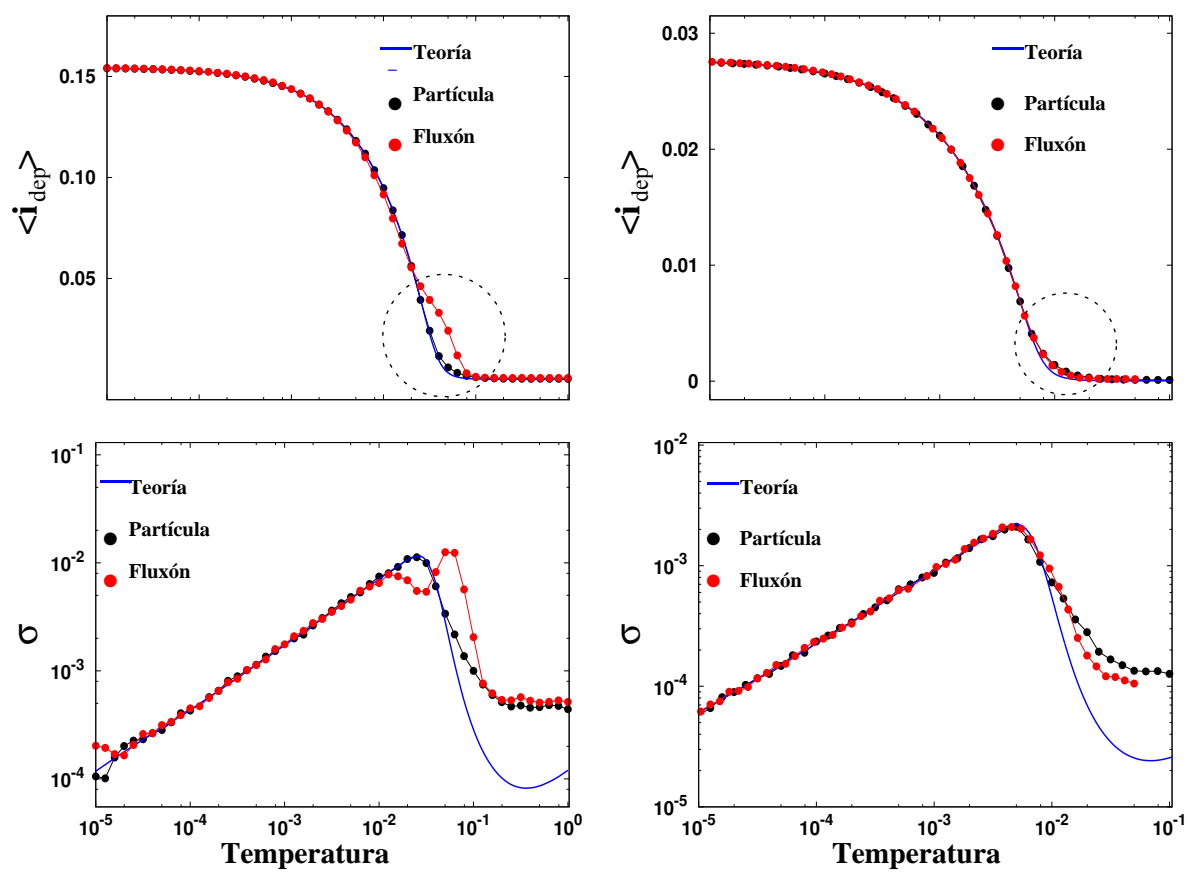

Figura 4.10: $\left\langle i_{\text {dep }}\right\rangle$ y $\sigma$ versus $T$ a $\Gamma=0.01$ y $\lambda=0.4$ (izquierda) y 0.8 (derecha) para el fluxón y una partícula aislada en un potencial periódico y la comparación con la predicción teórica.

(figura 4.19 más adelante) se ha observado una desviación de la curva simulada con respecto a la predicción teórica.

A continuación mostraremos un estudio de los resultados en función del amortiguamiento $\Gamma$ para un valor determinado de acoplamiento y temperatura; en particular, $\lambda=0.4(T=0.01)$ y $\lambda=0.8(T=0.0018)$. Se han elegido dos temperaturas distintas para obetenr en cada caso un mismo valor del cociente $T / i_{\text {dep }}^{0}$. Los resultados se muestran en las figuras 4.11 y 4.12 . Vemos que en el caso de $\lambda=0.4$ los resultados del fluxón se desvían significativamente de lo esperado para partícula única y la teoría cuando el amortiguamiento decrece. Así para $\Gamma=10^{-5}$ la corriente de escape teórica es de 0.1318 , la simulada para la partícula es de 0.1374 y la obtenida para el caso del fluxón es tan sólo 0.111. Esto representa una reducción de un $16 \%$ respecto el resultado teórico y y de un $20 \%$ respecto la simulación de partícula individual. Sin embargo este no es el caso para $\lambda=0.8$ en donde las diferencias apenas son notorias entre el fluxón y la simulación de la partícula en todo el rango de amortiguamiento considerado. Sin embargo para amortiguamiento pequeño podemos notar una diferencia entre las simulaciones numéricas y los cálculos basados en los resultados de la teoría. Esta diferencia de comportamiento entre los resultados para 


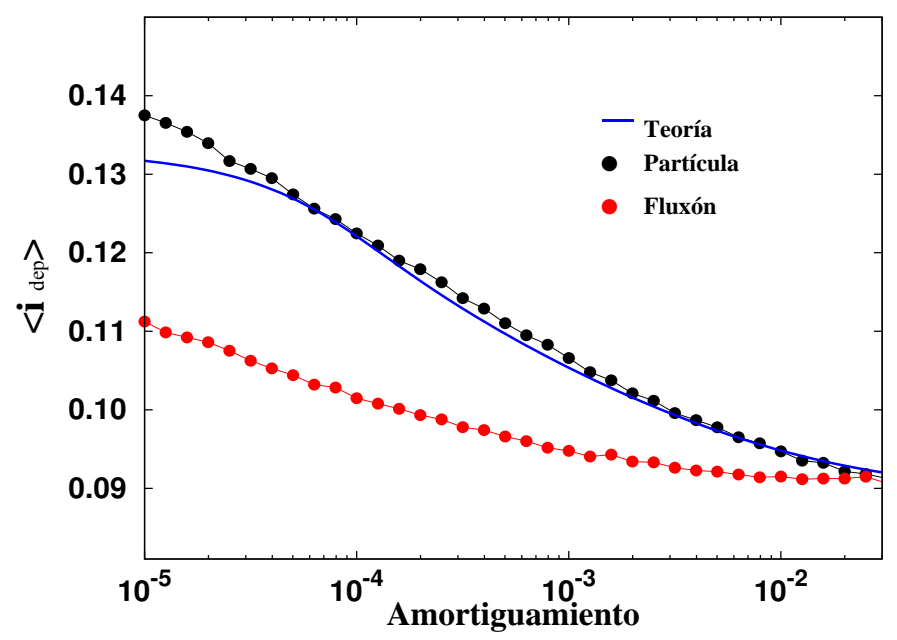

Figura 4.11: $\left\langle i_{\mathrm{dep}}\right\rangle$ como función del amortiguamiento $\Gamma$ con acoplamiento $\lambda=0.4$ a $T=0.01$.

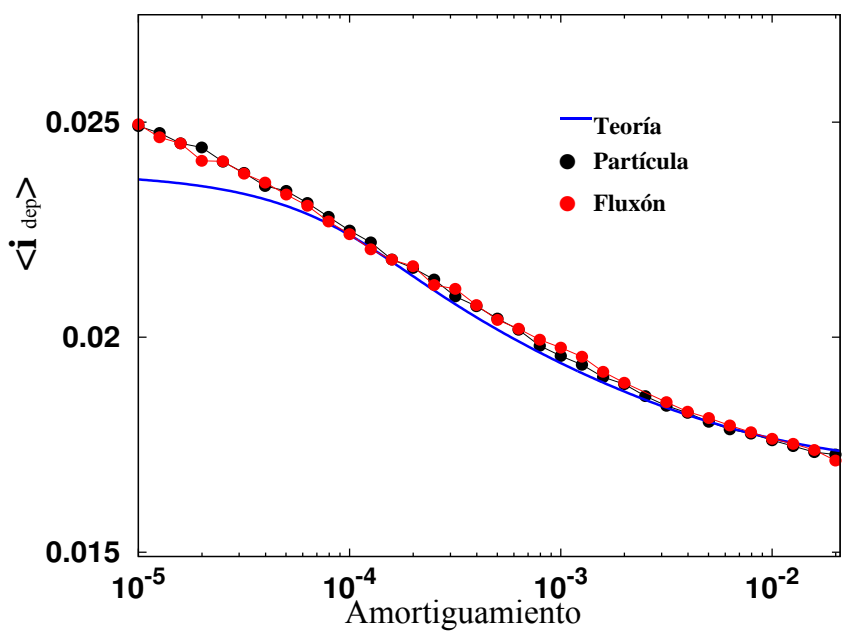

Figura 4.12: $\left\langle i_{\text {dep }}\right\rangle$ como función del amortiguamiento $\Gamma$ con acoplamiento $\lambda=0.8$ a $T=0.0018$.

los dos valores de $\lambda$ presentados pone en evidencia la importancia del grado de discretitud del sistema. Tal grado es medido por el parámetro de acoplamiento $\lambda$ (alto $\lambda$ se aproxima al límite continuo del sistema, y un pequeño $\lambda$ incrementa los efectos de discretitud). A pequeños valores de $\lambda$ los efectos debidos a otros grados de libertad son más importantes y si el amortiguamiento es pequeño tales excitaciones persisten significativamente en el sistema. Entonces la imagen de partícula individual falla de manera importante. 


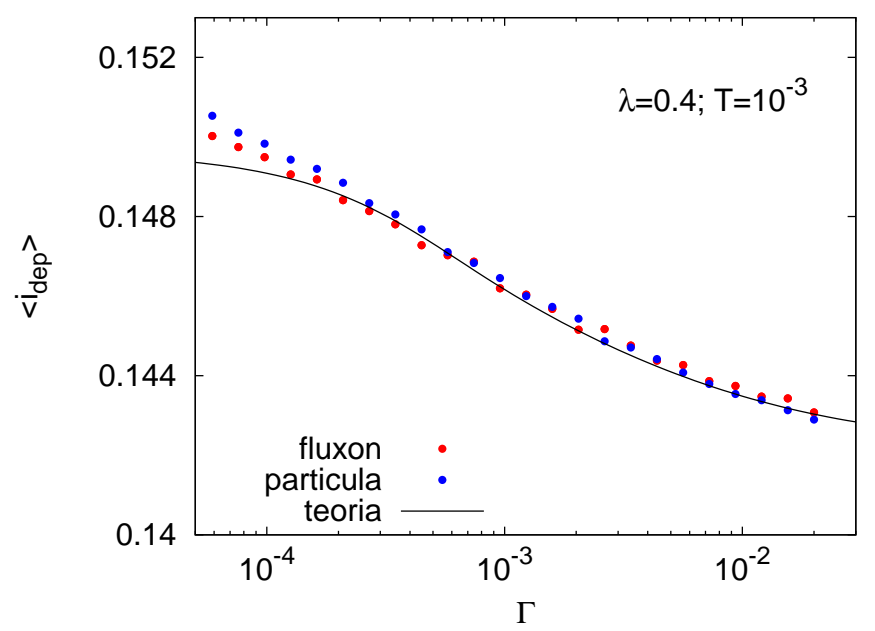

Figura 4.13: $\left\langle i_{\text {dep }}\right\rangle$ como función del amortiguamiento $\Gamma$ con acoplamiento $\lambda=0.4$ a $T=0.001$.

Además, en el caso de $\lambda=0.4$ mostramos la simulación numérica a temperatura ( $T=0.001$ ), figura 4.13. A este valor de la temperatura, como vemos, no aparece una diferencia significativa entre la teoría y la simulación.

Uno de los aspectos más sobresalientes por inesperados, puestos en evidencia en las figuras 4.11 y 4.12 es la falta de acuerdo entre los resultados teóricos y las simulaciones de una partícula en el régimen de amortiguamiento muy pequeño. Esta discrepancia es importante puesto que en principio los resultados teóricos empleados son muy precisos a amortiguamiento bajo. Si esto es así entonces la diferencia estaría denotando una importante inexactitud en nuestras simulaciones en el régimen de amortiguamiento muy pequeño. Una serie de cuidadosas simulaciones adicionales nos permitieron descartar esta última posibilidad. Por lo tanto, volviendo a atrás en el razonamiento, las curvas dibujadas ponen de manifiesto una imprecisión en los resultados teóricos utilizados (los utilizados habitualmente en multitud de trabajos previos por muchos autores) en nuestro cálculo. Para resolver este inesperado problema, decidimos variar los planes inicialmente previstos de progreso del trabajo de tesis doctoral y nos internamos en el estudio de la teoría de escape de Kramers para valores muy pequeños del amortiguamiento del sistema. Esta labor consumió una parte importante del trabajo de tesis y los resultados obtenidos se presentan en el capítulo 6 de esta memoria. 


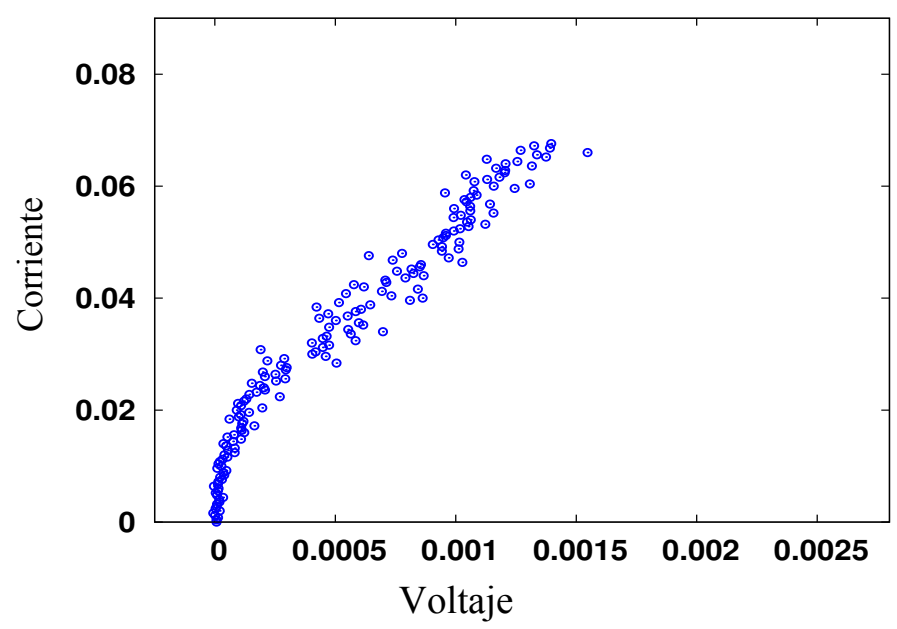

Figura 4.14: Curva $i$ - $v$ mostrando la rama de difusión del fluxón a voltaje bajo obtenida para $\lambda=0.4, \Gamma=0.01$ y $T=0.1$.

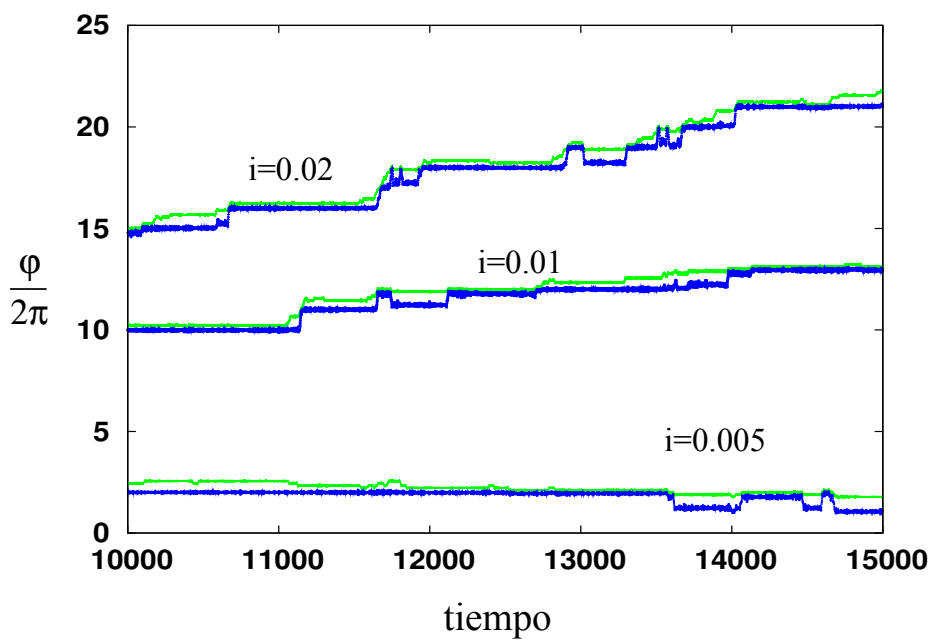

Figura 4.15: Sistema con $\lambda=0.4, \Gamma=0.01$ and $T=0.1$. Evolución temporal de la fase de la 1 unión (línea verde) y la fase del centro de masas del fluxón (línea azul) dividida por $2 \pi$ a $i=0.005,0.01,0.02$.

\subsubsection{Difusión del Fluxón}

En la figura 4.7 hemos visto que a bajo amortiguamiento y temperatura suficientemente alta aparece una rama de bajo voltaje en las curvas $I$ - $V$ previa al escape al estado óhmico. En este estado de bajo voltaje, el transporte de los fluxones ocurre a través de una serie de cambios de fase de magnitud $2 \pi$ inducidos por ruido (entonces el centro de masas del fluxón cambia en $2 \pi / N$ ). 


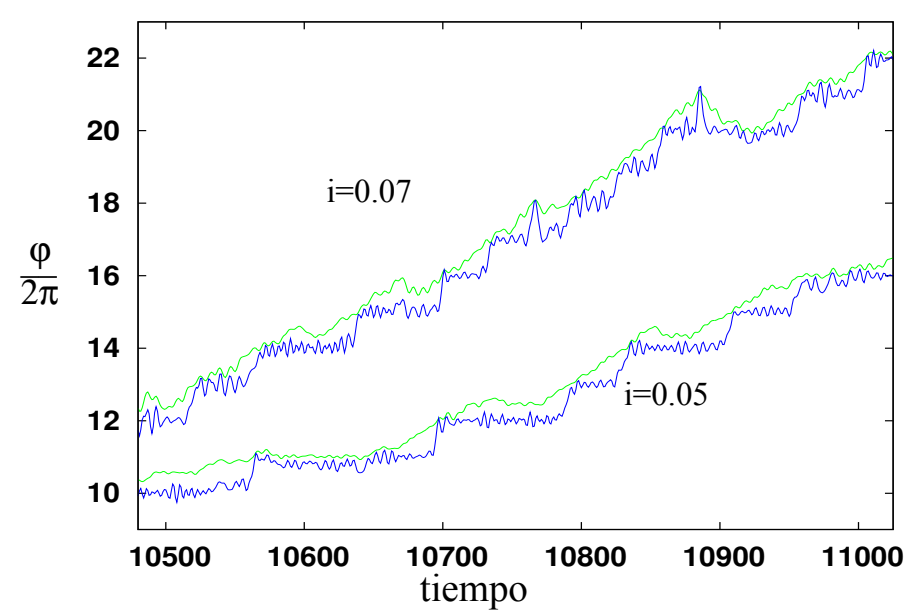

Figura 4.16: Sistema de uniones con $\lambda=0.4, \Gamma=0.01$ and $T=0.1$. Evolución temporal de la fase de la 1 unión (línea verde) y la fase del centro de masas del fluxón (línea azul) dividida por $2 \pi$ a $i=0.05$ y 0.07 .

Cada salto se corresponde con un avance por parte del fluxón de una celda en el arreglo superconductor. Tal estado no puede ser entendido en términos de la imagen de partícula única, ecuación 4.4. En analogía con la difusión de fase que ocurre en una sola unión a valores altos del amortiguamiento, denominamos a este modo de transporte difusión del fluxón. También podemos apreciar este fenómeno en la figura 4.14 para un sistema de 9 uniones y acoplamiento de 0.4, en donde hemos explorado la física en el estado de bajo voltaje, previo al switching. A pesar del hecho de estar en el régimen de bajo amortiguamiento, el fluxón es capaz de viajar a lo largo del anillo sin excitarse a la rama whirling. Sorprendentemente este es un estado excitado térmicamente que no se observa a bajas temperaturas.

En la figura 4.15 y figura 4.16 mostramos la evolución temporal de la fase de una unión (unión 1) y la fase asociada con el centro de masa del fluxón definido como $\psi=\frac{1}{N} \sum_{j} \varphi_{j}$ para permitir una mejor comparación. Los valores de la corriente han sido escogidos en la rama de bajo voltaje. Los saltos aleatorios de magnitud $2 \pi$ para la unión ó $2 \pi / N$ para la fase del fluxón $\psi$ se observan fácilmente. Tales saltos son excitados térmicamente y son más frecuentes a valores mayores de la corriente (la energía de excitación de los mismos en menor). Para valores pequeños de la corriente también es posible observar saltos hacia atrás del fluxón. A estas temperaturas el acoplamiento del sistema al resto de grados de libertad es grande y el fluxón disipa rápidamente la energía extra adquirida en cada salto evitándose la transición a la rama óhmica.

El régimen de difusión, tambien se observa en valores mayores del acopla- 


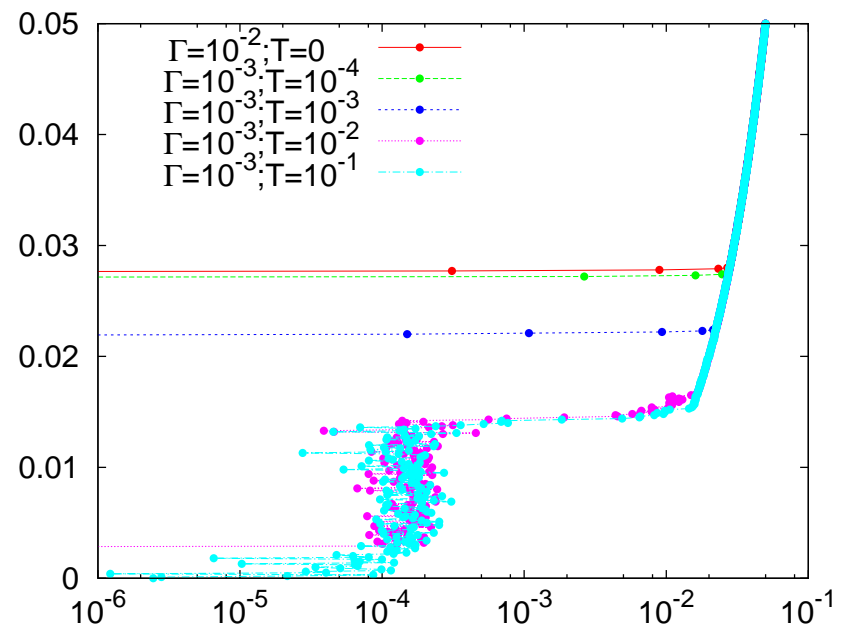

Figura 4.17: Curva $i-v$ que muestra a alta temperatura la rama de difusión del fluxón a voltaje bajo obtenida para $\lambda=0.8, \Gamma=0.001$

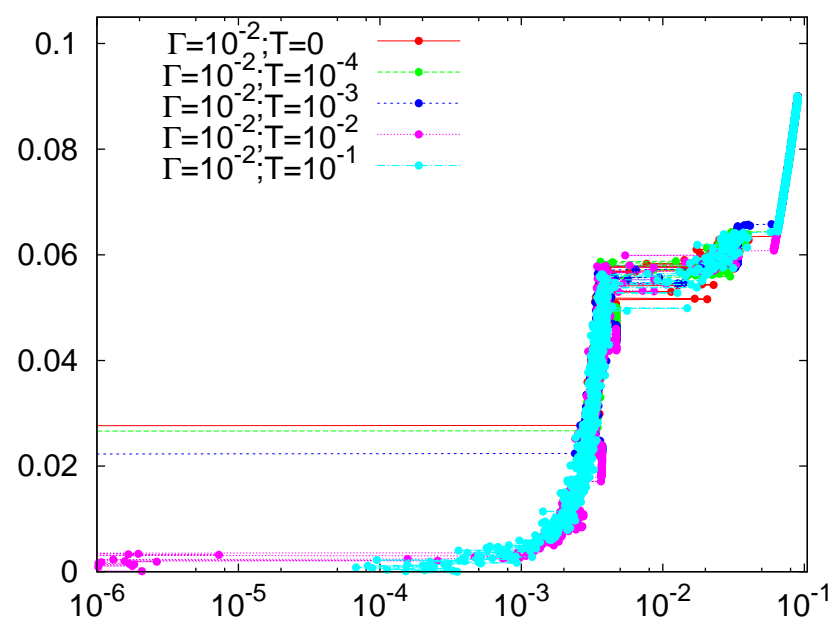

Figura 4.18: Curva $i-v$ que muestra a alta temperatura la rama de difusión del fluxón a voltaje bajo obtenida para $\lambda=0.8, \Gamma=0.01$

miento entre las uniones, como en el caso de $\lambda=0.8$ dibujado en la figura 4.17 con amortiguamiento $\Gamma=0.001$ y la figura 4.18 con amortiguamiento $\Gamma=0.01$ en donde se muestra que a altas temperaturas aparece una zona de bajo voltaje previo al salto.

Hemos realizado también una serie de experimentos numéricos para estudiar el salto desde la rama de difusión al estado resistivo óhmico de alto voltaje o whirling mode en el que todas las fases del sistema cambian y el fluxón se des- 
localiza en la red. Estas simulaciones se hacen eligiendo un umbral de voltaje mayor, pero son, de otra forma similares a las simulaciones descritas previamente. Los resultados para $\Gamma=0.01$ y 0.001 se muestran en la figura 4.19 para $\lambda=0.4$. Vemos que la teoría y los resultados para partícula única están completamente de acuerdo en todo el rango de temperatura. También podemos ver que la desviación estándar se incrementa con la temperatura siguiendo la ley esperada, hasta un cierto valor donde alcanza un máximo y entonces decrece cuando $\left\langle i_{\text {dep }}\right\rangle$ es cercana a cero.

Sin embargo, en la figura 4.19 podemos también ver que para la curva del fluxón existe un valor de $T$ a partir del cual para temperaturas mayores se observa que el valor de la corriente para el cual el sistema salta al estado óhmico, $i_{s w}$ es básicamente independiente de la temperatura. Esta temperatura muestra la emergencia de la rama de difusión del fluxón en la curva $I$ - $V$ y es más notoria en la medida que aumenta el valor del amortiguamiento. Al observar las curvas $I-V$ parece observarse que existe una velocidad máxima para el fluxón en el sistema que define aproximadamente el valor de la corriente para el cual se produce el evento de salto a la rama óhmica. Comparando la curva de la desviación estándar de las figura 4.19 y 4.10, podemos ver que el máximo en la $i_{s w}$ del fluxón ocurre a temperaturas menores que el máximo en la $i_{d e p}$. Dicho máximo marca la temperatura a la cual comienza a observarse la rama de difusión y la anchura de la distribución de probabilidad asociada en mucho menor y además se mantiene constante al aumentar la temperatura.

En la imagen de una partícula, o el modelo RCSJ para una unión, generalmente se acepta que la difusión no puede coexistir con la histéresis o dinámica subamortiguada. Esto fue bien dilucidado por Kautz y Martinis [97] utilizando argumentos del espacio de fase. En pocas palabras, si el valor de la corriente aplicada es suficiente para permitir un estado running estable que coexiste con los puntos fijos de voltaje cero, las cuencas de atracción para tal estado running necesariamente separan las cuencas de atracción de cualquiera dos puntos fijos vecinos. Los saltos de la fase entre dos puntos fijos están prohibidos, ya que el sistema debe pasar a través de la cuenca de atracción por el estado running. Aunque hemos demostrado en este capítulo, que el escape inicial del fluxón desde su mínimo se puede explicar por el fenómeno de activación térmica de una partícula, el estado de difusión del fluxón en las curvas $I$ - $V$ de la figura 4.7 y la figura 4.14 no puede ser explicado de manera similar. A pesar de lo dicho, es posible encontrar una serie de experimentos $[98,99,100]$ donde al estudiar la dinámica de una unión Josephson con amortiguamiento pequeño se observó una rama de difusión de voltaje pequeño. En este caso, la coexistencia de difusión de fase e histéresis fue explicada por la presencia del amortiguamiento dependiente de la frecuencia en la carga de la unión. Un modelo simple de amortiguamiento dependiente de la frecuencia es un circuito serie RC en 

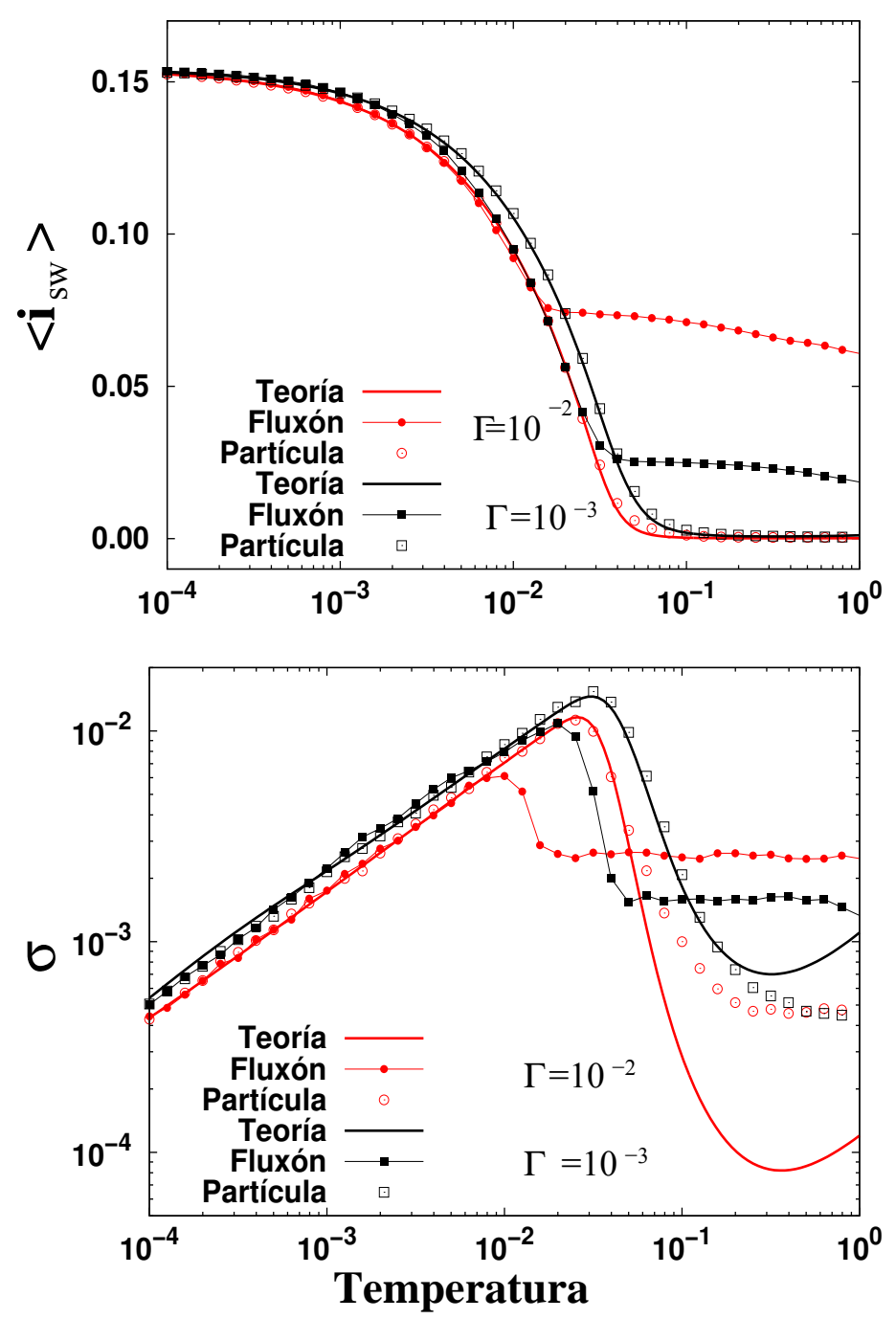

Figura 4.19: $\left\langle i_{s w}\right\rangle$ y $\sigma$ como función de $T$ a $\lambda=0.4$, para dos valores diferentes del amortiguamiento $\Gamma=0.01$ y $\Gamma=0.001$. Mostramos las gráficas para la predicción teórica (líneas), la partícula (símbolos huecos) y el fluxón (símbolos rellenos).

paralelo con la unión, el cual adiciona un grado extra de libertad al espacio de fase para la dinámica de la unión. Está dimensión extra resuelve el tema antes mencionado respecto a las cuencas no sobrepuestas de atracción. Un aspecto relevante en los resultados es que se observa la difusión del fluxón en las simulaciones sin que el amortiguamiento dependa de la frecuencia, lo cual no se incluyó en las ecuaciones. En lugar de las dimensiones extra introducidas por el amortiguamiento dependiente de la frecuencia, la difusión del fluxón puede ocurrir por los grados de libertad adicionales de las múltiples uniones 


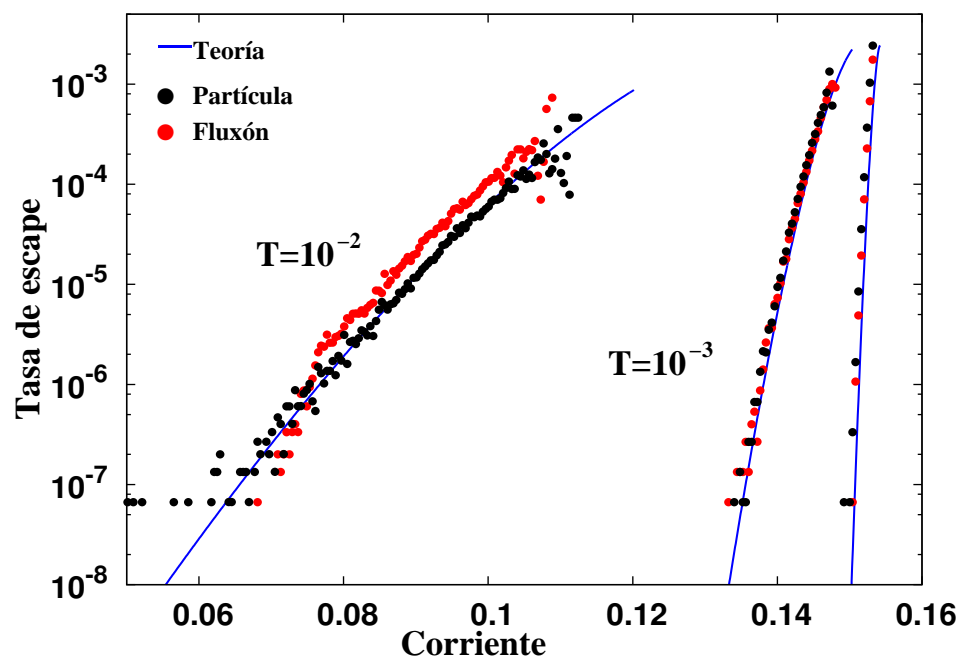

Figura 4.20: Tasa de escape como función de la corriente a 3 valores de temperatura $\left(10^{-2}, 10^{-3}\right.$ y $\left.10^{-4}\right)$ para $\lambda=0.4$ y $\Gamma=0.01$

en el arreglo. Cuando estudiamos el comportamiento del sistema a diferentes temperaturas hemos visto que el valor medio de la distribución de la corriente de switching de la rama de difusión del fluxón y la desviación estándar es muy pequeña. Comparando la desviación estándar de la curva en la figura 4.19 con la de más bajo umbral en la figura 4.10, también vemos que el pico ocurre mucho más pronto en temperatura para el salto desde el estado de difusión. Este pico en la desviación estándar es reminiscencia de los experimentos de una unión [98, 99, 100], donde un pico en la desviación estándar indica el colapso de la activación térmica y el comienzo de la difusión.

\subsubsection{Tasas de escape}

Hasta ahora hemos presentado resultados para el valor medio y la desviación estándar de la distribución de probabilidad de corriente del escape del fluxón. También es posible describir los datos en términos de la tasa de escape, la cual es una magnitud que no depende de detalles experimentales tal como la rampa de la corriente. La tasa de escape se puede calcular fácilmente desde la función distribución de probabilidad [25] aunque en general se requiere una estadística excelente para obtener resultados satisfactorios. Para un número limitado de valores de temperatura hemos calculado los valores de la corriente de desanclaje para 10000 muestras y extraído de los resultados los valores de la tasa de escape.

Los resultados del cálculo de la tasa de escape a partir de la función de 


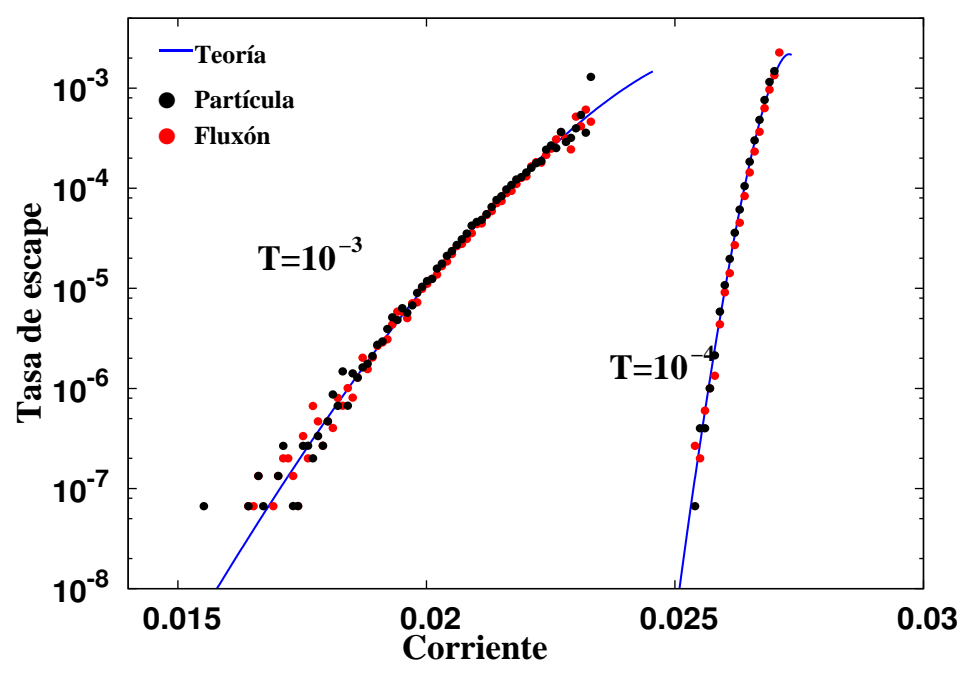

Figura 4.21: Tasa de escape como una función de la corriente a dos diferentes temperaturas $\left(10^{-3}\right.$ y $\left.10^{-4}\right)$ para $\lambda=0.8$ y $\Gamma=0.01$

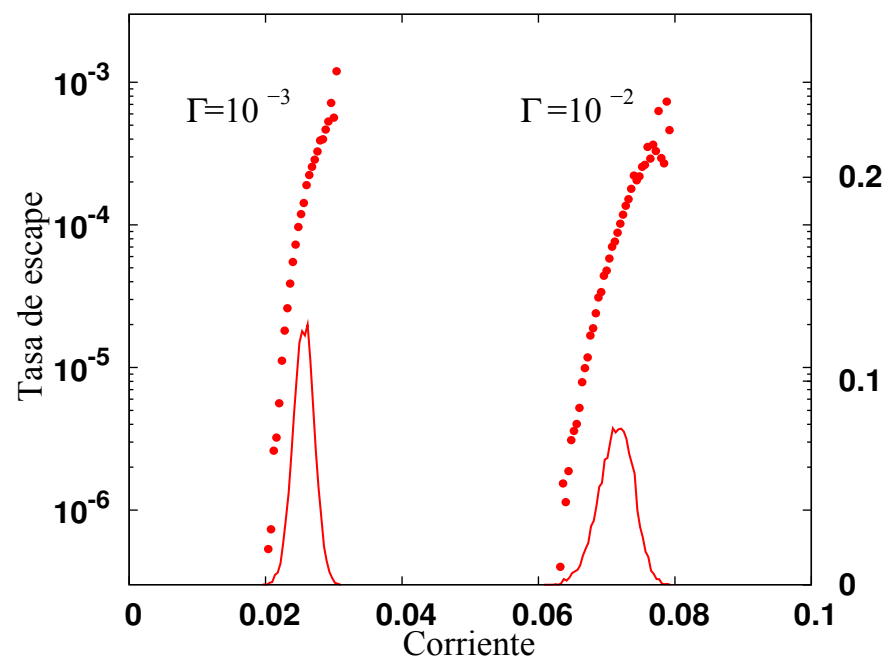

Figura 4.22: Tasa de escape y función de distribución de probabilidad para el switching de la rama de difusión del fluxón. $\lambda=0.4, \Gamma=0.01$ y 0.001 y $T=0.1$.

distribución de probabilidad se muestran en la figura 4.20 y figura 4.21. Como se esperaba de las figuras 4.10, el acuerdo entre los resultados numéricos para el fluxón, la partícula y la teoría son excelentes cuando el acoplamiento es de $\lambda=0.8$ y para $\lambda=0.4$ los resultados son comparables cuando la temperatura es baja. El fluxón se desvía ligeramente para $\lambda=0.4$ y $T=0.01$, cerca a la región con una rama de difusión del fluxón. 
Por otro lado también hemos calculado la función distribución de probabilidad y la tasa de escape asociada para el switching desde el estado de bajo voltaje difusión del fluxón al modo de alto voltaje, figura 4.22. Se ha elegido $T=0.1$ y $\Gamma=0.01$ y 0.001 (ver también figura 4.19 ). En este caso no tiene sentido comparar con los resultados para una partícula puesto la rama de difusión del fluxón no puede entenderse en términos del comportamiento de partículas individuales.

\subsection{Red regular de $30,60,120$ y 300 uniones}

En esta sección presentaremos un análisis de la dinámica del fluxón en anillos con un número mayor de uniones. En particular estudiaremos los casos de anillos con 30, 60, 120 y en algunos casos 300 uniones Josephson. Centraremos nuestro interés en la misma región de parámetros que en la sección anterior; esto es, sistemas discretos y subamortiguados (acoplamiento de $\lambda=0.4$, y amortiguamientos de $\Gamma=0.001, \Gamma=0.01$ y $\Gamma=0.1$ ). Nos interesaremos en el comportamiento del sistema a distintas temperaturas. Comenzaremos presentando los resultados de la dinámica a partir de las curvas de corriente-voltaje, centrándonos especialmente en el movimiento del fluxón en la zona de difusión a bajo amortiguamiento. Posteriormente estudiaremos el efecto de la temperatura sobre las diferentes corrientes críticas del sistema, fundamentalmente la que hemos llamado corriente de switching que marca la transición a la rama resistiva óhmica.

En todos los casos la rama de $V=0$ corresponde al fluxón anclado en la red y la rama óhmica corresponde a todas las uniones rotando con a la misma frecuencia y con un desfase $2 \pi / N$. La corriente de desanclaje es independiente del tamaño de la red. Sin embargo, la rama de bajo voltaje y la corriente de switching van a depender del tamaño de la red. En cualquier caso, el voltaje en esa zona es debido al movimiento del fluxón a lo largo del anillo mientras las uniones más alejadas al centro de masas de éste se encuentran aproximadamente en reposo o oscilando en torno a su posición de equilibrio (según el valor de $N$, la velocidad del fluxón y el amortiguamiento). La velocidad del fluxón corresponde a $v_{\text {fluxon }}=N \times v$. El fluxón, en su movimiento circular a lo largo del anillo excita las uniones que se encuentra en su paso. Si el amortiguamiento es grande o el anillo muy grande, las uniones a las que se aproxima están en un estado de reposo, entonces la dinámica es independiente de $N$ y el movimiento en redes de distinto tamaño es equivalente. Sin embargo, para redes pequeñas o valores del amortiguamiento pequeños (ambas cosas se dan en los casos que nos interesan) el fluxón a su paso se encuentra con uniones que no han llegado al equilibrio y que fueron excitadas por el paso del fluxón 
en la vuelta anterior. En este caso los efectos de interacción entre el fluxón y los modos excitados son fundamentales, produciéndose las resonancias de la curva $I-V$. En ese caso la dinámica depende de manera importante del tamaño de la red y en particular se observa como la corriente de switching cambia de manera importante con este tamaño. A continuación pasaremos a estudiar con más detalle y cuantificar estos efectos.

\subsubsection{Curvas $I-V$}

A continuación presentaremos resultados obtenidos en simulaciones de la dinámica del sistema tomando como referencia un anillo con acoplamiento moderadamente discreto, $\lambda=0.4$. Compararemos las curvas $I$ - $V$ de sistemas con $N=9,30,60$ y 120 uniones a tres valores distintos de la temperatura: $T=0$, $T=0.02$ y $T=0.1$. En todos los casos representados (ver figuras $4.23,4.24$ y 4.25 ) se dibuja la curva $i$ - $v$ convencional (gráficas situadas en la columna izquierda de la figura) y el detalle de la región de bajo voltaje (gráficas situadas en la columna central de la figura). Además, dado que la región de bajo voltaje está dominada por la dinámica del fluxón, con el fin de poder comparar adecuadamente las curvas obtenidas para distintos valores del tamaño de red $N$ resulta conveniente dibujar también la contribución del movimiento del fluxón al voltaje dc del sistema. Dicha contribución es simplemente $v_{\text {fluxon }}=N \times v$. Las gráficas situadas en la columna derecha de las figuras mencionadas muestran el cambio de $v_{\text {fluxon }}$ con $i$ en la región de bajo voltaje.

Comenzaremos nuestro estudio presentando resultados para valores pequeños del amortiguamiento $\Gamma=0.001$ (figura 4.23). Nuestro objetivo al elegir dicho valor del parámetro de amortiguamiento del sistema es estudiar fundamentalmente como el tamaño del sistema afecta la emergencia de la rama de difusión observada a altas temperaturas. Las curvas de temperatura nula (mostradas en las gráficas de la fila superior), no muestran nada inesperado: la corriente de desanclaje del fluxón es básicamente independiente del tamaño de la red y al alcanzarse $\left(i_{\text {dep }} \simeq 0.154\right)$ el sistema salta de la rama superconductora de voltaje cero a la óhmica de $v=i$. Al aumentar la temperatura esta situación cambia. Para $T=0.02$ (gráficas situadas en la fila intermedia de la figura) se observa por un lado un aumento importante de la corriente de desanclaje al aumentar el tamaño del sistema y,

para sistemas suficientemente grandes, la aparición de la rama de difusión de bajo voltaje. Hay que mencionar no obstante que el importante cambio de la corriente de depinning con $N$ en realidad es aparente (ver figura 4.26 más adelante). A esta temperatura nos encontramos justo antes la aparición de la rama de difusión, de hecho ésta ya aparece para $N=120$ y la distribución 

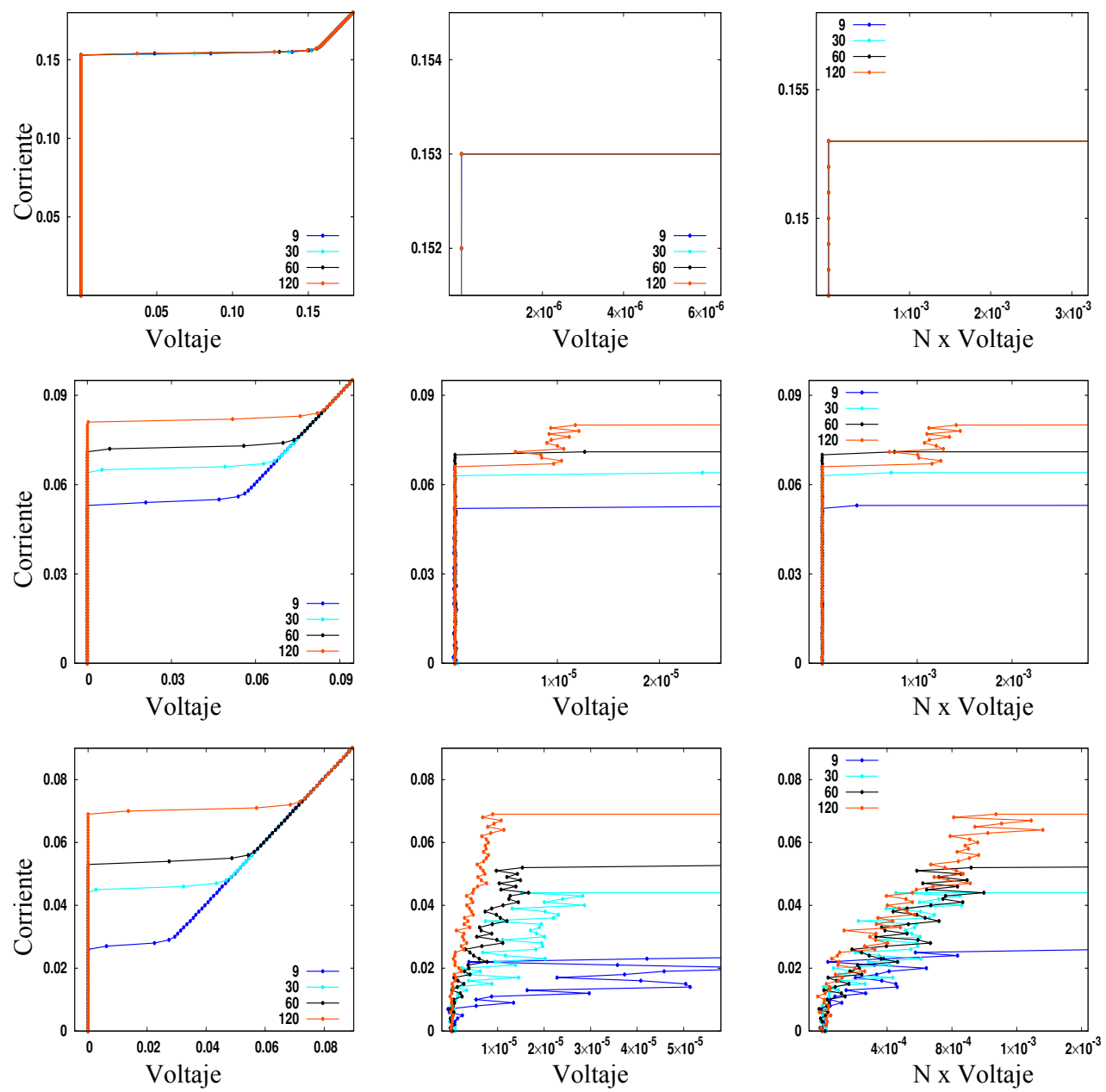

Figura 4.23: Comparación de las curvas $i$ - $v$ para un fluxón en anillos de 9,30,60,120 uniones Josephson con acoplamiento $\lambda=0.4$, y amortiguamiento de $\Gamma=0.001$ a tres temperaturas distintas: $T=0$ (fila superior), $T=0.02$ (fila central) y $T=0.1$ (fila inferior). En cada caso se representan tres figuras: curva $i$ - $v$ completa (columna izquierda), zona de bajo voltaje de la curva (columna central) y $v_{\text {fluxn }}=N v$ en la zona de bajo voltaje de la curva (columna derecha).

de probabilidad para la corriente $i_{d e p}$ es muy ancha pero el valor medio de la misma no cambia de manera importante de unos tamaños a otros de la red. Si aumentamos la temperatura a $T=0.1$, gráficas situadas en la fila inferior de la figura, se observa claramente para todos los tamaños de la red la emergencia de la rama de difusión. En este caso el salto a la rama óhmica no se corresponde con un depinning, ya que el fluxón comienza a moverse a corriente prácticamente nula, sino con el abandono de la rama de difusión. Dicho abandono ocurre a un valor de la corriente $i_{s w}$ que depende de manera notable 

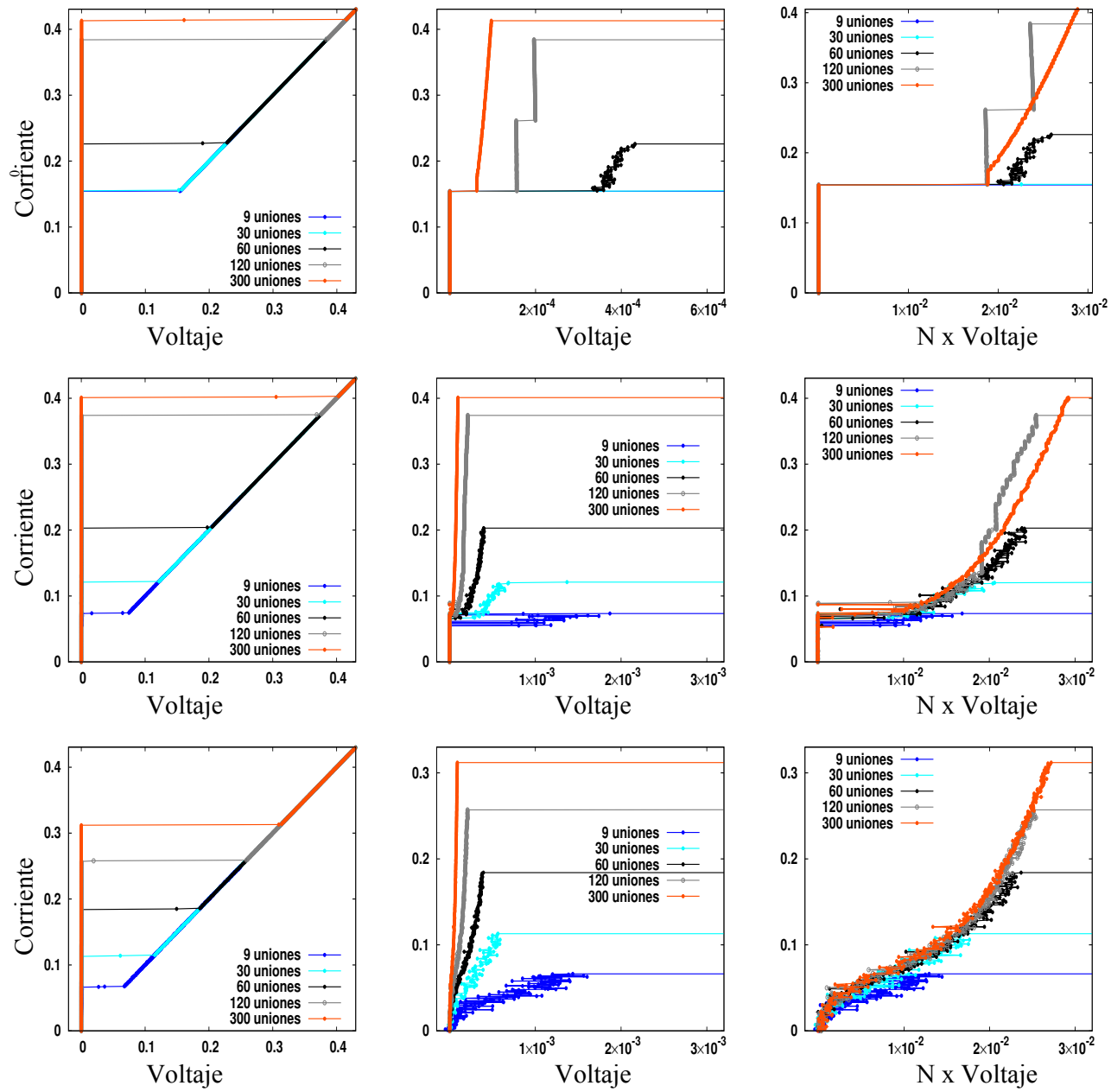

Figura 4.24: Comparación de las curvas $i$-v para un fluxón en anillos de 9,30,60,120 uniones Josephson con acoplamiento $\lambda=0.4$, y amortiguamiento de $\Gamma=0.01$ a tres temperaturas distintas: $T=0$ (fila superior), $T=0.02$ (fila central) y $T=0.1$ (fila inferior). En cada caso se representan tres figuras: curva $i$ - $v$ completa (columna izquierda), zona de bajo voltaje de la curva (columna central) y $v_{f l u x n}=N v$ en la zona de bajo voltaje de la curva (columna derecha).

del tamaño de la red. Las figuras de la columna derecha permite comparar de modo más eficiente los resultados obtenidos para distintos tamaños de red.

En la figura 4.24 se ve la dinámica del fluxón para sistemas de 9, 30, 60, 120 e incluso 300 uniones. Para $T=0$, en todos los tamaños del sistema la corriente de escape es igual a 0.154 como se aprecia en la parte central y derecha (comparación normalizada) de la figura. En el caso de temperatura $\mathrm{T}=0.02$ de la figura 4.24, el sistema presenta difusión a partir de un valor aproximado de 0.071 de la corriente $\left(i_{d e p}\right)$ para todos los tamaños del sistema, como se 

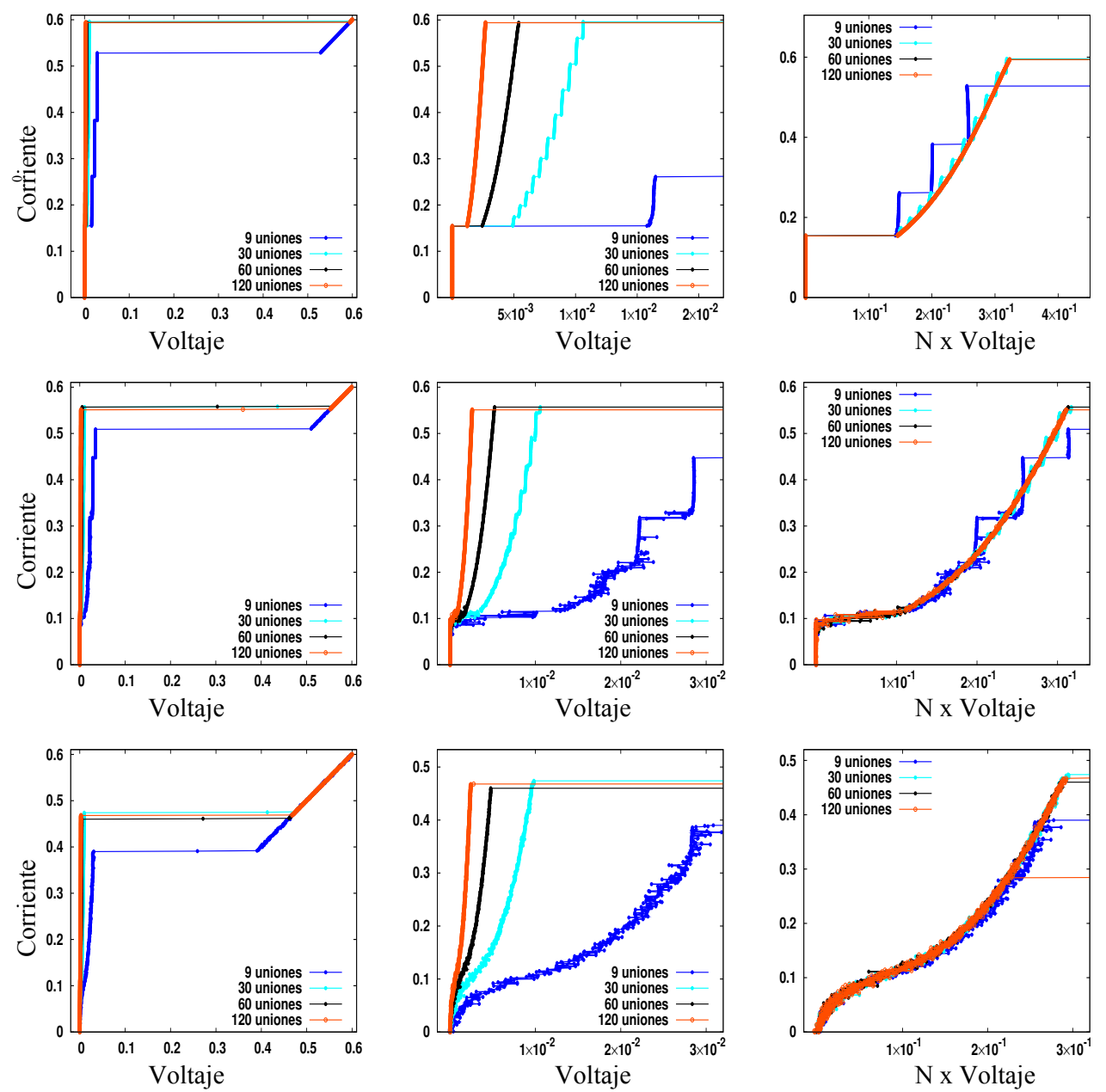

Figura 4.25: Comparación de las curvas $i-v$ para un fluxón en anillos de 9,30,60,120 uniones Josephson con acoplamiento $\lambda=0.4$, y amortiguamiento de $\Gamma=0.1$ a tres temperaturas distintas: $T=0$ (fila superior), $T=0.02$ (fila central) y $T=0.1$ (fila inferior). En cada caso se representan tres figuras: curva $i$ - $v$ completa (columna izquierda), zona de bajo voltaje de la curva (columna central) y $v_{\text {fluxn }}=N v$ en la zona de bajo voltaje de la curva (columna derecha).

aprecia en la parte central de la figura. Sin embargo, la corriente de switching se incrementa de modo importante en la medida en que el sistema es mayor. Cuando aumentamos la temperatura al sistema (figura inferior), el fluxón entre en la rama de difusión desde $i=0$, independiente del tamaño del sistema. La de switching sigue siendo notablemente diferente para los diferentes valores de $N$. En todos los casos se observa que la gráfica de $v_{\text {fluxon }}$ permite una mejor comparación de la dinámica del fluxón para los diferentes valores de $N$.

Presentaremos ahora los resultados para un amortiguamiento un orden de 
Cuadro 4.2: Temperatura crítica para la emergencia de la rama de bajo voltaje en la curva $I-V$ del fluxón para $\lambda=0.4$ y dos valores de $\Gamma$.

\begin{tabular}{|c|cccc|}
\hline & \multicolumn{4}{|c|}{$\Gamma=0.001$} \\
\hline uniones & 9 & 30 & 60 & 120 \\
\hline$T_{C}$ & 0.025 & 0.018 & 0.014 & 0.011 \\
\hline \hline & \multicolumn{4}{|c}{$\Gamma=0.01$} \\
\hline uniones & 9 & 30 & 60 & 120 \\
\hline$T_{C}$ & 0.0126 & 0.008 & 0 & 0 \\
\hline
\end{tabular}

magnitud mayor, $\Gamma=0,1$. Al aumentar este parámetro entramos en la región delas curvas $i$ - $v$ donde son apreciables los distintos peldaños de resonancias entre la velocidad de traslación del fluxón y los modos lineales de la red. En la fila superior de la figura 4.25 se muestran los resultados para $T=0$. El número de escalones de resonancia permitidos aumenta con el tamaño de la red, así para valores altos de $N$ parece observarse una curva continua para el voltaje. En todos los casos el sistema entra en la zona de resonancia a través de un depinning a $i=0.154$. En la mayoría de los casos $(N \geq 30)$ el switching a la rama óhmica se produce aproximadamente a la misma corriente, $i_{s w} \simeq 0.55$. Si aumentamos la temperatura el ruido térmico comienza a afectar los diferentes punto de saltos entre escalones (fila central) y a temperaturas suficientemente altas (fila inferior) es capaz de apantallar totalmente los saltos dando una curva ruidosa pero de apariencia continua.

\subsubsection{Estudio de $\left\langle i_{\mathrm{sw}}(T)\right\rangle$ para sistemas de diferentes tamaños}

En la sección anterior se ha podido observar que el valor de la corriente necesaria para el escape del fluxón $\left\langle i_{\text {dep }}(T)\right\rangle$ no depende del tamaño del sistema representado en el número de uniones Josephson en el anillo. Por el contrario, cuando analizamos las corrientes de switching se aprecian diferencias significativas con el cambio de tamaño del sistema. Por ello es interesante realizar un estudio de como se comporta el valor de $\left\langle i_{s w}(T)\right\rangle$ para diferentes tamaños del sistema.

En este apartado presentamos los resultados de la comparación del valor medio de la corriente de switching $\left\langle i_{\mathrm{sw}}(T)\right\rangle$ y su desviación estándar $\sigma$ en los sistemas de diferente tamaño. Los resultados fueron obtenidos desde la simulación numérica de las ecuaciones dinámicas del sistema para 200 muestras. La figura 4.26 muestra resultados para sistemas de 9, 30, 60 y 120 uniones para amortiguamiento $\Gamma=0.001$ y acoplamiento $\lambda=0.4$. La rampa promedio 

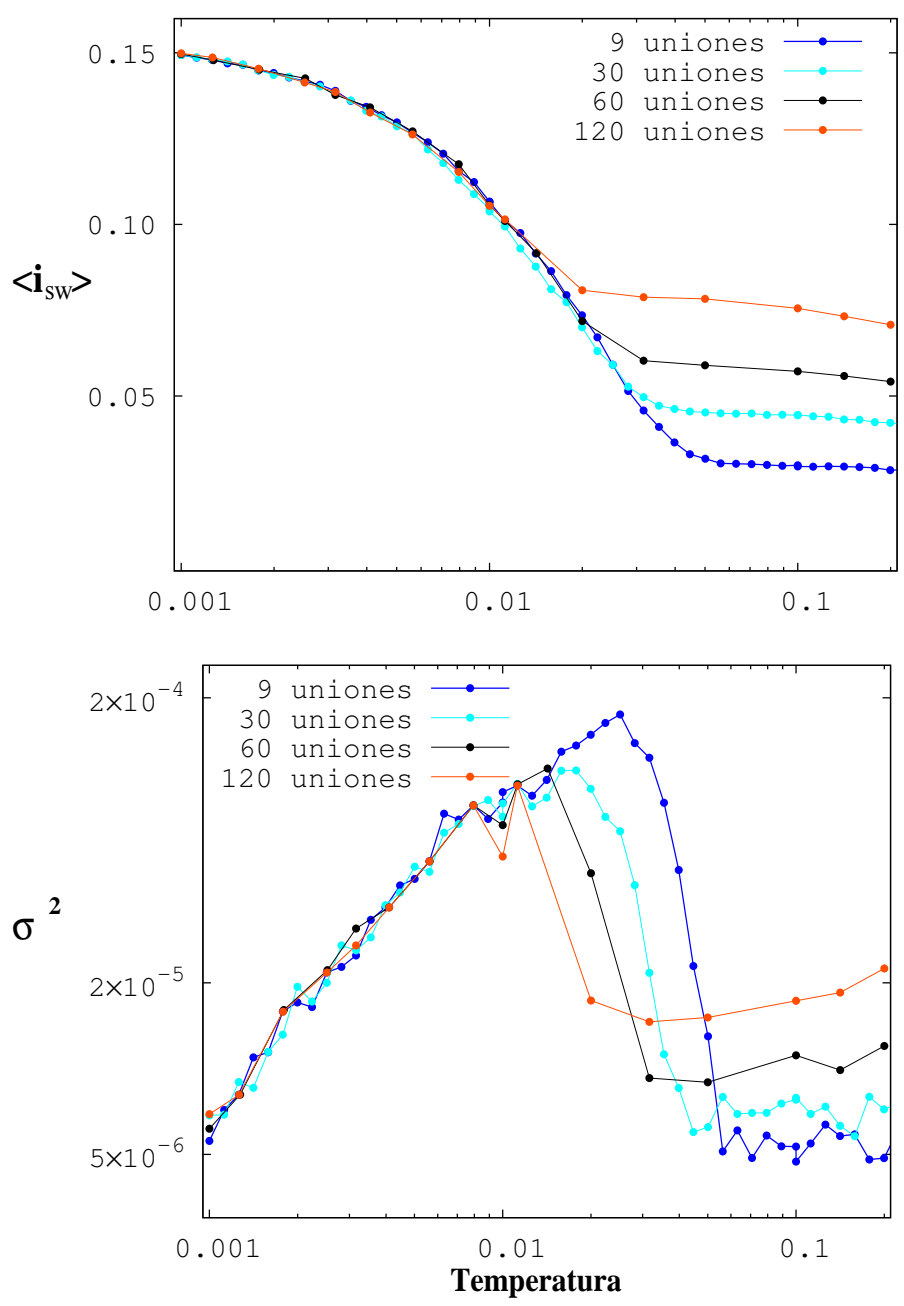

Figura 4.26: Valor medio, $\left\langle i_{\mathrm{sw}}(T)\right\rangle$, y desviación estándar, $\sigma$, de la distribución de probabilidad de la corriente de switching como función de la temperatura. El acoplamiento es $\lambda=0.4$ y el amortiguamiento de $\Gamma=0.001$.

utilizada fue de $4 / 3 \times 10^{-6}$. El comportamiento de la corriente de switching con la temperatura es similar al estudiado para el sistema de 9 uniones: la corriente de switching coincide con la corriente de depinning para valores pequeños de temperatura pero a una temperatura determinada la aparición de la rama de difusión del fluxón provoca que estas dos corrientes se diferencien y la corriente de switching se estabilice en un valor practicamente constante que depende fuertemente del tamaño de la red aumentando con este. Con respecto a la desviación estándar, se observa que a bajas temperaturas esta sigue la conocida ley de $T^{2 / 3}$ y a altas temperaturas alcanza un máximo que se puede identificar con los puntos de inflexión de la corriente $\left\langle i_{\mathrm{sw}}(T)\right\rangle$ o la temperatura 

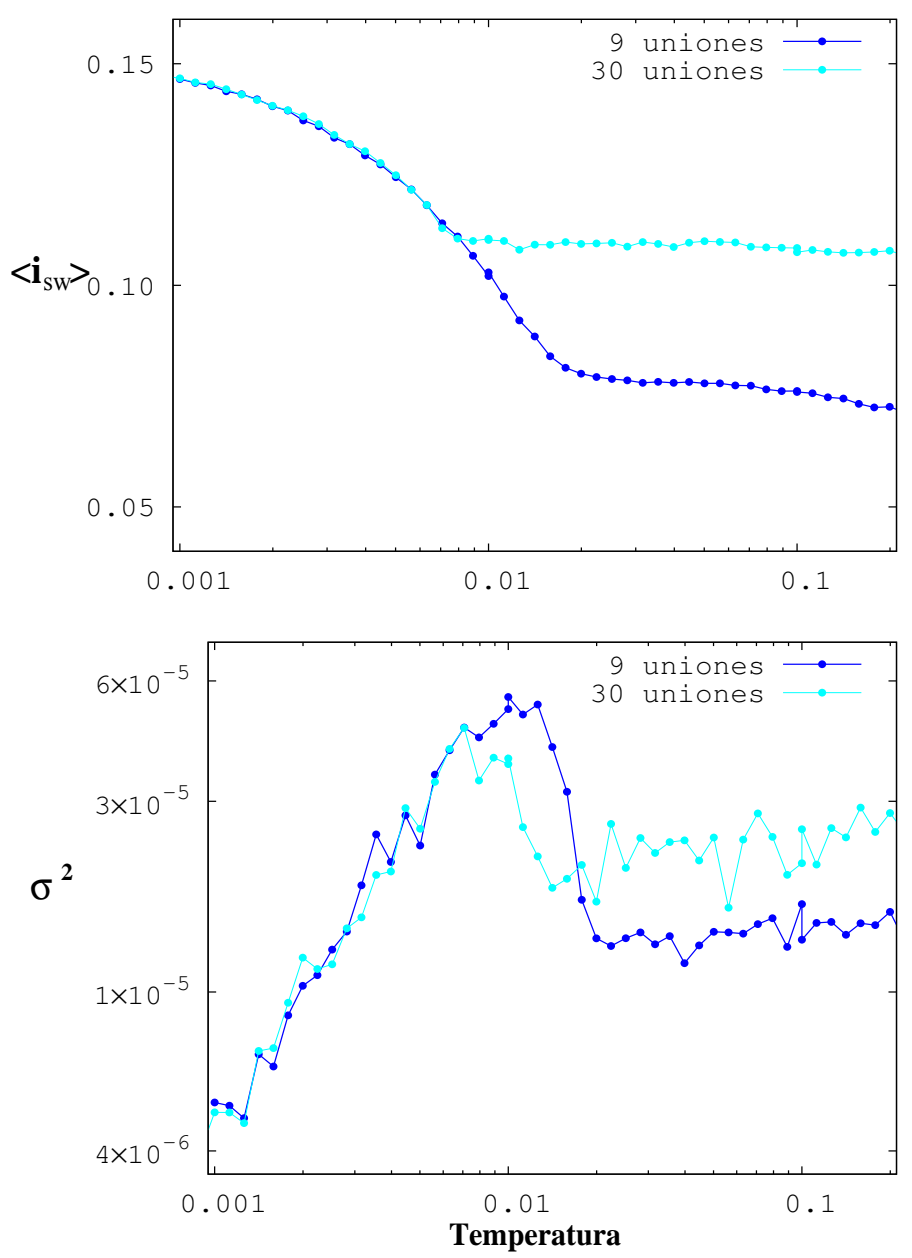

Figura 4.27: Valor medio, $\left\langle i_{\mathrm{sw}}(T)\right\rangle$, y desviación estándar, $\sigma$, de la distribución de probabilidad de la corriente de switching como función de la temperatura. El acoplamiento es $\lambda=0.4$ y el amortiguamiento de $\Gamma=0.01$.

crítica de aparición de la rama de difusión. Aumentando aún mas $T$, se observa que $\sigma$ primero decrece y luego se estabiliza en valores relativamente pequeños que aumentan con el tamaño del sistema. Podemos decir, en general, que los sistemas presentan un valor crítico de temperatura a partir del cual el valor de la corriente para el cual el arreglo de $\mathrm{N}$ uniones, salta a la rama whirling es independiente de la temperatura (zona plana de la figura 4.26). El valor aproximado de la temperaturas críticas se consigna en la cuadro 4.2 y fueron obtenidos de los picos de las curvas de desviación estandar.

La simulación de la dinámica del fluxón a un valor mayor del amortiguamiento, $\Gamma=0.01$, nos muestra un escenario en gran medida similar al expuesto 

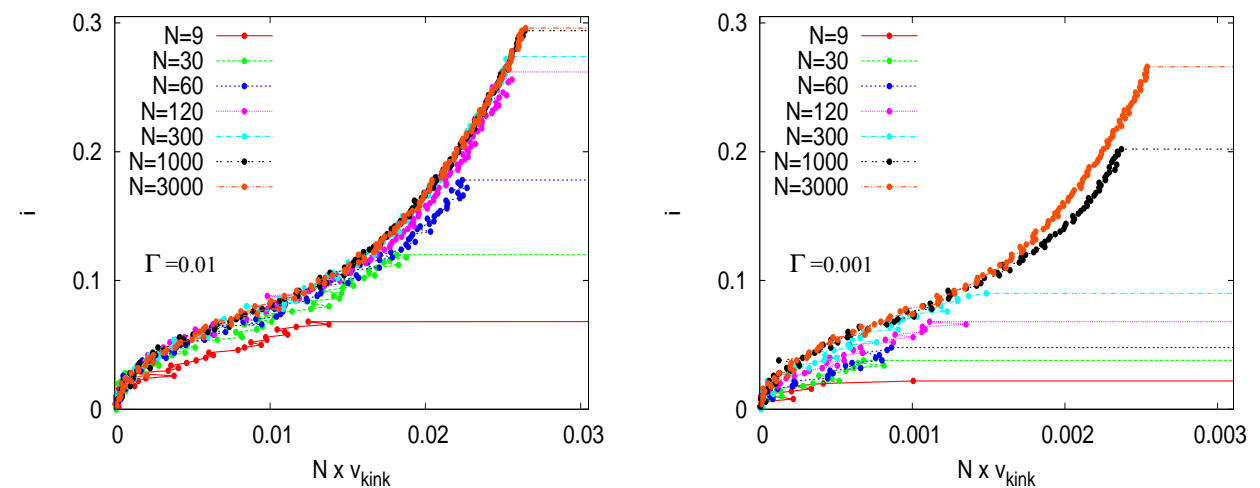

Figura 4.28: Ejemplo de curvas $i$ - $v$ para 1 fluxón, $\lambda=0.4$, varias uniones, $\Gamma=0.01$ (izquierda) y $\Gamma=0.001$ (derecha) con el voltaje normalizado al número de uniones en el sistema.

en el párrafo anterior. La figura 4.27 muestra el resultado para $\left\langle i_{s w}\right\rangle$ y $\sigma$ a este valor del amortiguamiento para redes con 9 y 30 uniones. Como puede apreciarse en la figura 4.24 a valores mayores de $N$ una rama de bajo voltaje existe desde $T=0$ y el salto a la rama óhmica se produce a valores relativamente altos de la corriente, en cualquier caso superiores a $i_{d e p}^{0}$. Entonces la distribución de la probabilidad para el salto no cambia apreciablemente al variar $T$; $\left\langle i_{s w}\right\rangle$ y $\sigma$ son prácticamente constantes en todo el intervalo de temperaturas estudiado.

Al observar la tendencia que sigue la corriente de switching con el incrememnto del número de uniones en el anillo, decidimos realizar la simulación numérica de la dinámica del fluxón con tamaños de red mayores de los estudiados en el caso de $\lambda=0.4$ y $\Gamma=0.01$ y $\Gamma=0.001$, esto se puede ver en la figura 4.28 en donde se muestra en detalle a baja voltaje y normalizado al número de uniones en el sistema, que la corriente de switching tiende a un valor límite en la medida que aumenta el número de uniones en el sistema.

\subsection{Red regular de 9 uniones con 2 y 3 fluxones}

Hasta ahora hemos presentado resultados para un fluxón en una red de uniones Josephson. No obstante también es interesante conocer el comportamiento del sistema con un número mayor de fluxones. En este caso efectos de interacción entre fluxones o de conmensurabilidad comienzan a ser relevantes. En la figura 4.1 se puede ver la distribución de las fases para configuraciones con uno, dos y tres fluxones en una red de 9 uniones. En las próximas páginas 

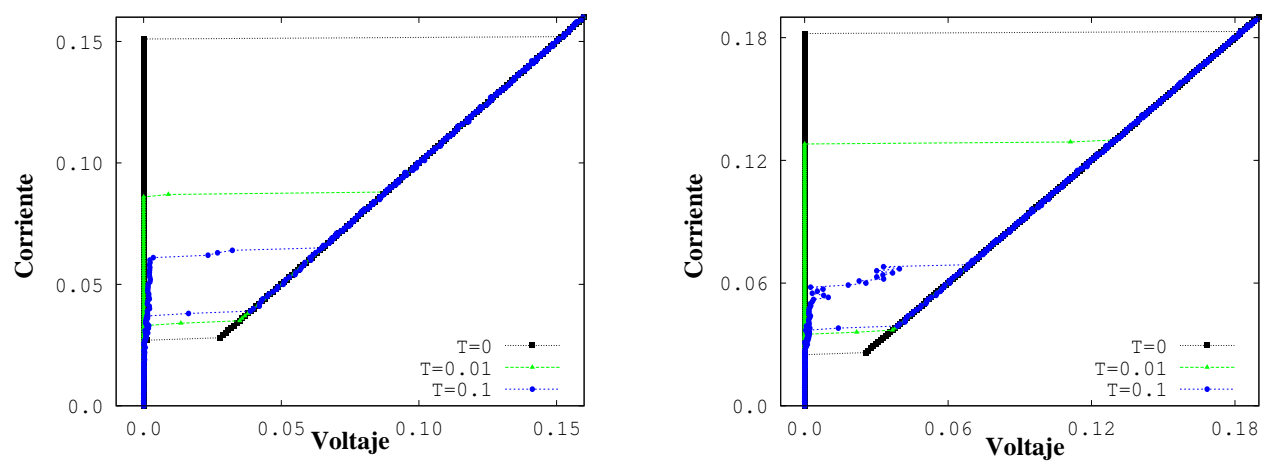

Figura 4.29: Ejemplo de curvas $i-v$ para 2 fluxones (izquierda) y 3 fluxones (derecha) en un anillo de 9 uniones Josephson con $\lambda=0.4$, amortiguamiento $\Gamma=0.01$ y 3 valores de temperatura ( $T=0,0.01$ y 0.1 ). Mostramos tanto la curva aumentando la corriente como disminuyendo la misma, pudiéndose apreciar una amplia zona de biestabilidad.

estudiaremos algunos aspectos de la dinámica de redes de 9 uniones con dos o tres fluxones atrapados. Los aspecto fundamentales de la dinámica en sistemas de anillos de uniones con dos o tres fluxones son similares a los descritos en el caso de un único fluxón. Por ejemplo, en la figura 4.29 se puede ver la histéresis y regiones de difusión a temperaturas altas. Pretendiendo continuar con el sistema discreto y subamortiguado, en la figura 4.29 se ha simulado un sistema de 9 uniones Josephson con $\lambda=0.4$, amortiguamiento $\Gamma=0.01$, para 2 fluxones (izquierda) y 3 fluxones (derecha), mostrándose las curvas $I$ - $V$ tanto al aumentar la corriente desde cero como al disminuirla desde un estado de alto voltaje.

A continuación mostraremos primero el estudio de las curvas $I$ - $V$ de una red de 9 uniones con dos fluxones. Más tarde realizaremos el mismo análisis para una red con 3 fluxones. Centraremos nuestro estudio en dos valores del parámetro de acoplamiento, $\lambda=0.4$ y 0.8 y, como ha sido habitual hasta ahora, analizaremos diferentes valores del amortiguamientos y de la temperatura. Finalmente establecemos una comparación directa de resultados con 1, 2 o 3 fluxones atrapados tanto a nivel del estudio de las curvas $I-V$ como de la distribución de las corriente de desanclaje y de switching según el caso.

\subsubsection{2 fluxones}

En la figura 4.30 podemos observar la dinámica del arreglo en presencia de ruido térmico, en este caso de acoplamiento $\lambda=0.4$ y tres valores del amortiguamiento: $\Gamma=0.01, \Gamma=0.1$ y $\Gamma=1$. Mostramos simulaciones para cinco valores de la temperatura: $T=0, T=0.002, T=0.01, T=0.02$ y $T=0.1$. Esta 

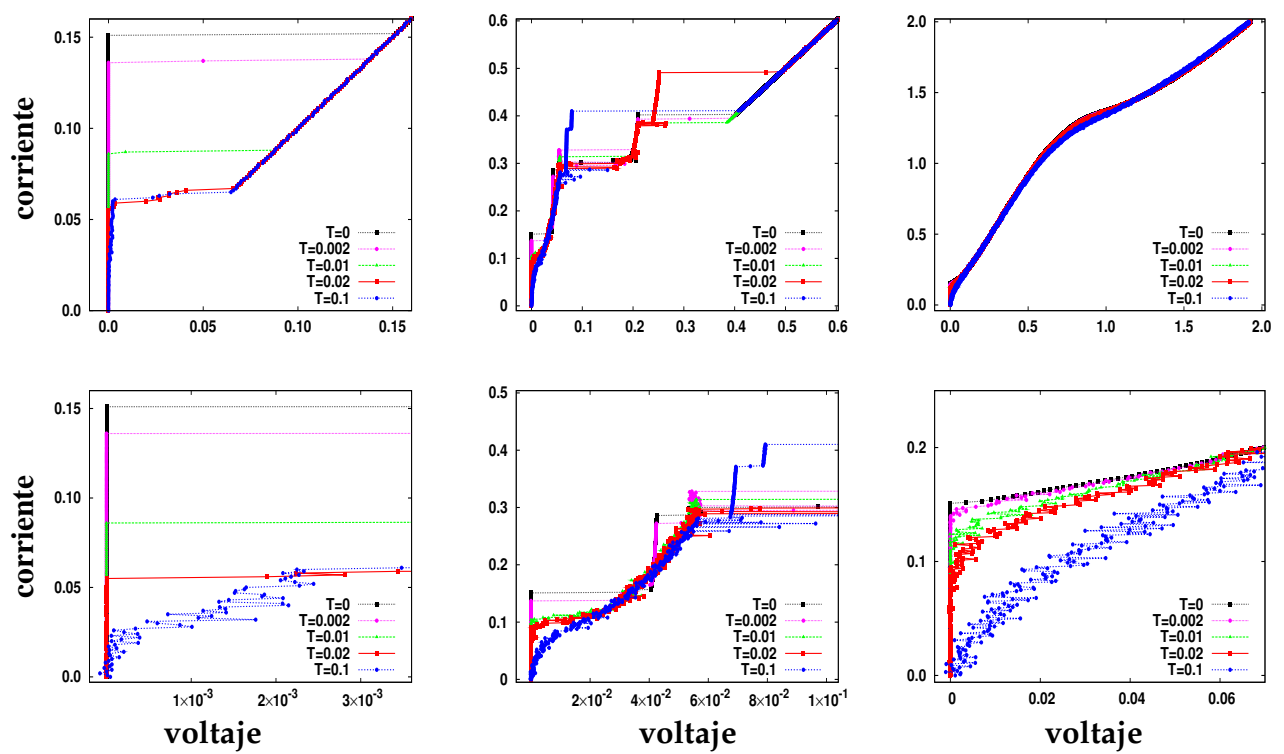

Figura 4.30: Curvas $I$ - $V$ para 2 fluxones en un anillo de 9 uniones Josephson con $\lambda=0.4$ a $\Gamma=0.01$ (izquierda), $\Gamma=0.1$ (medio) y $\Gamma=1.0$ (derecha). Cada curva muestra 5 temperaturas diferentes $(T=0,0.002,0.001,0.02,0.1)$.

figura debe compararse con la figura 4.7 donde se muestra la misma simulación para el caso de un único fluxón.

Para este valor del acoplamiento $\lambda$ la corriente de desanclaje a temperatura cero del sistema con dos fluxones es $i_{\text {dep }}^{0} \approx 0.151$ (un poco menor que la encontrada para un fluxón, $i_{\mathrm{dep}}^{0} \approx 0.155$ ). Tal y como se observa para el caso de un fluxón, notamos que el valor de la corriente de desanclaje va disminuyendo a medida que aumenta el valor de la temperatura. A temperaturas bajas ese desanclaje supone el salto a la rama de alto voltaje pero a temperaturas suficientemente altas el desanclaje supone un salto a la rama de bajo voltaje de difusión para el fluxón que a corrientes mayores sufre el salto a la rama óhmica.

Las gráficas centrales muestran el resultado para un valor superior del amortiguamiento, ahora $\Gamma=0.1$. Este valor corresponde con la zona de resonancias de la curva $i$ - $v$ del sistema. Quizás la diferencia más notable respecto a los resultados para un fluxón lo encontramos al disminuir la corriente $i$, ahora la curva vuelve a través de las distintas resonancias mientras que para un fluxón se observaba un reatrapamiento directo desde la zona de alto voltaje. Por otro lado a diferencia con el sistema de un fluxón, la dinámica a temperatura alta presenta una histéresis más notable como también se aprecia en la figura 4.29, en donde se ha dibujado el proceso completo, desde el desanclaje con el in- 

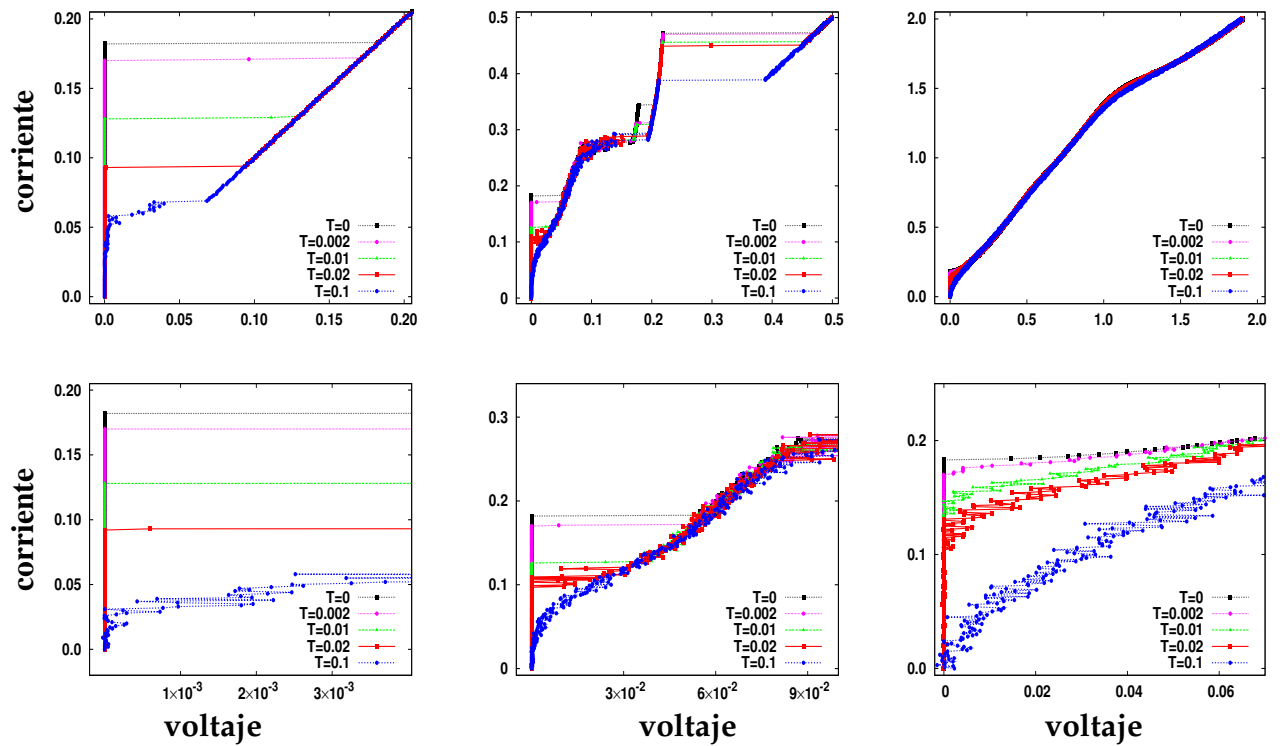

Figura 4.31: Curvas $I$ - $V$ para 3 fluxones en un anillo de 9 uniones Josephson con $\lambda=0.4$ a $\Gamma=0.01$ (izquierda), $\Gamma=0.1$ (medio) y $\Gamma=1.0$ (derecha). Cada curva muestra 5 temperaturas diferentes $(T=0,0.002,0.001,0.02,0.1)$.

cremento de corriente para $\lambda=0.4$ y $\Gamma=0.01$, a temperatura cero, 0.01 y 0.1 , hasta cuando el sistema se ancla nuevamente con el decrecimiento de la corriente. En la imagen ampliada, se puede apreciar mejor que en el caso de dos fluxones, los steps observados son menores en número que en el sistema con un fluxón, pero a la vez, son más prolongados. Además los voltajes obtenidos son mayores ya que en este caso se tiene la contribución de los dos fluxones moviéndose en resonancia a lo largo de la red. Sin embargo a la temperatura más alta, esto es, $T=0.1$, se aprecian unos pasos adicionales previos al salto de la rama óhmica, con una gran fluctuación antes del primer step.

Finalmente, para valores mayores del amortiguamiento alto, $\Gamma=1.0$, se observa un redondeo del dibujo de la dinámica, tal como en el caso de 1 fluxón. En este caso la zona de menor voltaje da cuenta del movimiento de difusión de dos fluxones y por lo tanto el voltaje asociado a la misma será doble del observado para el caso de un fluxón.

\subsubsection{3 fluxones}

Pasamos ahora a analizar el caso de sistemas con 3 fluxones en el anillo. Los resultados se presentan en la 4.31, que en este caso debe compararse con las figuras 4.7 y 4.30. La corriente de desanclaje a temperatura cero resulta ser 

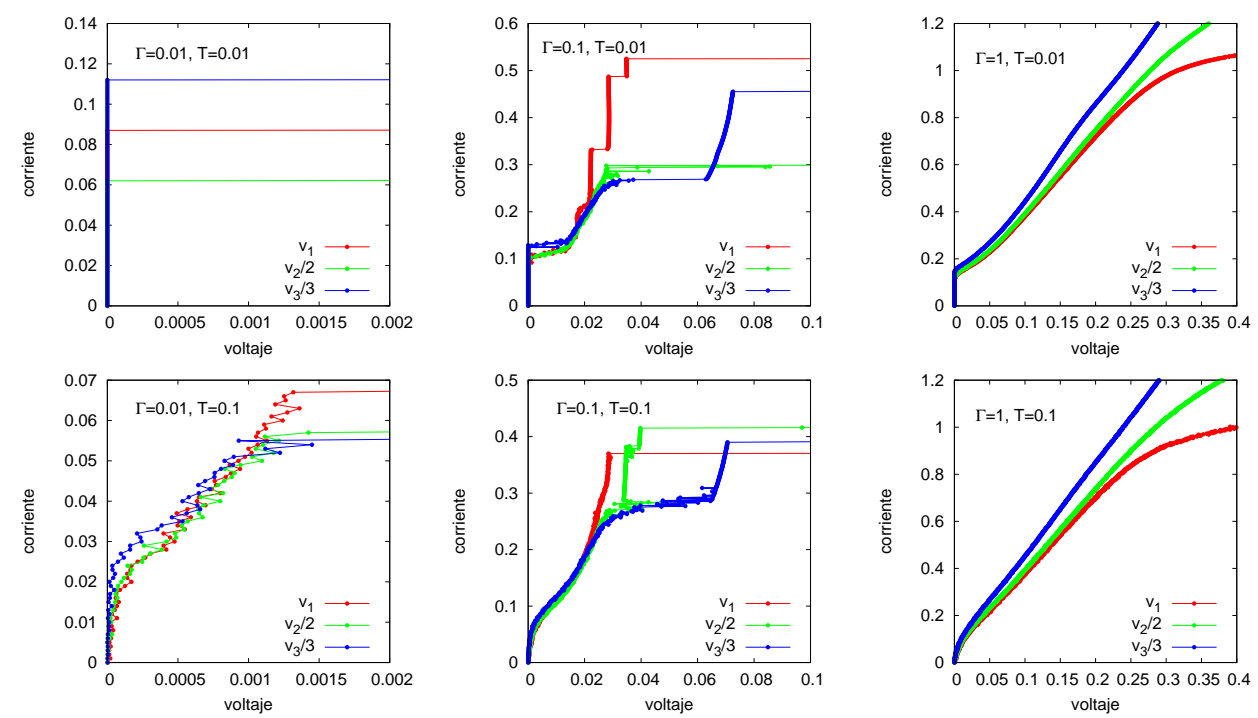

Figura 4.32: Comparación de las zonas de bajo voltaje de las curvas $i-v$ para sistemas con 1,2 y 3 fluxones en un anillo de 9 uniones Josephson con $\lambda=0.4$, a $T=0.01$ (figuras superiores) y $T=0.1$ (figuras inferiores) y $\Gamma=0.01$ (izquierda), $\Gamma=0.1$ (centro) y $\Gamma=1.0$ (derecha). En cada caso se muestra $v_{j}=v \times \mathrm{n}^{0}$ de fluxones con $j=\mathrm{n}^{0}$ de fluxones.

$i_{\text {dep }}^{0} \approx 0.183$, sensiblemente mayor que en el caso de uno y dos fluxones. Dicho aumento manifiesta un efecto de conmmensurabilidad en el sistema: tenemos un sistema con tres fluxones y nueve uniones lo que resulta en una densidad $1 / 3$ de fluxones en la red. De nuevo para valores bajos del amortiguamiento se observa el importante decrecimiento de la corriente de desanclaje con la temperatura y la aparición a altas temperaturas de una rama de difusión de bajo voltaje. La figura central muestra la existencia de numerosas resonancias, de mayor estabilidad y valor que las encontradas en los ejemplos anteriores y afectadas de manera importante por la temperatura aunque las resonancias de mayor voltaje resultan ser robustas incluso a los valores mayores de $T$. Para valores del amortiguamiento alto, es decir, $\Gamma=1.0$, se observa un redondeo en la curva dinámica, tal como en los casos de uno y dos fluxones. De nuevo los voltajes son mayores ya que en este caso debe considerarse la contribución al voltaje total de la difusión de tres fluxones.

\subsubsection{Comparación entre los sistemas de 9 uniones Josephson con diferente número de fluxones}

En los sistemas de uniones Josephson, con más de un fluxón, la dinámica del sistema presenta algunas diferencias que se hacen más notables al realizar 
una comparación directa de las curvas $I-V$ obtenidas. Estamos interesados en la región de bajo voltaje, donde el voltaje del sistema está dado por la contribución de cada fluxón al total, por ello en este caso mostraremos valores de $v / M \operatorname{con} M$ el número de fluxones en el sistema.

La figura 4.32 muestra los resultados obtenidos para un conjunto de valores de los parámetros de interés. Los resultados a $T=0.01$ se muestran en las gráficas superiores. Para bajo amortiguamiento, $\Gamma=0.01$ (izquierda), no se observa rama de bajo voltaje y en todo caso el sistema salta desde voltaje cero a la rama de alto voltaje. Aumentando el amortiguamiento del sistema a $\Gamma=0.1$ se observa que la dinámica no es una mera suma de las contribuciones de fluxones independientes sino que hay efectos de interacción entre los mismos. Para mayor amortiguamiento $\Gamma=1$ la curva no presenta discontinuidades como es de esperar y de nuevo se observan diferencias entre los tres casos que son pequeñas en la comparación de $M=1$ y $M=2$ y algo mayores para $M=3$.

En la fila inferior de la figura 4.32 se muestra el caso $T=0.1$. En este caso a bajo $\Gamma$ (izquierda) la temperatura $T=0.1$ es suficientemente alta para excitar la rama de difusión del fluxón. Vemos como la contribución al voltaje de cada uno de los fluxones es similar y las corrientes de salto a la rama óhmica también, como confirmaremos en la siguiente sección. Las curvas de amortiguamiento mayor son más parecidas a las de $T=0.01$. A $\Gamma=0.1$ se observan ramas de difusión y resonancia combinadas con diferencias importantes en la parte de las resonancias según el valor de $M$. Para $\Gamma=1$. la dinámica es sobreamortiguada, suaviza por el efecto térmico y con un apreciable efecto de conmensurabilidad para $M=3$.

\subsubsection{Resultados sobre $\left\langle i_{\mathrm{dep}}(T)\right\rangle,\left\langle i_{\mathrm{sw}}(T)\right\rangle, \mathbf{y} \sigma$}

A continuación vamos a pasar a mostrar las curvas para el valor medio de la corriente de depinning y la corriente de switching, y sus respectivas desviaciones medias, para los casos de uno, dos y tres fluxones en una red con 9 uniones y a distintas temperaturas y $\operatorname{con} \lambda=0.4$ y $\Gamma=0.01$

La figura 4.33 muestra el análisis de la corriente de desanclaje media $\left\langle i_{d e p}\right\rangle$. Los resultados para uno y dos fluxones son muy cercanos a baja temperatura, esto es debido a que la corriente de depinning a temperatura nula de los dos casos es muy similar. La curva del caso de tres fluxones se aleja de las anteriores en consonancia con que este sistema muestra una corriente determinista de desanclaje mayor.

Con respecto a la desviación estándar, podemos ver que esta crece con $T^{2 / 3}$ en todos los sistemas de 1,2 y 3 fluxones, como lo predice la teoría de activación térmica estándar y alcanza un máximo a altas temperaturas, cuando 

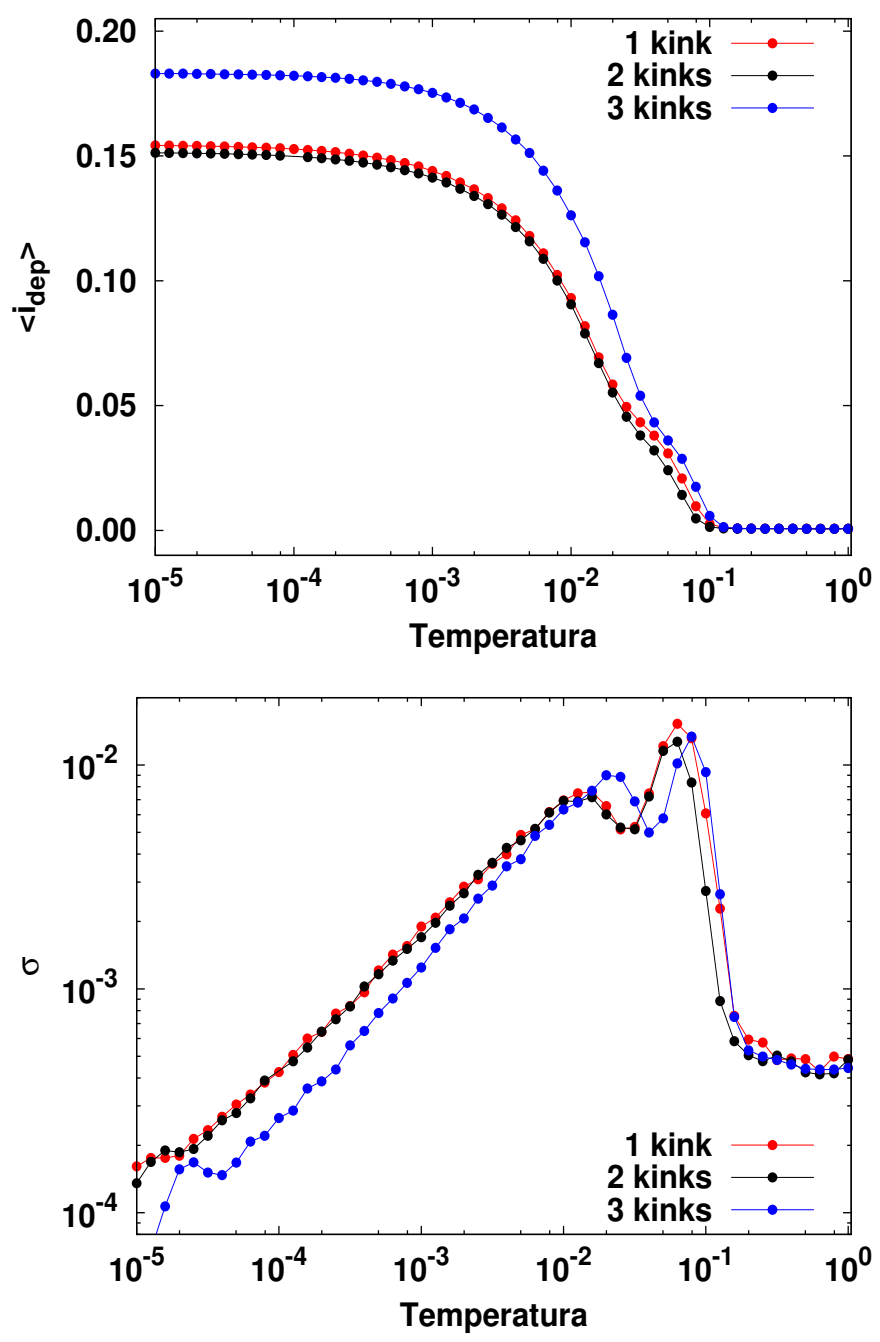

Figura 4.33: $\left\langle i_{\text {dep }}\right\rangle$ y $\sigma$ versus $T$ a $\Gamma=0.01$ y $\lambda=0.4$ para sistemas con 1,2 y 3 fluxones.

$\left\langle i_{\text {dep }}\right\rangle \rightarrow 0$. Entonces la desviación estándar decrece ya que todos los eventos de escape suceden en un estrecho rango de valores de corriente.

Los resultados para el caso de la corriente de switching, que señala la transición al estado de alto voltaje del sistema, son mostrados en la figura 4.34. Como ocurre en el caso de un fluxón para valores pequeños de la temperatura el salto se produce desde la rama de voltaje 0, el depinning coincide con el switching. Sin embargo existe una temperatura a partir de la cual se excita la zona de bajo voltaje del sistema y el salto a la rama óhmica se produce desde esta rama de bajo voltaje. A partir de entonces ambas corrientes críticas ya 

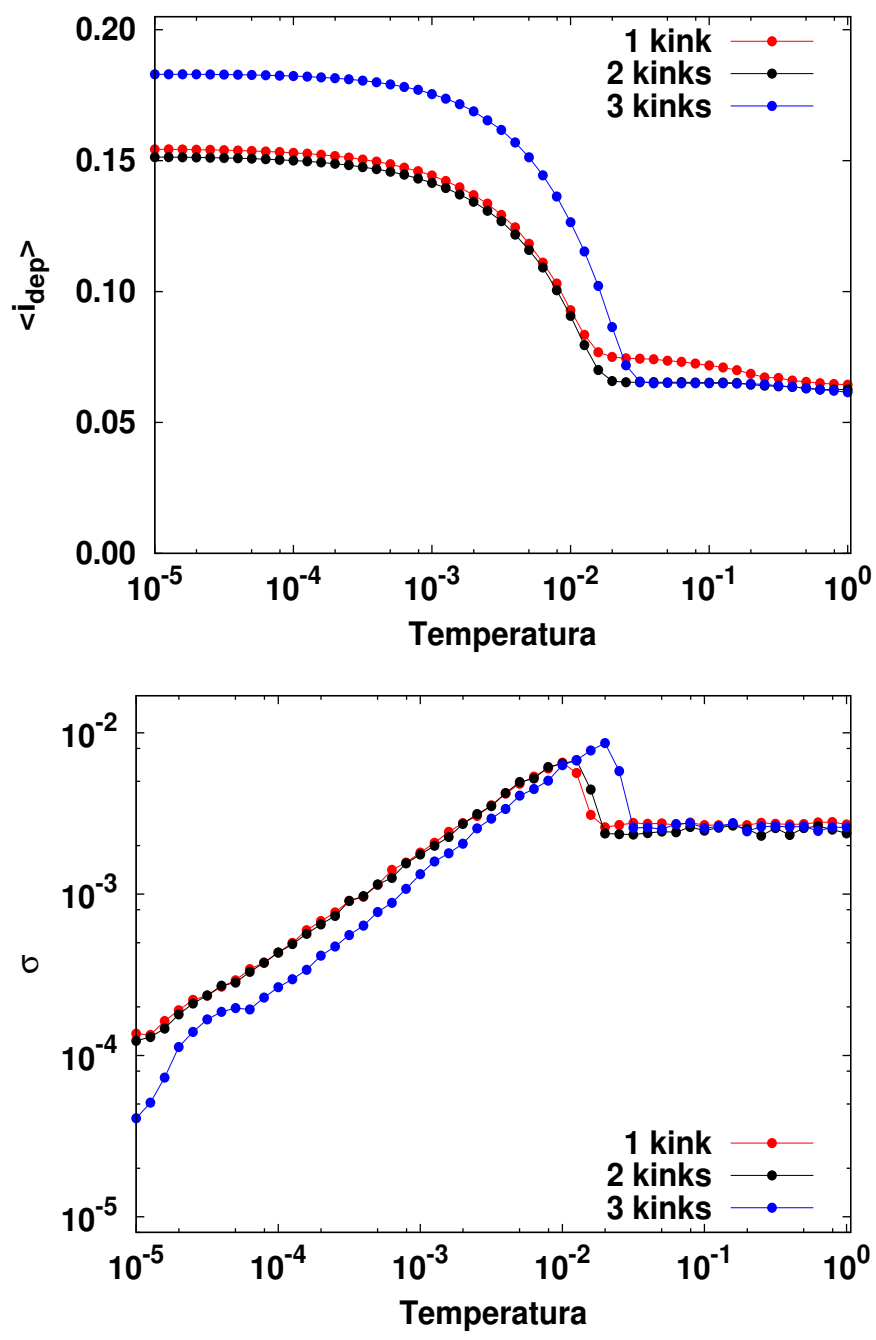

Figura 4.34: $\left\langle i_{\mathrm{sw}}\right\rangle$ y $\sigma$ versus $T$ a $\Gamma=0.01$ y $\lambda=0.4$ para sistemas con 1,2 y 3 fluxones.

no coinciden y el valor de la corriente de switching es prácticamente el mismo para los tres casos estudiados y la función densidad de distribución probabilidad asociada es bastante estrecha, como muestra el dibujo de la desviación estándar de la misma. 


\section{Capítulo 5}

\section{Desanclaje térmico de fluxones en redes ratchet}

\subsection{Introducción}

Mencionamos en el capítulo 2 que el estudio del movimiento direccional de partículas también ha cobrado relevancia en el campo de la nano y microtecnología de dispositivos superconductores, en el diseño y estudio de sistemas que permitan el movimiento direccional de vórtices y fluxones en sistemas superconductores $[85,78]$ en general y en redes de uniones Josephson en particular [83, 84], conformando lo que hemos llamado dispositivos ratchet para fluxones. La flexibilidad del sistema nos permite combinar uniones y celdas de tamaños diferentes lo que como veremos posibilita el diseño de dispositivos con potenciales substrato periódicos asimétricos [83, 84, 85].

Una manera de estudiar experimentalmente ratchets solitónicos es utilizar dispositivos basados en uniones Josephson. En estos sistemas la corriente externa es el análogo a una fuerza que actúa sobre el solitón y el voltaje medido se corresponde con la velocidad del mismo. En 1999, Falo et al [83] propusieron usar un arreglo paralelo de uniones Josephson con corrientes críticas y áreas de la unión alternantes. En este sistema los parámetros del dispositivo se puede escoger de forma que el potencial efectivo para los fluxones carezca de simetría de inversión (potencial ratchet). Como en el caso de la cadena regular, el sistema se puede modelizar por un conjunto de péndulos no-lineales acoplados, pero ahora los péndulos tienen longitudes alternantes y acoplamientos armónicos asimétricos. En su publicación, también muestran que el fluxón se comporta como una partícula en donde se puede verificar las predicciones hechas para ratchet térmico sobreamortiguado. Posteriormente Trías et al [84, 85] utilizando la configuración ratchet, diseñaron arreglos circulares y probaron algunas 


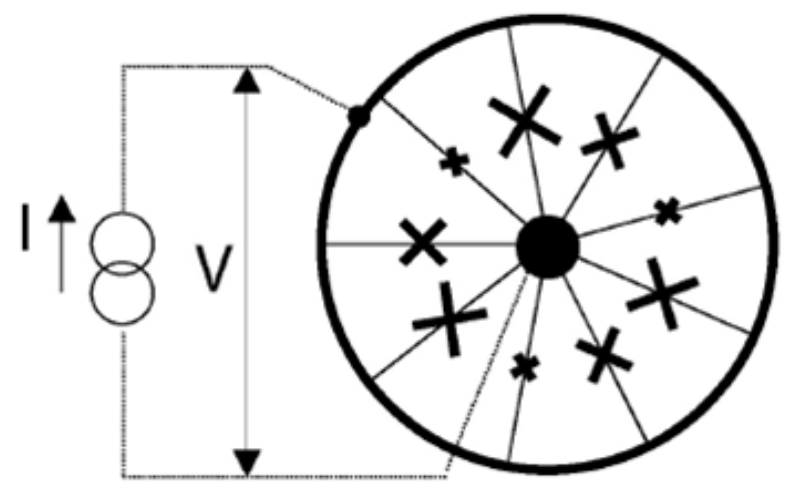

Figura 5.1: Diagrama de un arreglo tipo ratchet, las cruces representan las uniones Josephson y su variado tamaño representa diversos valores de sus parámetros que conducen a obtener una red ratchet.

de las propiedades del fluxón mostrando que las predicciones teóricas están de acuerdo con los experimentos. En particular se midieron experimentalmente el desanclaje de kinks atrapados en potenciales ratchet usando un arreglo circular de uniones Josephson. Encontraron que la corriente de desanclaje depende de la dirección de la corriente aplicada en el anillo ratchet. Además se propuso la red con uniones con tres corriente críticas como un sistema más adecuado para estudiar las propiedades de fluxones en redes ratchet de uniones Josephson.

Dada la relevancia del efecto ratchet, plasmada en el capítulo 2 y la posibilidad de estudiarlo experimentalmente, es conveniente realizar un análisis por medio de la simulación numérica de la dinámica de los fluxones en las redes tipo ratchet. En este capítulo extenderemos el estudio presentado en el capítulo anterior al caso de redes tipo ratchet formadas por celdas iguales pero uniones con tres corrientes críticas diferentes (figura 5.1) sucediéndose en relación 1:0.5:0.3. Las propiedades de equilibrio de fluxones en este tipo de redes ya fueron presentadas en el capítulo 3.

\subsection{Ecuaciones}

Como se ha visto en el capítulo 2, las principales propiedades físicas de las uniones Josephson están dadas por las relaciones Josephson:

$$
V=\frac{\Phi_{0}}{2 \pi} \dot{\varphi}, \quad I=I_{c} \sin \varphi
$$

Usando técnicas de litografía es posible construir arreglos de uniones Josephson bien caracterizadas. En este capítulo consideramos arreglos circulares 
de uniones Josephson conectadas en paralelo en configuración de anillo, alternando las corrientes críticas, como se puede observar en la figura 5.1. Como vimos en el capítulo 3, el sistema de ecuaciones que describe la dinámica de lared, en su aproximación más sencilla, puede escribirse como:

$$
h_{j}\left(\ddot{\varphi}_{j}+\Gamma \dot{\varphi}_{j}+\sin \varphi_{j}+\tilde{i}_{j}\right)=\lambda\left(\varphi_{j+1}-2 \varphi_{j}+\varphi_{j-1}\right)+i
$$

donde $j=1, \ldots N$ y $\varphi_{j}$ es la diferencia de fase invariante gauge de cada unión. La corriente esta normalizada por la corriente crítica mayor de la uniones $I_{c *}$, de modo que los $h_{j}=I_{c j} / I_{c *}$, y el tiempo por la frecuencia de plasma $\omega_{p}=$ $\sqrt{2 \pi I_{c *} / \Phi_{0} C_{*}}\left(\Phi_{0}=h / 2 e\right.$ es el cuanto de flujo magnético y $C_{*}$ es la capacitancia de la unión tomada como referencia). El parámetro $\Gamma=\sqrt{\Phi_{0} / 2 \pi I_{c *} C_{*} R_{*}^{2}}$ mide el grado de amortiguamiento en el sistema y el último término $\tilde{i}_{j}(\tau)$ describe el efecto del ruido térmico en la dinámica y satisface $\left\langle\tilde{i}_{j}(\tau)\right\rangle=0 \mathrm{y}$ $\left\langle\tilde{i}_{j}(\tau) \tilde{i}_{k}\left(\tau^{\prime}\right)\right\rangle=2 h_{j} \Gamma T \delta_{j k} \delta\left(\tau-\tau^{\prime}\right)$ donde usamos $T$ para la temperatura normalizada $T=k_{B} T_{\exp } / E_{J}$ (con $E_{J}$ la energía Josephson $E_{J}=\Phi_{0} I_{c *} / 2 \pi$ ). El acoplamiento entre las uniones está dado por el parámetro $\lambda=\Phi_{0} /\left(2 \pi L I_{c *}\right)$. El voltaje normalizado $v$ muestra la respuesta del sistema a la corriente externa, está definido por $v=V_{d c} / I_{c *} R_{*}=\left(\Phi_{0} / 2 \pi I_{c *} R_{*}\right)\langle d \varphi / d t\rangle=\Gamma\langle d \varphi / d \tau\rangle$. En las gráficas en las que se muestra la curva $I$ - $V$ del anillo dibujaremos en realidad la curva $i$ - $v$ del mismo; es decir, dibujamos corriente y voltajes normalizados. Remarcamos que las condiciones de frontera están definidas por la topología del arreglo. Aquí hemos considerado arreglos circulares de modo que $\varphi_{j+N}=\varphi_{j}+2 \pi M$ donde el número $M$ de fluxones atrapados en el sistema es una constante del movimiento.

En este capítulo consideramos el caso de 1 y 2 fluxones, para un tamaño de red de 9 uniones. En cada caso sometemos al fluxón a ambos sentidos de la corriente, que desde ahora denominaremos corriente a las corrientes negativas por $i-$ y a las positivas por $i+$. En las redes que estudiaremos $\left\{h_{j}\right\}=\{1.0,0.5$, $0.3,1.0,0.5,0.3,1.0,0.5,0.3\}$ y $\lambda>0.25$. En este caso el fluxón experimenta un potencial efectivo periódico asimétrico con un sólo mínimo por periodo de potencial (ver capítulo 3).

\subsection{Red ratchet con 9 uniones y un fluxón}

En esta sección presentamos las simulaciones numéricas de la dinámica de un fluxón en un arreglo ratchet de 9 uniones Josephson. Tal y como hicimos en el caso de las redes regulares, comenzamos mostrando las curvas $I$ - $V$ del sistema. Dada la asimetría del potencial, en este caso será necesario realizar curvas con las dos polaridades de la corriente aplicada. Más adelante se mostrará el estudio del corriente de desanclaje y la corriente de salto medias para distintos valores de la temperatura del sistema. 

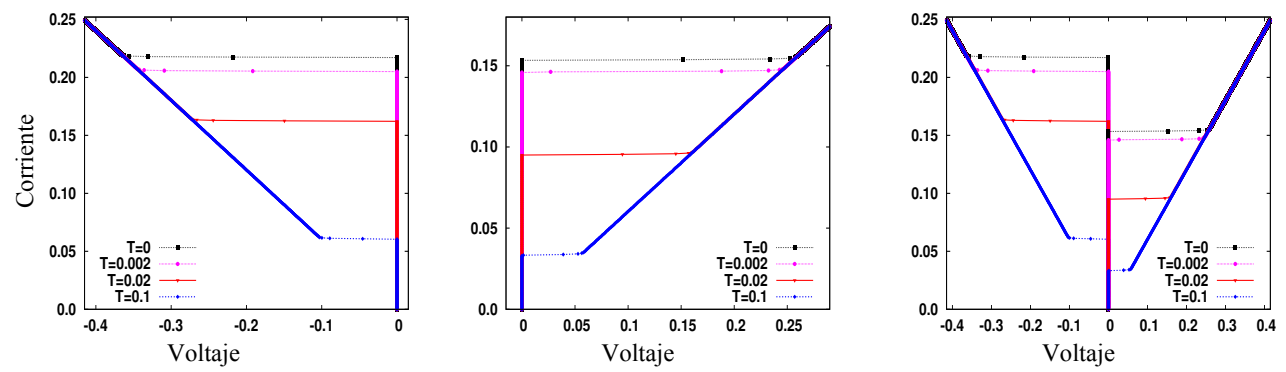

Figura 5.2: Dinámica del fluxón en un anillo ratchet de 9 uniones $\lambda=0.4$ y $\Gamma=0.001$, dos direcciones de la corriente $i-$ a la izquierda, $i+$ en el centro y la comparación de las dos direcciones de la corriente a la la derecha de la figura. Cada figura muestra 4 valores de temperatura $(T=0,0.002,0.02$ y 0.1$)$. La figura de la izquierda en realidad muestra la curva $|i|-v$.

\subsubsection{Curvas $I-V$}

Los parámetros que vamos a tener en cuenta son similares a los parámetros tenidos en cuenta en la red regular. En particular los valores del acoplamiento son adecuados para generar un potencial efectivo para el fluxón asimétrico y con un único mínimo por periodo del potencial. Además, como hemos discutimos en el capítulo 3, puede observarse como la asimetría es más acentuada para valores de $\lambda \simeq 0.4$. Este valor nos marca un óptimo en cuanto a la elección de dicho parámetro en el diseño de una red. Los valores de los acoplamientos considerados aquí son: $\lambda=0.4, \lambda=0.8$ y adicionalmente $\lambda=0.6$, el amortiguamiento estará entre $\Gamma=0.001$ y $\Gamma=1.0$ para $\lambda=0.4$ y en los demás valores del acoplamiento $\lambda=0.6$ y $\lambda=0.8$ mostraremos la dinámica para $\Gamma=0.001$, $\Gamma=0.01$ y $\Gamma=0.1$. En todos los casos para diferentes valores de la temperatura y las rampas ajustadas a los valores del acoplamiento. En el primer caso del acoplamiento $\lambda=0.4$ y para el caso particular del amortiguamiento de $\Gamma=0.001$, mostramos en la figura 5.2 la dinámica del sistema por medio de la curva $I-V$. A la izquierda de la figura 5.2, representamos la dinámica con corriente negativa $i$ - para 4 valores de la temperatura $T=0,0.002,0.02$ y 0.1 (por conveniencia se ha dibujado el valor absoluto de la corriente frente al voltaje). En el centro se representa el caso de corriente positiva. El comportamiento es similar al de la red regular, es decir, con el incremento de la temperatura, el valor de corriente necesario para el desanclaje, disminuye significativamente. En este caso $i_{d e p}^{-}(T=0)=-0.217$ y $i_{d e p}^{+}(T=0)=0.154$. En la parte derecha de la figura 5.2 se consigna el dibujo de las dos direcciones de la corriente en que se fuerza al fluxón a moverse, y utilizamos este dibujo como una forma de comparar las corrientes de desanclaje del fluxón para los diferentes valores de la temperatura. En lo que sigue del capítulo, presentaremos las curvas $I$ - $V$ de 

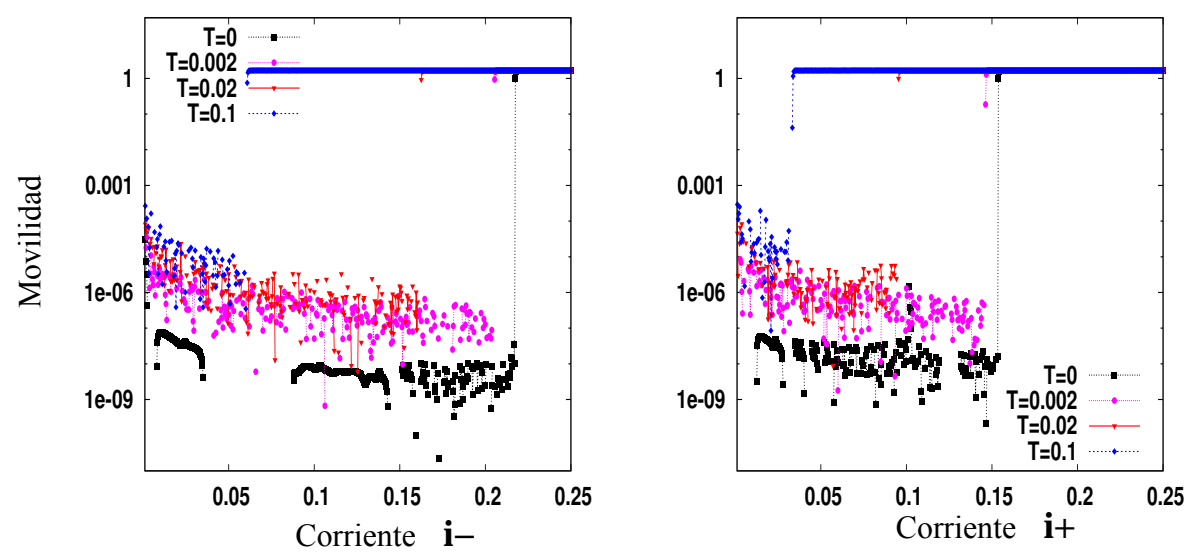

Figura 5.3: Curva de movilidad del fluxón en un anillo ratchet de 9 uniones $\lambda=0.4$ y $\Gamma=0.001$, dos direcciones de la corriente y 4 diferentes temperaturas $(T=0,0.002$, 0.02 y 0.1$)$.

esta forma, lo que facilita la comparación de la dinámica en uno y otro sentido de la corriente.

Las curvas de movilidad, como la dibujada en la figura 5.3 nos posibilitan establecer los criterios de movilidad crítica para realizar la estadística de la fuerza de desanclaje contra la temperatura. En la figura 5.3 se ha dibujado la corriente contra movilidad para un caso de amortiguamiento bajo $\Gamma=0.001$. A la izquierda de la figura5.3, se presenta la movilidad para corriente $i+$, y a la derecha con $i-$. Se aprecia que la dinámica es muy similar, salvo en el valor de la corriente de desanclaje para las diferentes temperaturas.

La figura 5.4 muestra curvas $I$ - $V$ para un fluxón en un arreglo de 9 uniones con $\lambda=0.4$. La simulación está hecha para tres valores del amortiguamiento y 4 valores de la temperatura. La corriente se incrementa desde cero hasta un valor máximo en el que se aprecia la rama óhmica, la rampa promedio fue de $\frac{8}{3} \times 10^{-7}$ en unidades normalizadas. Hemos dibujado ambos sentidos de la corriente en la misma ubicación del dibujo, con el fin de comparar sus valores de desanclaje en el cambio de estado del fluxón de velocidad cero a un estado de velocidad positiva en el caso de corriente $i+$ y velocidad negativa para $i-$.

Observemos en las figuras el comportamiento a temperatura cero. Para $\lambda=0.4$ la corriente de desanclaje en el caso de $i+$ es de aproximadamente $i_{\text {dep }}^{+}=0.154$. Cuando la corriente es negativa se obtiene $i_{\text {dep }}^{-}=-0.217$. Un detalle mejor del depinning se observa en la parte inferior de la figura 5.4 en donde se ha realizado una ampliación de la misma a bajos valores del voltaje. Como en el caso de la red regular,también se observa que con el incremento de la temperatura, la corriente necesaria para el desanclaje, va disminuyendo. 

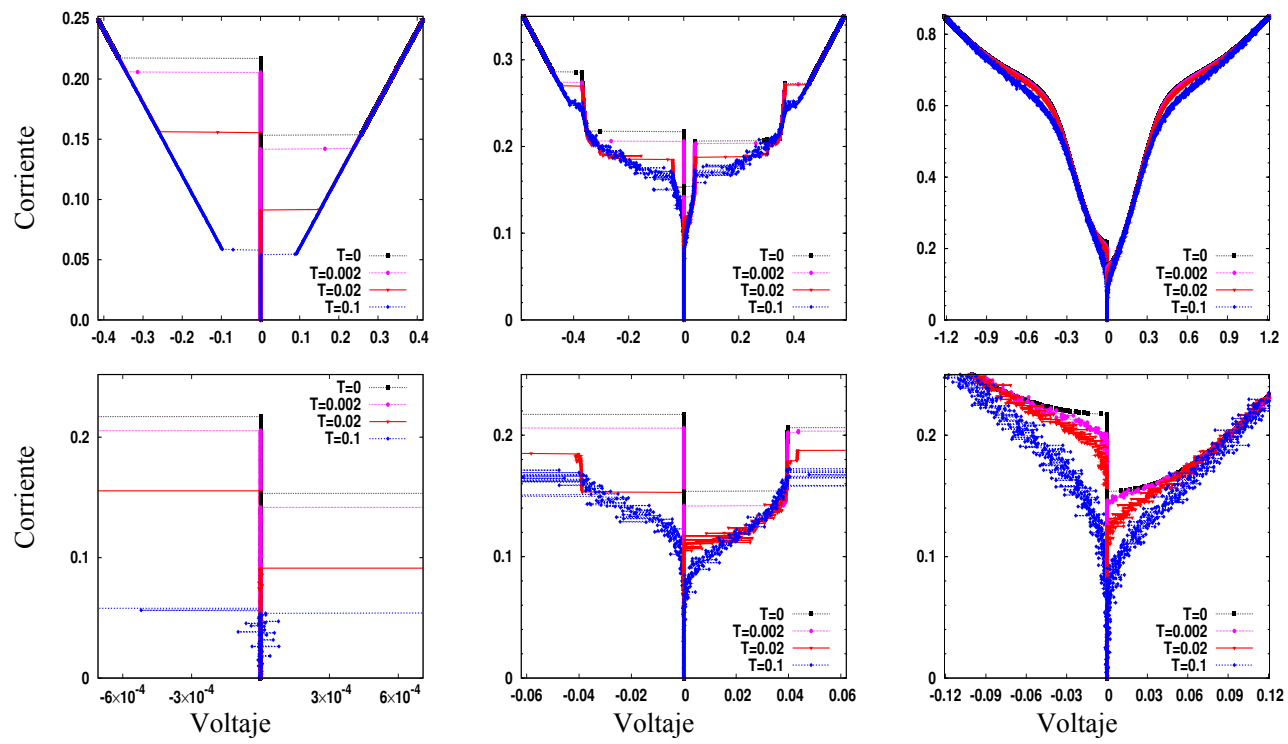

Figura 5.4: Curvas $|i|-v$ para un fluxón en un anillo ratchet de 9 uniones $\lambda=0.4$ a $\Gamma=0.01$ (izquierda), $\Gamma=0.1$ (medio) y $\Gamma=1.0$ (derecha). Cada figura muestra 4 diferentes temperaturas $(T=0,0.002,0.02$ y 0.1$)$.

La forma de las curvas características $I$ - $V$ cambian si aumentamos el valor del amortiguamiento. Así la figura de bajo amortiguamiento (izquierda) sólo muestra transición del estado del voltaje cero a la rama óhmica del sistema. La figura central muestra los resultados para el caso de $\Gamma=0.1$. En ese caso se puede apreciar la zona de bajo voltaje que se corresponde a las resonancias del fluxón. Para $\Gamma=1,0$ nos acercamos a la curva sobre amortiguada redondeada por la acción de las fluctuaciones térmicas.

En todos los casos se observa que al aumentar la temperatura la asimetría de la curva $I-V$ se reduce. Esto es fácil de entender ya que a altas temperaturas los detalles del potencial quedan apantallados por la intensidad de las fluctuaciones térmicas.

Un hecho notable es que en general el sistema de 9 uniones con 1 fluxón en configuración ratchet, con acoplamiento $\lambda=0.4$ y $\Gamma=0.01$ no presenta región de difusión de bajo voltaje, aún a temperaturas altas como en el caso $T=0.1$. Por lo tanto en este caso sólo tiene sentido definir una corriente crítica, $i_{\text {dep }}$, que marca la transición desde el estado de voltaje cero hasta la rama óhmica; observándose eso sí, que en el caso de la temperatura $T=0.1$ el fluxón fluctúa bastante desde su posición de equilibrio. Con amortiguamientos más altos la dinámica muestra los escalones debidos a la resonancia y la difusión debida a la temperatura. 

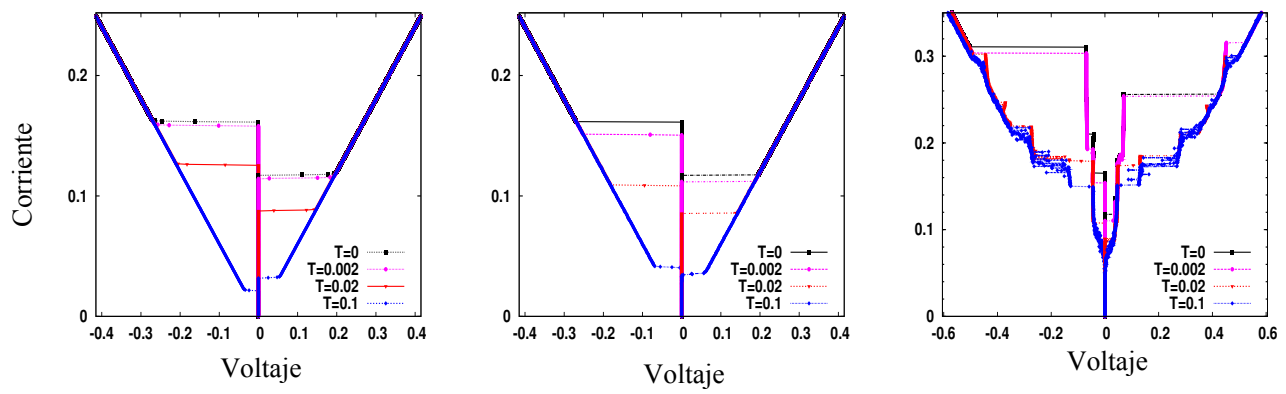

Figura 5.5: Curvas $|i|-v$ para un fluxón en un anillo ratchet de 9 uniones $\lambda=0.6$ a $\Gamma=0.001$ (izquierda), $\Gamma=0.01$ (medio) y $\Gamma=0.1$ (derecha). Cada figura muestra 4 diferentes temperaturas $(T=0,0.002,0.02$ y 0.1$)$.
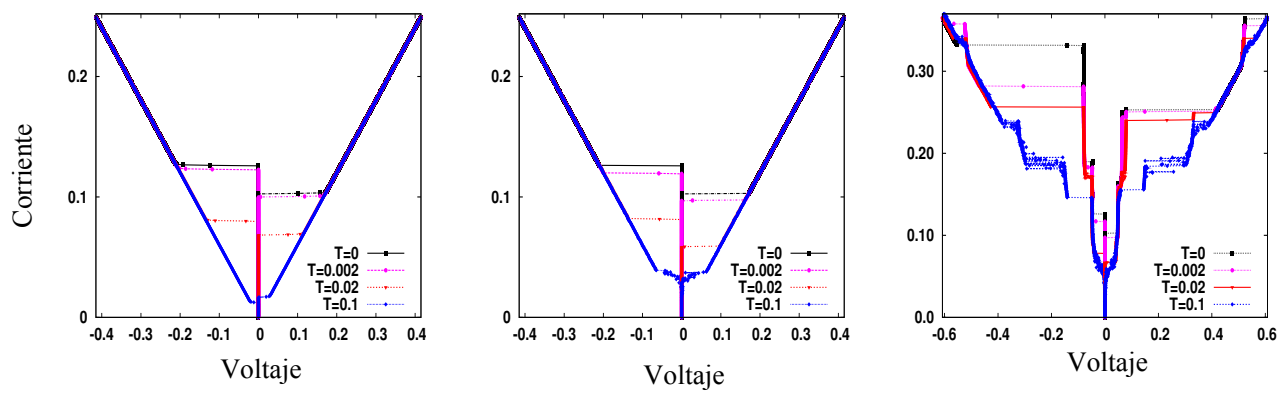

Figura 5.6: Curvas $|i|-v$ para un fluxón en un anillo ratchet de 9 uniones $\lambda=0.8$ a $\Gamma=0.001$ (izquierda), $\Gamma=0.01$ (medio) y $\Gamma=0.1$ (derecha). Cada figura muestra 5 diferentes temperaturas ( $T=0,0.002,0.02$ y 0.1$)$.

La ausencia de una rama de difusión en la dinámica del sistema es un hecho a primera vista sorprendente si se compara con los resultados obtenidos en el caso de una red regular, donde dicho estado de bajo voltaje aparece a valores pequeños del amortiguamiento y temperaturas suficientemente altas. No obstante si recordamos lo visto en el capítulo 4 la aparición de la rama de difusión dependía de manera importante del tamaño del sistema y era muy evidente para anillos grandes y no tanto para anillos de tamaño menor. En particular en nuestro caso nuestro anillo tiene 9 uniones pero el potencial efectivo para el fluxón consta de tan sólo 3 periodos. Creemos que este es el motivo por el cual no se aprecia rama de difusión en nuestras simulaciones. Si este argumento es correcto, esta rama estaría presente en resultados sobre redes mayores.

A continuación estudiamos el sistema en configuración ratchet con valores del acoplamiento mayores al estudiado anteriormente. La figura 5.5 muestra los resultados para $\lambda=0.6$ y la figura 5.6 para $\lambda=0.8$. En todos los casos se presentan tres valores del amortiguamiento, $\Gamma=0.01$ (izquiera), $\Gamma=0.1$ (cen- 
tro) y $\Gamma=1.0$ (derecha). Se observa que la asimetría entre las dos direcciones de la corriente afecta fundamental a la corriente de desanclaje y se reduce fuertemente al aumentar la temperatura.

En la tabla5.1 se consigna la diferencia entre las corrientes de escape para las dos direcciones de la corriente en el sistema ratchet a $T=0$. Al aumentar la temperatura, en las figuras $5.4,5.5$ y 5.6 puede observarse que a bajas temperaturas la diferencia entre la corriente de escape en las dos direcciones es significativamente mayor que cuando la temperatura aumenta. No obstante, esta diferencia se mantiene relativamente constante al variar el amortiguamiento. Los detalles sobre la evolución de las corrientes de desanclaje con la temperatura y el amortiguamiento, serán presentados en la siguiente sección.

\begin{tabular}{|c||c|c|c|c|}
\hline$\lambda=$ & $i_{\text {dep }}^{+}(T=0)$ & $i_{\text {dep }}^{-}(T=0)$ & $\Delta i_{\text {dep }}$ & $\Delta i_{\text {dep }} / i_{\text {dep }}^{\text {med }}$ \\
\hline \hline 0.4 & 0.154 & -0.217 & 0.063 & 0.34 \\
\hline 0.6 & 0.118 & -0.162 & 0.044 & 0.31 \\
\hline 0.8 & 0.103 & -0.127 & 0.024 & 0.21 \\
\hline
\end{tabular}

Cuadro 5.1: Asimetría en el depinning de un fluxón en una red ratchet a $T=0$. $\Delta i_{\text {dep }}=\left|i_{\text {dep }}^{+}+i_{\text {dep }}^{-}\right|$y $i_{\text {dep }}^{\text {med }}=\frac{1}{2}\left(\left|i_{\text {dep }}^{+}\right|+\left|i_{\text {dep }}^{-}\right|\right)$.

\subsection{2. $\left\langle i_{\text {dep }}(T)\right\rangle$}

En esta sección presentaremos los resultados para el cálculo de la corriente de depinning media de un fluxón en un red ratchet como función de la temperatura. Compararemos los resultados obtenidos para corrientes positivas y negativas a varios valores del amortiguamiento $(\Gamma=0.001$ y $\Gamma=0.01)$ y el acoplamiento $\lambda=0.4, \lambda=0.6$ y $\lambda=0.8$ e intentaremos entender los resultados obtenidos en un marco común.

Como hemos visto en la sección anterior, para los valores de parámetros seleccionados la curva $I$ - $V$ del sistema experimenta un paso de la rama de voltaje 0 a la rama óhmica de alto voltaje sin pasar por estadios intermedios de bajo voltaje, ya sean resonancias o difusión.

La figura 5.7 recoge el resultado de la simulaciones. En la figura superior se muestra $\left|\left\langle i_{\text {dep }}\right\rangle\right|$ para corrientes positiva (símbolos huecos) y negativas (símbolos rellenos). La figura inferior muestra la desviación estándar obtenida en cada caso. El comportamiento observado es cualitativamente similar al obtenido para redes regulares con la salvedad de que ahora debemos considerar las dos polaridades de la corriente externa.

Resulta interesante comparar la evolución del grado de asimetría del siste- 

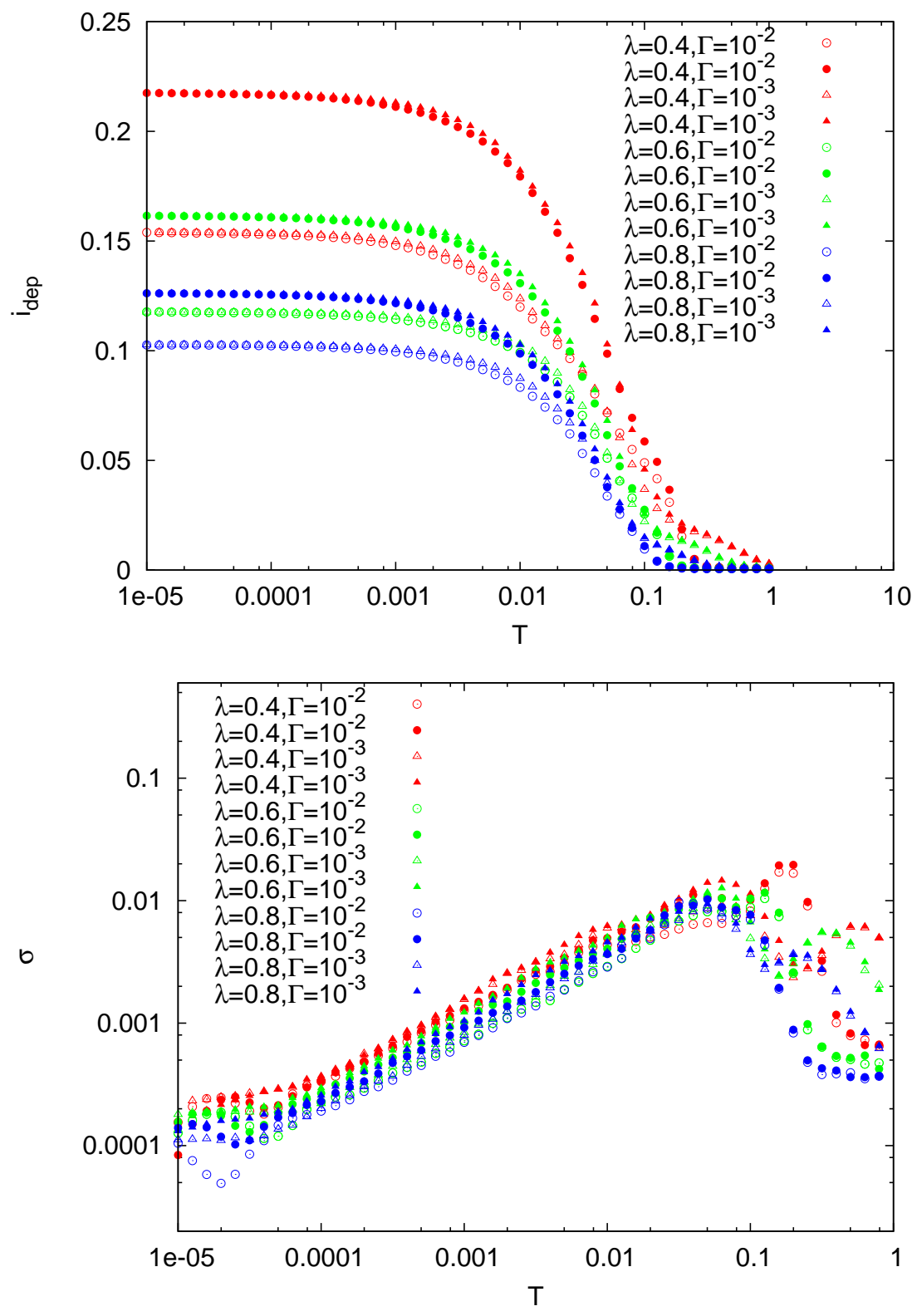

Figura 5.7: Figura superior: Cálculo del valor medio de la corriente de depinning de un fluxón en una red ratchet con 9 uniones para $\lambda=0.4$ (rojo), $\lambda=0.6$ (verde) y $\lambda=0.8$ (azul). Se muestran los resultados para dos valores del amortiguamiento $\Gamma=0.01$ (círculos) y $\Gamma=0.001$ (triángulos). Se muestra tanto $i_{d e p}^{+}$, la corriente de depinning positiva (símbolos huecos) como $-i_{d e p}^{-}$, el valor absoluto de la negativa (símbolos llenos). Los promedios se han realizado sobre 1000 realizaciones. Figura inferior: muestra el valor de la desviación estándar $\sigma$ para los resultados de la simulación anterior. 


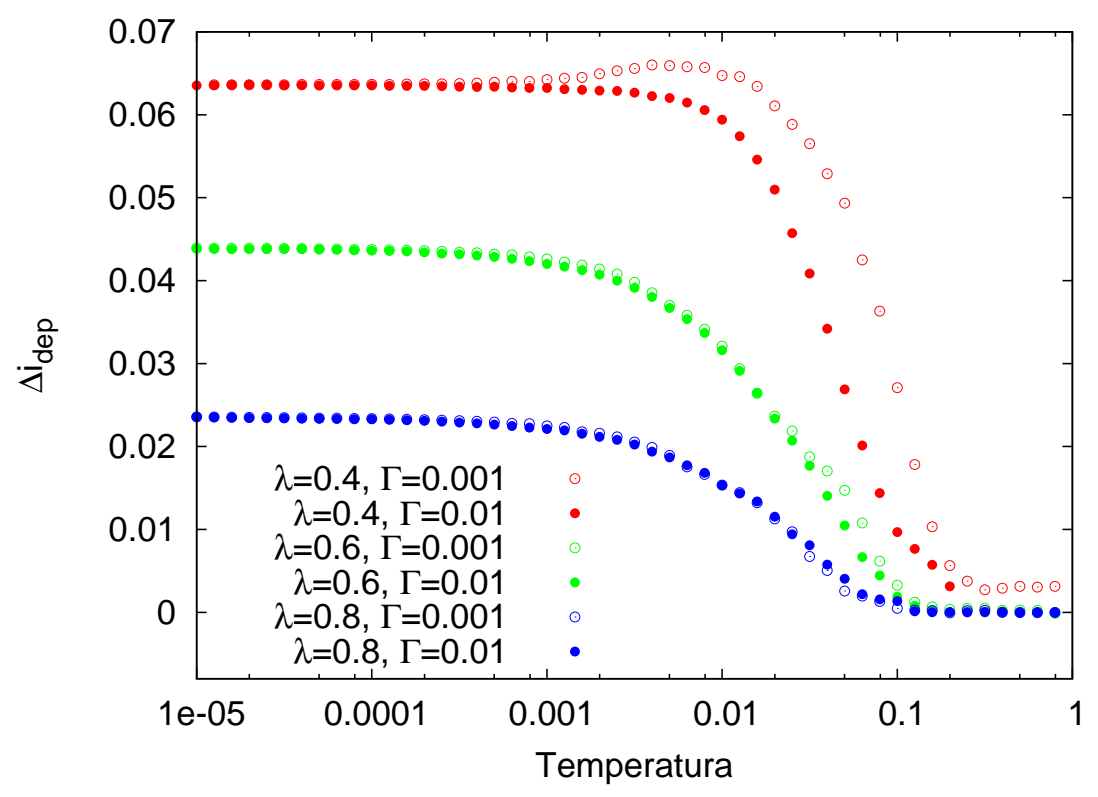

Figura 5.8: La figura muestra el valor de $\Delta i_{d e p}$ definido como $\Delta i_{d e p}=-\left(i_{d e p}^{+}+i_{d e p}^{-}\right)$ como función de la temperatura para los distintos valores de $\lambda$ y $\Gamma$ calculados.

ma. Para ello definimos $\Delta i_{\text {dep }}=\left|i_{\text {dep }}^{-}\right|-\left|i_{\text {dep }}^{+}\right|$. Los resultados se muestran en la figura 5.8. Como puede observarse, para $\lambda=0.6$ y $\lambda=0.8$ esta medida decrece monotonamente con la temperatura y no se aprecian diferencias entre los dos valores de amortiguamiento estudiados. Sin embargo, el caso $\lambda=0.4$ no es tan sencillo ya que el parámetro $\Delta i_{d e p}$ alcanza un máximo para $T \sim 0.01$. Además, por encima de $T=0.001$, cuando ese máximo comienza a desarrollarse, se observan importantes diferencias entre los casos $\Gamma=0.001$ y $\Gamma=0.01$. Estos resultados confirman las impresiones avanzadas a partir de la observación de las curvas $I$ - $V$ del sistema.

Podemos intentar entender dentro de un mismo marco común el conjunto de resultados presentados en la figura 5.7. Con respecto el eje $y$, esto puede lograrse si se normalizan los valores de la corriente de depinning obtenidos por el resultado de temperatura nula y se representa $\left\langle i_{d e p}(T)\right\rangle / i_{\text {dep }}^{0}$. Con respecto al eje $x$, resulta natural normalizar todas las temperaturas con respecto al valor de la barrera a $i=9$ (ver tabla 5.2) y representar $T / E_{P N}$. La figura 5.9 muestra un muy buen acuerdo entre las 12 curvas representadas, siendo el acuerdo excelente para los casos $\lambda=0.6$ y $\lambda=0.8$ y detectándose pequeñas diferencias para $\lambda=0.4$. Estas pequeñas diferencias serían esperables a tenor de lo expuesto en páginas anteriores.

Finalmente, de modo similar a como se hizo para el caso de una red re- 

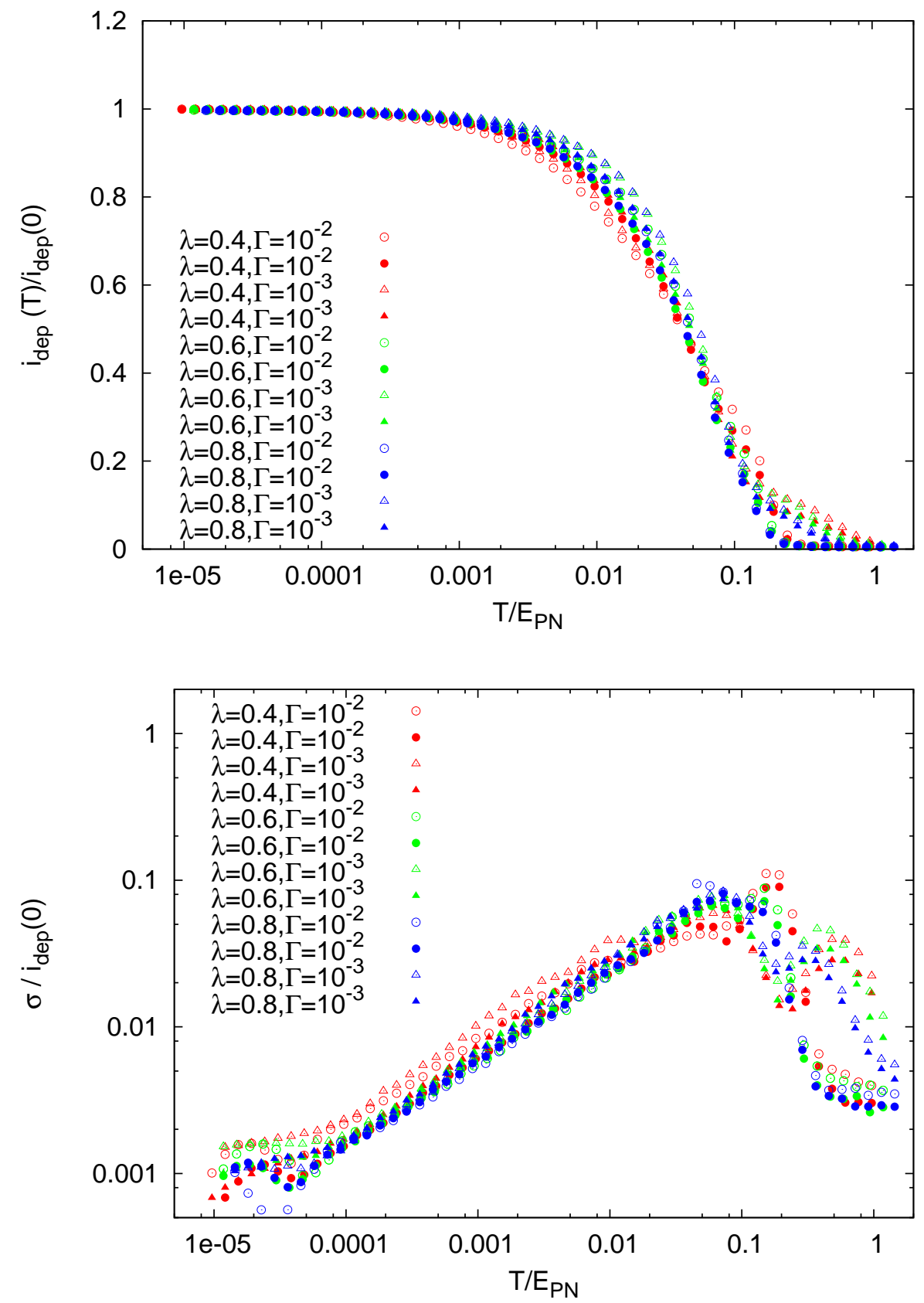

Figura 5.9: La figura representa los mismos resultados mostrados en la figura 5.7 pero normalizando la corriente de desanclaje por su valor a temperatura cero y la temperatura por el valor de la barrera de Peierls-Nabarro al acoplamiento correspondiente. Esto indica que los resultados obtenidos pueden entenderse en un marco común. 


\begin{tabular}{|c||c|c||c|c|c|}
\hline & $i_{\text {dep }}^{+}$ & $i_{\text {dep }}^{-}$ & $E_{P N}$ & $\omega_{P N}$ & $m$ \\
\hline \hline$\lambda=0.4$ & 0.154 & -0.2177 & 1.038 & 1.806 & 0.22 \\
\hline$\lambda=0.6$ & 0.118 & -0.1616 & 0.8494 & 1.5461 & 0.175 \\
\hline$\lambda=0.8$ & 0.103 & -0.1265 & 0.6944 & 1.402 & 0.155 \\
\hline
\end{tabular}

Cuadro 5.2: Parámetros característicos de 1 fluxón en una red ratchet de 9 uniones

gular con un fluxón, podemos intentar entender los resultados obtenidos en el marco de la imagen del fluxón como una partícula que se mueve en un potencial efectivo, que en este caso será un potencial ratchet. El capítulo 4 valido la aproximación de Bütikker, Harris y Landauer (BHL) [36] al cálculo del valor medio de la corriente de escape del fluxón en gran parte del rango de temperaturas y acoplamiento estudiados. Este resultado esta basado en la consideración del escape de una partícula en un potencial cúbico cuya barrera cambia con la corriente externa. En el capítulo 3 hemos visto que al incluir corriente la aproximación de potencial cúbico es también válida para potenciales ratchet inclinados. Por todo ello decidimos calcular numéricamente la predicción BHL para el escape de fluxón y su desviación estándar donde los parámetros del fluxón entran en la definición de las corrientes críticas y los valores de las barreras y frecuencias a corriente cero:

$$
\begin{gathered}
\Delta U=E_{P N}\left[\left(1-f^{2}\right)^{1 / 2}-f \arccos f\right] \\
\omega_{a}=\omega_{P N}\left(1-f^{2}\right)^{1 / 4}
\end{gathered}
$$

donde $f=i / i_{d e p}^{0}$. Recordamos que el otro parámetro importante en la teoría es la tasa de variación de la corriente aplicada o rampa.

La tabla 5.2 muestra los parámetros fundamentales (donde hemos añadido la masa efectiva $m$ aunque este parámetro no entra en la teoría).

Las figuras 5.10, 5.11,5.12 recogen los resultados. Podemos ver que las predicciones teóricas concuerdan razonablemente bien con las simulaciones realizadas en los casos de mayor acoplamiento, $\lambda=0.6$ y $\lambda=0.8$. Sin embargo, de nuevo, en el caso $\lambda=0.4$ el acuerdo no es satisfactorio. Estas diferencias son debidas al papel que juegan los grados de libertad adicionales (los fonones) en la dinámica del sistema. Como se vió en el estudio de las redes regulares, este papel es mayor cuando menor es el parámetro de acoplamiento $\lambda$, que equivale a una mayor importancia del carácter discreto del sistema. 

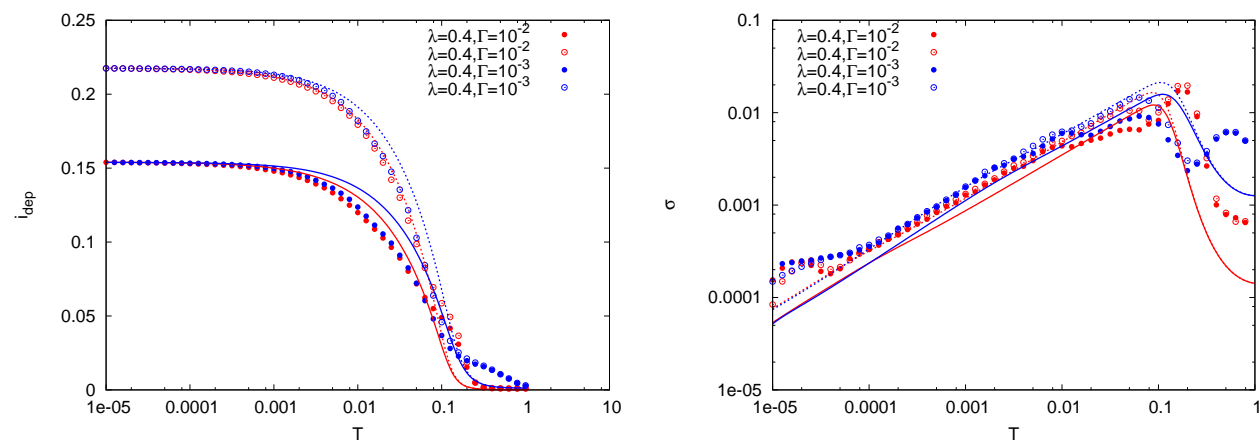

Figura 5.10: Comparación de los resultados obtenidos para un fluxón en una red ratchet a $\lambda=0.4$ y las predicciones de la teoría BHL de escape de una partícula en un potencial metaestable.
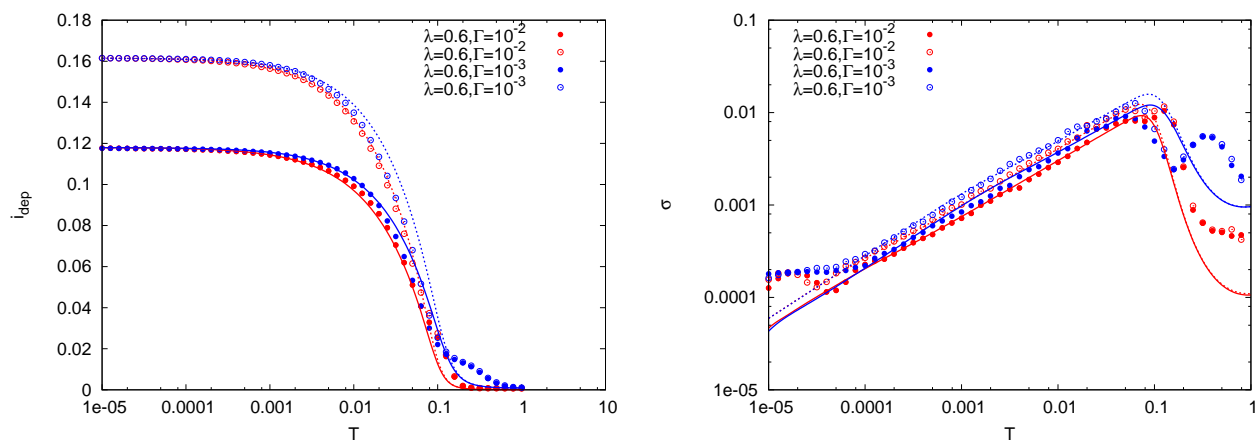

Figura 5.11: Comparación de los resultados obtenidos para un fluxón en una red ratchet a $\lambda=0.6$ y las predicciones de la teoría BHL de escape de una partícula en un potencial metaestable.
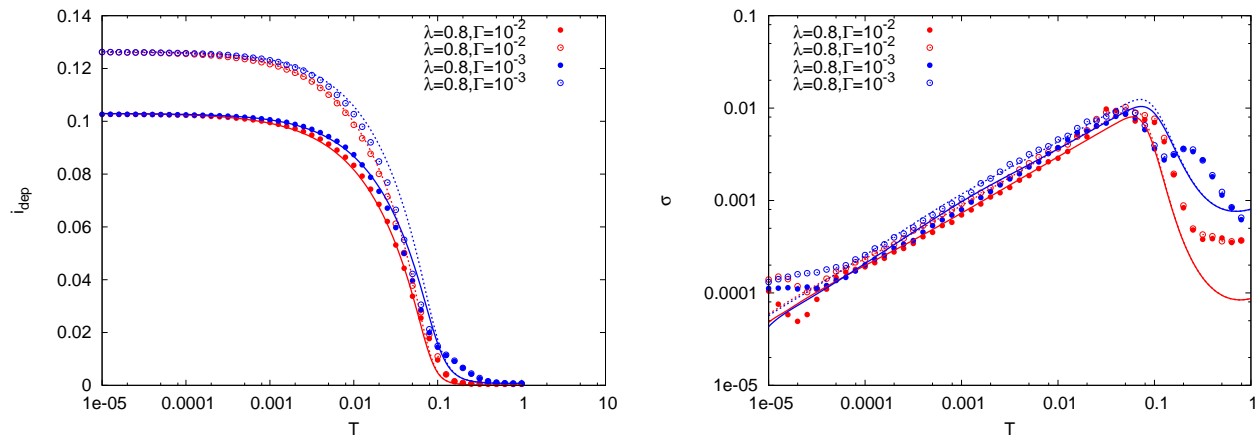

Figura 5.12: Comparación de los resultados obtenidos para un fluxón en una red ratchet a $\lambda=0.8$ y las predicciones de la teoría BHL de escape de una partícula en un potencial metaestable. 


\subsection{Red ratchet con 9 uniones y dos fluxones}

Como en el caso de la red regular se realizó el estudio del comportamiento dinámico de 2 fluxones en una red ratchet de 9 uniones. Comenzaremos nuestro estudio con la dinámica en las curvas $I$ - $V$ del sistema. Se usarán los parámetros de acoplamiento $\lambda=0.4,0.6$ y 0.8 a diferentes amortiguamientos y valores de la temperatura. Finalmente se presentaran las curvas del valor medio de la corriente de escape en función de la temperatura

\subsubsection{Curvas $I-V$}

Las figuras $5.13,5.14$ y 5.15 muestra la dinámica de una red ratchet con los valores de acoplamiento y amortiguamiento, especificados en cada caso. Se puede ver que existe diferencia significativa entre las corrientes de escape de ambos sentidos de la corriente, ver tabla 5.3, y que en el régimen subamortiguado $\Gamma=0.001$ y $\Gamma=0.01$ no hay presencia de difusión.

Así, en el sistema ratchet con $\lambda=0.4$, la dinámica $I$ - $V$ para el sector subamortiguado; $\Gamma=0.001$ y $\Gamma=0.01$ es similar al caso del sistema con 1 fluxón. También está presente el efecto ratchet manifestado ahora por la diferencia en los valores de la corriente de depinning consignados en la tabla 5.3. Con un valor mayor del amortiguamiento $\Gamma=0.1$ hay presencia de difusión a alta temperatura y multiestados representados en los escalones de resonancias. Una situación similar a la anterior, ocurre $\lambda=0.6$ en el régimen subamortiguado. Con $\lambda=0.6, \Gamma=0.1$ desde $T=0.02$ y mayores, se presenta difusión y multiestados del kink. Con temperaturas más altas la zona de difusión se vé extendida hasta alcanzar la región lineal. En la dirección $i$ - se puede ver que sin ruido térmico el kink escapa y comienza a moverse, pasando por estados de resonancia que forman escalones previos al salto a la rama óhmica. A medida que aumenta la temperatura, el kink no define una corriente de escape y en su lugar se presenta la conocida difusión que vá desde el estado de voltaje cero hasta la zona óhmica, lo dicho anteriormente se puede ver en la figura 5.14.

\begin{tabular}{|c||c|c|c|c|}
\hline$\lambda=$ & $i_{\text {dep }}^{+}(T=0)$ & $i_{\text {dep }}^{-}(T=0)$ & $\Delta i_{\text {dep }}$ & $\Delta i_{\text {dep }} / i_{\text {dep }}^{\text {med }}$ \\
\hline \hline 0.4 & 0.135 & -0.195 & 0.060 & 0.36 \\
\hline 0.6 & 0.096 & -0.130 & 0.034 & 0.30 \\
\hline 0.8 & 0.073 & -0.091 & 0.018 & 0.22 \\
\hline
\end{tabular}

Cuadro 5.3: Asimetría en el depinning de un fluxón en una red ratchet a $T=0$. $\Delta i_{\text {dep }}=\left|i_{\text {dep }}^{+}+i_{\text {dep }}^{-}\right|$y $i_{\text {dep }}^{\text {med }}=\frac{1}{2}\left(\left|i_{d e p}^{+}\right|+\left|i_{d e p}^{-}\right|\right)$. 

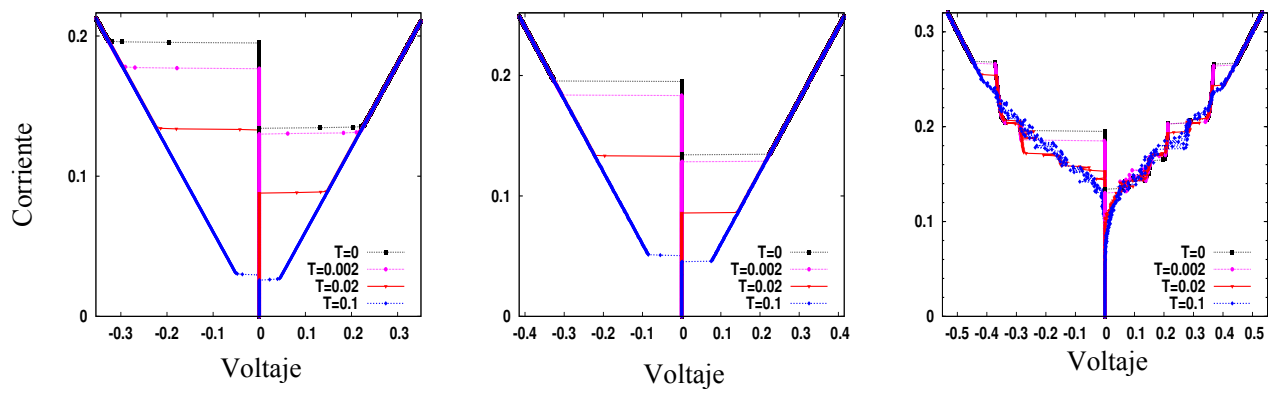

Figura 5.13: Curvas $|i|-v$ para dos fluxones en un anillo ratchet de 9 uniones. $\lambda=0.4$ y $\Gamma=0.001$ (izquierda), $\Gamma=0.01$ (medio) y $\Gamma=0.1$ (derecha). Cada figura muestra 4 temperaturas diferentes $(T=0,0.002,0.02$ y 0.1$)$.
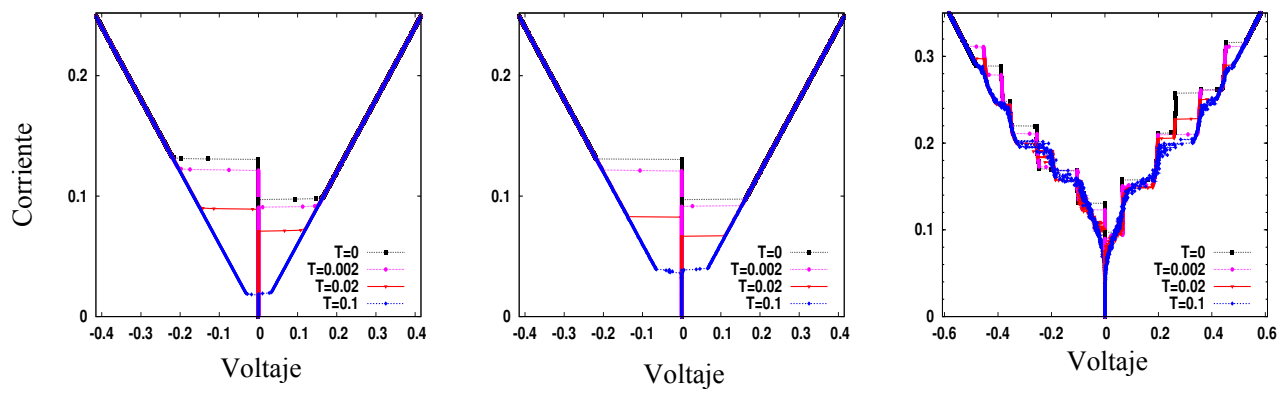

Figura 5.14: Curvas $|i|-v$ para dos fluxones en un anillo ratchet de 9 uniones. $\lambda=0.6$ y $\Gamma=0.001$ (izquierda), $\Gamma=0.01$ (medio) y $\Gamma=0.1$ (derecha). Cada figura muestra 4 temperaturas diferentes $(T=0,0.002,0.02$ y 0.1$)$.
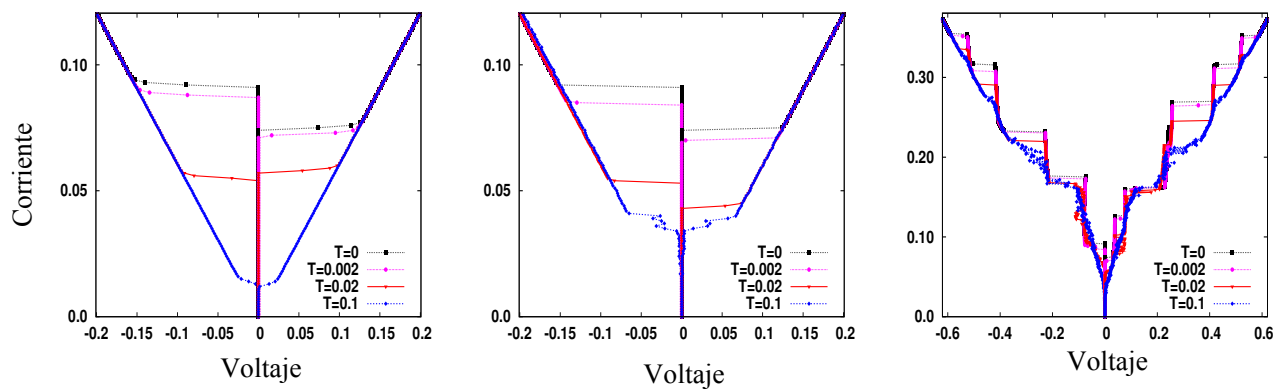

Figura 5.15: Curvas $|i|-v$ para dos fluxones en un anillo ratchet de 9 uniones. $\lambda=0.8$ y $\Gamma=0.001$ (izquierda), $\Gamma=0.01$ (medio) y $\Gamma=0.1$ (derecha). Cada figura muestra 4 temperaturas diferentes $(T=0,0.002,0.02$ y 0.1$)$.

Es importante destacar que en el caso $\lambda=0.8$, la dinámica para el sector subamortiguado es más interesante que en los casos anteriores de acoplamiento. Ahora podemos observar eventuales valores de voltaje no nulo previos al salto 

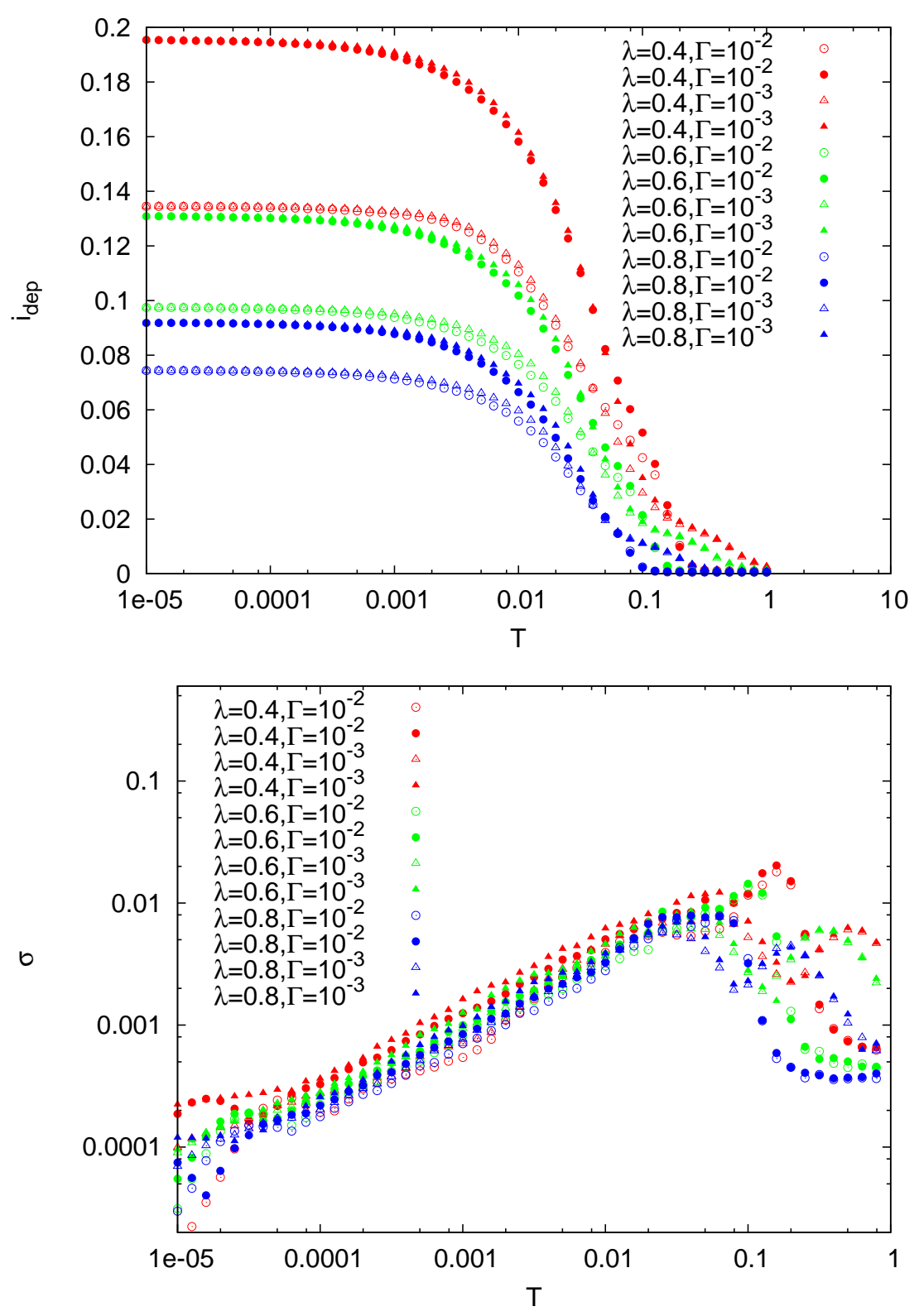

Figura 5.16: Figura superior: Cálculo del valor medio de la corriente de depinning de dos fluxones en una red ratchet con 9 uniones para $\lambda=0.4$ (rojo), $\lambda=0.6$ (verde) y $\lambda=0.8$ (azul). Se muestran los resultados para dos valores del amortiguamiento $\Gamma=0.01$ (círculos) у $\Gamma=0.001$ (triángulos). Se muestra tanto $i_{\text {dep }}^{+}$, la corriente de depinning positiva (símbolos huecos) como $-i_{d e p}^{-}$, el valor absoluto de la negativa (símbolos llenos). Los promedios se han realizado sobre 1000 realizaciones. Figura inferior: muestra el valor de la desviación estándar $\sigma$ para los resultados de la simulación anterior. 


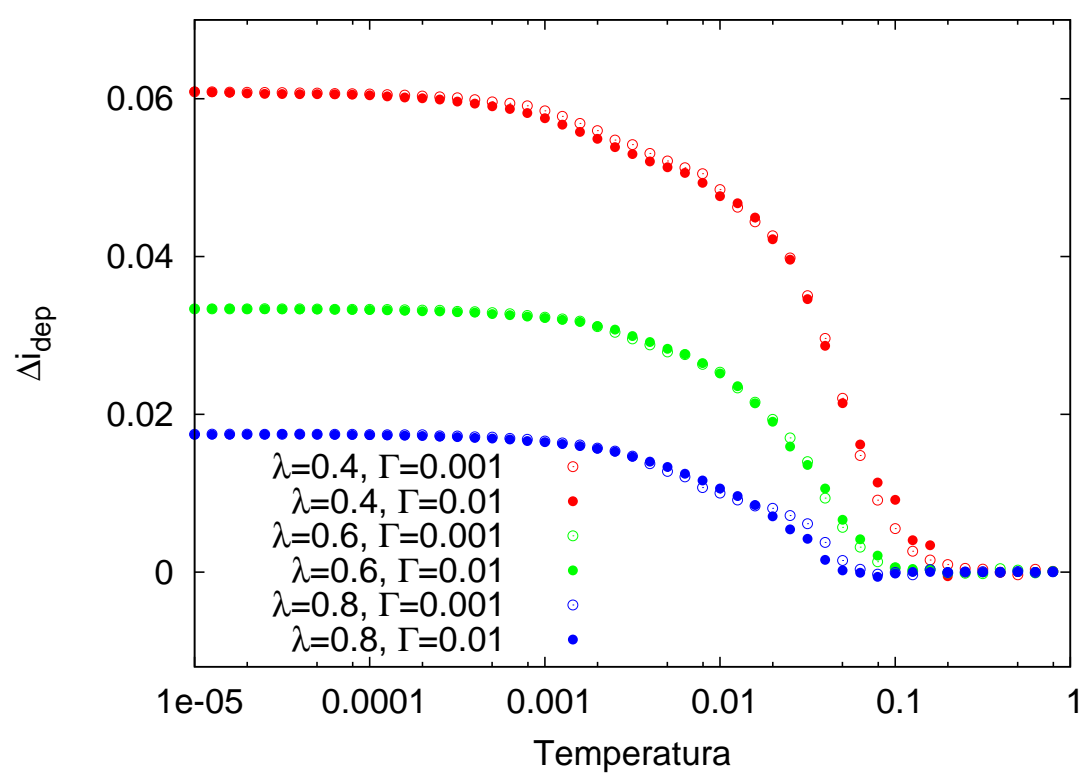

Figura 5.17: La figura muestra el valor de $\Delta i_{d e p}$ definido como $\Delta i_{d e p}=-\left(i_{d e p}^{+}+i_{d e p}^{-}\right)$ como fución de la temperatura para los distintos valores de $\lambda$ y $\Gamma$ calculados.

definitivo a la rama lineal de la curva. Esto es especialmente notable en las curvas para $\Gamma=0.01$ donde se manifiesta con claridad la presencia de la rama de difusión de bajo voltaje excitada a temperaturas suficientemente altas, en este caso para dos fluxones en el anillo. Dicha rama no ha aparecido en ninguna de las otras curvas $I-V$ presentadas, ni para un fluxón ni para dos. Con un valor mayor del amortiguamiento $\Gamma=0.1$ se observa la presencia de los escalones de resonancia del sistema que son suavizados por efecto térmico de manera especialmente notable a altas temperaturas, donde ya no se detectan discontinuidades importantes en la curva.

\subsection{2. $\left\langle i_{\mathrm{dep}}(T)\right\rangle$}

Finalmente, tal y como hicimos anteriormente cuando estudiamos el caso de un fluxón, procedemos a presentar los resultados obtenidos para la corriente de depinning media del sistema como función de la temperatura y para varios valores de los parámetros del sistema: $\lambda=0.4,0.6$ y 0.8 y $\Gamma=0.01$ y 0.001 en cada caso.

Los resultados obtenidos están mostrados en las figuras 5.16, 5.17 y 5.18 similares en cuanto a lo que representan que las figuras 5.7, 5.8 y 5.9 para el caso de un fluxón, mostradas en la sección anterior. En este caso cabe observarse menos anomalías para el caso de $\lambda=0.4$ que las descritas anteriormente 

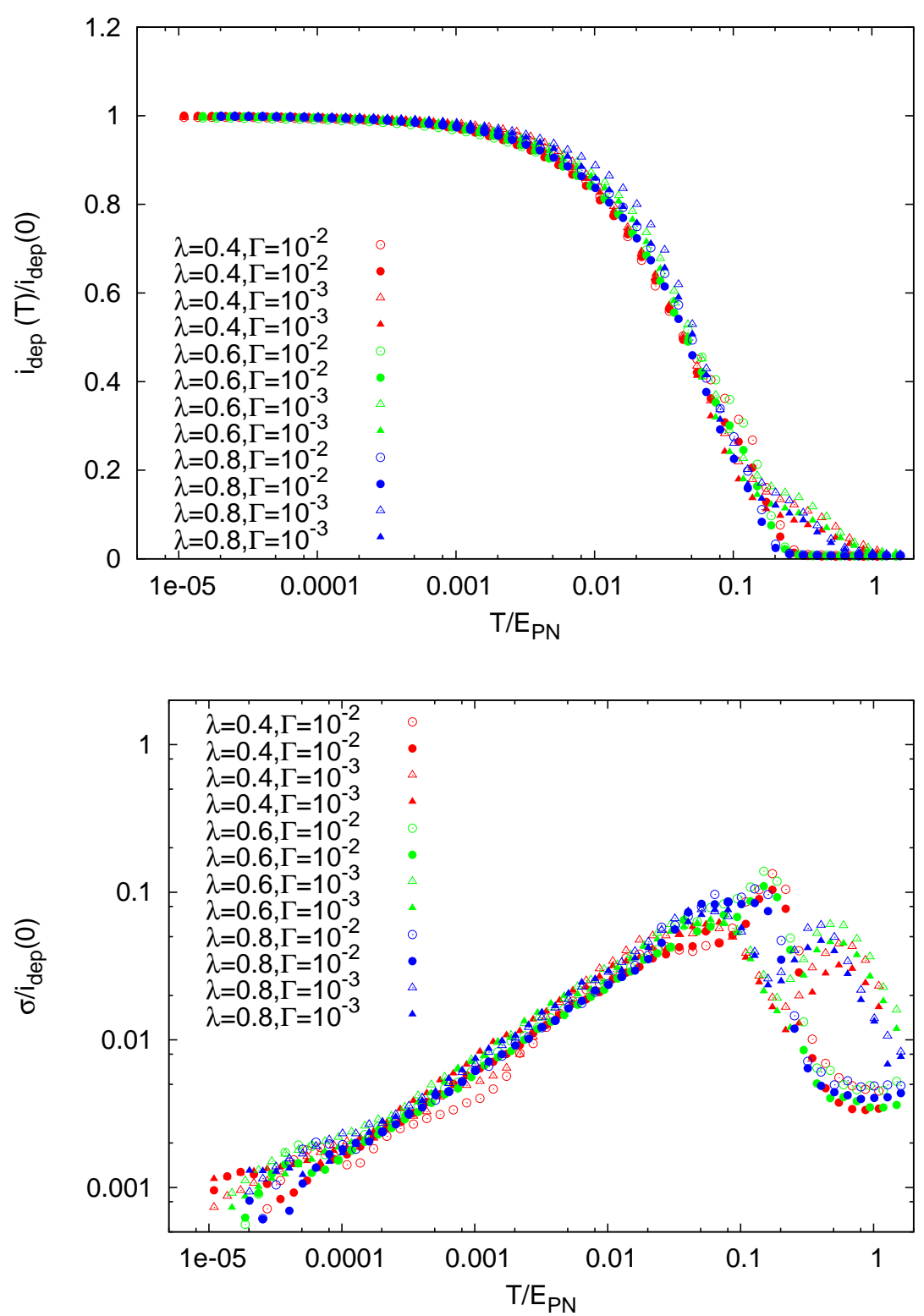

Figura 5.18: La figura representa los mismos resultados mostrados en la figura 5.16 pero normalizando la corriente de desanclaje por su valor a temepratura cero y la temperatura por el valor de la barrera de Peierls-Nabarro al acoplamiento correspondiente.

para un fluxón. En particular, se observa que la curva para $\Delta i_{\text {dep }}$ es monótona decreciente en todos los casos y muy similar para los dos valores del amortiguamiento estudiados. Muy notablemente, el acuerdo entre los distintos 
resultados (12 curvas) una vez normalizados es excelente en todos los casos tanto para el valor de $\left|\left\langle i_{\text {dep }}(T)\right\rangle\right|$ como para el valor de $\sigma(T)$ como puede verse en la figura 5.18. 



\section{Capítulo 6}

\section{Activación térmica en el régimen de amortiguamiento débil.}

En los capítulos anteriores hemos presentado resultados sobre el problema del depinning de fluxones en anillos superconductores. Siguiendo la imagen fluxón-partícula, los resultados han sido comparados con simulaciones de activación térmica de partículas individuales y con las predicciones teóricas. Dichas predicciones están basadas en la extensión llevada a cabo por Büttiker, Harris y Landauer [36] del resultado de Kramers para amortiguamiento débil al régimen de amortiguamiento débil-moderado.

Nuestros resultados nos indican que para ciertos valores de los parámetros la imagen es válida y los resultados de desanclaje del fluxón pueden ser entendidos completamente por la teoría de activación de partículas individuales. Sin embargo, como muestra la figura 4.12, a valores muy bajos del amortiguamiento encontramos que las simulaciones del kink o de la partícula se desvían de manera muy significativa de las predicciones de la teoría de Kramers.

Motivado por dicha observación, este capítulo lo hemos dedicado a intentar entender esta inesperada discrepancia y, en la medida de lo posible, elaborar una teoría que nos permita entender los resultados numéricos obtenidos. Para ello estudiamos el problema del escape térmico en el límite de bajo amortiguamiento. Veremos que en este régimen es crucial incorporar efectos de barrera finito a la teoría al es crucial para explicar los resultados de la activación térmica. Además, para entender los resultados de depinning, será necesario incorporar efectos de no equilibrio a las teorías a usuales. En el capítulo, proponemos una extensión teórica teniendo en cuenta los procesos de no-equilibrio, los cuales 

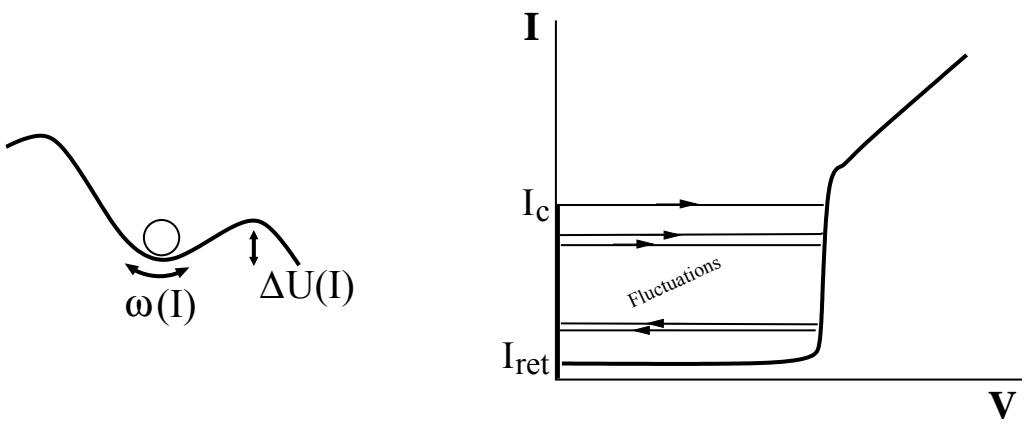

Figura 6.1: El problema de activación térmica de una unión Josephson puede ser formulado en términos del escape térmico de un partícula en un pozo metaestable.

se comparan excelentemente con las simulaciones.

\subsection{Teoría de Kramers estándar}

En 1940 Kramers derivó su famosa fórmula que describe las tasas de escape de una partícula Browniana en un pozo de potencial con el objetivo de calcular velocidades de reacción química [27]. Lejos de ser un caso particular, el escape activado por ruido es aplicable en un gran número de problemas en la ciencia, va desde la biología al procesamiento de información cuántica [102]. Debido a la variedad de campos involucrados, surgió una intensa actividad en el tema, proponiendo mejores y modernas teorías a un viejo problema [28, 29, 102].

El problema de activación térmica de una unión Josephson puede ser formulado en términos del escape térmico de un partícula en un pozo metaestable (figura 6.1). Por ello, los estudios sobre el switching térmico en las uniones Josephson (JJ) se benefician de este esfuerzo [103, 104, 24, 25, 32, 33, 34, 35]. Los resultados experimentales se ven afectados por las fluctuaciones térmicas y las mediciones en el laboratorio permiten predecir los parámetros de las uniones mediante el ajuste de los resultados obtenidos a las expresiones teóricas disponibles. Además, algunas tópicos fundamentales como la transición cuántica-clásica ha sido abordada a través de la medida de las tasas de escape $[32,33,34,23]$. Es claro, que tales mediciones deben compararse con los correspondientes resultados teóricos. No es necesario decir que la fórmula exacta no existe y que muchas teorías están disponibles en la literatura, que a partir del trabajo seminal de Kramers, cubren un conjunto diferente de parámetros $[28,29]$.

En una reciente trabajo numérico [105], para valores muy bajos del parámetro de amortiguamiento, se ha encontrado una desviación significativa de la 
corriente de switching de las uniones Josephson de los resultados esperados. Aquí presentamos una teoría que es capaz de dar cuenta de la desviación observada. Por otra parte, predice que este efecto se observará en cualquier sistema forzado, donde la relación entre el amortiguamiento y la rampa de fuerza no sea grande. En tal caso, las teorías usuales no son adecuadas, y, como veremos, es necesario incluir efectos de no equilibrio y la corrección de barrera finita en la descripción completa del problema.

Para precisar, la dinámica de la diferencia de fase en la unión se describen habitualmente con el, así llamado, modelo (RCSJ) resistivo, capacitivo y la unión en paralelo, que es equivalente al problema más general de una partícula browniana en un potencial metaestable:

$$
m \ddot{x}+m \gamma \dot{x}=-\frac{d V}{d x}+\xi(t),
$$

donde el potencial $V(x)=V_{0}(1-\cos x)-I x$ y $\xi(t)$ es la fuerza estocástica que describe las fluctuaciones térmicas. Consideramos aqui el ruido térmico blanco, $\langle\xi(t)\rangle=0$ y $\left\langle\xi(t) \xi\left(t^{\prime}\right)\right\rangle=2 m \gamma k_{B} T \delta\left(t-t^{\prime}\right)$.

Si definimos $f=I / V_{0}$, la altura de la barrera cambia al aumentar $I$ como

$$
\Delta U=2 V_{0}\left[\left(1-f^{2}\right)^{1 / 2}-f \arccos f\right]
$$

y la frecuencia $\omega_{a}(f)$ en el fondo del pozo está dada por

$$
\omega_{a}=\omega_{0}\left(1-f^{2}\right)^{1 / 4}
$$

donde $\omega_{0}=\left(V_{0} / m\right)^{1 / 2}$.

Para valores moderados a bajos del parámetro de amortiguamiento existe una corriente crítica (fuerza) dependiente de la temperatura para que el sistema pase de un estado de velocidad nula o superconductor $(\langle\dot{x}\rangle=0)$ a un estado resistivo $(m \gamma\langle\dot{x}\rangle=I)$. Esta situación se corresponde con el problema del escape de un pozo metaestable (figura 6.1). En experimentos de corriente de switching las curvas corriente-voltaje (fuerza-velocidad) se realizan para obtener la distribución de probabilidad de la corriente de switching, $P(I)$. Las distribuciones medidas puede estar directamente relacionadas con las tasas de activación térmica [25] y los resultados experimentales se pueden entender en términos de esos parámetros.

\subsubsection{Teoría de Kramers para amortiguamiento débil}

Para amortiguamiento muy débil, el resultado seminal de Kramers para la tasa de activación establece:

$$
r_{K L D}=\frac{\gamma J_{b}}{k_{\mathrm{B}} T} \times \frac{\omega_{a}}{2 \pi} \mathrm{e}^{-\Delta U / k_{B} T} .
$$




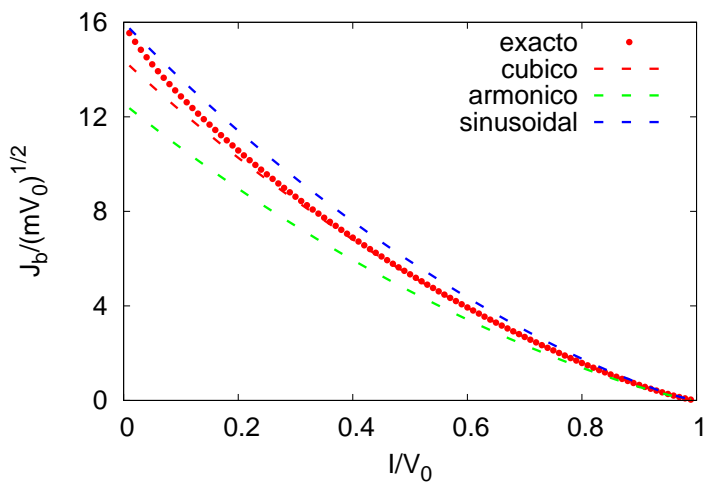

Figura 6.2: Acción en la barrera $J_{b} /\left(m V_{0}\right)^{1 / 2}$ como función de $I / V_{0}$ calculada de modo exacto, y comparación con los resultados para un potencial cúbico, armónico y coseno.

En esta expresión reconocemos el resultado de la teoría del estado de transición multiplicado por un prefactor válido en el régimen de amortiguamiento muy bajo.

En general, escribiremos

$$
r=k \times r_{T S T}=k \frac{\omega_{a}}{2 \pi} \mathrm{e}^{-\Delta U / k_{B} T} .
$$

donde $k$ es un prefactor y $r_{T S T}$ el resultado clásico de la llamada transitionstate-theory.

El resultado de Kramers establece entonces que en el límite de amortiguamiento muy pequeño, $\gamma \rightarrow 0$,

$$
k_{K L D}=\frac{\gamma J_{b}}{k_{\mathrm{B}} T}=\Delta
$$

$J_{b}$ representa el valor de la acción en la barrera. Para nuestro sistema se puede aproximar al resultado de un potencial cúbico $J_{b}=7.2 \Delta U / \omega_{a}$ (figura 6.2). Entonces:

$$
r_{K L D} \simeq \frac{7.2 \gamma}{2 \pi} \frac{\Delta U}{k_{\mathrm{B}} T} \mathrm{e}^{-\Delta U / k_{B} T}
$$

Esta ecuación muestra que la tasa escala de forma lineal con el amortiguamiento y sólo depende del cociente entre la barrera de energía y la energía térmica: $\Delta U / k_{B} T$. Esta expresión es válida solamente para amortiguamiento muy pequeño y el límite de la barrera infinita $\left(\Delta=\gamma J_{b} / k_{\mathrm{B}} T \ll 1\right.$ y $\left.\Delta U / k_{\mathrm{B}} T \gg 1\right)$.

Muchas teorías han ampliado el resultado Kramers al régimen de amortiguamiento moderado a débil [36, 106, 107, 96, 28] siguiendo la aproximación de barrera infinita. Dada su simplicidad, el resultado de Büttiker et al. (BHL) [36] 
generalmente se aplica en la literatura de uniones Josephson. Correcciones de barrera finita se han estudiado en [108, 109]. Recientemente, Drozdov Hayashi (DH) proponen una nueva teoría no perturbativa en la altura de la barrera [110].

\subsubsection{Extensiones de la teoría de Kramers al régimen de amor- tiguamiento moderado-débil.}

A continuación dedicaremos unas líneas a resumir los principales resultados teóricos que pretenden extender el resultado de Kramers desde el régimen de amortiguamiento débil al régimen de amortiguamiento débil-moderado. En todos los casos las teorías se encuadran en el marco del límite de barrera infinita, definido como $\Delta U \gg k_{B} T$. En la figura 6.3 mostraremos una comparación estre estos resultados y el resultado seminal de Kramers.

\section{Teoría de Büttiker, Harris y Landauer}

En 1983 Büttiker, Harris y Landauer [36] presentaron un reputado trabajo donde se intenta extender el resultado de Kramers al régimen de amortiguamiento débil-moderado.

Ellos obtuvieron la siguiente expresión para el prefactor $k$,

$$
k_{B H L}=\frac{4 \alpha}{\left[(1+4 \alpha / \Delta)^{1 / 2}+1\right]^{2}}
$$

Donde $\alpha$ es un factor de corrección mayor que la unidad aunque cercano a 1. Ellos hicieron simulaciones numéricas y usaron $\alpha$ como un parámetro ajustable obteniendo el mejor ajuste para $\alpha=1$. No obstante, otros autores y diversas consideraciones señalan un valor diferente, $\alpha=1.47 .^{1}$

Usando como antes la aproximación cúbica para la acción obtenemos

$$
k_{\mathrm{BHL}}=\frac{4}{\left[\left(1+\omega_{a} k_{B} T / 1.8 \gamma \Delta U\right)^{1 / 2}+1\right]^{2}}
$$

que es la expresión introducida por Devoret et al [33] y ampliamente usada en la literatura de uniones Josephson.

Podemos calcular el valor de $k_{B H L}$ en el límite $\Delta \ll 1$. Entonces

$$
k_{B H L}(\Delta \ll 1) \simeq \Delta\left[1-\left(\frac{\Delta}{\alpha}\right)^{1 / 2}\right] \simeq \Delta=k_{K L D}
$$

\footnotetext{
${ }^{1}$ La teoría BHL coincide con los resultados de Melnikov a bajo amortiguamiento para $\alpha=1.47$ hasta segundo orden. A su vez las dos en primer orden reobtienen el resultado de Kramers.
} 

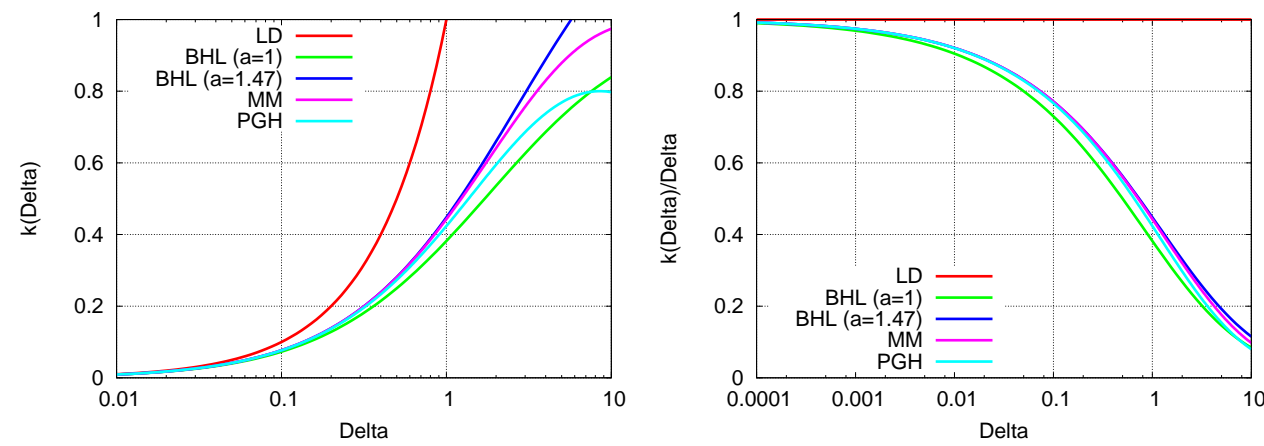

Figura 6.3: La figura compara los distintos resultados teóricos para el prefactor $k(\Delta)$ dados en el texto: $k_{K L D}, k_{B H L}(\alpha=1), k_{B H L}(\alpha=1.47), k_{M M}$ y $k_{P G H}$.

\section{Teoría de Mel'nikov y Meshkov}

En 1986 V. I. Mel'nikov y S. V. Meshkov (MM) publicaron un artículo titulado: Theory of activated rate processes: Exact solution of the Kramers problem [96]. En ese artículo ellos obtienen el siguiente valor para el prefactor de la teoría de activación térmica:

$$
\begin{aligned}
k_{M M}=A(\Delta) & =\exp \left\{\frac{1}{2 \pi} \int_{-\infty}^{+\infty} \ln \left[1-\exp \left(-\Delta\left(\lambda^{2}+1 / 4\right)\right)\right] \frac{d \lambda}{\lambda^{2}+1 / 4}\right\}= \\
& =\exp \left\{\frac{2}{\pi} \int_{0}^{\pi / 2} \ln \left[1-\exp \left(\Delta / 4 \cos ^{2} x\right) d x\right]\right\}
\end{aligned}
$$

expresión que debe ser evaluada numéricamente.

Con respecto el límite de bajo amortiguamiento, $\Delta \ll 1$ se obtiene

$$
k_{M M}=A(\Delta) \simeq \Delta-0.82 \Delta^{3 / 2}
$$

Esta ecuación coincide con el resultado de la teoría BHL para $\alpha=1.47$ (úsese 0.82371 en vez de 0.82) como también indicaron Risken and Voigtlaender [111].

\section{Teoría de Pollak, Grabert and Hänggi}

Grabert [106] y Pollak, Grabert y Hänggi [107] derivaron un resultado que es muy similar al de la teoría de MM

$$
k_{P G H}=k_{M H D} \times \exp \left[\frac{1}{\pi} \int_{-\infty}^{+\infty} \frac{d y}{1+y^{2}} \ln \left(1-e^{-\delta\left(1+y^{2}\right) / 4}\right)\right] .
$$

La principal diferencia radica en la definición de $\Delta$ y $\delta(\delta$ no se define de modo trivial en la aproximación PGH). Un segundo aspecto es que en la derivación 
aparece de manera natural el factor multiplicativo $k_{M H D}$. Este factor es el resultado de Kramers para el caso de moderado a alto amortiguamiento y está definido por:

$$
k_{M H D}=\frac{\left(\omega_{b}^{2}+\frac{1}{4} \gamma^{2}\right)^{1 / 2}-\frac{1}{2} \gamma}{\omega_{b}}=\left(1+\frac{\bar{\gamma}^{2}}{4}\right)^{1 / 2}-\frac{\bar{\gamma}}{2} ; \quad\left(\bar{\gamma}=\frac{\gamma}{\omega_{b}}\right)
$$

donde $\omega_{b}$ está asociado con la curvatura del potencial en la barrera: cerca de la barrera $U(q)=-\frac{1}{2} m \omega_{b}^{2} q^{2}$ mientras que cerca del mínimo metastable $U(q)=-\Delta U+\frac{1}{2} m \omega_{a}^{2}\left(q-q_{0}\right)^{2}$ (se ha situado la barrera en $q=0$ y el mínimo en $\left.q_{0}\right)$.

El parámetro $\delta$ se define como $\delta=\Delta E / k_{B} T$ con $\Delta E$ la energía media perdida del modo inestable perdida y su cálculo no es trivial.

Esta aproximación fue estudiada por Linkwitz y colaboradores [112] en 1992 que derivaron una expresión para el parámetro $\delta$ en el caso de un potencial cúbico

$$
\Delta E=\frac{36}{5} f \Delta U
$$

con

$f=\bar{\gamma} \kappa_{2}\left(1+\bar{\gamma}^{2} / 4\right)^{2}\left\{1+60 \bar{\gamma}\left(1+\bar{\gamma}^{2} / 4\right)^{1 / 2} k_{2}^{-8}\left[\psi^{\prime}\left(\kappa_{2}^{-2}\right)-\kappa_{2}^{2}-\kappa_{2}^{4} / 2-\kappa_{2}^{6} / 6\right]\right\}$, $\kappa_{2}=k_{M H D}, \bar{\gamma}=\gamma / \omega_{a}$ y $\psi^{\prime}(z)$ is la función trigamma ${ }^{2}$.

Para amortiguamiento débil

$$
f=\bar{\gamma}-1.804 \bar{\gamma}^{2}+O\left(\bar{\gamma}^{3}\right)
$$

Si comparamos los resultados de las teorías PGH y MM vemos que coinciden cuando el amortiguamiento es pequeño,entonces $f=\bar{\gamma}$ y $\delta=\Delta$.

\subsection{Correcciones de barrera finita}

Las expresiones dadas anteriormente son obtenidas en el marco de lo que se conoce como aproximación de barrera infinita $\Delta U \gg k_{B} T$. La experiencia demuestra que está aproximación es razonable en un gran rango de valores de los parámetros y se cumple de manera efectiva para valores del cociente barrera sobre temperatura tan bajos como 5. Sin embargo como vamos a demostrar a continuación los efectos de barrera finita son muy importantes en el rango de valores de amortiguamiento débil, que es el que nos interesa en este momento.

\footnotetext{
${ }^{2} \psi^{\prime}(z)=-\int_{0}^{1} \frac{x^{z-1} \ln x}{1-x} d x=\sum_{n=0}^{\infty} \frac{1}{(z+n)^{2}}$
} 
Con respecto a los experimentos de switching, en este límite el acoplamiento al baño térmico es muy débil y el tiempo para alcanzar el equilibrio térmico muy largo $(\sim 1 / \gamma)$. Este hecho tiene consecuencias importantes: (i) para sistemas forzados, el escape se produce a valores muy bajos de la razón $\Delta U / k_{B} T$ y (ii) las uniones pueden escapar antes de que el equilibrio térmico se establezca de tal forma que los efectos de no-equilibrio dominan el proceso. Para estudiar tales efectos es necesario conocer primero, la importancia del efecto barrera finita de activación de partículas a bajo amortiguamiento y tener en cuenta la energía media de las uniones antes de cada evento de switching.

A continuación resumiremos las dos teorías fundamentales que intenta incorporar los efectos de barrera finita:

\section{Resultado exacto en el límite de amortiguamiento nulo y barrera arbitraria}

En 1990, con motivo del 50 aniversario del trabajo original de Kramers, P. Hänggi, P. Talkner and M. Borkovec publicaron su célebre artículo de revisión [28] sobre el tema. En ese artículo encontramos una expresión que da cuenta del valor de la tasa de escape, para cualquier barrera y válida en el límite de amortiguamiento muy pequeño: $r(\gamma \rightarrow 0)=r_{H T B}$, con

$$
r_{H T B}=\gamma k_{B} T\left[\int_{0}^{J_{b}} d J \mathrm{e}^{-\beta E(J)} \int_{J}^{J_{b}} d J^{\prime} \frac{\omega\left(J^{\prime}\right)}{2 \pi} \frac{\mathrm{e}^{\beta E\left(J^{\prime}\right)}}{J^{\prime}}\right]^{-1},
$$

donde $J_{b}$ es la acción en la barrera y $\beta=1 / k_{B} T$. $E(J)$ y $\omega(J)$ son la expresión de la energía y frecuencia como función de la acción $J$.

Es interesante señalar que está misma expresión aparece en el marco del intento de Carmeli y Nitzan de encontrar una teoría válida en el régimen de débil-moderado amortiguamiento [113]

La ecuación mostrada es válida en lo que se conoce como energy-diffusion limit del problema y para barrera arbitraria. En el límite de amortiguamiento pequeño lo importante es el valor de la energía del sistema y la partícula escapa una vez que el tiene energía suficiente. La relajación de esa energía es lenta y no importa la posición de la partícula, si no la energía de la misma. Por ello el escape se produce mediante un proceso de difusión en la variable energía.

Si en la ecuación (6.19) se realiza la aproximación de barrera alta, se recupera el resultado de Kramers. Podríamos decir que se trata del equivalente al resultado de Kramers para cociente $\Delta U / k_{B} T$ arbitrario. 


\section{Teoría de Melnikov para las correcciones de barrera finita}

En un trabajo de 1993, Melnikov [108] refinó la teoría MM para incluir los efectos de barrera finita. En esta ocasión el prefactor $k$ viene dado por la siguiente expresión:

$$
k_{M F B}=A\left(\frac{\gamma}{\omega_{b}}, \frac{k_{B} T}{\Delta U}\right) \approx A_{0}(\Delta)-\frac{k_{B} T}{\Delta U} \frac{\Delta U}{\omega_{b} S_{b}}\left[A_{1}(\Delta) \ln \frac{\Delta U}{k_{B} T}+B_{1}(\Delta)\right]
$$

con $A_{0}(\Delta)=k_{M M}$ y $A_{1}(\Delta)$ y $B_{1}(\Delta)$ dos nuevas funciones a calcular.

El cálculo de esos dos nuevos coeficientes es bastante laborioso pero para $\Delta \ll 1$ se obtiene:

$$
\begin{gathered}
A_{0}(\Delta) \simeq \Delta-0.82 \Delta^{3 / 2} \\
A_{1}(\Delta) \simeq \Delta-1.63 \Delta^{3 / 2} \\
B_{1}(\Delta) \simeq \Delta\left(C_{U}+2+\ln 2-C\right)
\end{gathered}
$$

Ver que para la función $B_{1}(\Delta)$ sólo se tiene el término dominante en el desarrollo en $\Delta$. En esta última ecuación $C_{U}$ es un coeficiente que depende del potencial y $C$ el número de Euler $C=0.5772$.

En el caso de un potencial cúbico $\Delta U / \omega_{b} S_{b}=5 / 36$ y $C_{U}=3 \ln 6$, así $C_{U}+2+\ln 2-C=7.49$.

Si $\Delta \ll 1$ se obtiene las correcciones de barrera finita al resultado de Kramers para amortiguamiento débil. Usando $x=\Delta U / k_{B} T$, se tiene

$$
k_{L D M F B}=\Delta-\frac{\Delta}{7.2 x}(\ln x+7.49)
$$

y

$$
\frac{r_{L D M F B}}{\gamma}=\frac{7.2 x-\ln x-7.49}{2 \pi} \mathrm{e}^{-x}
$$

$\mathrm{O}$

$$
\frac{r_{\mathrm{LDMFB}}}{r_{\mathrm{LD}}}=1-\frac{\ln x}{7.2 x}-\frac{1.04}{x}
$$

Más tarde Ferrando y colaboradores [109] presentaron un estudio numérico del trabajo de Melnikov. En ese estudio puede encontrarse el cálculo de los coeficientes $A_{1}$ y $B_{1}$.

\section{Teoría de Drozdov y Hayashi}

Entre los años 1996 y 2000, Alexander N. Drozdov lideró una serie de trabajos sobre diversos aspectos de la teoría de Kramers. Algunos de sus resultados, como veremos, son muy notables, pero sorprendentemente sin embargo no han 
tenido el impacto esperado en la comunidad científica. En su trabajo de 1999 junto a Hayashi [110], se presenta un resultado teórico válido para cualquier valor de la barrera en el régimen de amortiguamiento desde muy pequeño a moderado.

La expresión de Drozdov se construye de la siguiente manera: Como de costumbre, la tasa de escape se expresa en función del resultado de la llamada transition-statete-theory y un factor de transmisión:

$$
r_{D H}=k_{D H} \times r_{T S T}^{\text {exacto }},
$$

donde se usa la siguiente definición para la tasa de escape de la transitionstate-theory: ${ }^{3}$.

$$
r_{T S T}^{\text {exact }}=\left\{\sqrt{2 \pi \beta m} \int_{-\infty}^{0} d x e^{-\beta V(x)}\right\}^{-1}
$$

$x=0$ señala la posición de la barrera, $V(x=0)=0$ y el escape ocurre de valores negativos a valores positivos $x$.

Hemos usado el superíndice "exacto" para distinguir esta expresión de la utilizada anteriormente (ver ecuación 6.5):

$$
r_{T S T}^{\text {aprox }}=\frac{\omega_{a}}{2 \pi} \mathrm{e}^{-\Delta U / k_{B} T} .
$$

$r_{T S T}^{\text {aprox }}$ puede derivarse de modo sencillo a partir del resultado para $r_{T S T}^{\text {exacto }}$ si se realiza la aproximación de "barrera parabólica":

$$
\int_{-\infty}^{0} d x e^{-\beta V(x)} \simeq \int_{-\infty}^{+\infty} d x e^{-\beta\left(-\Delta U+\frac{1}{2} m \omega_{a}^{2}\left(x-x_{0}\right)^{2}\right)}=\sqrt{\frac{2 \pi}{\beta m \omega_{a}^{2}}} e^{\beta \Delta U}
$$

La figura 6.4 evalúa la diferencia entre usar ambas expresioness (ecuaciones 6.28 y 6.29$)$.

Con respecto al factor multiplicativo $k_{D H}$, primero se da el valor del prefactor en el límite de amortiguamiento nulo, que resulta ser el apropiado para obtener el resultado exacto

$$
k_{D H}(\gamma \rightarrow 0)=k_{\mathrm{wc}}=r_{H T B} / r_{T S T}^{\text {exacto }}
$$

y así $r_{D H}(\gamma \rightarrow 0)=r_{H T B}$.

A partir de este resultado se propone un prefactor válido para valores mayores del amortiguamiento,

$$
k_{D H}=A\left(k_{\mathrm{wc}}\right)
$$

donde $A(x)$ es la función introducida por Mel'nikov y Meshkov, ecuación (6.11).

\footnotetext{
${ }^{3}$ Este resultado no es original de Drozdov, ya aparece en el trabajo seminal de Kramers [27] o en la recopilación de HTB [28]
} 

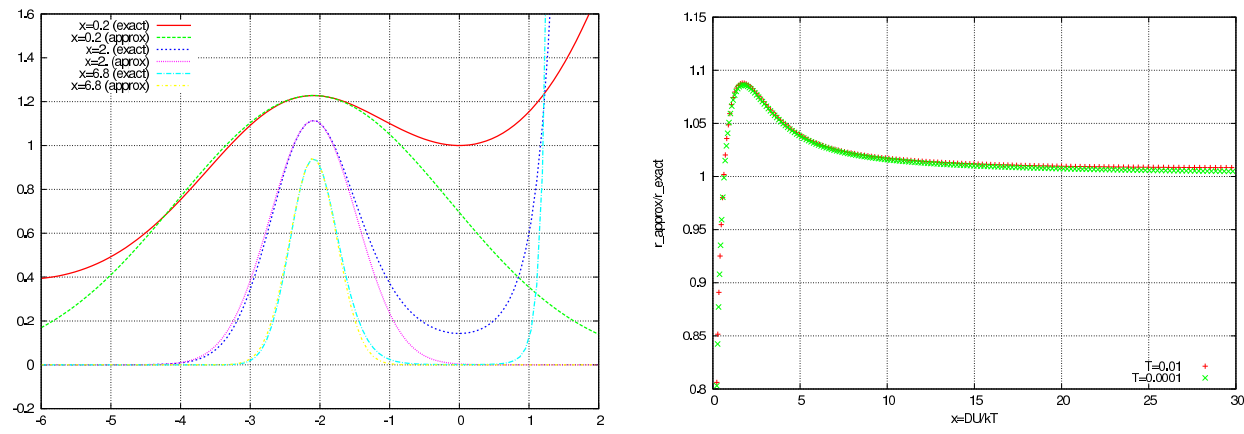

Figura 6.4: Izquierda: dibujamos la función exp $-\beta V(x)$-normalizada- para el potencial exacto y la aproximación parabólica al mismo $\left(r_{T S T}\right.$ se calcula integrando esta función). Usamos $V_{0}=10, F=V_{0} / 2$ y $\beta=0.03,0.03,1$; entonces $x=\Delta U / k_{B} T=0.2$, 2., 6.8 respectivamente. Derecha: $r_{T S T}^{\text {exact }} / r_{T S T}^{\text {approx }}$ como función de $\Delta U / k_{B} T$ para dos valores de $T$. Vemos que el resultado es el mismo para los dos $T$. La figura de la izquierda permite entender la de la derecha para cualquier barrera: ver que la expresión exacta integra de $-\infty$ a cero y la aproximación de $-\infty \mathrm{a}+\infty$.), así que el cociente es cercano a uno para $x$ grande, mayor que uno para $x$ pequeño y menor que uno para valores de $x$ muy pequeños.

\subsection{Tasa de escape: resultados numéricos}

Nuestro objetivo es utilizar detalladas simulaciones de dinámica molecular de la dinámica a bajo amortiguamiento para confrontar los resultados teóricos disponibles. A este respecto, debemos señalar que, debido al gran tiempo de computación necesarios para obtener resultados cuando el amortiguamiento es pequeño (los tiempos de escape son proporcionales al inverso del amortiguamiento) no hemos encontrado en la literatura resultados concluyentes sobre el problema enunciado.

En particular, vamos a mostrar que los pequeños efecto de barrera finita son muy importantes en el caso de amortiguamiento bajo, la convergencia con el resultado de barrera infinita es muy lento, y la teoría DH reproduce los resultados numéricos a cualquier barrera y amortiguamiento.

Para ello hemos integrado numéricamente la ecuación de Langevin (6.1) del sistema para diferentes valores de amortiguamiento y altura de la barrera. En nuestras simulaciones hemos calculado el tiempo medio para que el sistema alcance por vez primera la barrera de potencial, MFPT (mean-first-passagetime). Para valores bajos del amortiguamiento, tal tiempo medio corresponde a la inversa de la tasa de escape. De acuerdo con los marcos teóricos, las simulaciones se inician con las partículas situadas en el pozo de potencial metaestable y velocidad cero. Más adelante discutiremos algunas cuestiones fundamentales sobre el problema de las condiciones iniciales. Los valore medio mostrados han 

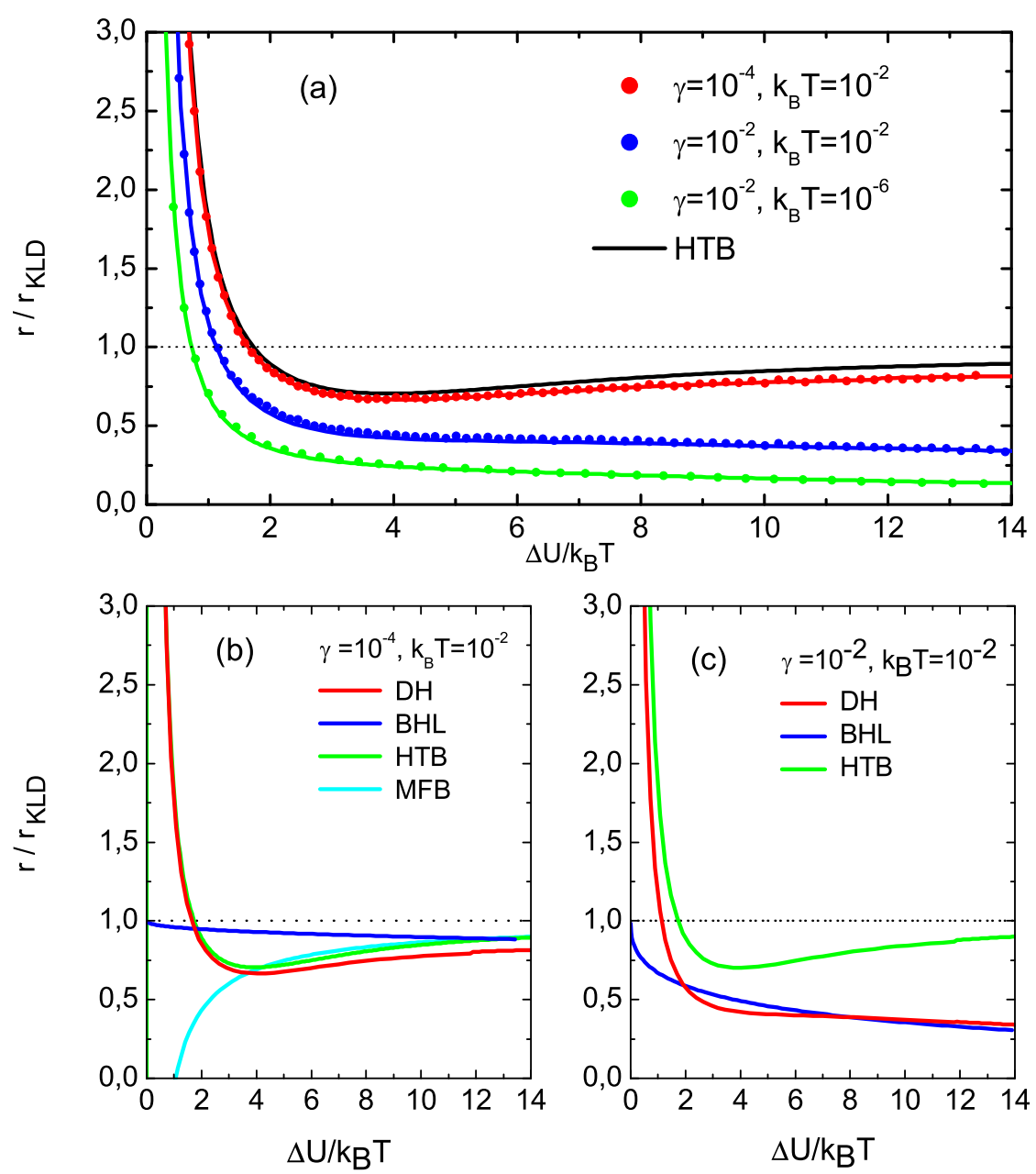

Figura 6.5: Tasa de escape dividida por el resultado de Kramers a bajo amortiguamiento, ecuación (6.7), como función de $\Delta U / k_{\mathrm{B}} T$. Figura (a): Los puntos son los resultados numéricos y las líneas en el color correspondiente la predicción teórica de DH. La línea negra representa la fórmula exacta de amortiguamiento tendiendo a cero, ecuación (6.19). La figura (b) y (c) compara los diferentes resultados teóricos.

sido calculados a partir $10^{4}$ eventos de escape. Mostramos los resultados para $V_{0}=0.155, m=0.35$ y diferentes valores de $I$, amortiguamiento y temperatura.

Los principales resultados están resumidos en las figuras 6.5 and 6.6 donde se muestra la tasa de activación en función de la barrera y el amortiguamiento, respectivamente, y se compara con algunas de las teorías existentes.

La figura 6.5 muestra la dependencia de la tasa de escape para diferentes valores de amortiguamiento y temperatura. Con el objeto de poder observar las 
diferencias entre los distintos resultados, dividimos las tasas obtenidas por el resultado de Kramers para amortiguamiento débil (6.7). Recordamos que (6.7) se obtiene suponiendo amortiguamiento débil y barrera alta. Por comparación, también dibujamos el resultado exacto para la barrera arbitraria en el límite de amortiguamiento tendiendo a cero [28], $r(\gamma \rightarrow 0)=r_{H T B}$. Podemos ver que las simulaciones realizadas a amortiguamiento $\gamma=10^{-4}$, muestran un resultado muy cercano al exacto, $r_{H T B}$. El amortiguamiento es muy pequeño pero a valores tan bajos de $\gamma$ aún se aprecian diferencias respecto el resultado el límite $\gamma \rightarrow 0$. En cualquier caso se aprecia una diferencia muy significativa con respecto el resultado de Kramers. Esta diferencia muestra que los efectos de tamaño finito son muy importantes en el régimen de amortiguamiento débil y prevalecen a valores de la barrera relativamente grandes como $\Delta U / k_{B} T=$ 10. El acercamiento al límite de barrera infinita es muy lento. Por ejemplo, $r_{H T B} / r_{K L D}=0.72$ para $\Delta U / k_{B} T=5 \mathrm{y} r_{H T B} / r_{K L D}=0.85$ para $\Delta U / k_{B} T=10$.

En las páginas anteriores, hemos descrito los dos principales intentos de incluir los efectos de barrera finita más allá del límite de amortiguamiento nulo. El primero de ellos es el de Melnikov [108]. Sin embargo, este resultado, como puede apreciarse en las figura 6.5(b) falla a barreras pequeñas aunque funciona razonablemente bien a barreras moderadas.

El segundo método fue propuesto por Drozdov y Hayashi [110] y como hemos visto, por construcción, recupera el resultado correcto en el límite $\gamma \rightarrow 0$ para barrera arbitraria. En nuestras simulaciones estudiamos la validez del resultado de DH más allá de este límite. Como puede verse en Las figuras. 6.5 and 6.6, la teoría DH explica nuestros resultados numéricos en toda la región de amortiguamiento débil-moderado.

En las figuras 6.5 (b) y (c) también mostramos resultados de la teoría BHL para $\alpha=1$. Vemos que la teoría falla para $\gamma=10^{-4}$ pero funciona apreciablemente bien si $\gamma=10^{-2}$ y $\Delta U / k_{B} T>6$. Las otras teorías que extienden el resultado de Kramers de bajo amortiguamiento al régimen de amortiguamiento moderado a bajo (MM, PGH), dan resultados bastante similares a BHL.

La figura 6.6 muestra la dependencia de la tasa de escape dividida por el amortiguamiento, $r / \gamma$, para $\Delta U / k_{B} T=3$ (figura superior) y $\Delta U / k_{B} T=10$ figura inferior, para dos valores de la temperatura y en función del amortiguamiento del sistema. Además de la concordancia con la teoría DH, comprobamos la convergencia de los resultados al límite $\gamma \rightarrow 0$ dado por $r_{H T B}$ (línea horizontal en ambas figuras). En el interior se dibujan los resultados de la teoría BHL. La figura permite cuantificar el rango de validez de cada uno de los resultados y en particular determinar el valor del amortiguamiento para el cual se entra en el rango de amortiguamiento muy pequeño. Además vemos que las correcciones de barrera finita se hacen menos importantes al aumentar el 

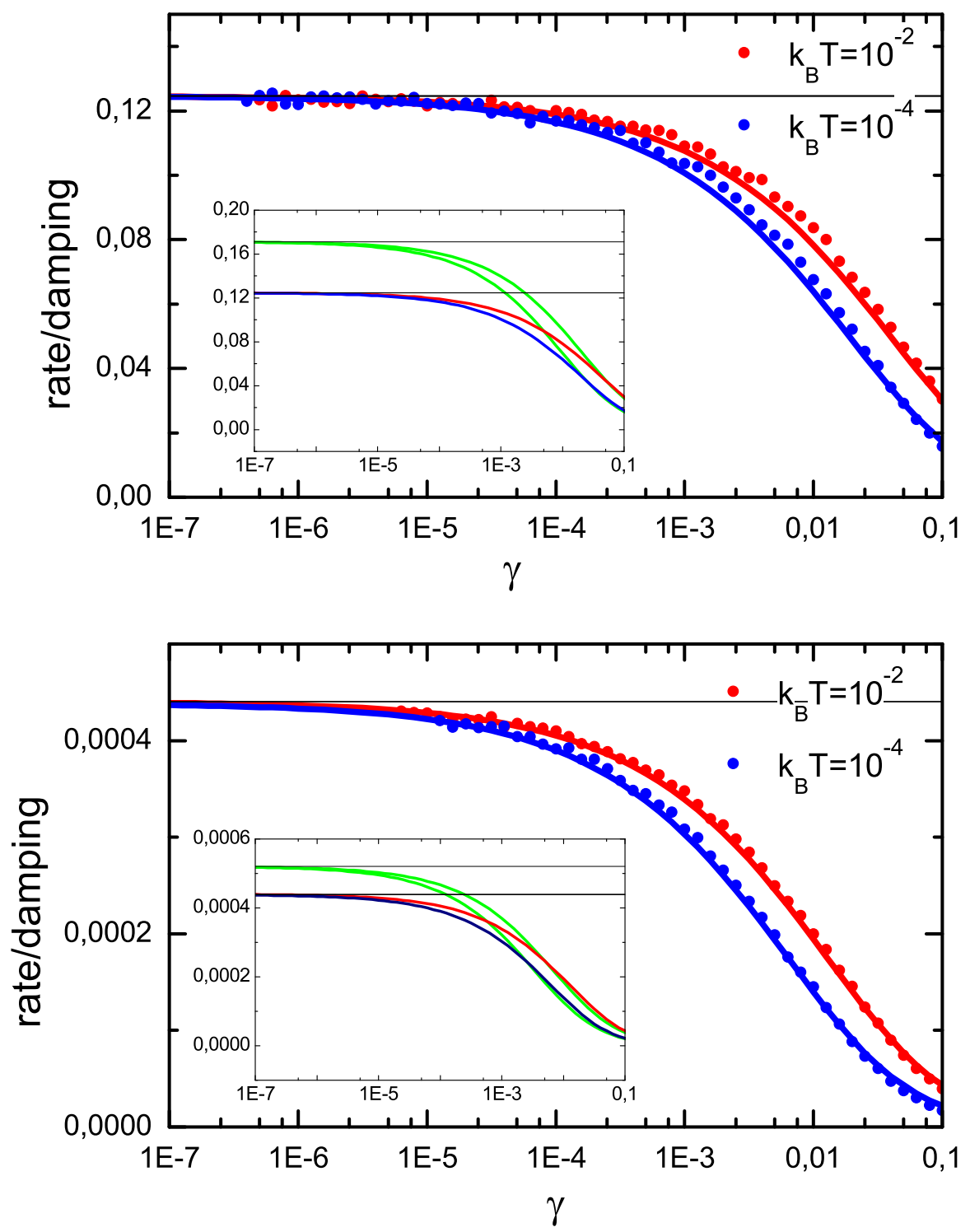

Figura 6.6: Dependencia de la tasa de escape con el amortiguamiento del sistema. Dibujamos $r / \gamma$. Los puntos son los resultados numéricos y las correspondientes líneas sólidas representan la teoría de DH. La línea negra horizontal es el límite de la ecuación con amortiguamiento tendiente a cero (6.19). Arriba: curvas de $\Delta U / k_{\mathrm{B}} T=3$. Abajo: $\Delta U / k_{\mathrm{B}} T=10$. Las figuras interiores comparan los resultados de $\mathrm{DH}$ con la teoría BHL (líneas verdes).

amortiguamiento, dado que el resultado BHL, que es un resultado de barrera infinita, se aproxima al valor exacto para $\gamma \sim 10^{-2}$.

Para completar nuestro análisis nos damos cuenta que al disminuir el amortiguamiento las curvas a diferentes temperaturas tienden a lo mismo, es decir, 
la tasa sólo depende de la relación $\Delta U / k_{\mathrm{B}} T$. Esto se entiende al notar que la mayor parte de la contribución en las integrales en la ecuación (6.19) provienen del fondo del potencial. Si la acción dentro del pozo se aproxima por la correspondiente a un potencial armónico con la misma frecuencia en el fondo, $E=\left(\omega_{0} / 2 \pi\right) J$, with $\omega_{0}=\partial_{x}^{2} V\left(x_{\min }\right)$, la tasa (6.19) se puede escribir como,

$$
r_{H A}=\gamma\left[\int_{0}^{\Delta U / k_{\mathrm{B}} T} \mathrm{~d} x \mathrm{e}^{-x} \int_{x}^{\Delta U / k_{\mathrm{B}} T} \mathrm{~d} y \frac{\mathrm{e}^{y}}{y}\right]^{-1},
$$

que claramente depende únicamente del cociente $\Delta U / k_{\mathrm{B}} T$. Resaltamos que, además de su simplicidad, la ecuación anterior es una excelente aproximación a (6.19). De hecho, si se dibuja el resultado (6.33) y el (6.19) en la figura 6.5, no es posible distinguir ambas curvas.

\subsection{El problema de las condiciones iniciales}

Nos planteamos ahora el estudio de la influencia de la condición inicial para la energía sobre los resultados de la tasa de escape. El escape térmico a bajo amortiguamiento es un problema de difusión de la energía. El escape se produce tan pronto como las fluctuaciones térmicas proporcionan suficiente energía a la partícula para superar la barrera. Si el amortiguamiento es pequeño, a determinados valores de la barrera este tiempo depende del valor inicial de la energía de la partícula.

Hasta ahora, para comparar nuestras simulaciones con la teoría, hemos supuesto que las partículas comienzan en el fondo del pozo metaestable con velocidad nula. Desde el punto de vista experimental esta suposición puede fallar. Las fluctuaciones térmicas no sólo proporcionan energía suficiente para superar la barrera sino también energía cinética en el fondo del pozo. Con el fin de estudiar la importancia de este tema hemos calculado las tasas de escape para dos nuevas condiciones iniciales diferentes, $v= \pm \sqrt{k_{\mathrm{B}} T / m}$ y las comparamos con una de velocidad inicial cero. Los resultados se muestran en la figura 6.7. Como era de esperar, se observa que para barreras pequeñas la presencia de una energía cinética inicial disminuye el tiempo de escape y por lo tanto incrementa la tasa de escape. La figura también muestra que para ciertos valores de $\Delta U / k_{\mathrm{B}} T$ los resultados son independientes de las condiciones iniciales utilizadas.

Para entender los resultados obtenidos usamos el siguiente razonamiento: Hemos visto que cuando las partículas se colocan con velocidad cero en la parte inferior del pozo, el tiempo de activación es $r^{-1}$. Sin embargo, si las partículas tienen energía inicial extra $E_{i n}$, el tiempo de escape será menor y vendrá 


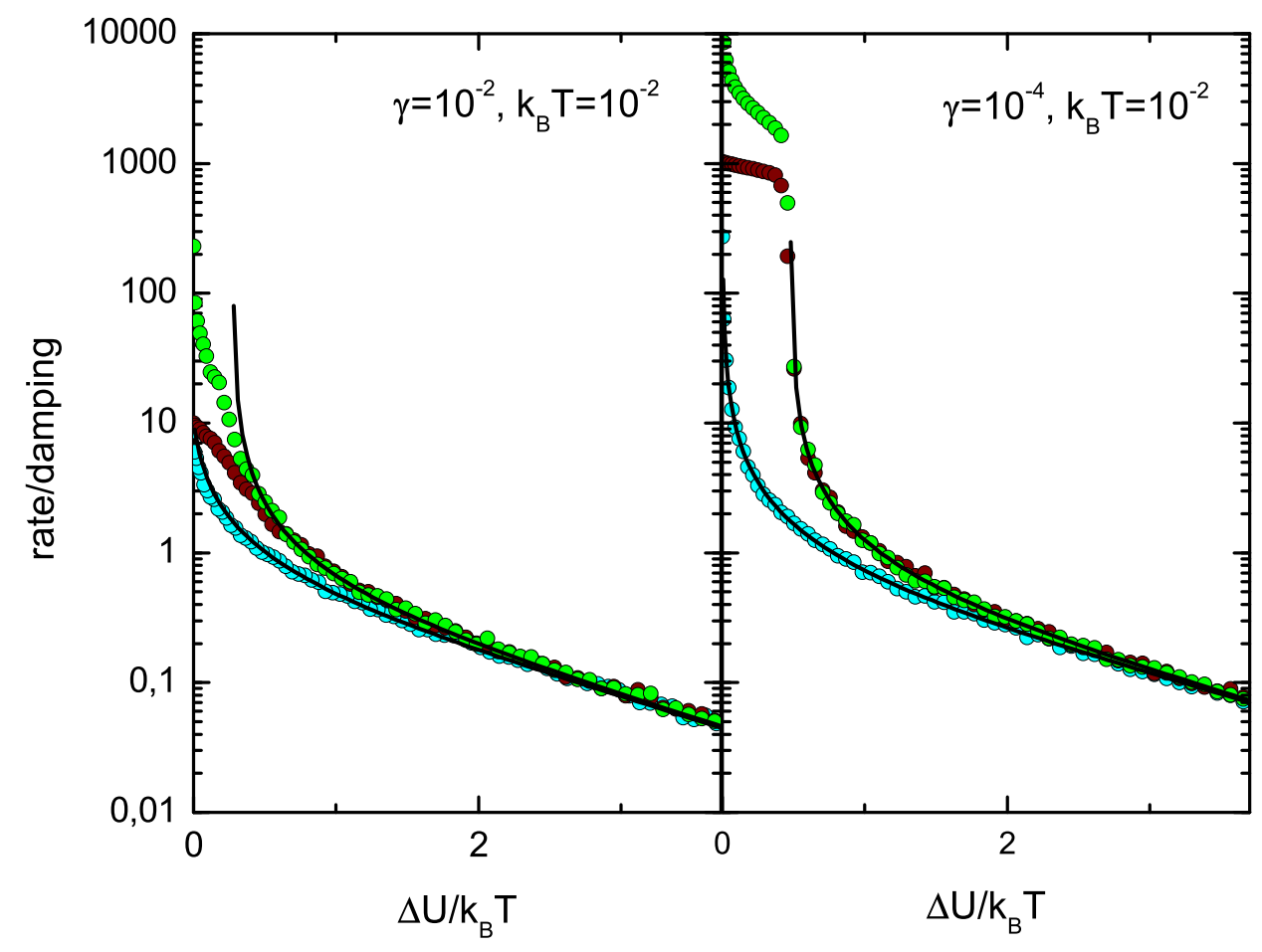

Figura 6.7: Tasa de activación vs barrera para tres condiciones iniciales: Las partículas están en el fondo del pozo metastable con velocidad cero (puntos azules), $v=\sqrt{k_{\mathrm{B}} T / m}$ (puntos verdes) o $v=-\sqrt{k_{\mathrm{B}} T / m}$ (puntos marrones). Las líneas representan las predicciones teóricas de la ecuación (6.34) para los casos con $E_{i n}=0.5 k_{B} T$.

dado por $r^{-1}-\tau$ donde $\tau\left(E_{i n}\right)$ da cuenta del tiempo de activación hasta esta energía extra, el cual se puede calcular en el límite de bajo amortiguamiento usando la ecuación (6.33) reemplazando $\Delta U$ by $E_{\text {in }}$ en ella. Juntando ambas consideraciones, podemos generalizar las fórmulas de la tasa de escape:

$$
r_{i n}=\frac{1}{r^{-1}-\tau}
$$

Esta ecuación muestra que tan pronto como $\tau\left(E_{\text {in }}\right) \sim r^{-1}$ el problema de las condiciones iniciales afecta las tasas de escape. En La figura 6.7, donde $E_{\text {in }}=k_{\mathrm{B}} T / 2$, esta corrección se vuelve importante para $\Delta U / k_{\mathrm{B}} T \lesssim 2$. Si $\Delta U \leq E_{\text {in }}$ el tiempo de paso es casi un proceso determinista que depende de la posición inicial y la velocidad del sistema. La figura 6.7 ilustra este efecto y confirma nuestra predicción teórica. 


\subsection{Resultados sobre la corriente de switching}

En un típico experimento de JJ la función de distribución de probabilidad de la corriente de switching de la unión $P(I)$ se mide realizando muchas curvas corriente-voltaje donde la corriente se incrementa constantemente a una velocidad dada. A partir de estos resultados la corriente de switching media $I_{\mathrm{sw}}$ y su desviación estándar se pueden calcular trivialmente. Tal $P(I)$ puede ser fácilmente calculada a partir de las tasas de escape $r(I)$ como [25]

$$
P(I)=r(I)\left(\frac{d I}{d t}\right)^{-1}\left(1-\int_{0}^{I} P(u) d u\right) .
$$

Invirtiendo esta relación,. también las tasas de escape pueden calcularse a partir de las medidas de $P(I)$.

En la figura 6.8(a) mostramos los resultados numéricos para el cálculo de la corriente de switching media a diferentes valores del amortiguamiento y los comparamos con las predicciones teóricas. Los resultados numéricos han sido obtenidos integrando la ecuación. (6.1) para un conjunto de uniones termalizadas a $I=0$. A partir de entonces la corriente externa se incrementa con una rampa dada y se registran los eventos de switching. Como es de esperar las predicciones basadas en BHL fallan en el régimen de amortiguamiento muy bajo. Sin embargo, sorprendentemente, también DH es incapaz de explicar nuestros resultados numéricos, que se encuentran en medio de ambas teorías. Esto se debe a la competencia entre el tiempo de equilibrio del sistema, dado por $\gamma^{-1}$, y el tiempo para el cambio de corriente, dado por la inversa de la rampa de corriente. Así, el switching en el régimen de muy bajo amortiguamiento es un proceso de no equilibrio. El acoplamiento al baño externo es tan débil que el conjunto de uniones no son capaces de alcanzar la energía de equilibrio térmica antes del switching. Así, el escape de las uniones se produce de manera similar a lo que se llama evaporative cooling, donde las uniones más energéticas son las primeras en saltar, y el conjunto de uniones que quedan atrapadas tienen una energía media inferior a la de equilibrio, están de modo efectivo enfriadas. Esta imagen se confirma en la figura. 6.8(c) donde, para un valor determinado del amortiguamiento, $\gamma=10^{-5}$ se muestra la energía media para las uniones atrapados en función de la corriente y la fracción de partículas que han escapado. Se puede observar como el conjunto inicialmente está termalizado, cociente 1, pero conforme comienzan los eventos de switching (contabilizados por la curva verde), la energía media de las uniones atrapadas disminuye.

También vemos en la figura 6.8(c) que el escape de partículas con un energía inicial que va desde $E_{\text {in }}=K_{\mathrm{B}} T$ a cero cuando se aumenta la corriente. La forma más sencilla de introducir este hecho en la teoría es asumir un valor promedio de $E_{\text {in }}=K_{\mathrm{B}} T / 2$ y utilizar nuestra ecuación (6.34). La figura 6.8(a) muestra 

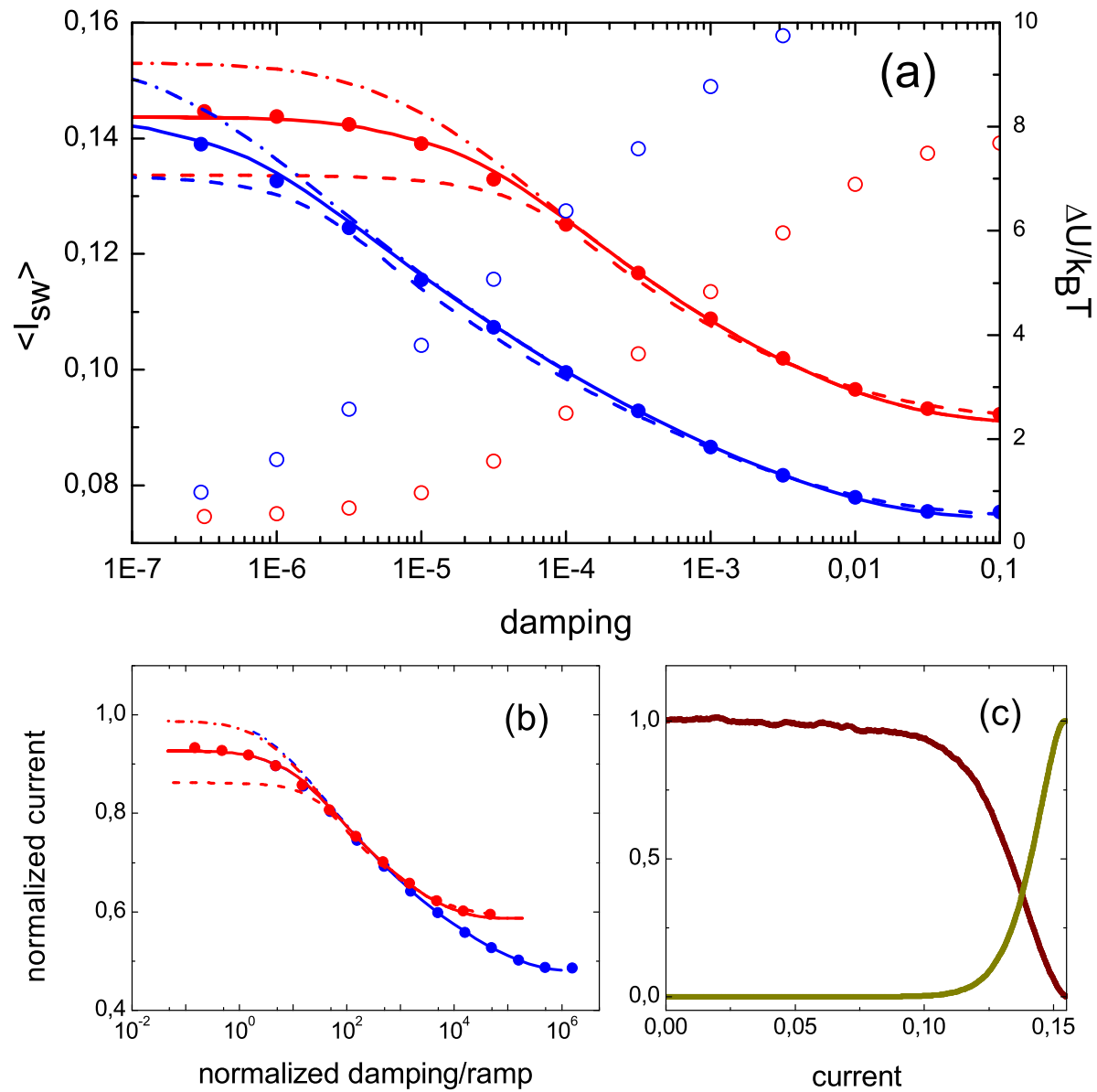

Figura 6.8: (a) Corriente de switching media a diferentes valores del amortiguamiento para $T=0.01$ y rampa de corriente $\dot{I}=3.33 \times 10^{-7}$ (rojo) y $\dot{I}=10^{-8}$ (azul). Los símbolos sólidos son para las simulaciones numéricas, las líneas a trazos para las predicciones basadas en la teoría BHL, las líneas punteadas para la teoría DH y las líneas sólidas para nuestra teoría, ecuación (6.34) con $E_{i n}=k_{B} T / 2$. También dibujamos (eje derecho) los valores de la barrera correspondiente a la corriente de switching media. (b) Dibujamos la corriente normalizada por 0.155 , su valor en el límite determinista, frente al amortiguamiento normalizado dividido por la rampa. (c) Energía media divida por $k_{B} T$ de las partículas en el pozo (marrón) y fracción de partículas que ha escapado (verde) como una función de la corriente aplicada $\left(T=0.01, \gamma=10^{-5}\right.$ y $\left.\dot{I}=3.33 \times 10^{-7}\right)$.

que de esta manera somos capaces de reproducir con bastante exactitud los resultados numéricos. Esta corrección resulta ser importante cuando la barrera media para la corriente de switching es del orden de la energía térmica. Véase que en la figura también se dibujó el valor de la barrera para la corriente 
de switching media (símbolos abiertos). Si el amortiguamiento es pequeño, el escape se produce en promedio para valores de $\Delta U / k_{B} T$ también pequeños. En ese caso los efectos de barrera finita y condiciones iniciales son especialmente importantes.

Finalmente, mediante las ecuaciones Eqs. (6.33), (6.34) and (6.35) se puede ver que en esta región del espacio de parámetros, los resultados dependen de la razón $\gamma / \dot{I}$, como se confirma en la figura 6.8(b). Por lo tanto nuestra teoría permite estimar los valores de $\gamma / \dot{I}$ donde son necesarias correcciones de noequilibrio. En la figura hemos usado parámetros normalizados. Para normalizar la ecuación (6.1) dividimos por $V_{0}$ y el tiempo por $\omega^{-1}=\sqrt{m / V_{0}}$. Entonces, los parámetros adimensionales son $\tilde{\gamma}=\gamma / \omega=1.50 \gamma, \tilde{T}=k_{B} T / V_{0}=6.45 k_{B} T$ y $\widetilde{I}=\dot{I} /\left(\omega V_{0}\right)=9.69 \dot{I}$ (recordamos que en nuestras simulaciones $V=0.155 \mathrm{y}$ $m=0.35)$. De este modo, la figura 6.8(b) permite una estimación del valor necesario para el cociente entre el amortiguamiento normalizado y la rampa para que los efectos de no equilibrio presentes en el artículo puedan ser observados.

\subsection{Discusión}

Los resultados obtenidos han estado motivados por el estudio de la dinámica de redes de uniones Josephson. Intentando entender os resultados mostrados en la figura 4.12, hemos acabado estudiando el problema del escape térmico a valores pequeños del amortiguamiento. Obviamente, se trata de un problema mucho más general, que ha suscitado enorme interés a lo largo de los últimos 30 años y cuyos resultados se aplican en muchas áreas científicas, en particular en todos aquellos sistemas que están acoplados débilmente con el entorno [114, $115,116]$.

Así, aunque hemos presentado nuestros resultados en el marco de las medidas de la corriente de switching en JJ, los resultados tienen una validez general y pueden aplicarse a cualquier experimento en el que se mide una tasa de activación en función de una parámetro externo que puede ser controlada a voluntad.

En particular, los resultados obtenidos conducen de manera natural al estudio del comportamiento de sistemas en el límite de amortiguamiento grande. Es interesante conocer los efectos de barrera finita en ese límite y los efectos de competencia entre escalas temporales (la natural del proceso de activación y la fijada por la velocidad de cambio de un parámetro externo de control) en los distintos procesos de activación térmica. De este modo buscaríamos transferir los resultados obtenidos en el régimen de amortiguamiento débil, energy-diffusion regime, al de amortiguamiento fuerte, phase-diffusion regime. 
Este es el caso típico de muchos de los experimentos actuales sobre fricción atómica $[117,118,119]$ o sobre la física de sistemas biológicos [120, 121]. 


\section{Discusión y conclusiones}

Motivados por trabajos experimentales recientes, en esta tesis doctoral se ha abordado el estudio detallado de las principales propiedades de fluxones atrapados en redes de uniones Josephson regulares y asimétricas. Nos hemos centrado fundamentalmente en el estudio de las curvas $I$ - $V$ del sistema en presencia de fluctuaciones térmicas y en la identificación y caracterización de los principales regímenes dinámicos presentes y las transiciones entre los mismos. En particular, se ha prestado una atención especial al problema del desanclaje térmico de fluxones en anillos regulares y ratchet de uniones Josephson y la comparación con los resultados basados en modelos monoparticulares. Así mismo, los resultados obtenidos en el régimen de amortiguamiento pequeño nos han llevado a estudiar el problema del escape térmico de una partícula en ese régimen dinámico. Por último, se ha identificado y estudiado por vez primera un estado de bajo voltaje de difusión del fluxón, excitado térmicamente, en redes subamortiguadas.

El sistema físico de interés es una red de uniones Josephson en paralelo dispuestas en geometría de anillo. Los métodos de fabricación de anillos superconductores con uniones Josephson permiten variar a voluntad el área de las uniones empleadas y el tamaño de las celdas entre uniones. Esta flexibilidad permite diseñar distintas configuraciones donde el potencial efectivo de los fluxones atrapados en la red sea el deseado. En particular destacamos el caso de redes regulares (uniones y celdas iguales), donde dicho potencial es simétrico; y el de redes ratchet (con alternancia de dos tipos de uniones y celdas distintas, o con celdas iguales y alternancia de uniones de tres tamaños diferentes), con potencial efectivo asimétrico. En el capítulo 3 hemos caracterizado las principales propiedades estáticas de fluxones en dichas configuraciones. Se han obtenido los potenciales de Peierls-Nabarro del sistema, evaluados las barreras de energía, corrientes de desanclaje, frecuencia y masas efectivas y validado algunas cuestiones de la imagen fluxón-partícula. Este conocimiento ha sido importante para lograr una mejor comprensión de los fenómenos que se han descrito en los capítulos 4,5 y 6 de la tesis. 
La curva característica de las redes de uniones Josephson es la llamada curva $I$ - $V$ del sistema que muestra el valor del voltaje medio en función de la corriente externa aplicada. En los capítulos 4 y 5 mostramos numerosos ejemplos de tales curvas calculados para distintos tipos de redes y un amplio abanico de parámetros de las mismas. En grandes rasgos estas curvas están caracterizadas por la presencia de una rama superconductora de voltaje nulo, una rama de bajo voltaje asociada al movimiento del fluxón en el anillo (está rama no se presenta si el amortiguamiento es muy pequeño) y una rama óhmica de alto voltaje. La transición entre las diferentes ramas ocurre a las llamadas corrientes de depinning y de switching del sistema y es discontinua en el caso de redes de amortiguamiento moderado o bajo y continua para redes sobreamortiguadas.

El primer objetivo de esta tesis doctoral es el estudio del depinning térmico de fluxones en anillos pequeños de uniones Josephson en el régimen de amortiguamiento débil. En este régimen, a temperatura cero, cuando la corriente se incrementa el sistema salta desde el estado de voltaje cero superconductor al estado resistivo de $v=i$. Dicho salto permite una clara caracterización de la corriente de depinning del sistema $i_{d e p}$. Si $i>i_{d e p}$ el sistema de ecuaciones del arreglo no admite soluciones estáticas, y el fluxón comienza a moverse. Debido al bajo valor del amortiguamiento, cuando el fluxón se mueve en el arreglo causa que todas las uniones cambien al estado de alto voltaje. Entonces todas las uniones rotan uniformemente pero con una diferencia de fase que tiene en cuenta la presencia de los cuanto de flujo homogéneamente distribuidos a lo largo del arreglo completo. Para amortiguamiento pequeño las curvas $I$ - $V$ del sistema presentan una histéresis importante. Al disminuir la corriente el sistema retorna al estado superconductor a valores de la corriente mucho menores que $i_{d e p}$. En nuestro trabajo nos hemos concentrado en el comportamiento del sistema al aumentar la corriente desde cero.

Debido a las fluctuaciones térmicas, en un experimento el valor de la corriente de desanclaje cambia desde una $I$ - $V$ a otra, es una variable aleatoria con una distribución de probabilidad determinada. Esta función está usualmente caracterizada por su valor medio y su desviación estándar. El principal objetivo de esta tesis fue estudiar numéricamente como se comportan estos observables para diferentes parámetros del sistema (acoplamiento $\lambda$, amortiguamiento $\Gamma$ y temperatura $T$ ) [123]. Dichos resultados se presentan en los capítulos 4 y 5 de la tesis.

Un punto importante del trabajo ha sido comparar nuestros resultados numéricos con los resultados basados en la imagen fluxón-partícula individual. Así, los resultados fueron estudiados en el marco teórico del escape térmico de una partícula en un potencial sinusoidal inclinado. La conclusión principal 
de nuestro trabajo es que para la mayoría de los casos esta imagen da una buena estimación de la corriente de desanclaje del fluxón. En efecto, en un caso experimental, donde los diferentes parámetros (principalmente $\lambda, \Gamma$ y $I_{c}$ ) son conocidos con alguna imprecisión será difícil identificar desviaciones del comportamiento esperado. Sin embargo, también hemos visto algunos efectos inesperados que los atribuimos a la discretitud. Así, para el caso de acoplamiento pequeño ( $\lambda=0.4$ y más pequeño) vemos un incremento en la desviación del comportamiento de desanclaje del fluxón desde lo que esperábamos para la imagen de una partícula. Los arreglos con acoplamientos mayores son más cercanos al límite del continuo y los efectos de discretitud son más pequeños; aquí la imagen de una partícula funciona mucho mejor.

Es evidente que nuestra aproximación no tiene en cuenta un número importante de aspectos difíciles de manejar: el potencial unidimensional efectivo para el fluxón en el arreglo (potencial de Peierls-Nabarro) no es puramente sinusoidal, y el valor de la masa del fluxón no es constante ya que depende de la posición del fluxón y el valor de la corriente. También estamos despreciando todos los grados de libertad del sistema excepto uno. Además hemos considerado la expresión para la tasa de escape en un caso unidimensional y el nuestro es multidimensional. Los primeros aspectos dan pequeñas correcciones a los resultados obtenidos y el caso mutidimensional usualmente se aborda con una redefinición de la frecuencia de intento que depende de la frecuencia de todos los modos estables en el mínimo y la silla [28]. Dicha frecuencia se calcula también en el capítulo 3 y se aproxima en gran medida a la frecuencia de Peierls-Nabarro del fluxón. En todo caso este concepto produciría cambios pequeños de los valores obtenidos con un error máximo menor del $7 \%$ (y ocurre para $\lambda=0,125)$. Por todo ello entendemos que es el papel relevante del resto de modos de libertad del sistema el que produce las discrepancias obtenidas y que este papel es importante para valores pequeños del acoplamiento $\lambda<0.4$ y despreciable por encima de este valor del mismo.

Nuestros resultados para una partícula están de acuerdo con las predicciones de la teoría de Kramers para la tasa de escape excepto para algunos límites. Se esperaba el desacuerdo a altas temperaturas ya que las expresiones teóricas están calculadas en el límite de barrera infinita del sistema $\left(E_{b} \gg k T\right)$. Este límite no se satisface a altas temperaturas. También es cierto a pequeñas temperaturas, donde la mayoría de eventos de escape ocurre a corrientes muy cercanas a $i_{\text {dep }}$ donde la barrera también es muy pequeña. Hemos observado que la razón $E_{b} / k T$ también en este caso es pequeña. Sin embargo fue una notable sorpresa identificar un importante desacuerdo entre los resultados de nuestras simulaciones y las predicciones teóricas para el escape de partículas en el régimen de amortiguamiento muy pequeño. Dicho hallazgo condujo el devenir del trabajo de tesis doctoral durante el último año y medio del mismo 
y es el origen de los resultados presentados en el capítulo 6 de la tesis y a los cuales nos referiremos más adelante.

Continuando con el estudio de la dinámica de fluxones, otro efecto inesperado observado a amortiguamiento pequeño es la emergencia de un estado de bajo voltaje excitado térmicamente, que llamamos difusión del fluxón. Para valores pequeños del amortiguamiento las curvas $I-V$ a temperatura cero no muestran ningún estado de bajo voltaje y el sistema salta desde voltaje cero al estado de alto voltaje. Sin embargo, a temperatura suficientemente alta se observa la aparición de una rama de bajo voltaje que hemos identificado con un movimiento de difusión térmica del fluxón. El voltaje de la red cambia primero desde cero a esta rama y a una corriente mayor al estado de alto voltaje. Una parte importante del capítulo 4 se ha dedicado a la caracterización de este movimiento de difusión del fluxón y se observa por ejemplo que el rango de valores de observación del mismo depende de manera importante del amortiguamiento y el tamaño de la red.

En la imagen fluxón-partícula, o el modelo RCSJ para una unión, generalmente se acepta que la difusión no puede coexistir con la histéresis. Kautz y Martinis [97] hacen alusión a esto a través de argumentos del espacio de fase: si el valor de la corriente aplicada es suficiente para permitir que el estado running estable coexista con los puntos fijos de voltaje cero, las cuencas de atracción para el estado running necesariamente se separan de las cuencas de atracción de cualquieras dos puntos fijos vecinos. De esta manera, los saltos de fase entre dos puntos fijos están prohibidos, ya que el sistema debe pasar primero a través de la cuenca de atracción del estado running. Mientras que en las secciones previas hemos mostrado que el escape inicial del fluxón desde su mínimo se puede explicar por activación térmica de una partícula, el estado de difusión del fluxón en las curvas $I$ - $V$ subamortiguadas no se puede explicar de manera similar.

En los experimentos sobre difusión de fase en uniones Josephson individuales con amortiguamiento débil, la coexistencia de la difusión de fase y la histéresis se explica por el efecto de la impedancia del circuito externo acoplado a la unión. Dicha impedancia introduce grados de libertad adicionales en el sistema y conduce a un amortiguamiento efectivo que depende de la frecuencia. En nuestro caso no existe tal circuito externo, pero el centro de masas del fluxón está acoplado al resto de grados de libertad del sistema total. Es la interacción entre el fluxón y el resto de modos del sistema, que han sido excitados térmicamente, lo que posibilita la existencia de un estado de difusión estable en el anillo a pesar del bajo valor del parámetro de amortiguamiento. El estudio de la estabilidad de la rama de difusión en redes de distinto tamaño a revelado que la estabilidad del mismo aumenta de manera importante con el 
tamaño del sistema.

La existencia de estados de bajo voltaje en el anillo permite la definición de una nueva corriente crítica, la corriente de switching que marca el salto a la rama óhmica de alto voltaje. El estudio del valor medio y la desviación estándar de la distribución de probabilidad asociada a esta corriente muestra que ambos parámetros dependen débilmente de la temperatura y la anchura de la distribución es pequeña en comparación con la anchura de las distribuciones para la corriente de depinning a temperaturas similares. Las curvas de la desviación estándar frente a la temperatura muestran un máximo a una temperatura dada que permite caracterizar la temperatura de aparición del estado de bajo voltaje en el sistema. Sin embargo, tal y como hemos mencionado anteriormente, esta corriente si que depende de manera importante del tamaño del sistema. El estudio de esta dependencia se está realizando en la actualidad.

Mencionaremos que hasta el desarrollo de esta tesis no existían resultados experimentales o numéricos que estudien sistemáticamente el escape térmico de fluxones o solitones en arreglos discretos. Guiados por nuestros resultados numéricos, en la actualidad el grupo del Dr. Kenneth Segall en la Colgate University está llevando a cabo una serie de experimentos al respecto. En ellos se ha confirmado con claridad la presencia de la rama de difusión de bajo voltaje descrita en esta memoria. Además, deseamos mencionar el trabajo hecho por A. Wallraff et al sobre vórtices en uniones Josephson largas [23], el análogo en el continuo de nuestro sistema. Con respecto a los avances teóricos queremos citar también el trabajo reciente [122] en donde se reporta la diferencia principal entre el efecto túnel cuántico macroscópico en uniones Josephson del efecto túnel de una partícula cuántica.

En el capítulo 5 hemos estudiado el caso de redes con potenciales asimétricos. En este caso se trata de redes de 9 uniones Josephson con tres tipos de corrientes críticas lo que al final, para valores del acoplamiento por encima de un umbral induce un potencial simétrico extendido a lo largo de tres celdas de la red y con un único mínimo y máximo por periodo. Los resultados muestran la asimetría en la definición de las corrientes de desanclaje y las curvas $I$ - $V$ del sistema. Comprobamos como las curvas obtenidas pueden entenderse en un marco común y son aproximadas razonablemente por la teoría de activación térmica de partículas. En cualquier caso se observa que las corrientes de escape de estas redes son mucho mayores que las corrientes críticas de redes regulares con valores de $\lambda$ similares. La diferencia entre la corriente de escape en las dos direcciones de la ratchet, para un valor específico del acoplamiento (de los simulados en este trabajo) se mantiene relativamente constante con la variación del amortiguamiento. Por otro lado, esta diferencia entre las corrientes de escape tiene un máximo para el amortiguamiento $\lambda=0.4$ comparado con los 
otros dos valores del acoplamiento, esto es, $\lambda=0.6$ y $\lambda=0.8$.

Es notable señalar que, en general, el sistema de nueva uniones con un fluxón en configuración ratchet, no presenta regiones de difusión, aún a temperaturas altas, hasta que el amortiguamiento es significativo. Pensamos que esto es debido al pequeño tamaño del sistema que consideramos (potencial periódico de tres pozos para el fluxón). Queda pendiente el estudio de este fenómeno en redes mayores.

Nuestro trabajo sobre el comportamiento de fluxones en redes asimétricas es un primer paso en un proyecto a largo plazo de diseño y medición de las propiedades de fluxones en ratchets cuánticas. Utilizando uniones mucho más pequeñas y temperaturas menores dicho estudio es en la actualidad factible desde un punto de vista experimental.

Como dijimos anteriormente, uno de los resultados más relevantes encontrados es la incapacidad de las predicciones teóricas usuales para dar cuenta de los resultados obtenidos a muy bajo amortiguamiento. Motivados por los resultados mostrados en las figuras 4.11 y 4.12 el capítulo 6 se dedica al estudio del problema de Kramers, el problema del escape térmico de un potencial metaestable, a valores del amortiguamiento muy pequeños. Nuestros resultados indican que en este régimen los efectos de barrera finita son fundamentales y la convergencia al resultado de barrera infinita muy lenta. Por ello las teorías habituales no son capaces de estimar con precisión las tasas de escape y en su lugar hay que emplear otros resultados. En nuestro trabajo demostramos que, entre las teorías existentes, la teoría de Drozdov y Hayashi, una combinación de los resultados exactos en el límite $\Gamma \rightarrow 0$ y el desarrollo de Melnikov que extiende los resultados de Kramers barrera infinita a valores moderados del amortiguamiento, es capaz de reproducir los resultados numéricos de las tasas de escape en todo el rango de barreras y amortiguamiento.

Sin embargo, los resultados sobre el valor medio de la corriente de escape aún no pueden ser entendidos satisfactoriamente, a pesar de emplear la teoría correcta para las tasas de escape. Hemos detectado que ello es debido a que para valores suficientemente pequeños del cociente entre el amortiguamiento y la rampa de la corriente el proceso de escape es un proceso de no equilibrio donde el escape "de las partículas más calientes del sistema" provoca que la energía promedio de las partículas atrapadas en el pozo sea menor que la energía térmica disponible. Basándonos en un estudio sobre el efecto de las condiciones iniciales en los procesos de escape a barrera pequeña hemos sido capaces de desarrollar una teoría sencilla que permite una correcta predicción teóricas de los resultados encontrados en las simulaciones. 


\section{Bibliografía}

[1] H. Kamerlingh Onnes, Leiden Comm. 120b, 122b, 124c (1911).

[2] J. Matricon and G. Waysand, The cold wars: an history of superconductivity, Rutgers University Press, 2003.

[3] T. P. Orlando and K. A. Delin, Foundations of applied superconductivity. Addison Wesley, 1991.

[4] M. Tinkham, Introduction to superconductivity. Mc Graw Hill, 1996.

[5] B. D. Josephson, "Possible new effect in superconductive tunneling". Phys. Lett. 1, 251-253 (1962).

[6] J. R. Waldram, "The Josephson effect in weakly coupled superconductors". Rep. Prog. Phys. 39, 751-821 (1976).

[7] A. Barone and G. Paternò, Physics and applications of the Josephson effect. John Wiley, 1982.

[8] K. K. Likharev, Dynamics of Josephson junctions and circuits. Gordon and Breach Science, 1984.

[9] T. Van Duzer and C. W. Turner, Principles of Superconductive Devices and Circuits. Pearson Education POD, 1998.

[10] J. Clarke, A. N. Cleland, M. H. Devoret, D. Esteve and J. M. Martinis, "Quantum mechanics of a macroscopic variable: the phase difference of a Josephson junction". Science 239, 992-997 (1988).

[11] J. Clarke, "Flux qubit completes the hat trick." Science 299, 1850 (2003).

[12] M. H. Devoret, A. Wallraff, and J. M. Martinis, "Superconducting qubits: a short review". arXiv:cond-mat/0411174 (2004).

[13] H. Mooij, "The road to quantum computing". Science 307, 1210 (2005). 
[14] J. Clarke and F. K. Wilhelm, "Superconducting quantum bits". Nature 453, 1031 (2008).

[15] M. Ansmann, H. Wang, R. C. Bialczak, M. Hofheinz, E Lucero, M. Neeley, A. D. O'Connell, D. Sank, M. Weides, J. Wenner, A. N. Cleland, and John M. Martinis, "Violation of Bell's inequality in Josephson phase qubits". Nature 461, 504 (2009).

[16] A. Barone, "Weakly coupled macroscopic quantum systems: likeness with difference". In Quantum Mesoscopic Phenomena and Mesoscopic Devices in Microelectronics, pp. 301-320. Edited by I. O. Kulik, Kluwer Academic, 2000.

[17] O. Avenel and E. Varoquax, "Josephson effect and quantum phase slippage in superfluids". Phys. Rev. Lett 60, 416-419 (1988).

[18] A. J. Legget "Bose-Einstein condensation in the alkali gases: some fundamental concepts". Rev. Mod. Phys. 73, 307-356 (2001).

[19] V. Ambegaokar and A. Baratoff, "Tunneling between superconductors". Phys. Rev. Lett. 10, 486-489 (1963) and erratum 11, 104 (1963).

[20] W. C. Stewart, "Current-voltage characteristics of Josephson junctions". Appl. Phys. Lett. 12, 277-280 (1968).

[21] D. E. McCumber, "Effect of ac impedance on dc voltage-current characteristics of superconductor weak-link junctions". J. Appl. Phys. 39, 3113-3118 (1968)

[22] A. V. Ustinov, "Solitons in Josephson junctions". Physica D 123, 315-329 (1998).

[23] A. Wallraff, A. Lukashenko, J. Lisenfeld, A. Kemp, M. V. Fistul, Y.Koval, and A. V. Ustinov, "Quantum dynamics of a single vortex". Nature 425, 155-158 (2003).

[24] J. Kurkijarvi, "Intrinsic Fluctuations in a Superconducting Ring Closed with a Josephson Junction". Phys. Rev. B 6, 832 (1972).

[25] T. A. Fulton and L. N. Dunkleberger "Lifetime of the zero-voltage state in Josephson tunnel junctions". Phys. Rev. B. 9, 4760 (1974).

[26] A. Garg, "Escape-field distribution for escape from a metastable potential well subject to a steadily increasing bias field". Phys Rev. B 51, 15592 (1995). 
[27] H. A. Kramers, "Brownian motion in a field of force and the diffusion model of chemical reactions". Physica (Utrecht) 7, 284-304 (1940).

[28] P. Hänggi, P. Talkner and M. Borkovec, "Reaction-rate theory: fifty years after Kramers". Rev. Mod. Phys. 62, 251-341 (1990).

[29] V. I. Mel'nikov, "The Kramers problem: fifty years of development". Phys. Rep. 209, 1-71 (1991).

[30] R. F. Voss and R. A. Webb, "Pair shot noise and zero-point Johnson noise in Josephson junctions". Phys. Rev. Lett. 47, 265 (1981)

[31] L. D. Jackel, J. P. Gordon, E. L. Hu, R. E. Howard, L. A. Fetter, D. M. Tennant, and R. W. Epworth, "Decay of the Zero-Voltage State in SmallArea, High-Current-Density Josephson Junctions". Phys. Rev. Lett. 47, 697 (1981).

[32] S. Washburn, R. A. Webb, R. F. Voss, and S. M. Faris, "Effects of Dissipation and Temperature on Macroscopic Quantum Tunneling". Phys. Rev. Lett. 54, 2712 (1985).

[33] M. H. Devoret, J. M. Martinis, and J. Clarke, "Measurements of Macroscopic Quantum Tunneling out of the Zero-Voltage State of a CurrentBiased Josephson Junction". Phys. Rev. Lett. 55, 1908 (1985).

[34] J. M. Martinis, M. H. Devoret, and J. Clarke, "Experimental tests for the quantum behavior of a macroscopic degree of freedom: The phase difference across a Josephson junction". Phys. Rev. B 35, 4682 (1987).

[35] P. Silvestrini, S. Pagano, R. Cristiano, O. Liengme, and K. E. Gray, "Effect of Dissipation on Thermal Activation in an Underdamped Josephson Junction: First Evidence of a Transition Between Different Damping Regimes". Phys. Rev. Lett 60, 1844 (1988).

[36] M. Büttiker, E. P. Harris and R. Landauer. "Thermal activation in extremely underdamped Josephson-junction circuits". Phys. Rev. B 28, 12681275 (1983).

[37] C. A. Hamilton, C. J Burroughs and S. P. Benz, "Josephson voltage standard: A review". IEEE Trans. Appl. Supercond. 7, 3756-3761 (1997).

[38] J. Clarke, "Principles and applications of SQUIDs". Proc. IEEE 77, 12081223 (1989).

[39] S. T. Ruggiero and D. A. Rudman (eds.), Superconducting Devices. Academic Press, 1990. 
[40] D. Koelle, R. Kleiner, F. Ludwig, E. Dantsker and J. Clarke, "Hightransition-temperature superconducting quantum interference devices". Rev. Mod. Phys. 71, 631-686 (1999).

[41] A. V. Ustinov, M. Cirillo and B. A. Malomed, "Fluxon dynamics in one-dimensional Josephson-junction arrays". Phys. Rev. B 47, 8357-8360 (1993).

[42] S. Watanabe, H. S. J. van der Zant, S. H. Strogatz and T. P. Orlando, "Dynamics of circular arrays of Josephson junctions and the discrete sineGordon equation". Physica D 97, 429-470 (1996).

[43] L. M. Floría and J. J. Mazo, "Dissipative dynamics of the FrenkelKontorova model". Adv. Phys. 45 505-598 (1996).

[44] O. Braun and Y.Kivshar, "Nonlinear dynamics of the Frenkel-Kontorova model". Phys. Rep. 306, 1-108 (1998).

[45] O. Braun and Y.Kivshar, The Frenkel-Kontorova model. Concepts, methods and applications. Springer, New York, 2004.

[46] E. Trías, J. J. Mazo and T. P. Orlando, "Discrete breathers in nonlinear lattices: experimental detection in a Josephson array". Phys. Rev. Lett. 84, 741-744 (2000).

[47] P. Binder, D. Abraimov, A. V. Ustinov, S. Flach, and Y. Zolotaryuk, "Observation of breathers in Josephson ladders". Phys. Rev. Lett. 84, 745-748 (2000).

[48] J. J. Mazo, "Discrete breathers in two-dimensional Josephson-junction arrays". Phys. Rev. Lett. 89, 234101-1(4) (2002).

[49] A. V. Ustinov, "Imaging of discrete breathers". Chaos, 13, 716-724 (2003).

[50] J. J. Mazo and T. P. Orlando, "Discrete breathers in Josephson arrays". Chaos, 13, 733-743 (2003).

[51] R. S. Newrock, C. J. Lobb, U. Geigenmüller and M. Octavio, "The twodimensional physics of Josephson-junction arrays". Sol. State Phys. 54, 263-512 (2000).

[52] P. Hadley, M. R. Beasley and K. Wiesenfeld, "Phase-locking of Josephsonjunction series arrays". Phys. Rev. B 38, 8712-8719 (1988).

[53] J. R. Friedman, V. Patel, W. Chen, S. K. Tolpygo and J. E. Lukens, "Quantum superposition of distinct macroscopic states". Nature 406, 43$46(2000)$ 
[54] L. Prandtl, Z. Angew. "Ein Gedankenmodell zur kinetischen Theorie der festen Körper". Math. Mech. 8, 85 (1928).

[55] U. Dehlinger, "Theory of recrystalization of pure metals". Ann. Phys. Lpz. 2, 749 (1929).

[56] Ya. Frenkel and T. Kontorova, Phys. Z. Sowietunion 13, 1 (1938).

[57] S. Aubry, "The twist map, the extended Frenkel-Kontorova model and the devil's staircase". Physica C 7, 240 (1983).

[58] S. Aubry, J.-P. Gosso, G. Abramovici, J.-L. Raimbault, and P. Quemerais, " Effective discommensurations in the incommensurate ground states of the extended Frenkel-Kontorowa models". Physica D 47, 461 (1991).

[59] See for instance the book by A. Scott, Nonlinear Science: Emergence 83 Dynamics of Coherent Structures. Oxford University Press (1999).

[60] M. Remoissenet, Waves called solitons: concepts and experiments, Springer 1999.

[61] T. Dauxois and M. Peyrard, Physics of solitons, Cambridge University Press 2006.

[62] Astumian, "Thermodynamics and kinetics of a Brownian motor". Science 276, 917-922 (1997).

[63] Astumian y Hänggi, "Brownian Motors". Physics Today 55(11) 33-39, (2002).

[64] P. Reimann, "Brownian motors: noisy transport far from equilibrium". Physics Reports 361, 57-265 (2002).

[65] P. Hänggi, F. Marchesoni, "Artificial Brownian motors: Controlling transport on the nanoscale". Rev. Mod. Phys. 81, 387 (2009)

[66] P. Nelson, Biological Physics: Energy, Information, Life. W. H. Freeman, New York, 2004.

[67] K. A. Dill, S. Bromberg Molecular Driving Forces: Statistical Thermodynamics in Chemistry and Biology Garland Science, New York, 2002.

[68] Hänggi, P., F. Marchesoni, and F. Nori, "Brownian motors". Ann. Phys. 14, 51-70, 2005

[69] Feynman, R. P., R. B. Leighton, and M. Sands, "The Feynman Lectures on Physics". Addison-Wesley, Reading, MA, Vol. I, 1963 
[70] Parrondo, J. M. R., and P. Espanol, "Criticism of Feynman's analysis of the ratchet as an engine". Am. J. Phys. 64, 1125-1130, 1996

[71] Schneider, W., and K. Seeger, "Harmonic mixing of microwaves by warm electrons in germanium". Appl. Phys. Lett. 8, 133-135, 1966.

[72] Marchesoni, F., "Harmonic mixing signal: Doubly dithered ring laser gyroscope". Phys. Lett. A 119, 221-224, 1986.

[73] Goychuk, I., and P. Hänggi, 1998, "Quantum rectifiers from harmonic mixing". Europhys. Lett. 43, 503-509, 1998.

[74] S. Savel'ev, F. Marchesoni, P. Hänggi, and F. Nori, "Transport via nonlinear signal mixing in ratchet devices". Phys. Rev. E 70, 066109, 2004a.

[75] S. Savel'ev, F. Marchesoni, P. Hänggi, and F. Nori, "Nonlinear signal mixing in a ratchet device". Europhys. Lett. 67, 179-185, 2004b.

[76] Olson, C. J. et. al. "Collective Interaction-Driven Ratchet for Transporting Flux Quanta". Phys. Rev. Lett. 87 177002, 2001

[77] J. E. Villegas,S. Savel'ev, F. Nori, E. M. Gonzalez, J. V. Anguita, R. García, and J. L. Vicent, "A superconducting reversible rectifier that controls the motion of magnetic flux quanta". Science 302(5648), 1188 1191 (2003).

[78] D.E. Shalom and H. Pastoriza, " Vortex Motion Rectification in Josephson Junction Arrays with a Ratchet Potential". Phys. Rev. Lett. 94, 177001 (2005).

[79] T. Guerin1, J. P. Pascal and J.F Joanny "Coordination and collective properties of molecular motors: theory". Current Opinion in Cell Biology, 22, 14-20, (2010)

[80] Magnasco M. O. "Forced Thermal Ratchet". Phys. Rev. Lett. 71, 1477, (1993).

[81] R. Bartussek, P. Hänggi, J. G. Kissner "Periodically Rocked Thermal Ratchets". Europhys. Lett., 28, 459-464, (1994)

[82] Siwy, Z.; Kosinska, I.D.; Fulinski, A.; Martin, C.R. "Asymmetric Diffusion through Synthetic Nanopores". Phys. Rev. Lett 94, 048102, 1-4 (2005).

[83] F. Falo, P. J. Martínez, J. J. Mazo and S. Cilla, "Ratchet potential for fluxons in Josephson-junction arrays". Europhys. Lett. 45, 700-706 (1999).

[84] E. Trías, J. J. Mazo, F. Falo and T. P. Orlando, "Depinning of kinks in a Josephson-junction ratchet array". Phys. Rev. E 61, 2257-2266 (2000). 
[85] F. Falo, P. J. Martínez, J. J. Mazo, T. P. Orlando, K. Segall and E. Trías, "Fluxon ratchet potentials in superconducting circuits". Appl. Phys. A 75, 263-269 (2002).

[86] P. J. Martínez et al., "Mode locking in discrete soliton dynamics under ac forces". Phys. Rev. B. 56, 87 (1997).

[87] C. Cattuto, G. Constantini, T. Guidi and F. Marchesoni. "Elastic strings in solids: Discrete kink diffusion". Phys. Rev. B 63094308 (2001).

[88] C. Cattuto, G. Constantini, T. Guidi and F. Marchesoni. "Driven kinks in discrete chains: Phonon damping". Phys. Rev. B 63046611 (2001).

[89] V. L. Pokrovsky. "Splitting of commensurate-incommensurate phasetransition". J. Phys. (Paris) 42, 761 (1981)

[90] K. Segall, private comunication (2007).

[91] H. S. J. van der Zant, T. P. Orlando, S. Watanabe, and S. H. Strogatz, "Kink Propagation in a Highly Discrete System: Observation of Phase Locking to Linear Waves". Phys. Rev. Lett. 74, 174 (1995).

[92] A. V. Ustinov, M. Cirillo, B. H. Larsen, V. A. Oboznov, P. Carelli, and G. Rotoli, "Experimental and numerical study of dynamic regimes in a discrete sine-Gordon lattice". Phys. Rev. B 51, 3081 (1995).

[93] S. Watanabe, S. H. Strogatz, H. S. J. van der Zant, and T. P. Orlando, "Whirling Modes and Parametric Instabilities in the Discrete SineGordon Equation: Experimental Tests in Josephson Rings". Phys. Rev. Lett. 74, 379 (1995).

[94] Z. Zheng, B. Hu, and G. Hu, "Resonant steps and spatiotemporal dynamics in the damped dc-driven Frenkel-Kontorova chain". Phys. Rev. B 58, 5453 (1998).

[95] J. Pfeiffer J, A. A. Abdumalikov, M. Schuster, A. V. Ustinov, "Resonances between fluxons and plasma waves in underdamped Josephson transmission lines of stripline geometry". Phys. Rev. B. 77024511 (2008).

[96] V. I. Mel'nikov and S. V. Meshkov, "Theory of activated rate processes: Exact solution of the Kramers problem". J. Chem.Phys. 851018 (1986).

[97] R. L. Kautz, and J. M. Martinis, "Noise-Affected I-V Curves in Small Hysteretic Josephson Junctions". Phys. Rev. B 42, 9903 (1990). 
[98] J. M. Kivioja, T. E. Nieminen, J. Claudon, O. Buisson, F. W. J. Hekking, and J. P. Pekola, "Observation of Transition From Escape Dynamics to Underdamped Phase Diffusion in a Josephson Junction". Phys. Rev. Lett. 94, 247002 (2005).

[99] V. M. Krasnov, T. Bauch, S. Intiso, E. Hürfeld, T. Akazaki, H. Takayanagi, and P. Delsing, "Collapse of thermal activation in moderately damped Josephson junctions". Phys. Rev. Lett. 95, 157002 (2005).

[100] J. Männik, S. Li, W. Qiu, W. Chen, V. Patel, S. Han, and J. E. Lukens, "Crossover from Kramers to phase-diffusion switching in moderately damped Josephson junctions". Phys. Rev. B. 71, 220509(R) (2005).

[101] J. Pfeiffer, M. Shuster, A.A. Abdumalikov, Jr., and A. V. Ustinov "Observation of Soliton Fusion in a Josephson Array". Phys. Rev. Lett. 96 034103 (2006).

[102] E. Pollak and P. Talkner, "Reaction rate theory: What it was, where is it today, and where is it going?" Chaos 15, 026116 (2005).

[103] V. Ambegaokar and B. I. Halperin, "Voltage Due to Thermal Noise in the dc Josephson Effect". Phys. Rev. Lett. 22, 1364 (1969).

[104] M. J. Stephen, "Noise in a Driven Josephson Oscillator". Phys. Rev. 186, 393 (1969).

[105] J. J. Mazo, F. Naranjo, and K. Segall, "Thermal depinning of fluxons in discrete Josephson rings". Phys. Rev. B 78, 174510 (2008).

[106] H. Grabert, "Escape from a Metastable Well: The Kramers Turnover Problem". Phys. Rev. Lett. 61, 1683 (1988).

[107] E. Pollak, H. Grabert, and P. Hänggi, "Theory of activated rate processes for arbitrary frequency dependent friction: Solution of the turnover problem". J. Chem. Phys. 91, 4073 (1989).

[108] V. I. Melnikov, "Activated Decay Rate: Finite Barrier Correction". Phys. Rev. E 48, 3271 (1993).

[109] R. Ferrando, R. Spadacini, G. E. Tommei, and V. I. Melnikov, "Numerical test of finite-barrier corrections for the hopping rate in the underdamped regime". Phys. Rev. E 51, 1645 (1995).

[110] A. N. Drozdov and S. Hayashi, "Self-similar renormalization approach to barrier crossing processes". Phys. Rev. E 60, 3804 (1999). 
[111] H. Risken and K. Voigtlaender, "Eigenvalues and Eigenfunetions of the Fokker-Planek Equation for the Extremely Underdamped Brownian Motion in a Double-Well Potential". J. Stat. Phys. 41, 825 (1985).

[112] S. Linkwitz, H. Grabert, E. Turlot, D. Steve and M. H. Devoret, "Escape Rates in the Region Between the Kramers Limits". Phys. Rev. A 45, R3369 (1992)

[113] B. Carmeli, and A. Nitzan, "Theory of Activated Rate Processes: Bridging between the Kramers Limits". Phys. Rev. Lett. 51, 233 (1983).

[114] D. J. McCarthy, y W. T. Coffey, "Kramers low-damping escape rate for fine ferromagnetic particles by the uniform asymptotic expansion of the first-passage-time method". J. Phys: Condens. Matter 11, 10531 (1999)

[115] J. L. García-Palacios, y F. J. Lázaro, "Kramers low-damping escape rate for fine ferromagnetic particles by the uniform asymptotic expansion of the first-passage-time method". Phys. Rev. B 58, 14937 (1998).

[116] J. A. Pelesko y D. H. Bernstein, Modeling MEMS and NEMS (Chapman and $\mathrm{Hall} / \mathrm{CRC}, 2003)$.

[117] Y. Sang, M. Dubé, y M. Grant, "Thermal Effects on Atomic Friction". Phys. Rev. Lett. 87, 174301 (2001)

[118] I. Barel, M. Urbakh, L. Jansen, and A. Schirmeisen, "Multibond Dynamics of Nanoscale Friction: The Role of Temperature". Phys. Rev. Lett. 104, $066104(2010)$

[119] O. K. Dudko, A. E. Filippov, J. Klafter, and M. Urbakh, "Beyond the conventional description of dynamic force spectroscopy of adhesion bonds". PNAS 100, 11378 (2003).

[120] C. Hyeon and D. Thirumalai, "Can energy landscape roughness of proteins and RNA be measured by using mechanical unfolding experiments?" PNAS 100, 10249 (2003);

[121] O. K. Dudko, "Single-molecule mechanics: New insights from the escapeover-a-barrier problem". PNAS 106, 8795 (2009).

[122] A. O. Sboychakov, S. Savel'ev, A. L. Rakhmanov, and Franco Nori, "Why macroscopic quantum tunnelling in Josephson junctions differs from tunnelling of a quantum particle". EPL 80, 17009 (2007).

[123] El valor también depende de la rampa de corriente la cual entra en las ecuaciones de un modo bien establecido por la teoría. 
